\title{
Towards Multiclass Damage Detection and Localization using Limited Vibration Measurements
}

Sandeep Sony

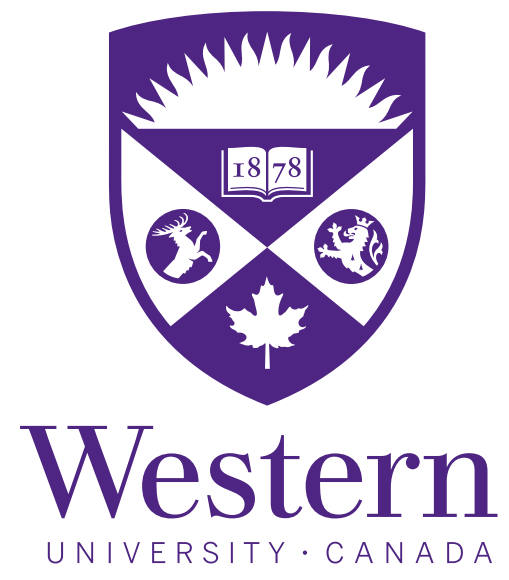

A thesis

presented to the University of Western Ontario in fulfillment of the

thesis requirement for the degree of

Doctor of Philosophy

in

Structural Engineering

(Specialization in Scientific Computing)

(Thesis Type: Integrated Article)

London, Ontario, Canada, 2021

(C) Sandeep Sony 2021 


\section{Author's Declaration}

I hereby declare that I am the sole author of this thesis. This is a true copy of the thesis, including any required final revisions, as accepted by my examiners.

I understand that my thesis may be made electronically available to the public. 


\begin{abstract}
Traditional vibration-based damage detection methods provide structural health information based on their measured data (i.e., acceleration and displacement response). Over the last few decades, various model-based and time-frequency methods have shown great promises for damage identification and localization. However, the existing methods are unable to perform satisfactorily in many situations, including the presence of limited sensor measurements and training data, detection of minor and progressive damage, and identification of multiclass damage, creating constraints to make them free of user-intervention and implemented using the modern sensors. The main objective of this thesis is to develop algorithms capable of damage identification and localization using limited measurements that can address the limitation of the traditional methods while providing a minimal to no user-intervention damage identification process.
\end{abstract}

The proposed research in this thesis involves casting damage detection problems as non-parametric and autonomous with the least user intervention. Progressive damage identification is presented using novel time-frequency methods, such as synchrosqueezing transform and multivariate empirical mode decomposition, showing improved sensitivity of identifying minor damage over traditional methods. A basis-free method, such as multivariate empirical mode decomposition, is employed for damage localization using limited sensors. The acquired vibration measurement is decomposed into its mono components, and a damage localization index based on modal energy is proposed to overcome the need for a large number of sensors. The limited measurement aspect of damage localization is explored by selecting fewer sensors, and it is shown that with limited measurements, the 
proposed method is as effective as a total number of measurements equals the number of degrees of freedom of the model.

To create an autonomous damage identification framework, Artificial Intelligence-based methods are explored the first time for multiclass damage classification and localization. Due to the lack of availability of a large amount of data, the acquired vibration data is augmented using windowing of the data per damage class. A novel window-based onedimensional convolutional neural network is explored to classify sequential time-series of vibration measurements with only one hidden layer. The robustness of the proposed method is further evaluated by a suite of parametric and sensitivity analysis. Improvement of this method is further accomplished by implementing a windowed Long Short-term Memory network capable of learning long-term dependencies of the sequential data. Finally, the proposed methods are validated using a suite of experimental and full-scale studies, including a high-rate dynamics experimental testbed, a stadia prototype experimental setup, the MIT green building, and the Z24 bridge. 


\section{Summary for Lay Audience}

Large-scale civil structures, such as buildings, bridges, stadiums, or roads, degrade with time due to various operational, environmental and human-made factors. To effectively utilize the build infrastructure during their intended design life, it is crucial to monitor them in a timely manner and provide any necessary maintenance required for their efficient performance to our citizens. The proposed research of this $\mathrm{PhD}$ thesis is focused on exploring cost-effective strategies for structural monitoring and identifying any defects in the structures using a fewer number of sensors. It is emphasized that the proposed strategies are user-intervention free and capable of creating an autonomous monitoring framework. The critical component of this research is to utilize limited sensors to reduce the financial burden on structural health monitoring communities and infrastructure owners. Advanced pattern recognition methods capable of providing information about both the time and instance of damage, and innovative artificial intelligence algorithms are evaluated for effective damage identification and localization in various types of structures using limited sensors and condition data. Through the proposed research, an autonomous infrastructure monitoring framework is developed for the health monitoring of structures subjected to a wide range of damage. 


\section{Dedication}

This thesis is dedicated to my family, and my late maternal grandparents, 'Shinda and Pyaro'. 


\section{Table of Contents}

List of Figures $\quad$ xi

List of Tables $\quad$ xvi

1 Introduction $\quad 1$

1.1 General Introduction . . . . . . . . . . . . . . . . . . . . 1

1.2 Scope of the Thesis . . . . . . . . . . . . . . . . . 3

1.3 General Objectives . . . . . . . . . . . . . . . . . 4

1.4 Organization of the Thesis . . . . . . . . . . . . . . 5

$\begin{array}{lll}2 & \text { Literature Review } & 7\end{array}$

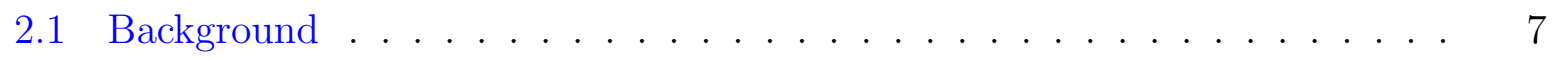

2.2 Traditional VDD Methods . . . . . . . . . . . . . . . . . 12

2.2.1 Time-frequency methods . . . . . . . . . . . . . . . . 12

2.2.2 Pattern recognition methods . . . . . . . . . . . . . . . 14

2.3 Drawbacks of the Traditional VDD Methods . . . . . . . . . . . . . . 24

2.4 Modern Techniques in VDD . . . . . . . . . . . . . . . . 25

2.4.1 Advanced TF methods . . . . . . . . . . . . . . . . 26

2.4.2 Deep Learning methods . . . . . . . . . . . . . . . . . . . . . 29

2.5 Challenges in the Modern VDD Methods . . . . . . . . . . . . . . . 32

2.6 Thesis Objectives . . . . . . . . . . . . . . . . . . . 34

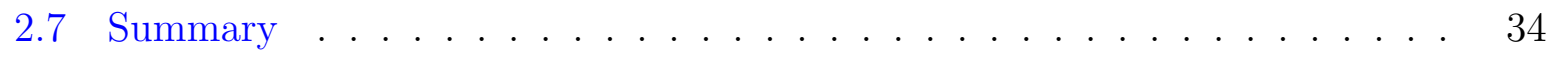


3.1 Introduction . . . . . . . . . . . . . . . . . . . 37

3.2 Background ............................ 40

3.2.1 Synchro-squeezing Transform $(\mathrm{SST}) \ldots \ldots$. . . . . . . . . . . 40

3.2.2 Multivariate EMD . . . . . . . . . . . . . . . . . . 45

3.3 Proposed Algorithm . . . . . . . . . . . . . . . . . . 48

3.4 Numerical Validation . . . . . . . . . . . . . . . . . . 50

3.4.1 SDOF model . . . . . . . . . . . . . . . . . 50

$3.4 .2 \quad 4$-DOF model . . . . . . . . . . . . . . . . . 55

3.5 Experimental Validation . . . . . . . . . . . . . . . . . 59

3.6 Full-scale Validation . . . . . . . . . . . . . . . . . . . . . . . 62

3.6.1 Details of full-scale data . . . . . . . . . . . . . . . . 62

3.6 .2 Identification results . . . . . . . . . . . . . . 63

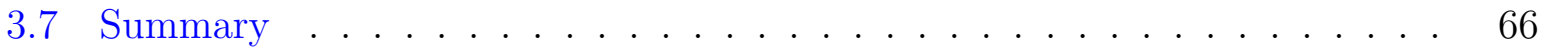

4 Damage Localization using MEMD-based Nonparametric Approach 67

4.1 Introduction . . . . . . . . . . . . . . . . . . 68

4.2 Proposed Algorithm . . . . . . . . . . . . . . . . 71

4.3 Numerical Studies . . . . . . . . . . . . . . . . . . . . . . . 73

4.3.1 Vibration data induced by Imperial Valley earthquake . . . . . . . 75

4.3.2 Vibration data induced by Borrego Mountain earthquake . . . . . . 81

4.4 Full-scale Study . . . . . . . . . . . . . . . . . . . . . . . 83

4.4.1 Details of the full-scale study . . . . . . . . . . . . 83

4.4 .2 Identification results . . . . . . . . . . . . . 85

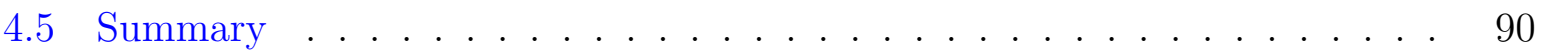

5 Multiclass Damage Identification using One Dimensional Convolutional $\begin{array}{ll}\text { Neural Networks } & 92\end{array}$ 
5.1 Introduction . . . . . . . . . . . . . . . . . . . . . . . . . . 93

5.2 Proposed Methodology . . . . . . . . . . . . . . . . . . . . . . . 97

5.2 .1 Background . . . . . . . . . . . . . . . . . . . 97

5.2.2 Multiclass damage detection using windowed-voted 1D CNN . . . 100

$5.2 .3 \quad$ Performance criteria . . . . . . . . . . . . . . . . . . . 102

5.2 .4 Damage localization . . . . . . . . . . . . . . . 105

5.3 Full-scale Study . . . . . . . . . . . . . . . . . . . . . . . . . 106

5.3.1 Details of Z24 Bridge . . . . . . . . . . . . . . . 106

5.3.2 Hyper-parameters of the 1D CNN model . . . . . . . . . . . 108

5.3.3 Random initialization of weights . . . . . . . . . . . . . . 109

5.3 .4 Effect of window size . . . . . . . . . . . . . . . 110

5.3.5 Model performance . . . . . . . . . . . . . . . . . . . . . . . 112

5.3 .6 Damage localization . . . . . . . . . . . . . . . . . 115

5.4 Summary . . . . . . . . . . . . . . . . . . . . 120

\section{Improved Multiclass Damage Localization using Windowed Long Short-} term Memory Networks 122

6.1 Introduction . . . . . . . . . . . . . . . . . . . . 123

6.2 Proposed Methodology . . . . . . . . . . . . . . . . . . . . . . . . 128

6.2 .1 Preliminaries of LSTM model . . . . . . . . . . . . . . . 128

6.2.2 Damage detection using the proposed LSTM model . . . . . . . . . 132

6.2.3 Damage localization using the proposed LSTM model . . . . . . . 136

6.2 .4 Performance criteria . . . . . . . . . . . . . . . 136

6.3 Performance Evaluation . . . . . . . . . . . . . . . . . . . . . . 139

6.3 .1 Experimental study . . . . . . . . . . . . . . . . . . . . . . . . 139

6.3 .2 Full-scale study . . . . . . . . . . . . . . . . . . . . . . . . 148

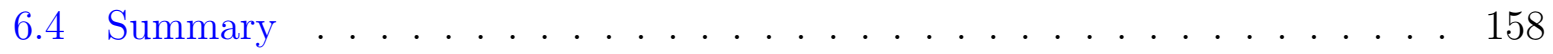


$\begin{array}{lll}7 & \text { Key Conclusions and Discussions } & 160\end{array}$

7.1 Key Conclusions . . . . . . . . . . . . . . . . . . . . . . 160

7.2 Thesis Contributions . . . . . . . . . . . . . . . . . . . . 162

7.2 .1 Journal papers . . . . . . . . . . . . . . . . . . . . 163

7.2 .2 Conference papers . . . . . . . . . . . . . . . . . . 164

7.3 Future Work . . . . . . . . . . . . . . . . . . . . . . . . . . . . 164

$\begin{array}{ll}\text { References } & 166\end{array}$ 


\section{List of Figures}

1.1 A schematic showing the scope of the proposed research. . . . . . . . . . 4

1.2 A schematic showing the proposed validation studies. . . . . . . . . . 5

2.1 A schematic of motivation for autonomous SHM. . . . . . . . . . 8

2.2 Key elements of SHM. . . . . . . . . . . . . . . . . . . . . . 9

2.3 Evolution of sensing technology in SHM. . . . . . . . . . . . . . . 11

2.4 Pattern recognition schemes for VDD. . . . . . . . . . . . . 15

2.5 A typical framework of ANN. . . . . . . . . . . . . . . . . . 18

2.6 The framework of SVM. . . . . . . . . . . . . . . . 20

2.7 The framework of an RF model with majority voting. . . . . . . . . . . . . 21

2.8 A schematic of the state-of-the-art CNN-based SHM operations [119]. . . . 31

3.1 (a) The harmonic signal $x(t)$ and its (b) CWT, and (c) SST. . . . . . . 42

3.2 Mixture of the sine signals: (a) time-histories of the signals (b) SST representation (c) Fourier spectra of the reconstructed signal. . . . . . . . . . . 43

3.3 The discrete change in frequency: (a) CCWT and (b) SST of the signal. . . 44

3.4 The progressive change in frequency: (a) CCWT and (b) SST of the signal. 45

3.5 Illustration of the mode decomposition using MEMD. . . . . . . . . . . . 48

3.6 SDOF Model. . . . . . . . . . . . . . . . . . . 51

3.7 Effect of frequency resolution under harmonic excitation (a) $\Delta f=0.38 \mathrm{~Hz}$ (b) $\Delta f=0.80 \mathrm{~Hz}($ c) $\Delta f=1.31 \mathrm{~Hz}$ (d) $\Delta f=1.97 \mathrm{~Hz}$, where $\Delta f$ represents the difference between undamaged and damaged frequency. . . . . . . . . . 
3.8 Effect of duration of damage under harmonic excitation (a) $\Delta t=5 \mathrm{sec}$, (b) $\Delta t=10 \mathrm{sec},(\mathrm{c}) \Delta t=20 \mathrm{sec}$, where $\Delta t$ represents the duration of linear reduction in the stiffness. . . . . . . . . . . . . . . . . . .

3.9 The simulated response with $\Delta f=1.31 \mathrm{~Hz}$, when there is a measurement noise of (a) $5 \%$, (b) 10\%, respectively; the simulated response with $\Delta t=10$ sec, when there is a measurement noise of (c) $5 \%$, (d) $10 \%$, respectively. . .

3.10 4-DOF Model. . . . . . . . . . . . . . . . . . . 55

3.11 Case 2: Fourier spectra of the IMFs obtained from MEMD. . . . . . . . . . 57

3.12 Case 2: SST of IMFs obtained from MEMD. . . . . . . . . . . . . . 57

3.13 Case 3: Fourier spectra of IMFs obtained from MEMD. . . . . . . . . . . . 58

3.14 Case 3: SST of IMFs obtained from MEMD. . . . . . . . . . . . . . . 58

3.15 The estimated modal damping ratio of (a) first, (b) second, (c) third, and (d) fourth modes for Case 2. . . . . . . . . . . . . . . . 59

3.16 Schematic of experimental setup for damage simulation with detachable mass and roller that move along the beam. (a) drop mass setup, (b) moving roller setup. ........................ 60

3.17 The mass drop experiment: (a) Fourier transform and (b) SST of the signal. 61

3.18 The moving roller experiment: (a) Fourier transform and (b) SST of the signal. . . . . . . . . . . . . . . . . .

3.19 (a) MIT Green building (b) sensor locations in the building (Sun and Buyukoz-

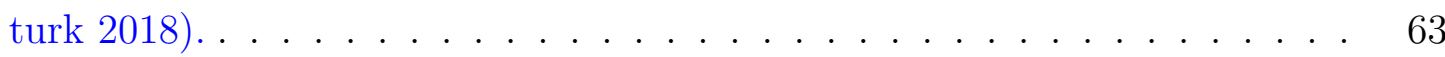

3.20 (a) Fourier spectra of IMFs (b) SST of IMFs obtained for ambient excitation. 64

3.21 (a) Fourier spectra of IMFs (b) SST of IMFs obtained for earthquake exci-

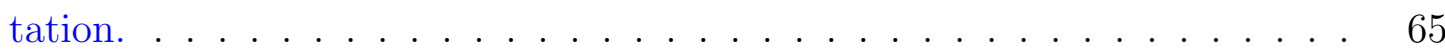

4.1 Fourier spectra of raw data of the 10-DOF model under various damage cases. 76

4.2 Fourier spectra of IMF-1 and IMF-2 of the 10-DOF model for C1. . . . . 78

4.3 Fourier spectra of IMF-1 and IMF-2 of the 10-DOF model for C2. . . . . 78

4.4 Fourier spectra of IMF-1 and IMF-2 of the 10-DOF model for C3. . . . . . 79 
4.5 $\Delta E_{a i}$ of IMFs for C2 subjected to Imperial Valley EQ using (a) 10 sensors, (b) 9 sensors, (c) 8 sensors, (d) 7 sensors, (e) 6 sensors, and (f) 5 sensors. $\quad 80$

4.6 $\Delta E_{a i}$ of IMFs for C3 subjected to Imperial Valley EQ using (a) 10 sensors, (b) 9 sensors, (c) 8 sensors, (d) 7 sensors, (e) 6 sensors, and (f) 5 sensors. . 80

$4.7 \Delta E_{a i}$ for C2 subjected to Borrego Mountain EQ using (a) 10 sensors, (b) 9 sensors, (c) 8 sensors, (d) 7 sensors, (e) 6 sensors, (f) 5 sensors. . . . . . . .

$4.8 \Delta E_{a i}$ for C3 subjected to Borrego Mountain EQ using (a) 10 sensors, (b) 9 sensors, (c) 8 sensors, (d) 7 sensors, (e) 6 sensors, (f) 5 sensors. . . . . . . 82

4.9 Schematic of the Z24 bridge. . . . . . . . . . . . . . . . 84

4.10 Schematic of the Z24 bridge piers and the selected sensors used in this study. 84

4.11 Lowering of the piers by cutting concrete $[106] \ldots \ldots \ldots$

4.12 The data of the Z24 bridge: (a) time history and (b) Fourier spectrum of the response of the undamaged pier (sensor-421), (c) time history and (d) Fourier spectrum of the damaged pier (sensor-521) . . . . . . . . 86

$4.13 \Delta E_{a i}$ for $20 \mathrm{~mm}$ lowering of various sensor cases using, (a) 8 sensors, (b) 6 sensors, (c) 4 sensors. . . . . . . . . . . . . . . . . . . 87

$4.14 \Delta E_{a i}$ for $40 \mathrm{~mm}$ lowering of various sensor cases using, (a) 8 sensors, (b) 6 sensors, (c) 4 sensors. . . . . . . . . . . . . . . . . . 87

$4.15 \Delta E_{a i}$ for $95 \mathrm{~mm}$ lowering of various sensor cases using, (a) 8 sensors, (b) 6 sensors, (c) 4 sensors. . . . . . . . . . . . . . . . . . . 88

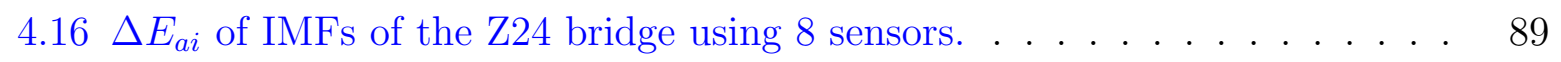

$4.17 \Delta E_{a i}$ of IMFs of the Z24 bridge using 6 sensors. $\ldots \ldots \ldots \ldots$

$4.18 \Delta E_{a i}$ of IMFs of the Z24 bridge using 4 sensors. . . . . . . . . . 90

5.1 1D CNN architecture used in this study. . . . . . . . . . . . . . 98

5.2 Extracting data sequences of windows from the vibration data using 1D CNN architecture. . . . . . . . . . . . . . . . . . . . . 101

5.3 Data pipelines for training the proposed 1D CNN network and obtaining predictions for a given time-series. . . . . . . . . . . . . . . 103 
5.4 Sensor placement for data acquisition. . . . . . . . . . . . . . . 107

5.5 Performance evaluation of 1D CNN based on window size for (a) pier settlement and (b) rupture of tendons. . . . . . . . . . . . . . 112

5.6 Performance of 1DCNN by windowing of the data of pier settlement (a) series-ROC, (b) windowed-voted ROC, (c) series-PR, (d) windowed-voted PR.114

5.7 Performance of $1 \mathrm{DCNN}$ by windowing of the data of rupture of tendons (a) series-ROC, (b) windowed-voted ROC, (c) series-PR, (d) windowed-voted PR.115

5.8 Schematic showing the sensor location and their numbers used in the analysis.116

5.9 Damage localization for pier settlement with two damage levels, $(a, b, c): 20$ $\mathrm{mm}$ and $(d, e, f): 40 \mathrm{~mm} . \ldots \ldots \ldots \ldots \ldots \ldots$

5.10 Damage localization for pier settlement with two damage levels, $(a, b, c): 80$ $\mathrm{mm}$ and $(d, e, f): 95 \mathrm{~mm} . \ldots \ldots \ldots \ldots \ldots \ldots$

5.11 Damage localization for the pier settlement. . . . . . . . . . . . . . 119

5.12 Damage localization for the rupture of tendons. . . . . . . . . . . . 120

6.1 The typical internal structure of an LSTM cell [33] . . . . . . . . . . . 130

6.2 Extracted sequences of windows from the vibration signals in the LSTM network. . . . . . . . . . . . . . . . . . . . 133

6.3 Data pipelines for training and prediction of the LSTM network from the acceleration response. . . . . . . . . . . . . . . . 135

6.4 QUGS testbed, where the joints are numbered from 1 to 30 [4]. . . . . . 141

6.5 Undamaged and damaged state of the girder and beam joint in the QUGS [4].141

6.6 Performance evaluation of the proposed LSTM model based on window size in the QUGS data. . . . . . . . . . . . . . . . . . . . . 143

6.7 Performance evaluation for random weight initialization of QUGS data: (a) Accuracy, and (b) FNR. . . . . . . . . . . . . . . . . . 144

6.8 Performance of the LSTM model on the QUGS data: (a) ROC in individual windows, (b) ROC in voted series, (c) PR in individual windows, (d) PR in voted series. . . . . . . . . . . . . . . . . . . 146 
6.9 Damage localization probabilities in the QUGS data for scenarios where the damaged joint is: (a) Joint 1 [1,1], (b) Joint 4 [4,1], (c) Joint 12 [2,3], (d) Joint $15[5,3]$, (e) Joint $23[3,5]$, and (f) Joint $28[3,6] \ldots \ldots$. . . . . . . 147

6.10 Performance evaluation of the proposed LSTM model based on window size for (a) rupture of tendons, and (b) pier settlement. . . . . . . . . . . . 150

6.11 Performance evaluation of LSTM for random weight initialization for various damage cases in the Z24 bridge: (a) accuracy for pier settlement, (b) FNR for pier settlement, (c) accuracy for rupture of tendons, (d) FNR for rupture of tendons. . . . . . . . . . . . . . . . . . . . 151

6.12 Performance of the proposed LSTM model in the Z24 bridge for pier settlement: (a) ROC in individual windows, (b) ROC in voted series, (c) PR in individual windows, $(\mathrm{d}) \mathrm{PR}$ in voted series. . . . . . . . . . . . .

6.13 Performance of the proposed LSTM model by windowing the data of the Z24 bridge in case of rupture of tendons: (a) ROC in individual windows, (b) ROC in voted series, (c) PR in individual windows, (d) PR in voted series.154

6.14 Damage localization for lowering of pier for three damage levels, where, $(a, b, c)$ are for $20 \mathrm{~mm}$ lowering of piers, $(d, e, f)$ are for $40 \mathrm{~mm}$ lowering of piers, $(g, h, i)$ are for $95 \mathrm{~mm}$ lowering of piers. . . . . . . . . . . . 156

6.15 Damage localization for lowering of pier, where legend shows the amount of pier-settlement. . . . . . . . . . . . . . . 157

6.16 Damage localization for rupture of tendons, where the legend shows the number of tendon ruptures. . . . . . . . . . . . . . . . . . 158 


\section{List of Tables}

3.1 Identified frequencies $(\mathrm{Hz})$ for the simulated damage cases. . . . . . . . . 56

3.2 Identified results $(\mathrm{Hz})$ based on the measurement along East-West direction. 65

4.1 Varying level of damage in the first floor and the associated acronyms. . . 75

4.2 The first five modal frequencies of the 10-DOF model for various damage cases and their percentage reduction with respect to $\mathrm{C} 1 . \ldots \ldots$

4.3 Modal energies $\left(E_{i j}(f)\right)$ of IMF-1 and IMF-2 of the 10-DOF model subjected to Imperial Valley earthquake. . . . . . . . . . . . . . . . . . . 77

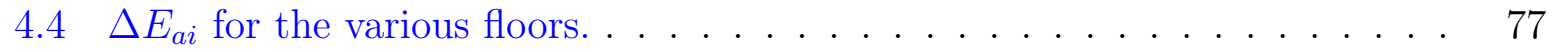

4.5 Damage localization accuracy of the 10-DOF model using the limited number of sensors. . . . . . . . . . . . . . . . . . . . . . . 81

5.1 Confusion matrix for a multiclass problem. . . . . . . . . . . . . . . 104

5.2 Description of various performance metrics. . . . . . . . . . . . . . 105

5.3 Description of the multiclass damage scenarios and the class labels. . . . . 108

5.4 Hyper-parameters used in 1D CNN for tuning by random search algorithm. 109

5.5 Optimal configuration of the hyper-parameters of the selected 1D CNN. . . 110

5.6 Random initialization of weights . . . . . . . . . . . . . . . 111

5.7 Training and testing performance of 1D CNN . . . . . . . . . . 113

6.1 Confusion matrix for a binary classification problem. . . . . . . . . . 137

6.2 Description of the selected performance metrics. . . . . . . . . . . . 138 
6.3 Hyperparameters used for tuning LSTM by random search algorithm. . . . 142

6.4 Optimal configuration of the LSTM hyperparameters for QUGS data. . . . 142

6.5 Multiclass problem description for two damage scenarios along with the class label. . . . . . . . . . . . . . . . . . . . . . . 149

6.6 Optimal configuration of the LSTM hyperparameters for the Z24 bridge benchmark data. . . . . . . . . . . . . . . . . . 150 


\section{Chapter 1}

\section{Introduction}

\subsection{General Introduction}

Civil infrastructure is built to perform safely and remain operational during its design life. However, due to various factors such as operational and environmental conditions, the structures accumulate damage and deteriorate with time. Any imperfections, local/global defects, or conditions of the structure that impair operating conditions of civil infrastructure are considered damage to structures. Damage detection has been a critical research area since the dawn of the 20th century. The most common type of damage in the structures is induced due to external environmental factors such as earthquakes, temperature, high-speed wind and turbulence, floods or freeze-thaw cycles. Due to their inherent flexibilities, slender structures such as tall buildings and long-span bridges are more prone to catastrophic failure if damages are not monitored continuously. Integrity and safety of these structures can be ensured with continuous monitoring and maintenance. In the last 
few decades, several damage detection techniques have been developed for global, local, and online monitoring of damages using Structural Health Monitoring (SHM).

There are various damage detection methods (i.e., visual inspection, static and vibration measurements, and image-based techniques) depending on their mathematical and modelbased approach towards data analysis. In particular, Vibration-based Damage Detection (VDD) methods $[11,136,29,129,64]$ can identify and localize damages in structure by using cost-effective sensors such as accelerometers. Many VDD techniques based on changes in modal parameters (frequency, mode shapes, and damping) or their variants (mode shape curvature, modal strain energy, and flexibility matrix) are explored in the past for damage identification. However, these methods require a large number of sensors, which demand significant time and expense, and often suffer from limited access to critical locations. Several studies were conducted in the past based on sensitivity of modeshape and frequencies, and these methods are prone to error accumulation and other uncertainties leading to unreliable damage localization. It is critical to develop robust and cost-effective VDD methods based on limited sensors capable of keeping the same information as a large array of sensors while providing high accuracy in damage detection and localization.

There has been considerable research on system identification using limited sensors; however, damage detection using limited measurements is still unexplored. Non-sparse methods have gained interest as non-parametric methods [1, 57, 108]. Unlike non-sparse methods, sparse methods utilize time-frequency (TF) methods for sparse representation of the signals and subsequent pattern recognition for damage identification [140, 146, 142, 144]. However, damage localization is not given its due attention under limited sensor mea- 
surements [49]. In addition to limited measurements, most of the existing VDD methods lack automation, require user intervention, and are dependent heavily on model parameters. It is vital to introduce damage detection methods capable of identifying and localizing damage in structures irrespective of the material, type, and nature of loading. Traditional signal processing methods are well established but lack automation and require manual damage feature extraction in case of multiclass damages (e.g., pier settlement, tendon failure or defects in girders of bridges). Modern pattern recognition requires evaluation of model parameters and are prone to data overfitting. There is a need for a diagnostic tool that can learn continuously and provide autonomous monitoring of civil infrastructure while overcoming drawbacks of traditional VDD methods. In this thesis, basis-free TF modal decomposition method and deep learning-based neural networks are explored for autonomous and continuous VDD of structures using limited number of sensors.

\subsection{Scope of the Thesis}

The scope of this thesis is bounded in search of cost-effective VDD schemes by implementing basis-free TF method, namely, Multivariate Empirical Mode Decomposition (MEMD) and deep learning-based neural networks, namely, one-dimensional convolutional neural network (1D CNN) and Long Short-Term Memory (LSTM) to provide an autonomous, continuous, and intervention-free damage detection and localization using limited measurements. In addition to the theoretical development, this thesis is also intended to validate the proposed formulations and assumptions using a suite of numerical, experimental and full-scale studies. Both the proposed research and validation studies of this thesis are 
summarized in Fig. 1.1 and 1.2, respectively.

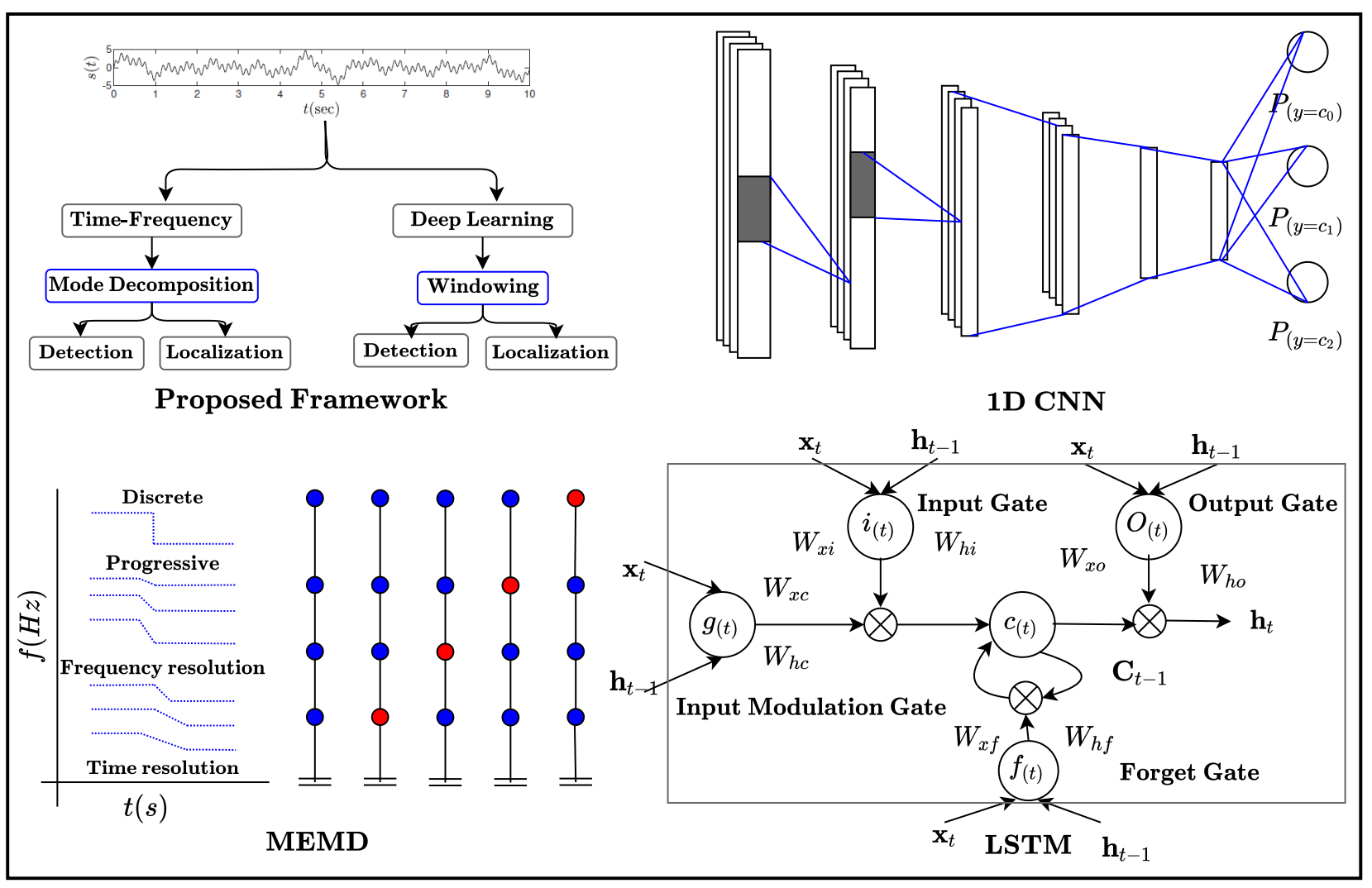

Figure 1.1: A schematic showing the scope of the proposed research.

\subsection{General Objectives}

The general objectives of the current research is to accomplish following broad tasks:

- To develop a basis-free algorithm for damage detection and localization, that are insensitive to measurement noise and severity of damage.

- To explore the applicability of limited sensors for damage localization to develop cost-effective VDD for large-scale structures.

- To investigate feature-free multiclass VDD framework using limited training data, 
suitable for remote and autonomous SHM.

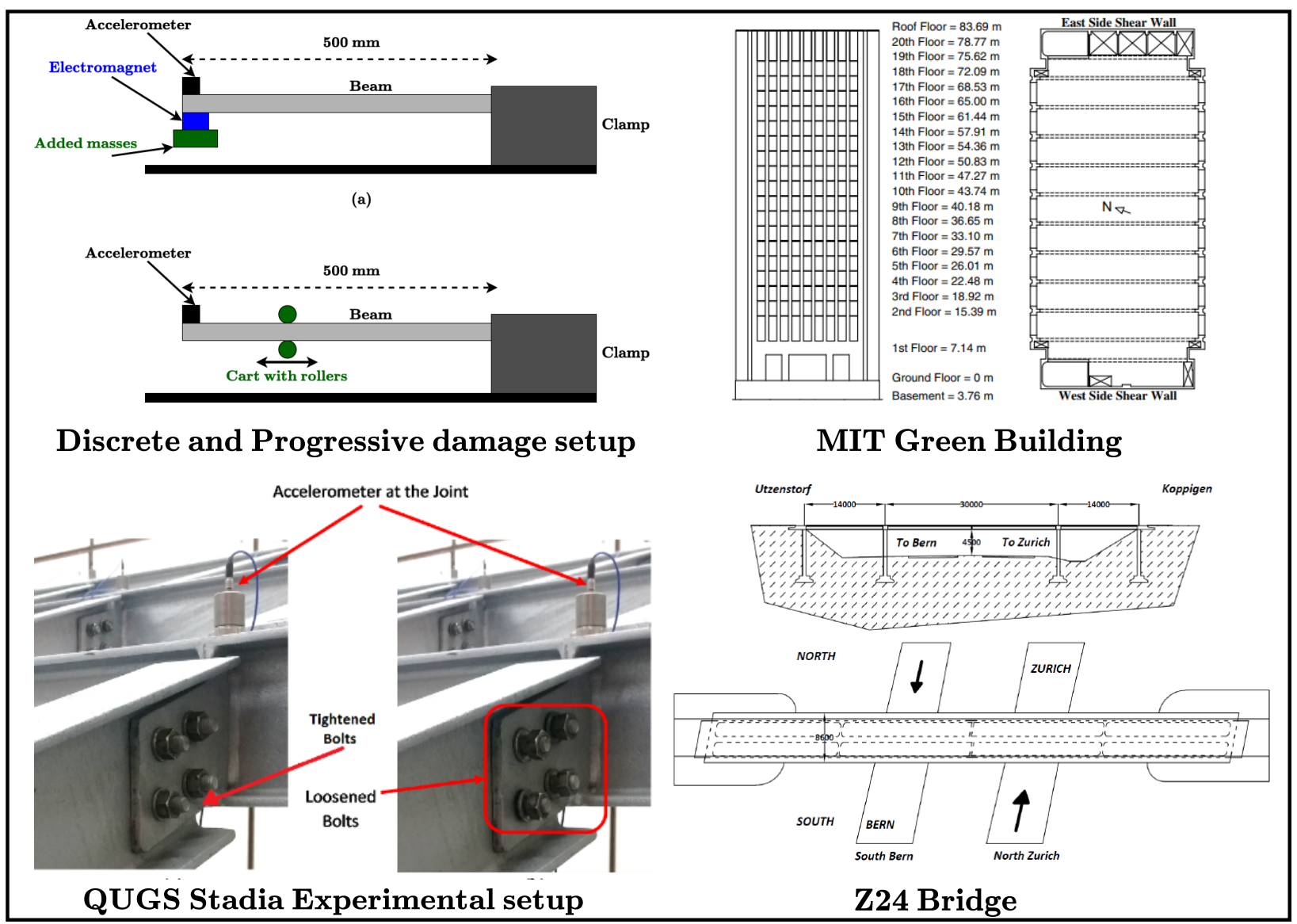

Figure 1.2: A schematic showing the proposed validation studies.

\subsection{Organization of the Thesis}

The thesis is organized as follows:

I. A generic introduction and thesis objectives along with organization of the thesis is provided in Chapter 1.

II. Chapter 2 provides a brief introduction and literature review of conventional and modern VDD methods, along with their limitations and challenges. 
III. Synchrosqueezing transform-based time-varying VDD algorithm is proposed in Chapter 3, where the proposed algorithm is evaluated using both discrete and progressive damage.

IV. In Chapter 4, Multivariate Empirical Mode Decomposition-based VDD is proposed to locate the damage using limited sensor measurements.

V. A novel 1D CNN technique is proposed to perform multiclass damage identification in Chapter 5.

VI. In Chapter 6, several challenges posed by 1D CNN are further addressed by using a newer class of Long Short-term Memory networks to undertake multiclass damage identification using limited training datasets.

VII. The key conclusions, research contributions, and future directions of this thesis are discussed in Chapter 7. 


\section{Chapter 2}

\section{Literature Review}

A brief introduction to conventional and modern damage detection literature is provided in this chapter. Conventional techniques such as time-frequency methods and pattern recognition algorithms including time-series analysis and machine learning techniques have proved to be highly efficient in detecting damages in structures. However, these techniques have several limitations that hinder their direct implementation in providing an autonomous framework for damage detection and localization in civil infrastructure. In this context, several modern techniques such as advanced time-frequency methods and artificial intelligence algorithms have been reviewed and their existing gap areas are discussed. Based on these gap areas, the key objectives of this thesis are identified at the end of this chapter.

\subsection{Background}

In today's age of globalization and climate change, large-scale infrastructure such as buildings and bridges undergoes rapid ageing and a noticeable reduction in its design life 
[29]. Exponentially increasing population and traffic, unpredictable natural calamities, and human-made damages have resulted in frequent disruptions in their operational state as well as numerous cases of catastrophic failures worldwide. The United States of America reported the current condition of the infrastructure through a letter grade, and it has been consistent with a low grade of D+ [13]. The Canadian infrastructure has been ranked along the same lines; it has been reported that it would cost $\$ 1.1$ trillion to replace all assets with $\$ 141$ billion assets that are in 'very poor' condition [37]. It can be inferred that the development of efficient strategies for continuous structural monitoring is of paramount importance for the ageing structures, as illustrated in Fig. 2.1.

\section{DESIGN LIFE}

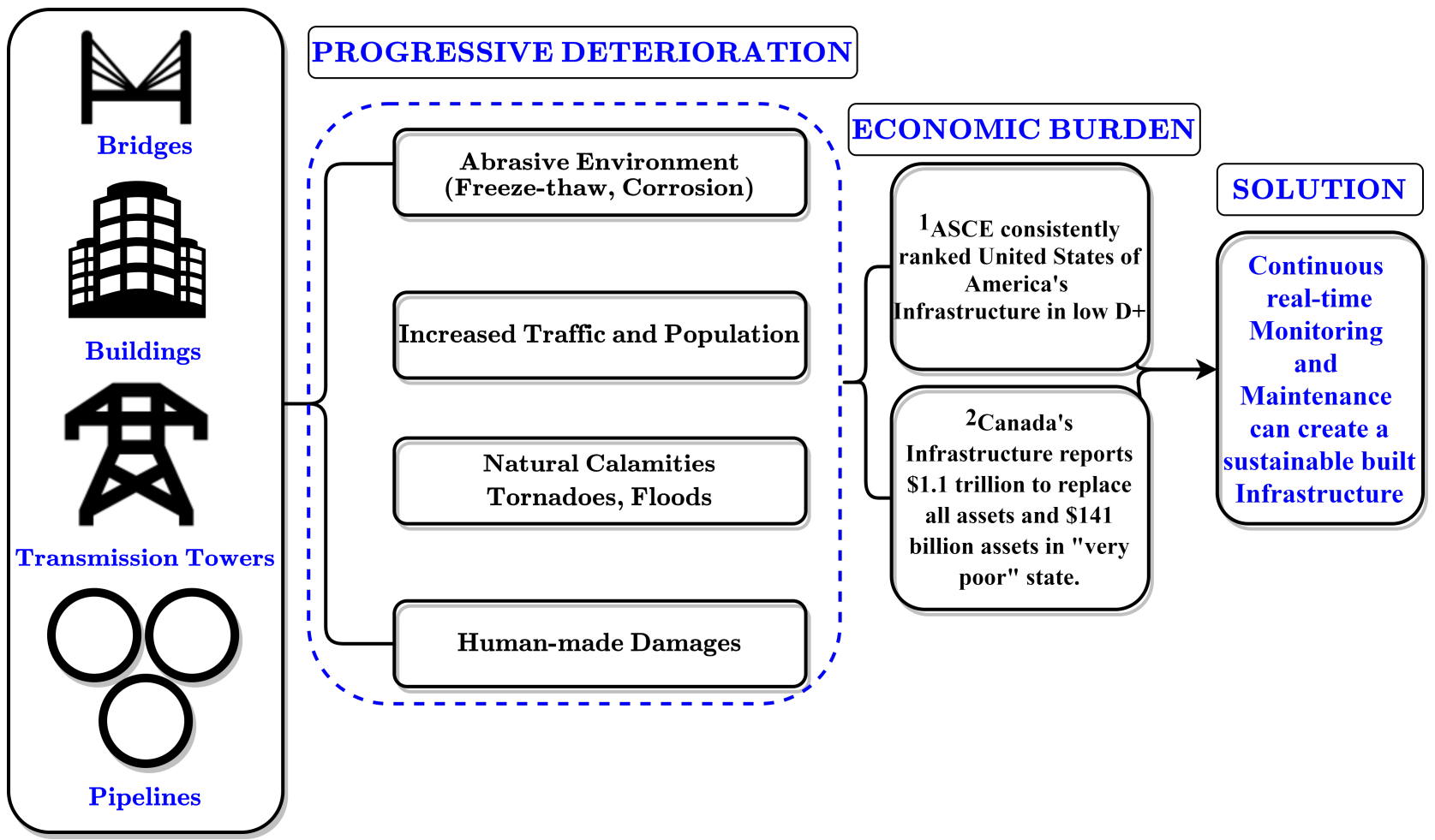

Figure 2.1: A schematic of motivation for autonomous SHM.

The conventional structural monitoring approach is to employ a well-trained structural 
inspector to inspect the structure, identify defects and implement appropriate maintenance strategies. However, such manual structural assessment is subjective, error-prone and laborious, incurring a significant portion of the annualized maintenance budget. Structural Health Monitoring (SHM) provides a sensor-driven real-time inspection technology to address these challenges of manual visual inspection. Vibration-based SHM techniques [47, 43, 100, 69] offer viable options for tracking time-varying behaviors of the ageing structures based on the measured data.

INSTRUMENTATION

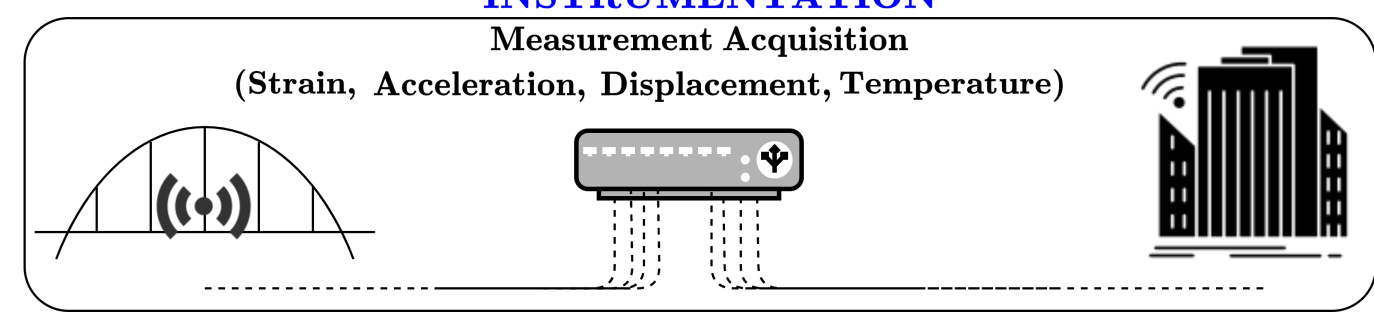

DATA MANAGEMENT

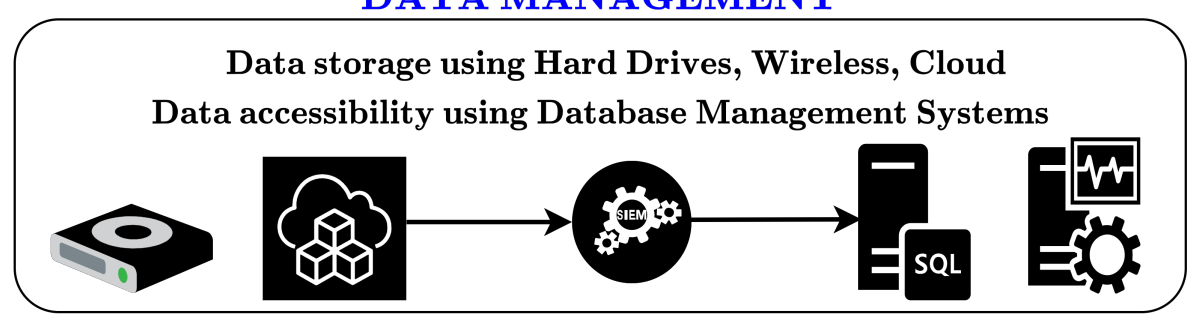

DATA INTERPRETATION

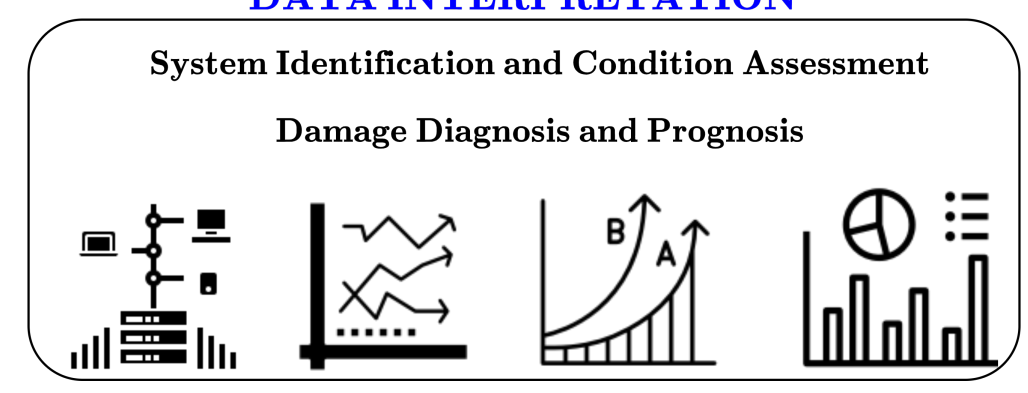

Figure 2.2: Key elements of SHM.

In general, vibration-based SHM consists of four key elements: instrumentation and data acquisition, condition assessment, damage detection, and damage prognosis, as illus- 
trated in Fig. 2.2. The structures are first instrumented with various sensors to acquire valuable measurements (such as, acceleration and displacement). The acquired data is then analyzed to assess the condition of the structure and detect any changes in the structures using various system identification and damage detection methods. Maintenance and retrofitting strategies are then adopted to estimate the remaining useful life and improve the structural condition using various prognosis techniques.

There have been continuous improvements in sensors and sensing technology as they constitute a significant portion of cost during SHM applications and contribute to the overall accuracy of the condition assessment. Traditionally, wired sensors are used as a dense array distributed over the structure to acquire most of the vibration information. However, they are not a cost-effective and viable option for large-span bridges or tall-buildings due to labor-intensive cable installation. The setbacks of wired sensors were envisioned to solve using smart wireless sensors $[90,27,5]$. The capabilities of wireless sensors and their local processing under the decentralized framework were much later exploited [122, 89, 107]. Recently, modern sensors such as cameras, robotic sensors, smartphones, and drones have been used for SHM through the processing of images and videos [45, 120], as illustrated in Fig. 2.3. However, the acquired data is only valuable if the hidden structural information and damage characteristics are accurately assessed from the measured vibration data. Irrespective of the type of sensors, the accuracy of the existing damage detection and localization methods largely depend on the availability of a large number of sensors and high-quality data sets, which form a major hindrance to SHM of large-scale structures such as, buildings and bridges. 

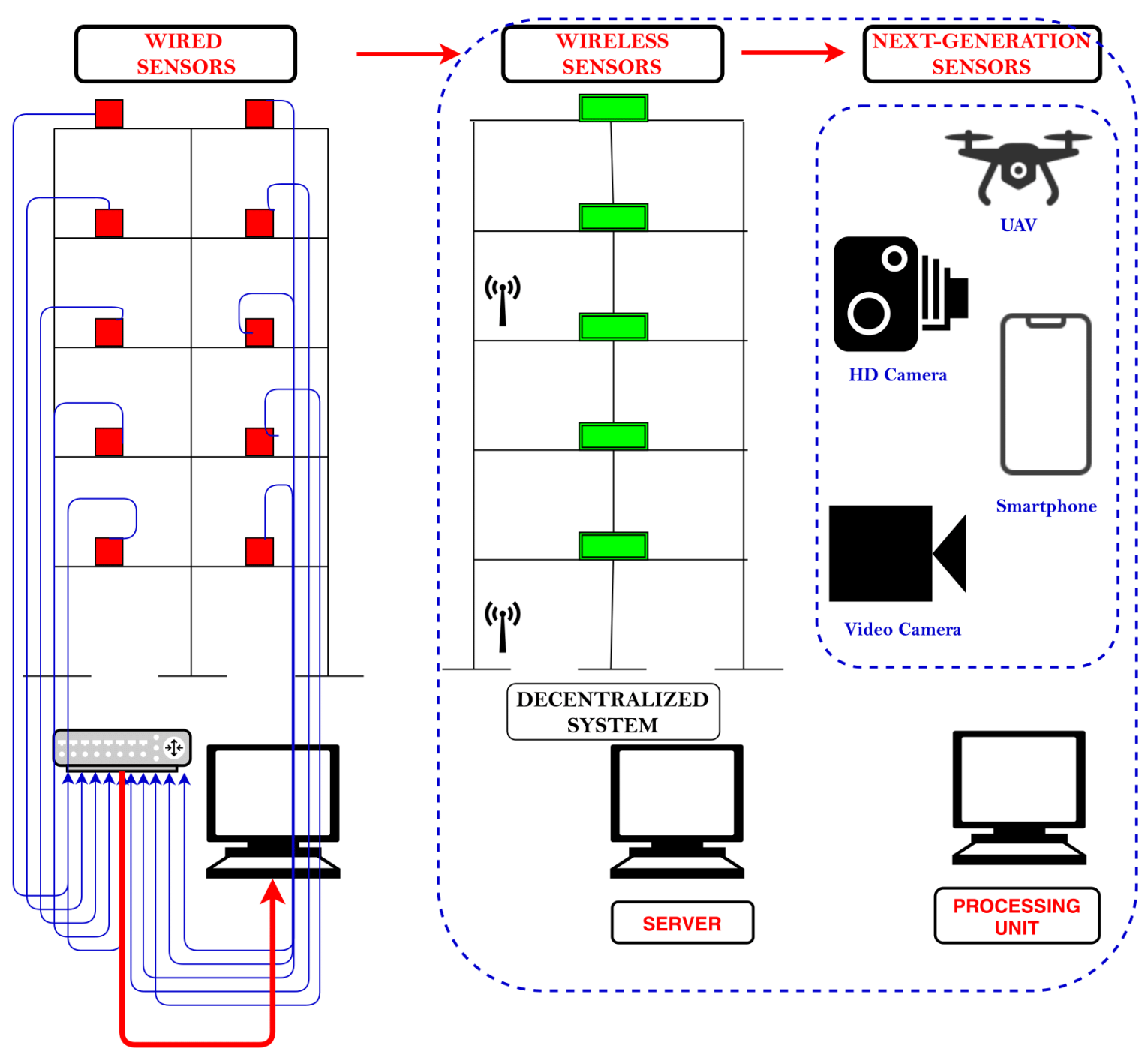

Figure 2.3: Evolution of sensing technology in SHM.

In a structure, the damage is initiated due to changes in the system's material, geometric properties, or boundary conditions. As these properties change with time, the structure is mathematically modeled as a time-varying system [154, 34]. The basic idea behind vibration-based damage detection (VDD) techniques is to identify changes in vibration characteristics of modal parameters (i.e., frequency, damping and modeshapes) or physical parameters (i.e., stiffness, damping or mass) using the pattern recognition algorithms. Any change in such parameters is inferred as damage in structures. VDD has 
been a crucial topic of research among SHM researchers for the last several years. In the following section, various VDD techniques based on TF, pattern recognition, and artificial intelligence techniques are reviewed.

\subsection{Traditional VDD Methods}

The time-domain methods only provide information about the time characteristics of the systems. These methods are easier to implement; however, they are prone to noise contamination and environmental factors, which offers challenges for large structures [23]. Frequency-domain methods provide information about the frequency characteristics of the systems. Although frequency changes in the structure can be associated with damage, discrete changes in natural frequencies may not be sufficient for unique identification of the location of structural damage as a crack at two different locations will have the same frequency change irrespective of its location [110]. Another critical limitation of frequency changes caused by damage is usually minimal and may be suppressed in the changes caused by environmental and operational conditions. Unlike time- and frequency-domain techniques, Time-Frequency (TF) method [95] can identify the signal's frequency components and apprehend their time-variant features.

\subsubsection{Time-frequency methods}

The TF methods have the ability to perform localized analysis of a signal, i.e., to zoom-in on any interval of time or space. The TF methods can reveal or track the hidden information of the data that standalone time or frequency domain techniques fail to detect. This 
property is particularly important for VDD.

\section{(a) Short-Time Fourier Transform}

The Short-Time Fourier Transform (STFT) [35] is the classical modified version of the Fourier transform that allows to analyze nonstationary signals in TF domain. STFT is based on the Fourier transform of a fixed windowed signal. STFT is given by,

$$
s_{i}(\omega)=\int_{-\infty}^{\infty} s(\tau) h(\tau-t) e^{-2 j f \pi \tau} d \tau
$$

where $h(\tau-t)$ is an approximately chosen window function that emphasizes the signal around time $t$. This windowing technique analyzes only a small segment of the signal at a time $t$. The STFT maps a signal into a $2 \mathrm{D}$ function of time and frequency. The TF resolution of the STFT technique is inversely related to the window length. Increasing the window length increases the frequency resolution while at the same time, it reduces the frequency tracking capability of the representation. One of the major limitations of STFT is that a high resolution in time and frequency cannot be obtained simultaneously, as the selected window size remain the same for all frequencies.

\section{(b) Wigner-Ville Distribution}

The Wigner-Ville (WVD) distribution [137] can be derived by generalising the relationship between the power spectrum and the autocorrelation function for a nonstationary, time-variant process. The Wigner-Ville distribution provides a high-resolution TF representation of a signal. It is a nonlinear TF transform, and for a continuous signal $x(t)$, the 
Wigner-Ville distribution is defined as,

$$
W_{x}(t, f)=\int_{-\infty}^{\infty} x\left(t+\left(\frac{\tau}{2}\right)\right) \overline{\left(t-\left(\frac{\tau}{2}\right)\right)} e^{-2 j f \pi \tau} d \tau
$$

Similar to STFT [39], WVD [12] has been explored in many SHM applications. However, WVD has an unwanted cross-product, which poses challenges to reconstruct the signal. Therefore, conventional TF analysis suffers from a trade-off between time and frequency resolution. Moreover, vibration data associated with VDD attributes highly nonlinear and nonstationary behavior due to structural damage, which cannot be handled using WVD.

\subsubsection{Pattern recognition methods}

VDD can also be posed as a pattern recognition problem. The objective of any pattern recognition-based VDD algorithms is to distinguish between patterns present in the identified features related to the undamaged and damaged structure under the same operational and environmental circumstances. Various time-series modeling and Machine Learning (ML) techniques have been employed to identify the key features used in VDD, as shown in Fig. 2.4. 


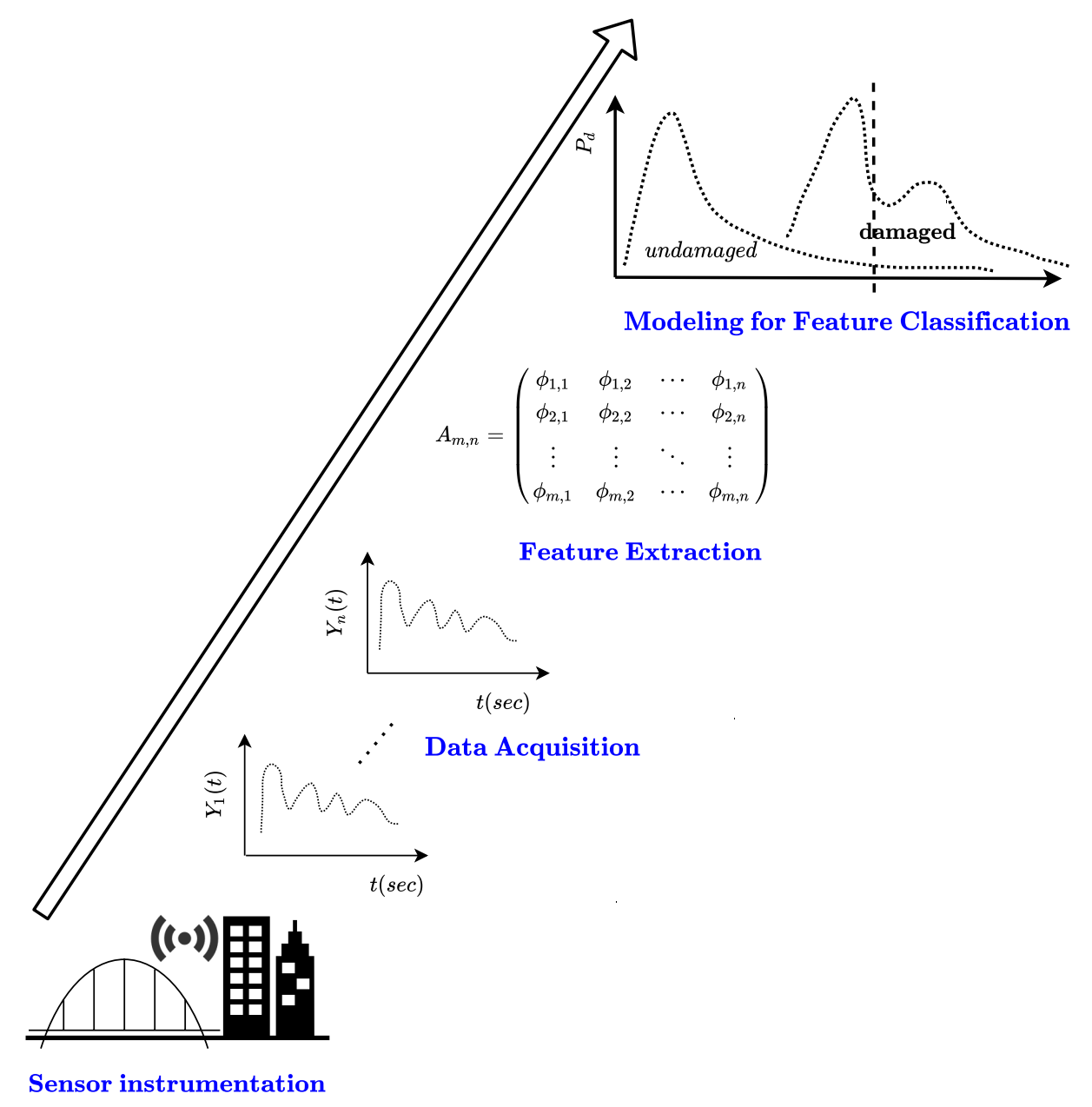

Figure 2.4: Pattern recognition schemes for VDD.

\section{(a) Time-series modeling}

Time-series modeling has been one of the popular methods for damage identification in structures. The basic principle is to model a time-series, evaluate model coefficients and calculate residual errors; any deviation in those coefficients or residual error can be inferred as damage in the structure. Primarily, auto-regressive (AR) models with several variants have been used in damage analysis. The AR models assume the measurements have noise, and modeling error is sampled from Gaussian distribution. The AR models have several variants that are extensively used in SHM and for damage detection, such as auto-regressive 
moving-average (ARMA), AR-integrated moving-average (ARIMA), and AR model with exogenous input (ARX). The AR modeling of a sensor data is given by,

$$
x_{t}=\sum_{j=1}^{p} \phi_{j} x(t-j)+e_{x}(t)
$$

where $x_{t}$ is the representation of the measured signal at $t, \phi_{j}$ are the AR coefficients or model parameters, $e_{x}(t)$ is a white noise or residue term. In this model, Yule-Walker equation can be used to solve for the coefficients [24] and Akaike's information criteria [86] can be used to evaluate the optimum model order. Unlike in the AR models, ARMA model represents the noise part in a regressive manner as shown below,

$$
x_{t}=\sum_{j=1}^{p} \alpha_{i} x(t-i)+\sum_{j=1}^{q} \beta_{j} e_{x}(t-j)+e_{x}(t)
$$

where $\alpha_{i}$ and $\beta_{j}$ are the $i$-th AR and MA coefficient, respectively and $p$ and $q$ are the model orders of the AR and MA processes. The AR part of order $p$ describes the model dynamics, while the MA part of order $q$ is related to the external noise and the white noise excitation and ensures the stationarity of the system response.

In the SHM literature, time-series modeling have been extensively used by the researchers. For example, [85] evaluated various distance measures for damage detection, where Mahalanobis Distance (MD) showed promising results. [97] showed that AR coefficients with higher-order have highest sensitivity to damages. The significant drawback of AR model is its incapability to represent nonlinearity and assumption of stationary timeseries. [26] extracted ARMA and ARIMA coefficients from acceleration and strain data 
as damage features. [53] employed ARX models by using free acceleration responses to extract damage-sensitive features. The authors developed two different techniques, first, using $\beta$-term coefficients of the ARX models for noise-free models. Secondly, the fit ratio of the ARX model was considered as a damage sensitive feature to include noise and model complexity. [52] extended the approach by using random decrement (RD) for ambient vibration case to obtain a pseudo-free vibration response. RD eliminated the effects of the exogenous input by normalizing the ambient vibration data before constructing the AR models. In addition to damage detection, ARX models were further used for sensor fault localization [87]. Several statistical features such as mean, standard deviation, variance, kurtosis, skewness, root-mean-square, quantile and cross-covariance function were used with various models $[101,38,148,153]$. A comparison of features between the undamaged and damaged state of a structure was used to identify and localize damage in structures. Various improved damage-sensitive features based on time-series modeling were also extracted, which include the standard deviation of residual error, F-statistic, calculated by testing null hypothesis [117].

\section{(b) Machine Learning techniques}

ML methods include a suite of supervised learning and unsupervised learning techniques, where supervised learning required labeled data of both undamaged and damaged dataset to establish a statistical model during the training process. However, the labeled dataset is not required for unsupervised learning algorithms. ML algorithm is evaluated on its capability to predict the new input data with high accuracy. Out of several variants of ML techniques, Artificial Neural Network (ANN), Support Vector Machine (SVM), Random 
Forest $(\mathrm{RF})$, and clustering methods have been extensively used in VDD.

\section{ANNs}

ANNs are inspired by the biological neuron network and how information is learned and transferred through the human brain. A nerve cell can be considered as a biological neuron that transmits information through electrochemical signals to synaptic terminals. The signal is transmitted when there is enough concentration of information in the nerve cell. The analogy can be interpreted for ANN. An artificial neuron acts as a processing unit that first aggregates the incoming signals via a weighted sum, and then an activation function is applied to generate an output.

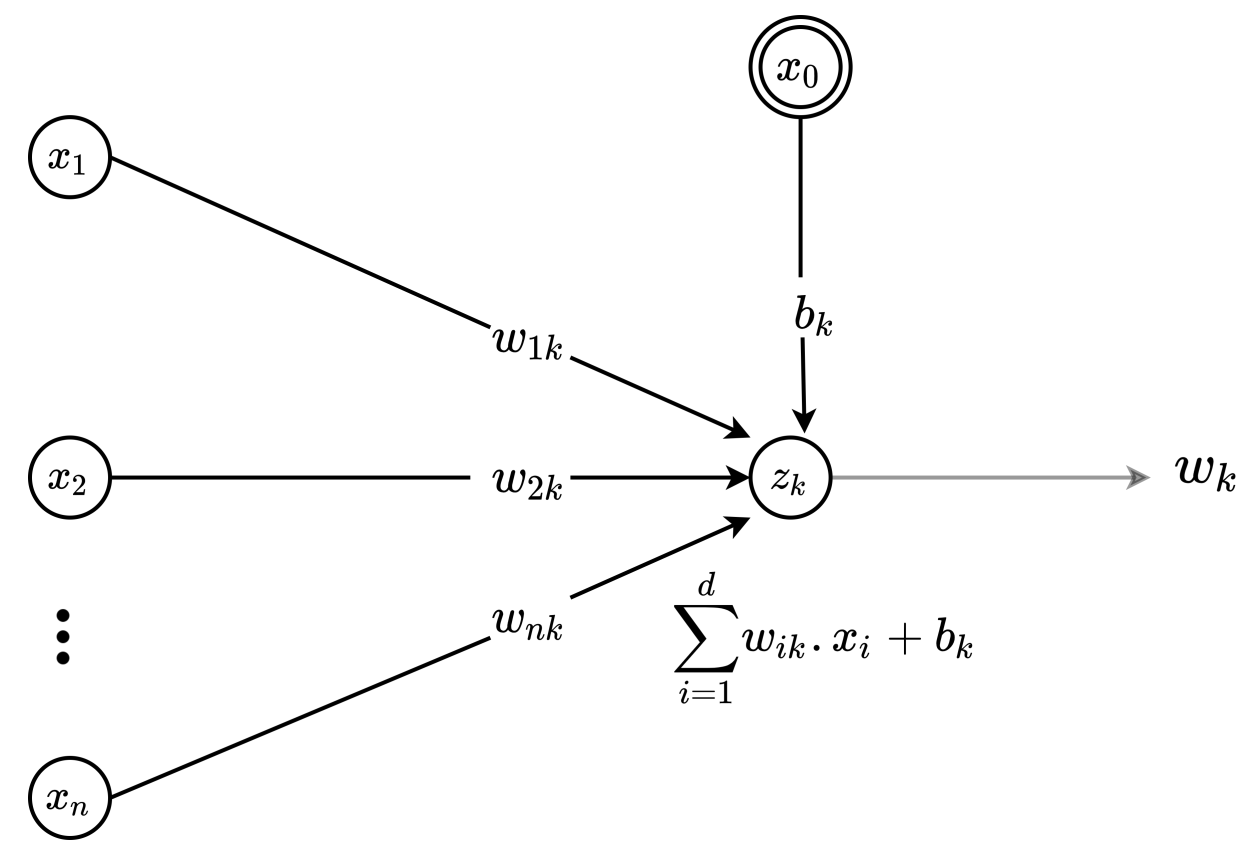

Figure 2.5: A typical framework of ANN.

The ANN process is presented in Fig. 2.5. It can be observed that neural weight $\left(w_{n k}\right)$ and bias vector $\left(b_{k}\right)$ aggregate to a cumulative net input from multiple neurons and ac- 
tivation functions such as Rectified Linear Unit (ReLU), sigmoid, softmax or hyperbolic tangent function are used to activate the neural networks to transfer information to subsequent layers. The activation functions differ in the way they transfer the output from each neuron. For example, sigmoid is a better choice where the output layer is a binary ( 0 or 1) classifier, whereas tanh is a better choice as it is a continuous function and its output ranges between -1 and +1 . On the other hand, ReLU does not activate if the net input is less than or equal to zero. Softmax is a prevalent activation function, and it is used at the output layer as unlikely other functions, it does not depend only on the net input of a neuron $n$ but depends on net signal at all other neurons in the output layers.

\section{$S V M$}

SVM aims to separate two classes of the data by finding an optimal hyperplane that maximizes the margin between the two classes and minimizes the misclassification error. The anatomy of the hyperplane and separating distances is shown in Fig. 2.6. SVM has gained popularity for damage detection due to its capability to model nonlinear, high dimensional, and small sample problems [36]. For a binary classification dataset with $n$ points $x_{i}$ in a $d$-dimensional space. A hyperplane function $h(x)$ can be defined as,

$$
h(x)=w_{1} x_{1}+w_{2} x_{2}+w_{3} x_{3}+\ldots+w_{d} x_{d}+b
$$

where $w$ is a $d$-dimensional weight vector, and $b$ is a scaler, called the bias. For points that lie on the hyperplane, $h(x)=0$. The points shown in circles can be thought of as one class, and triangular points represent the other class. The hyperplane divides the space 
into two half-spaces. The unit weight vector $\frac{w}{\|w\|}$ is orthogonal to the hyperplane. The directed distance of the origin to the hyperplane is $\frac{b}{\|w\|}$.

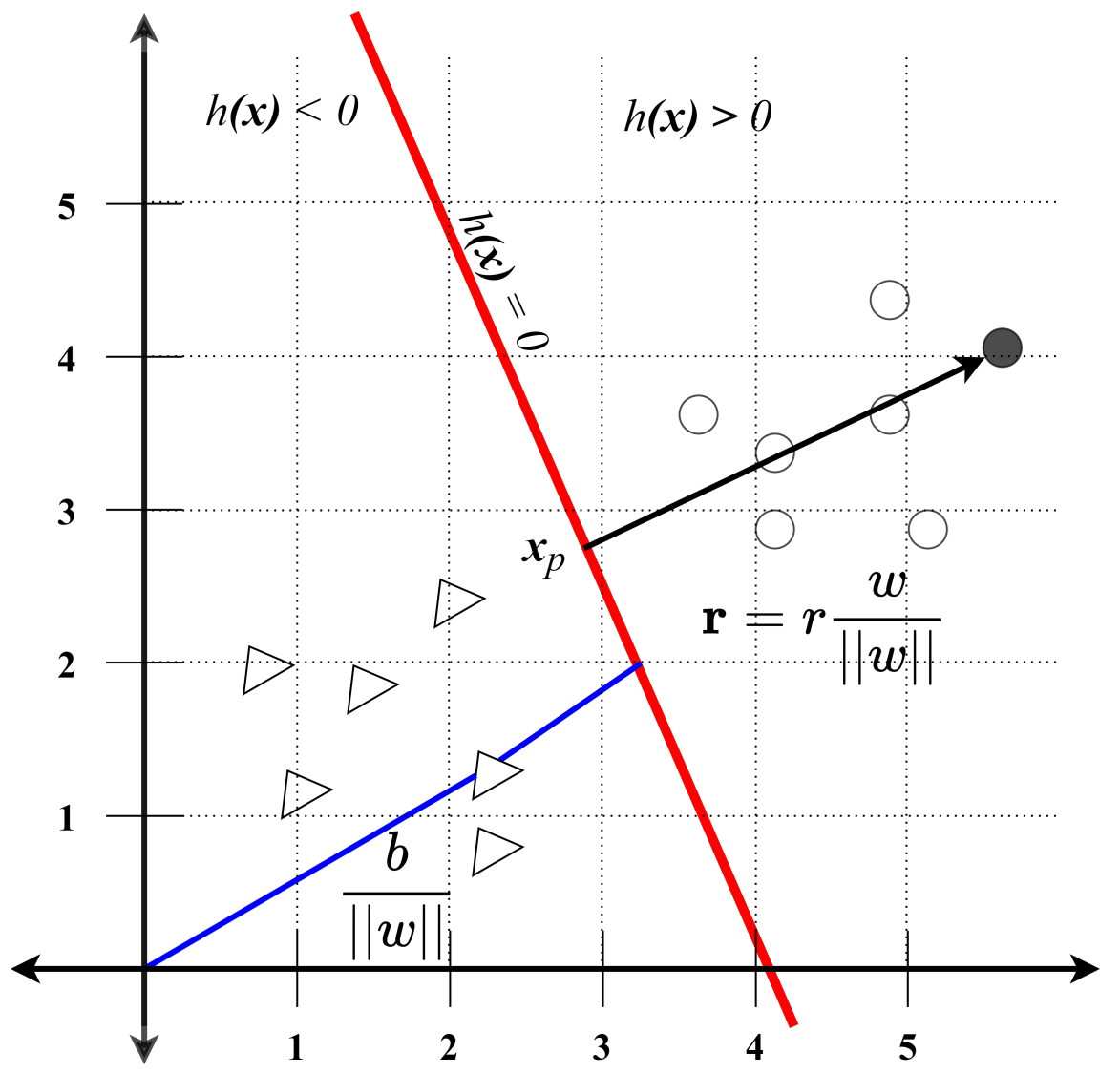

Figure 2.6: The framework of SVM.

\section{$\boldsymbol{R F}$}

$\mathrm{RF}$ is an ensemble method that combines multiple classifiers to improve classification performance. It is a decision tree-based method that differs from traditional decision tree algorithms by combining many decision trees and alleviates the overfitting. In classification problems, the goal is to create a model that predicts a target variable's value based on several input variables. A decision breaks down a dataset into smaller and smaller subsets while at the same time an associated decision tree is incrementally developed. The final 
result is a tree with decision nodes and leaf nodes. RF uses multiple decision trees to create an ensemble and improve classification accuracy. A typical RF model using majority voting from multiple trees is shown in Fig. 2.7.

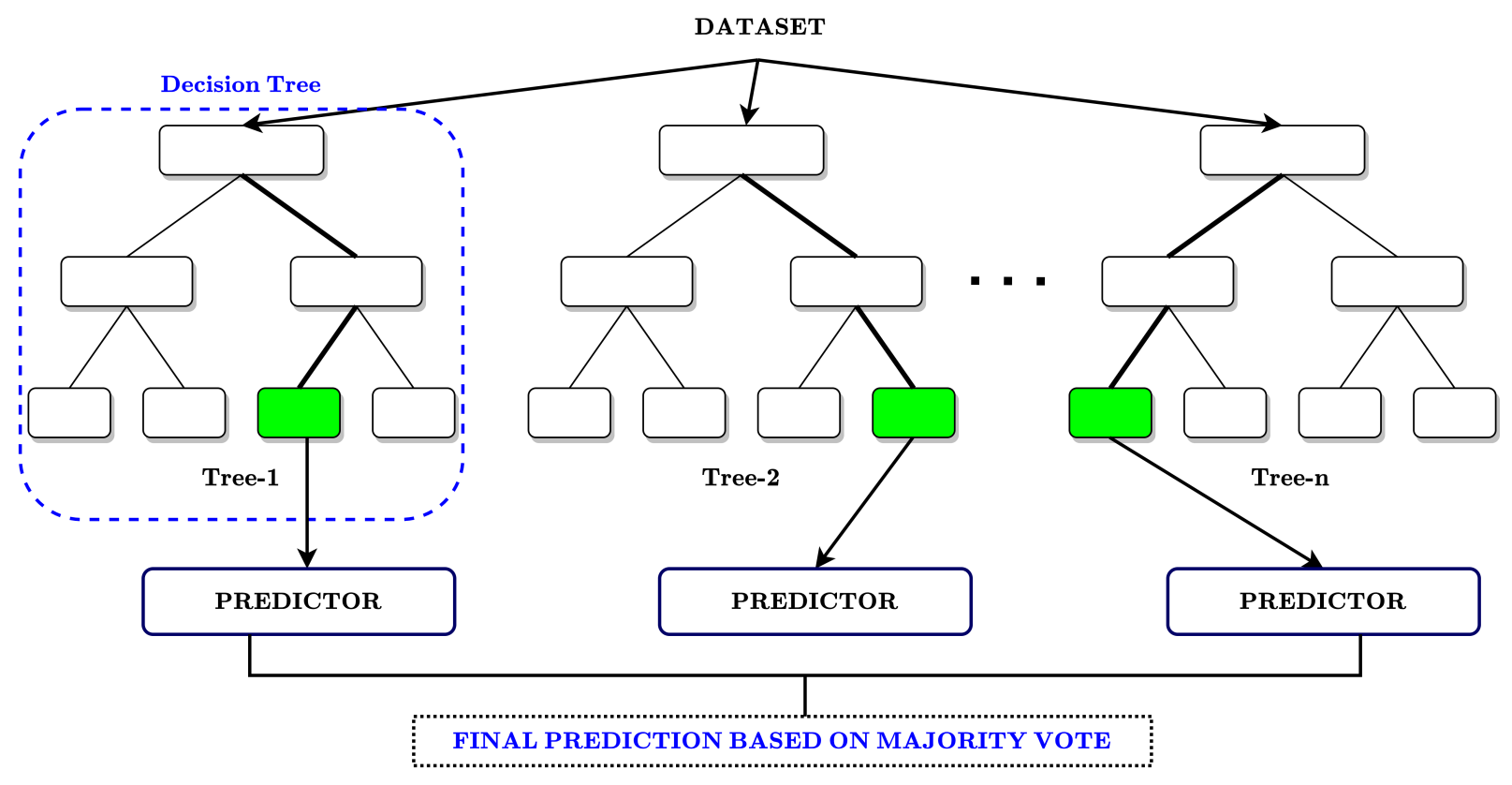

Figure 2.7: The framework of an RF model with majority voting.

\section{K-means clustering}

There are various unsupervised learning algorithms, and K-means clustering is popularly used in VDD. All the unsupervised algorithms employ various techniques to separate different classes by minimizing the distance between class points and maximizing the distance between two separate classes. A clustering algorithm is evaluated using a measure of goodness of fit. In the case of K-means clustering, the sum of squared errors (SSE) is defined as shown in the equation below to minimize the SSE.

$$
S S E=\sum_{i=1}^{k} \sum_{x_{j}}\left\|x_{j}-\mu_{i}\right\|^{2}
$$


ANN has shown promising results in modeling complex nonlinear datasets; however, they are prone to overfitting. [18] used a multi-stage ANN for damage diagnosis. The damage dataset was acquired using numerical simulation of a concrete bridge and a three-story frame. The structure was divided into small substructures, and ANN was applied independently to each sub-structure to improve damage detection and reduce the computational complexity of each ANN. [59] combined finite element methods and ANN with natural frequencies as features to identify the severity and localize damage in steel bridges. The proposed method was further improved the damage detection accuracy by incorporating an adaptive neuro-fuzzy system [58]. [41] used a data fusion by combining frequency response functions and ANN for member connectivity and mass changes in a two-story frame structure. ANN models were first trained individually and later combined through an ensemble for damage detection. The ensemble network showed improvement over individual ANNs. [44] employed sparse coding with ANN by majority voting to improve damage detection in numerical and experimental bridge structures.

SVM overcomes the problem of local minimization and inadequate statistical capabilities of ANN. For example, [48] used wavelet packet decomposition to extract feature vectors from acceleration responses as an input to SVM. The authors introduced the thin-plate spline Littlewood-Paley wavelet kernel function and showed superior accuracy with the kernel function over other conventional kernels for SVM. [82] proposed a genetic algorithm with SVM for damage detection in bridges. Numerical simulation on a simply supported bridge was used for data acquisition and showed the proposed algorithm has superior accuracy compared to genetic algorithms with ANNs. [51] compared SVM with optimization 
algorithms by using features of the AR model and residual errors of statistical parameters. It was shown a combination of various optimization algorithms performed superior to conventional SVM. Ensemble methods like RF were used to improve the damage detection in structures. [152] fused energy features of acceleration signals decomposed using wavelet packet transformation to feed as input to the RF model. The proposed method showed better results than conventional SVM, RF, and SVM with fused features.

Likewise, clustering has shown significant promises in VDD due to the use of unlabeled data. However, the determination of the clustering parameters is a limitation of these methods. Classification using clustering separates the dataset into several clusters based on the internal structure of the data. [113] used K-means clustering to distinguish smalldamages of stiffness reduction from undamaged in the cable-stayed bridge. The clustering parameter and similarity measure were identified using the global silhouette index and Gowda-Diday distance. [6] improved conventional K-means to isolate the outliers and detect jack arch damage in the Sydney Harbour bridge. There are other two clustering techniques, namely, hierarchical clustering [154] and density-based clustering [80], which were also used for damage detection.

Overall, ML algorithms are trained on a wide variety of data, and the accuracy of the algorithms improves with a larger amount of data. The purpose of training is to optimize the error along the dataset's dimensions using optimization functions such as a loss function or objective function and obtain the best prediction results for test data. However, ML algorithms need features that are obtained from different signal or image processing methods and are fed into different classifiers. Depending on the application, a 
suitable choice of features and classifiers is essential to identify anomalies from the images. [145] reviewed various ML-based SHM algorithms for isolating structural damage to steel pipes from environmental factors. In particular, [49] presented a comprehensive review of intelligent computational tools available for damage detection and system identification, with a specific emphasis on composite structures. The challenges associated with static and dynamic measurement techniques were discussed, along with future directions of automated and improved decision-making methods for SHM. Overall, it can be concluded from the literature that ML methods rely heavily on feature extraction, followed by the application of suitable classifiers. These methods can manage small anomaly datasets but may not be adequate for full-scale civil structures such as buildings, bridges, dams, pipelines, and wind turbines where damage patterns are complex and irregular (Yao et al. 2014).

\subsection{Drawbacks of the Traditional VDD Methods}

- The time-domain VDD methods are easier to implement, however, they only provide information about the time characteristics of the systems, and they are prone to noise contamination and environmental factors, which offers challenges for large structures. Frequency domain methods provide information about a change in frequency. However, they are not suitable for damage localization. In addition, the frequency changes caused by damage is usually minimal and may be suppressed in the changes caused by environmental and operational conditions.

- Conventional TF methods suffer from a trade-off between time and frequency resolution and are not suitable for nonlinear and nonstationary vibration data of VDD. 
- A critical limitation of AR models for feature extraction is the determination of suitable model order for a given dataset. The statistical time-series models provide information on damage detection and localization; however, they do not provide information about the severity of the damage.

- ANNs are computationally expensive if a large number of neurons are added and are prone to overfitting. SVM can only separate two classes and perform poorly for noisy datasets with overlapping classes. The selection of kernel function is also critical to its performance and is one of the other limitations. The RF is sensitive to noise and outliers and is not easy to interpret, and often requires intensive hyper-parameter tuning for better results. K-means clustering requires the determination of similarity measures and parameters $\mathrm{K}$ and also proved to be computationally expensive.

\subsection{Modern Techniques in VDD}

The nonstationary component of vibration response resulting from natural hazards poses difficulty in analyzing using traditional VDD methods that are based on the stationarity assumption of vibration response and selection of model orders. Apart from the excitationinduced nonstationarity, inherent damages in the structure also cause frequency-dependent nonstationarity in the response. The damage identification becomes a significantly challenging task with both amplitude and frequency-dependent nonstationary response, which can be solved using the advanced TF methods. Likewise, basis-free methods solve problems associated with pattern recognition methods requiring model orders or parameters in the case of supervised and unsupervised machine learning methods. Moreover, unlike 
ML methods, deep learning-based AI methods provide an excellent solution for long-term autonomous infrastructure monitoring and VDD. A brief introduction to advanced TF methods based on wavelet transform (WT) and deep learning-based methods is provided in this section.

\subsubsection{Advanced TF methods}

\section{(a) Wavelet Transform}

WT provides the essence of traditional Fourier transform with adjustable window location and size and an improvement over fixed window-based STFT. WT is primarily categorized into two different classes: discrete wavelet transform and continuous wavelet transform (CWT). With an appropriate basis function, WT offers flexibility to achieve better time and frequency resolutions together. The CWT is a signal processing technique that is used in many VDD applications such as signal noise filtering, data compression, and pattern recognition [8]. The CWT separates mixed signals into their components as well as filters out noise, and it is given by:

$$
W_{f}(d, \tau)=\int_{\infty}^{-\infty} f(t) \frac{1}{\sqrt{d}} \psi^{*}\left(\frac{t-\tau}{d}\right) d t
$$

where $d$ and $\tau$ represent scale and translation of the mother wavelet, respectively. $d$ relates to frequency scale, where a larger value of $d$ relates to a low-frequency signal and smaller $d$ relates to a high-frequency signal. At a specific time where the signal's spectral component is similar in scale to a value $d$, the product between the wavelet and signal will be higher. 
The wavelet shifts along with the signal to locate the frequencies within the time domain. The basis function is called mother wavelet $\psi(t)$, where superscript $(*)$ denotes its complex conjugate. With the appropriate choice of $d$ and $\tau$, the CWT utilizes the shifted and scaled versions of $\psi$ and subsequently forms its inner product with $f(t)$. However, its performance depends on the choice of basis function (e.g. bases such as Morlet, Daubechies, etc.). For example, in Cauchy CWT (CCWT), $\psi(t)$ is defined as follows [109]:

$$
\psi(t)=\psi_{\beta, m}(t)=\left(\frac{i}{(\beta t+i)}\right)^{m+1}
$$

where $m$ is a non-dimensional positive parameter $(m>1)$ and adjusts the frequency resolution of the signal. $\beta$ is a positive parameter whose dimension is the inverse of the dimension of the variable $t$. To eliminate the limitations of fixed frequency resolution of basic TF method, CWT has been explored in VDD for time-varying systems.

Wavelet-based frequency response function [42] was developed to detect abrupt changes in a time-variant system. In [133], two signal processing steps were used where the signal was first processed through CWT followed by the generalized discrete Teager-Kaiser energy operator that localized and magnified mode shapes of a damaged structure. A recent method [25] applying joint approximate diagonalization of the power spectral density matrices also yielded the operational deflection shapes of the structure. This method was then compared with several dominant characteristic deflection shapes to create a damage index and identify the damage location. Recently, [126] developed a two-step process for identifying the location of damage where CCWT was used for modal identification, followed by damage locating vectors applied to the identified flexibility matrix. In [155], a least-square 
SVM and time-dependent AR model was proposed to identify structural parameters of time-varying systems. However, the CWT and CCWT need significant improvement for frequency resolution to identify the minor damage as it is relatively less sensitive to minor frequency change in the structures [109].

\section{(b) Empirical Mode Decomposition}

The nonlinearity and nonstationary nature of vibration data were first addressed by HilbertHuang transform (HHT) [66, 139], which is basis-free in nature. HHT is an integration of Empirical Mode Decomposition (EMD) and Hilbert transform, and it can decompose any complex signal into a finite number of intrinsic mode functions (IMFs). An IMF is

a function that satisfies two conditions: First, the number of extrema and the number of zero-crossings have to be either equal or differ at most by one in the whole data set, and second, at any point, the mean value of the envelope denoted by the local maxima and local minima is zero. The process of extracting an IMF is called sifting. The signal after the sifting process can be represented as,

$$
x_{i}(t)=\sum_{j=1}^{n} i_{j}(t)+r_{n}(t)
$$

where $i_{j}(t)(j=1,2,3, \ldots, n)$ represents the IMFs of the original signal $y(t)$ and $r_{n}(t)$ is a residue of $y(t)$. Theoretically, every IMF must have only one frequency component. However, sometimes a single IMF contains multiple frequency components, called mode mixing, which poses difficulties in VDD in separating undamaged and damaged frequencies of the structures. A detailed literature review of several variants of EMD and their applications 
to VDD can be found in [21].

\subsubsection{Deep Learning methods}

Although AI is a broad area of research covering various engineering disciplines, machine learning (ML) and deep learning (DL) techniques are the two most popular branches of AI that have been heavily explored in VDD and SHM. Unlike ML (as discussed in section 2.1.2 (b)) that requires manual selection of features, DL-based AI methods automatically extract features and eliminate the need for manual feature extraction. Therefore, DL can differentiate among a large number of classes, and this capability has been recently explored for damage evaluation in structures [20]. DL algorithms are based on vast sets of labeled data and require high computational performance and memory requirements. The term "deep" refers to the large number of layers that exist between the raw image input and the final classification output used in a network. Convolutional neural networks (CNNs), a popular class of DL methods, have been successfully used since their breakthrough in the 2012 ImageNet challenge due to their ability to automatically extract features. This has enabled automatic and optimized feature extraction to become part of the classifier learning process, which, however, does not compromise its optimality or the accuracy of crack identification and VDD, as illustrated in Fig. 2.8.

In the context of SHM, DL can be used for damage detection in three ways: (a) classification, i.e., labeling an image as damaged or undamaged, (b) localization, i.e., locating the regions where damage exists using bounding boxes and identifying their coordinates, (c) segmentation, i.e., segmenting the pixels of an image into damaged and undamaged 
pixels (e.g., labeling of all pixels). Several methods have been developed in the last few years, including, but not limited to, the audio signal, time-series, video, and natural language datasets. CNN is the most popular variant of the DL network. The underlying architecture of CNN is comprised of three layers: (a) convolutional (feature extraction), (b) pooling (dimensionality reduction), and (c) fully-connected layer. The convolutional layer contains a finite number of filters (defined by the kernel or filter size) that convolves with the input data and identify a large number of relevant features from the input image. The pooling layer reduces the dimensions of the resulting features using a down-sampling operation, thereby minimizing the overall computational effort of the network. Depending on the data and the desired accuracy, the system is deepened by repeating the convolutionpooling sequences multiple times. However, CNN has been mostly explored in image-based structural damage identification, with limited applications in VDD.

In VDD-based literature, DL methods such as CNN, Fully convolutional network (FCN), or Recurrent neural network (RNN) are used to identify, classify, and quantify the damage. [56] explored a sparse coding-based CNN algorithm with wireless sensors for efficient bridge SHM. Sparse coding was used as an unsupervised layer for unlabelled data to learn highlevel features from acceleration data. Various levels of damage cases were considered for a three-span bridge that was instrumented using wireless sensors. The proposed method was compared with other methods such as logistic regression and decision trees, and the proposed method was shown to outperform other methods with an accuracy of $98 \%$. 


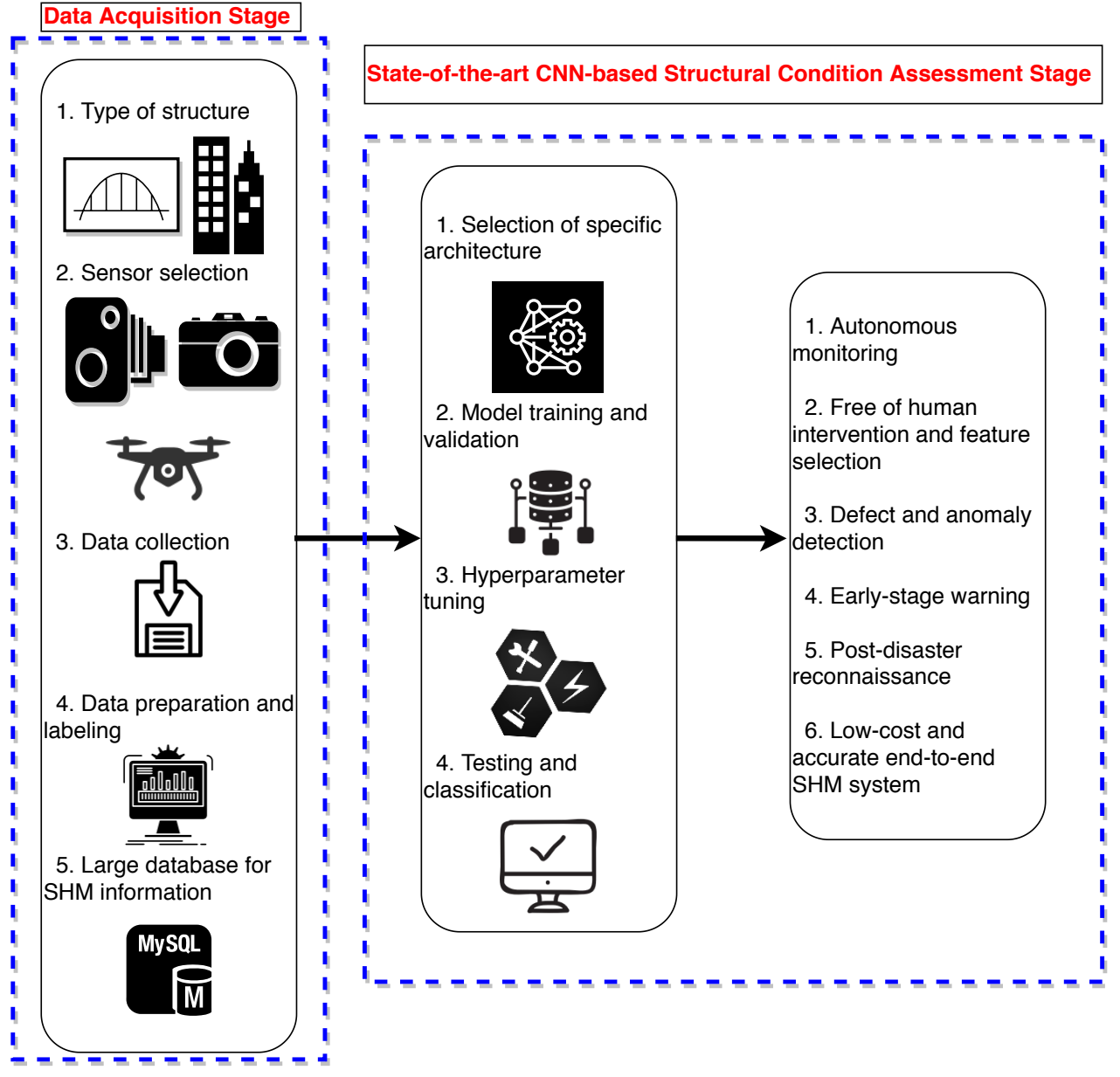

Figure 2.8: A schematic of the state-of-the-art CNN-based SHM operations [119].

[55] proposed a methodology for structural damage identification using CNN. Numerous undamaged and single-damaged samples of a steel gusset plate connection created in ABAQUS with varying uniformly distributed loads were developed to train, validate, and test the algorithm. Moreover, 50 network configurations with various hyper-parameters were tested over several epochs to determine the optimal CNN parameters. Recently, [98] proposed a 1D CNN-based technique in combination with autoencoder data compression for anomaly detection in a long-span suspension bridge. Similarly, [16] proposed a CNN- 
based VDD technique for damage identification in highly compressed data. A four-story numerical quarter-scale IASC-ASCE SHM model was used for numerical verification, and the proposed model was also validated on experimental studies using the IASC-ASCE SHM benchmark building and the Qatar University Grandstand Simulator. 1D CNN was also used in a further study by [149] to detect changes in stiffness and mass in three structural assemblages, a T-shaped steel beam, a short steel girder bridge, and a long steel girder bridge.

Primarily created for object recognition, 2D CNN algorithms are mostly explored for 2D images in various SHM applications to detect defects and anomalies autonomously. For vibration-based SHM, the researchers attempted to reshape the vibration signal into images by transforming the signal in the frequency and TF domain and used the resulting TF maps as the images in 2D CNN. However, the images involve significant complexity in choosing a large number of labeled data and layers and are not suitable for VDD of real structures. To alleviate this problem, 1D CNN [74] was recently introduced such that a time-history of vibration signal can be directly fed into CNN, which requires simple array operations, thereby demanding a shallow architecture with fewer hidden layers. However, multiclass VDD associated with a large-scale structure still remains a challenge.

\subsection{Challenges in the Modern VDD Methods}

Modern VDD methods overcome some of the limitations of conventional techniques. However, there is still a need to explore new methods that facilitate autonomous and costeffective infrastructure monitoring, which forms the gap areas of the modern VDD methods 
that are listed below.

- Discrete damage detection has been the main focus of the existing VDD methods. Progressive damage detection is still an unexplored area of damage detection and requires methods to evaluate and visualize progressive damage without userintervention for autonomous SHM. It is also crucial to thoroughly identify the efficacy of VDD on the sensitivity (i.e., severity level) and duration of the damage.

- Existing VDD methods require numerous measurements that are often unavailable due to the cost of sensors and installation and limited access to all critical locations. Although system identification methods have been explored using limited sensors, damage identification using a limited number of measurements has still not been fully explored. In this context, it is worth to study the sensitivity of the damage localization with respect to the number of limited sensors.

- Autonomous VDD requires creating a damage detection framework that can learn and assess the structural condition using minimal to no user intervention. DL methods, such as 1D CNN, have recently shown promising results in learning and creating an autonomous framework without requiring manual extraction of features. However, multiclass VDD of real-world structures is yet to be explored within the framework of 1D CNN.

- Feature-free VDD using limited training data and noisy measurements and the selection of suitable network architecture pose another challenge in 1D CNN. While 1D CNN captures relevant information in a batch of samples, it lacks the ability 
to learn the long-term dependencies of the sequential vibration data, which is relevant for VDD over a long period of data.

\subsection{Thesis Objectives}

Based on the above gap areas of the existing VDD literature, the key objectives of the thesis are as follows:

- Develop an algorithm to detect discrete and progressive damage in structures that is sensitive to minor level of damage (Chapter 3).

- Develop a basis-free method to identify the location of damage using limited sensors (Chapter 4).

- Investigate a feature-free DL technique to perform multiclass damage localization (Chapter 5).

- Develop an autonomous framework with an ability to learn from long-term dependencies of the sequential vibration data, solving real-world challenges of VDD using limited training data (Chapter 6).

\subsection{Summary}

In this chapter, a brief overview of the traditional and modern VDD is presented, along with their challenges and limitations. Traditional methods have been used for many decades because of their simplicity and ease of interpretation of results. Modern methods are now 
capable of providing effective damage detection schemes using next-generation sensors such as drones, smartphones and passing vehicles. However, they still lack the capability to prevent user intervention and the use of limited sensors and limited datasets for damage detection and localization. The basis-free methods and deep learning-based methods are proposed to overcome these shortcomings. The proposed methods are capable of providing an autonomous framework for multiclass VDD using limited sensors and training data. 


\section{Chapter 3}

\section{Damage Detection using}

\section{Synchrosqueezing transform-based}

\section{MEMD}

The objective of this chapter is to evaluate advanced time-frequency techniques to identify and visualize discrete and progressive damage in structures. A sensitivity analysis is also presented to study the efficacy of the proposed method on sensitivity and duration of damage. A hybrid method using synchrosqueezing tranform (SST) integrated with Multivariate Empirical Mode Decomposition (MEMD) is proposed for tracking the time-varying

behavior of structures using multi-sensor measurements. It is shown that the proposed method is capable to improve, detect, and visualize both discrete and progressive damage in structures using a suite of numerical, experimental, and full-scale studies. 


\subsection{Introduction}

Identification of time-varying systems such as structural damage detection is one of the essential elements of SHM and is critical for confirming the satisfactory performance of civil structures. In a structural system, the damage is initiated due to changes in material and geometric properties or boundary conditions of the system. As these properties change with time, the structure is mathematically modeled as a time-varying dynamical system. Damage could be either discrete due to an unwanted shock or progressive due to frequent accumulation of instantaneous damage that can lead to sudden catastrophic failure. Existing damage identification techniques involve analysis in time-domain, frequency-domain, as well as in the time-frequency domain [21] along with various artificial intelligence techniques [111].

The most common TF method is wavelet transform (WT), where a signal is decomposed into a series of local basis functions [96]. WT examines local data with a "zoom lens and an adjustable focus" to provide multiple levels of details and approximations of the original signal. In [65], structural damage detection was undertaken by detecting spikes in the wavelet coefficients. [72] provided an excellent summary of damage detection using wavelet with its applications in crack detection. A recent study [109] explored the use of Continuous Cauchy Wavelet Transform (CCWT) combined with tensor decomposition to understand progressive damage in the structures. The authors explored the capability of CCWT to delineate modal parameters. However, the study revealed that the frequency resolution needs significant improvement for CCWT to identify the severity of damage as it is sensitive to frequency change in the structure. In this chapter of the thesis, a powerful 
variant of WT, Synchrosqueezing Transform (SST), is explored to improve the sensitivity of identification of time-varying parameters of structures.

SST is a particular case of a reallocation method where the frequency is reassigned to achieve a sharpened TF representation of the signal [40],[138], [132]. SST has found its application in detecting, decomposing, and representing complex nonstationary timevarying signals such as seismic signals, hurricanes, and gravitational waves [61], [131]. SST combines the localization and sparsity properties of TF representation with the invertibility of TF transform. Unlike standard TF methods such as STFT or CWT that does not take advantage of sparsity in the signal, SST applies a nonlinear post-processing mapping to a conventional STFT or CWT representation. The mapping is designed to squeeze the energy in STFT closer to its most prominent frequencies, resulting in a sparse and concentrated TF representation of the signal [14]. The damage characteristics of any structures with respect to its modal frequency lie in its sensitivity to change with any change in the structural property. The study [76] explored various TF techniques for damage detection by showing the change in the natural frequency of the structure. It was shown that SST outperforms other techniques in the representation of the frequency shifts as the frequency component is squeezed and localized over time.

In [93], damping identification was carried out using SST. It was shown that SST was unable to separate closely-spaced frequencies. In another article [80], SST was used for modal identification of high-rise structures. The study by [102] proposed an improved methodology for identifying natural frequencies and damping ratios using random decrement technique and SST. The proposed method was compared with complete ensemble 
EMD and STFT. [112] presented a novel idea of detecting, locating, and quantifying the damage severity in the high-rise structures. The authors used SST to eliminate noise from the signals and nonlinear-dynamics based fractality dimension for identifying and locating the damage in the structures. A damage index was proposed for presenting the severity of the damage. The representation of the SST highly depends on the wavelet parameters, and signal reconstruction becomes challenging in some cases, which was overcome using an analytical mode decomposition [135]. It was concluded that the closely spaced frequencies pose a challenge for general SST. [83] proposed a multi-combination method for low-frequency structures by introducing analytical mode decomposition in combination with a recursive Hilbert transform and SST.

In a recent study [88], the applicability of SST to underdetermined systems with unknown source number was explored in combination with methods such as density peak clustering (DPC) and $l_{1}$-norm decomposition. The proposed method employed SST for sparse TF representation by coefficient reassignment along frequency followed by identifying normalized TF vector using a single source point method. In the end, DPC was used to identify the source number from the signals obtained from a machine. However, source identification using DPC could be inaccurate in the presence of noise, which can introduce inaccuracy in modal parameters. In another study [92], a combination of SST with unsupervised learning technique, $k$-nearest neighbors was used for modal identification of civil structures. However, the authors did not explore time-varying behavior and damage detection using SST.

In this chapter, an improved SST integrated with Multivariate Empirical Mode Decom- 
position (MEMD) is proposed for tracking the time-varying behavior of structures using multi-sensor measurements. MEMD has not yet been explored for damage detection. On the other hand, the performance of SST is known for its enhanced frequency resolution, while its incapability to decompose the closely spaced modes using multichannel measurements is improved using MEMD. While doing so, the capability of both methods is improved. The chapter is outlined as follows. First, in Section 3.2, a background of SST and MEMD is presented, followed by the description of the proposed algorithm in Section 3.3. Numerical simulation, experimental studies and full-scale study are conducted next in Sections 3.4, 3.5, and 3.6, respectively, followed by the summary of the proposed research in Section 3.7.

\subsection{Background}

A brief background of the SST and MEMD method is presented first before moving into the details of the proposed algorithm.

\subsubsection{Synchro-squeezing Transform (SST)}

SST is a time-frequency (TF) method that can characterize a signal with time-varying oscillatory properties. It is designed to analyze and decompose signals into the following form:

$$
x(t)=\sum_{k=1}^{K} A_{k}(t) e^{2 i \pi \phi_{k}(t)}
$$

where $A_{k}$ and $\phi_{k}$ are time-varying amplitude and phase functions respectively. 
SST is a variant of TF reassignment, a class of techniques that apply a non-linear postprocessing mapping of CWT. In case of synchro-squeezing, one reallocates the coefficients resulting from a CWT to achieve a concentrated TF picture, from which instantaneous frequency can be extracted. The basic steps of extracting the instantaneous frequencies using SST originate with the CWT, $W_{\psi} x(a, t)$ at a scale $a$ and time shift $t$ and is given by,

$$
W_{\psi} x(a, t)=a^{-1 / 2} \int_{\infty}^{-\infty} x(u) \psi\left(\frac{\overline{u-t}}{a}\right)
$$

The phase transform $\omega_{x(a, t)}$, is defined as the derivative of the complex phase of $W_{\psi} f$,

$$
\omega_{f(a, t)}=\frac{\frac{d}{d t} W_{\psi} x(a, t)}{2 \pi i W_{\psi} x(a, t)}
$$

Intuitively, this nonlinear operator can be thought of removing the influence of $\psi$ from the CWT and "encoding" the required localized frequency information using,

$$
S_{x(t, \eta)}=\int_{\left\{(a, t): \eta=\omega_{x(a, t)}\right\}} a^{-3 / 2} W_{\psi} x(a, t) d a
$$

The instantaneous frequencies are then extracted using,

$$
\omega(a, t)=\frac{-i}{W_{s}(a, b)} \frac{d}{d t} W_{s}(a, b)
$$




\section{(a) Frequency resolution of SST}

An example of a sine signal $x(t)=\sin (16 \pi \mathrm{t})$ is adopted to demonstrate the performance of SST, where the time-history of the signal is shown, followed by CWT and SST as shown in Fig. 3.1. It is evident that CWT has a wider frequency spread around $8 \mathrm{~Hz}$ while SST improves the frequency localization by reducing smearing around the frequency component.

(a)

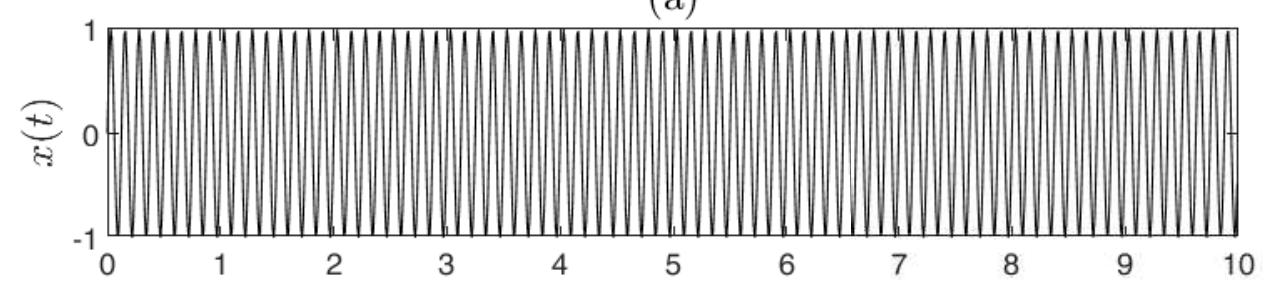

(b)

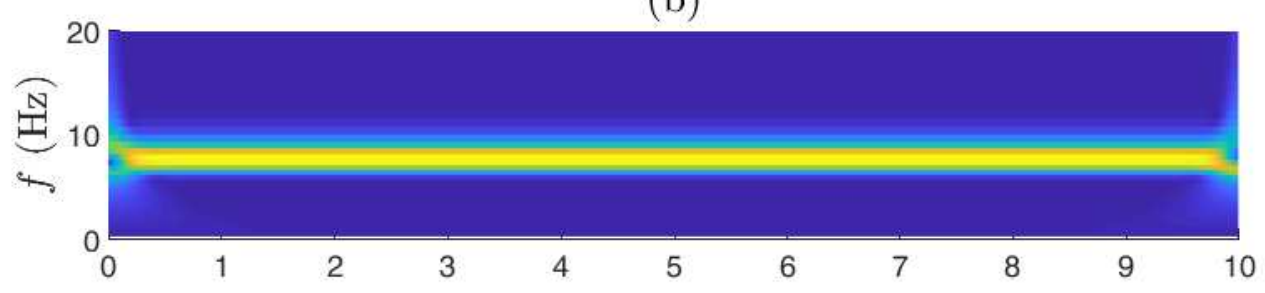

(c)

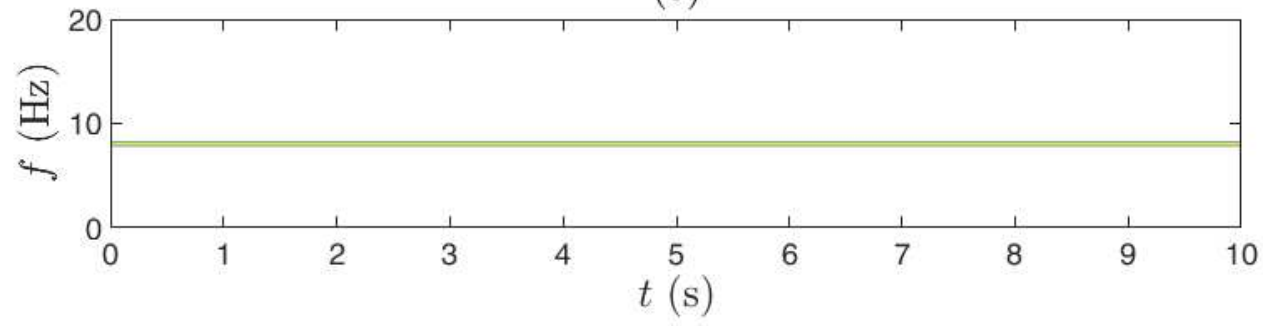

Figure 3.1: (a) The harmonic signal $x(t)$ and its (b) CWT, and (c) SST.

\section{(b) Signal representation using SST}

A mixture of sine signals containing five closely-spaced frequencies $(0.5 \mathrm{~Hz}, 0.7 \mathrm{~Hz}, 0.9 \mathrm{~Hz}$, $1.7 \mathrm{~Hz}$, and $7 \mathrm{~Hz}$ ) is used to illustrate the signal decomposition performance of SST. Figs. 3.2(a), 3.2(b), and 3.2(c) show the time-history of the signal, SST and Fourier spectra of 
the reconstructed signals, respectively. It is evident that SST is incapable of accurately separating the closely-spaced frequencies. For example, frequencies corresponding to 0.5 $\mathrm{Hz}, 0.7 \mathrm{~Hz}$ and $0.9 \mathrm{~Hz}$ are not clearly separated as shown in Fig. 3.2(c). In this chapter, the incapability of SST to decompose closely-spaced frequencies and to use multichannel measurements is improved using MEMD.

(a)

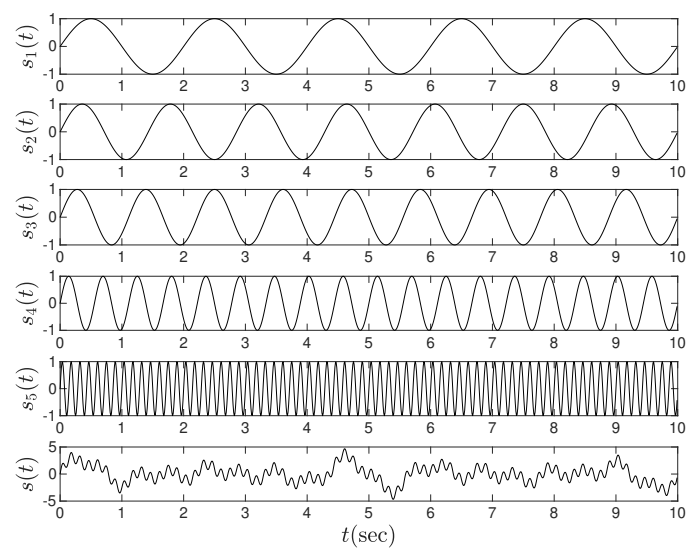

(b)

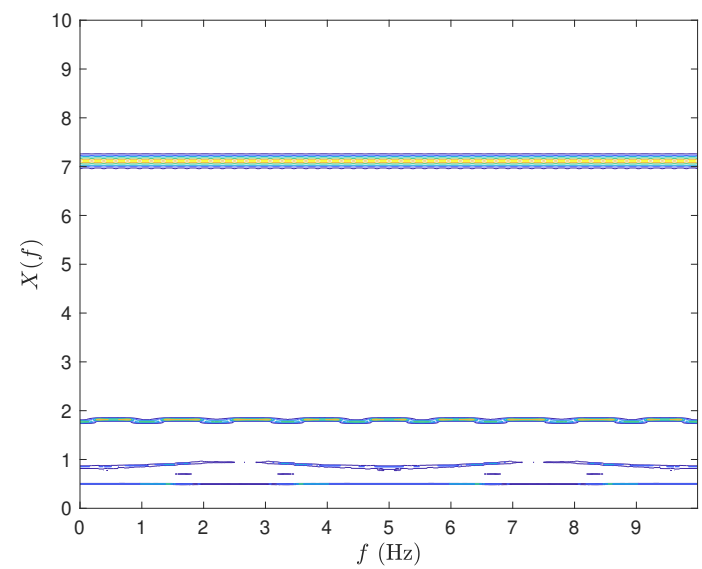

(c)

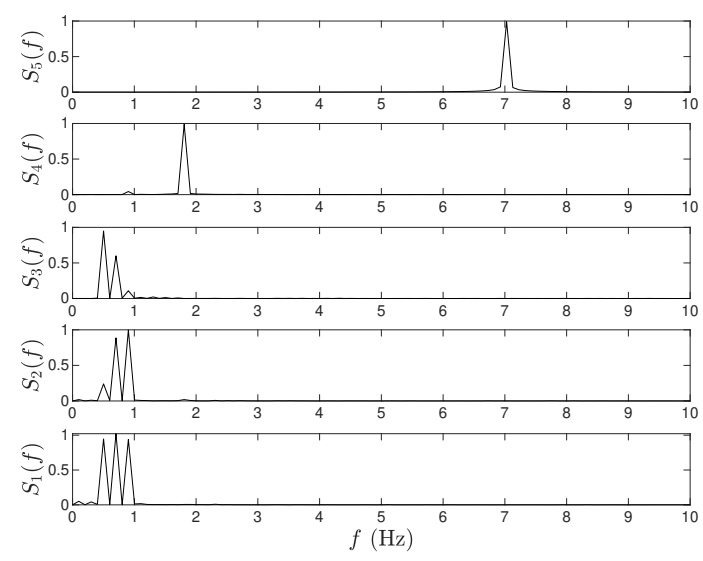

Figure 3.2: Mixture of the sine signals: (a) time-histories of the signals (b) SST representation (c) Fourier spectra of the reconstructed signal. 


\section{(c) Time-varying representation of frequency using SST}

One critical component of improved SST method over CWT is its capability to represent the TF information with better frequency resolution. The representation capability of SST is now evaluated by extracting its response for discrete and progressive change in frequency through simple examples, before pursuing SST in structural responses.

\section{Tracking of discrete change in frequency}

Sinusoidal signals are used to observe discrete and progressive change in the frequencies using CCWT and SST. A sine signal with discrete change of frequency from $10 \mathrm{~Hz}$ to $8 \mathrm{~Hz}$ is used to compare the performance of CCWT and SST. In Fig. 3.3, it can be observed that SST provides a clear indication of frequency shift at 5th second without affecting the frequency resolution. On the other hand, CCWT shows poor performance.

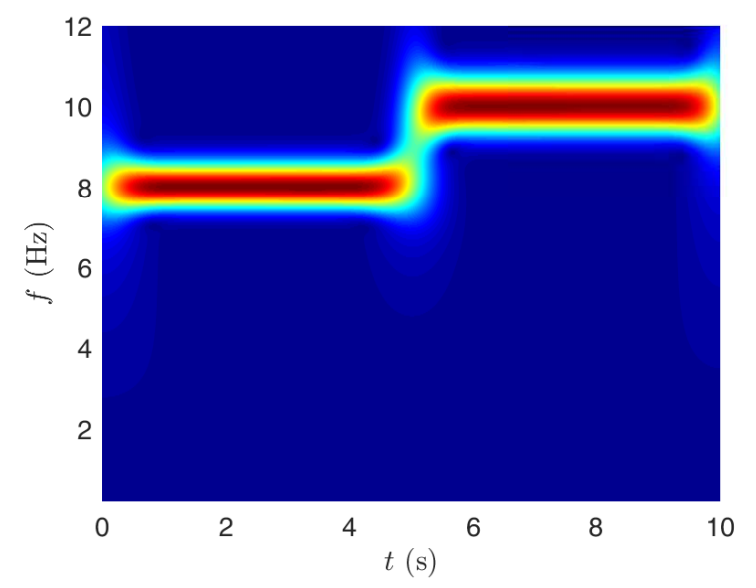

(a)

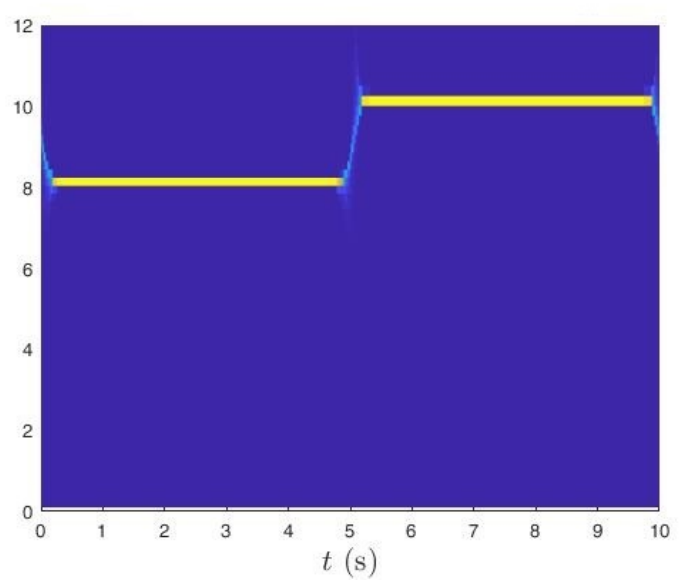

(b)

Figure 3.3: The discrete change in frequency: (a) CCWT and (b) SST of the signal. 


\section{Tracking of progressive change in frequency}

A chirp signal is used to study the capability of CCWT and SST in tracking the progressive change in frequency from $3.5 \mathrm{~Hz}$ to $7 \mathrm{~Hz}$. As shown in Fig. 3.4, SST leads to improved resolution as compared to CCWT and is also capable of tracking progressive change in frequency.

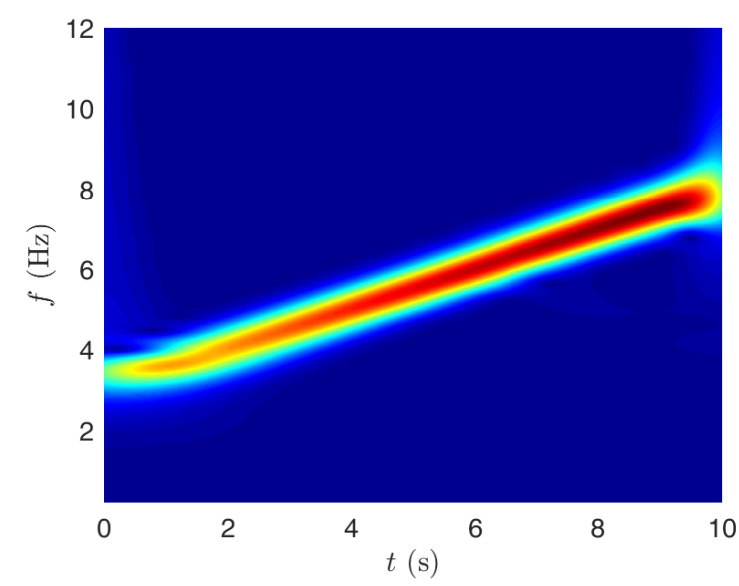

(a)

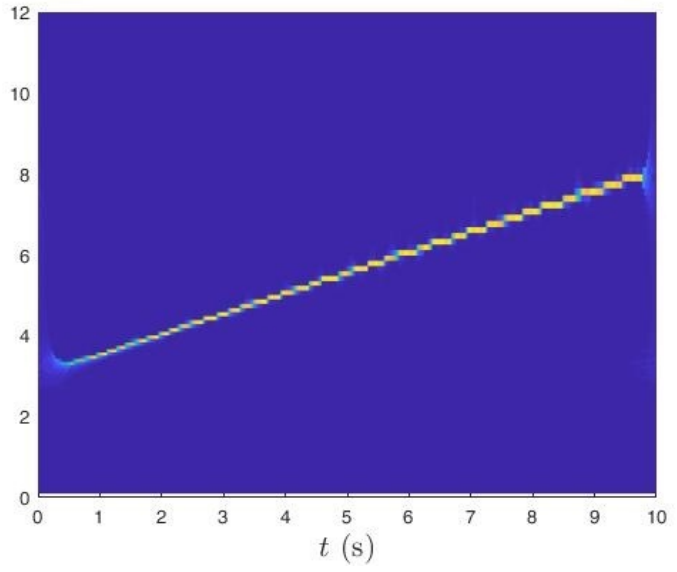

(b)

Figure 3.4: The progressive change in frequency: (a) CCWT and (b) SST of the signal.

\subsubsection{Multivariate EMD}

Vibration-based monitoring of real-life structures requires the use of multiple sensors to extract the structural modes that are often closely spaced. The incapability of SST to address these issues is overcome by using MEMD. For analyzing nonlinear and non-stationary signals, EMD [66] method works only for a single data measurement. For multichannel measurements, a multivariate extension is proposed by [105]. The basic idea of EMD is that any complicated data set can be decomposed into a finite number of 'intrinsic mode functions'. This decomposition method is adaptive and therefore, free of any basis func- 
tion. Since the decomposition is based on the local characteristic time-scale of the data, it is also applicable to nonlinear and nonstationary processes. In order to calculate local mean for multivariate signal, real-valued projections along multiple directions on $n$-spheres are used for envelopes.

The standard EMD method works only for a single real-valued signal. While dealing with the measurements of multiple sensors, EMD algorithm faces two problems [105]:

- Because of the single channel dependency of EMD, there is no guarantee that the decomposition of IMFs from different channels of measurements will match, either in number or their frequency contents.

- The joint information between multiple sensors is not realized because signals from multiple sensors are treated individually.

The MEMD method works with $n$-variate signal as multidimensional time series and maps them into suitable directions corresponding to multi-dimensional space. To estimate the uniform distribution groups in the MEMD, quasi-Monte Carlo lower deviation sequence is used to create the point groups on unit $(n-1)$ sphere. A suitable set of direction vectors are sampled on unit hyperspheres (n-spheres) based on both uniform angular sampling methods and quasi-Monte Carlo-based low-discrepancy sequences. In order to estimate all multi-dimensional envelopes, the multiple signals are projected onto the chosen direction vectors and the average of all envelopes is considered as a local mean of multiple signals. For $n$-dimensional signal $y(t)=\left[y_{1}(t), y_{2}(t), y_{3}(t), \cdots, y_{n}(t)\right]$ and the direction vectors over the $k$-th directions $D^{k}=\left[d_{1}^{k}, d_{2}^{k}, \cdots, d_{n}^{k}\right]$, the algorithm for MEMD is presented in Algorithm 1 (Rehman et al. 2010): 


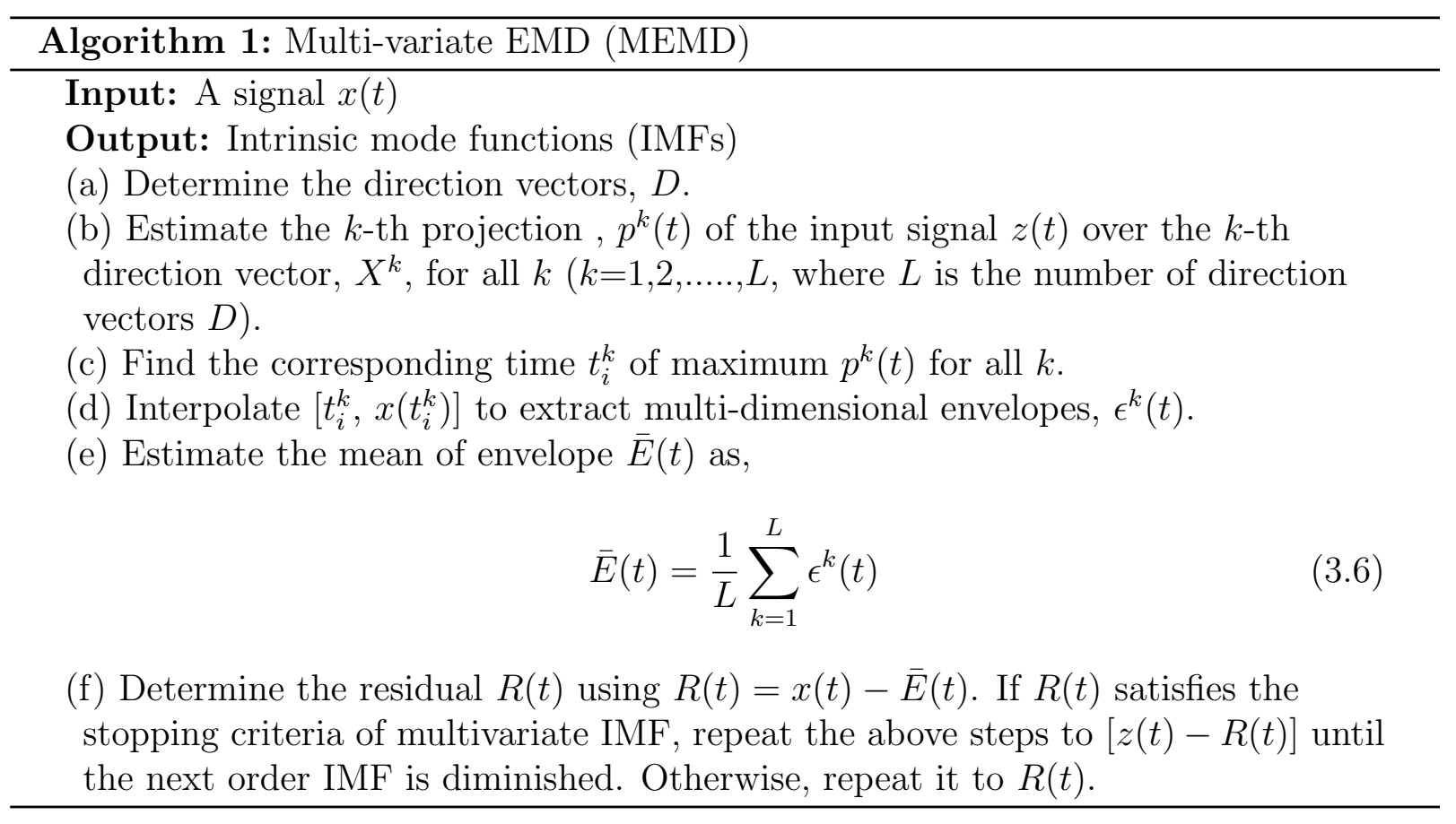

A simple example is used to illustrate the application of MEMD using a mixture of three harmonic signals, where, $f=\{1,3,5\} \mathrm{Hz}$ and is shown in Eq. 3.7. In the resulting mixture noise with signal-to-noise ratio of 10 is also added to mimic field conditions.

$$
X=\left[\begin{array}{ccc}
4 & -2 & 1 \\
-2 & 5 & -3 \\
1 & -3 & 6
\end{array}\right]\left[\begin{array}{c}
\sin \left(f_{1} t\right) \\
\sin \left(f_{2} t\right) \\
\sin \left(f_{3} t\right)
\end{array}\right]
$$

Three mixtures are analyzed using the MEMD and the resulting IMFs of each mixture $X_{i}(t)$ for $i=1,2,3$ are shown in Fig. 3.5. For example, first three rows of each column show the IMFs (i.e., harmonic signals) of each response. It may be observed that the MEMD results in mode-mixing in few IMFs due to the presence of measurement noise in the mixture. 

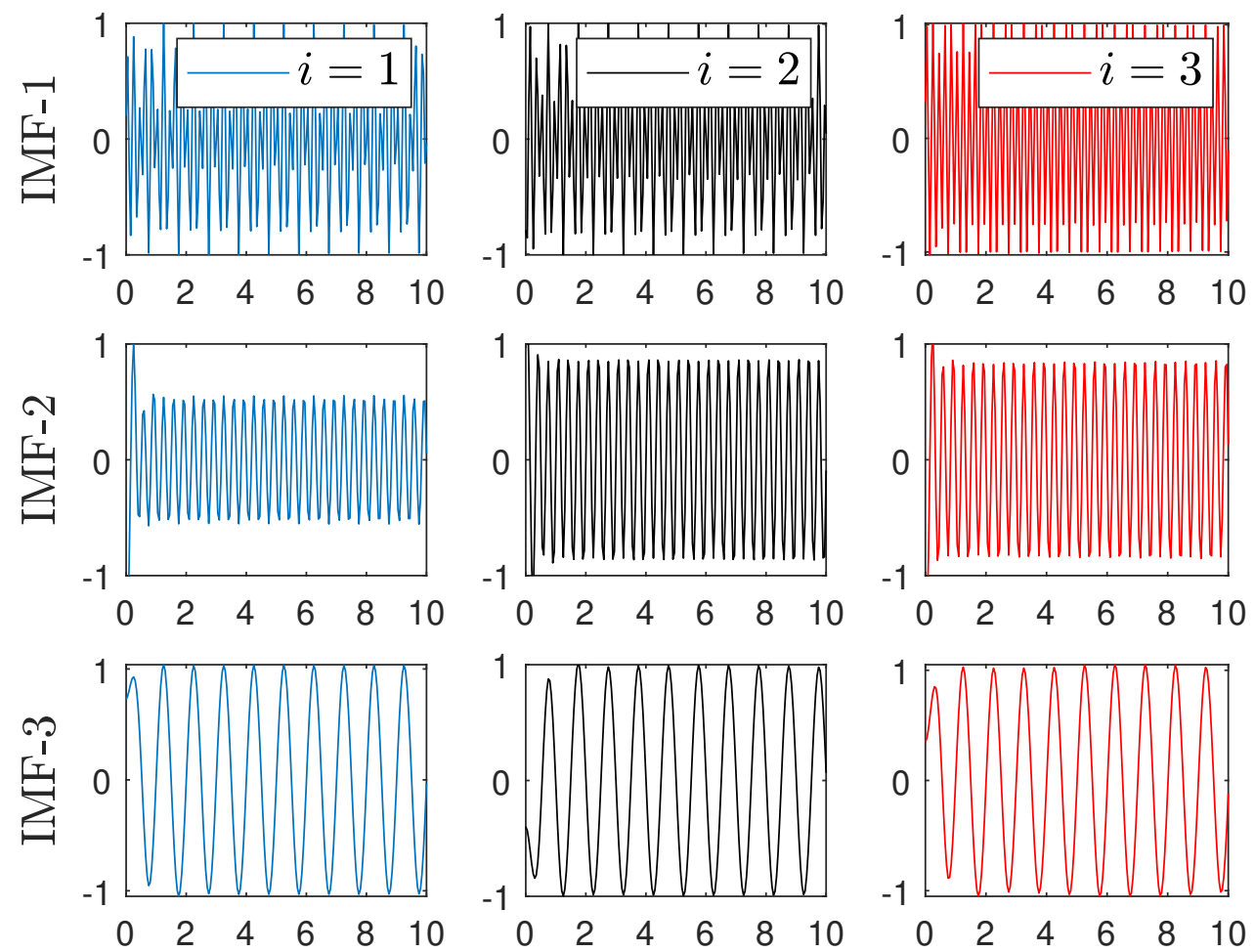

Figure 3.5: Illustration of the mode decomposition using MEMD.

\subsection{Proposed Algorithm}

Consider a linear dynamical system with $n$ degree-of-freedom (DOF) subjected to a broadband excitation $\mathrm{E}(t)$, the equation of motion is expressed as,

$$
\mathbf{M} \ddot{\mathbf{x}}(t)+\mathbf{C} \dot{\mathbf{x}}(t)+\mathbf{K} \mathbf{x}(t)=\mathbf{E}(t)
$$

where, $\mathbf{x}(t)$ is the displacement vector. Using the classical modal superposition theorem, The solution to Eq. 4.1 for those of broad-band $\mathbf{E}(t)$ can be written in terms of an expansion of vibration modes: 


$$
\mathbf{x}=\boldsymbol{\Phi} \nu
$$

where, $\mathbf{x}$ and $\boldsymbol{\nu}$ is the response and modal coordinate matrix, respectively. $\boldsymbol{\Phi}_{m \times n}$ is the modal transformation matrix. $n$ and $m$ is the number of modal responses and measurements, respectively. The measurement at the $i$-th $\operatorname{DOF}(i=1,2, \ldots, m)$ can be expressed as,

$$
x_{i}(t)=\sum_{j=1}^{n} \phi_{i j} \nu_{j}(t)
$$

where, $\nu_{j}$ is the $j$-th modal response and $\phi_{i j}$ represents the modeshape ordinate of $i$-th DOF and $j$-th mode.

Since the MEMD results in IMFs containing mono-component modal responses, each signal of $\mathbf{x}(\mathbf{t})$ can be expressed in terms of its IMFs:

$$
x_{i}(t)=\sum_{j=1}^{n} I_{i j}(t)
$$

where $I_{i j}$ is the $j$-th IMF of $x_{i}(t)$. Considering the similarity of Eq. 3.10 and Eq. 3.11, we get:

$$
I_{i j}(t)=\phi_{i j} \nu_{j}(t) \quad i=1,2, \ldots, m
$$

Each IMF, $I_{i j}$ is then evaluated to obtain SST as shown below, 


$$
S_{I(t, \eta)}=\int_{\{(a, t): \nu=\omega I(a, t)\}} a^{-3 / 2} W_{\phi} I_{i j}(a, t) d a
$$

The instantaneous frequencies are then extracted as,

$$
\omega(a, t)=\frac{-i}{W_{s}(a, b)} \frac{\partial}{\partial t} W_{s}(a, b)
$$

The proposed algorithm is presented in Algorithm 2.

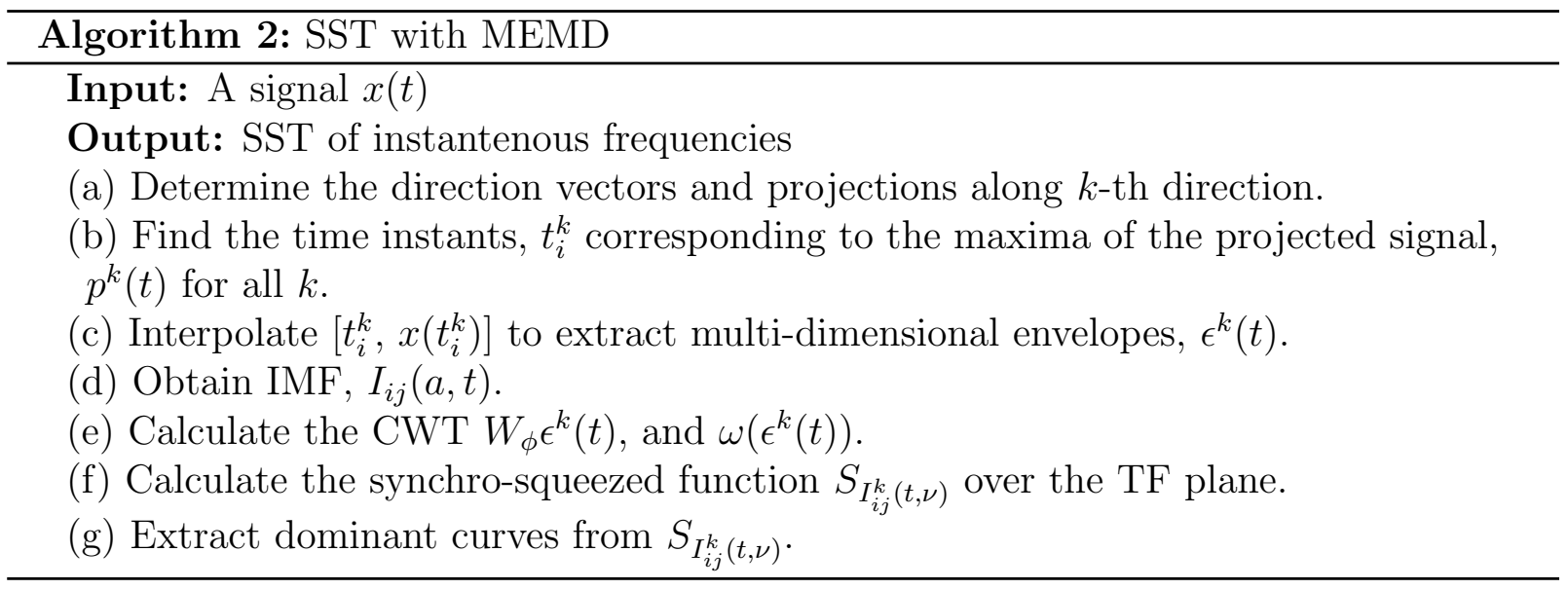

\subsection{Numerical Validation}

\subsubsection{SDOF model}

A single degree-of-freedom (SDOF) system as shown in Fig. 3.6 is selected to illustrate the performance of the proposed method. A $10 \mathrm{~kg}$ model with a progressive linear stiffness reduction from 5000 to $1000 \mathrm{~N} / \mathrm{m}$ between $40-80$ seconds is used for the illustration. Its performance evaluation is carried out by exciting the SDOF model with a harmonic load 


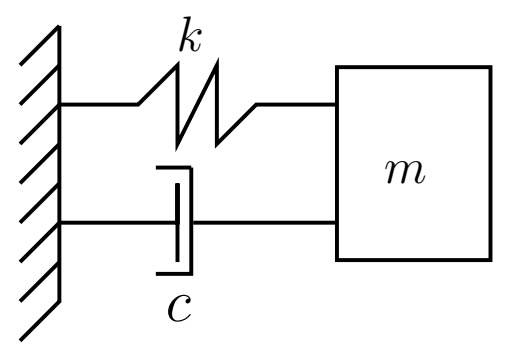

Figure 3.6: SDOF Model.

using a frequency of $5 \mathrm{~Hz}$. The SST is undertaken to the simulated acceleration response of the SDOF model under various severity and duration of linear reduction in the stiffness. Fig. 3.7 evaluates the frequency resolution $(\Delta f)$, i.e., the various extents of change in frequencies and Fig. 3.8 shows the various durations $(\Delta t)$ that are used to simulate such changes in frequencies starting with 5 seconds, 10 seconds, and 20 seconds, respectively. The results show the SST of the simulated responses (i.e., the instantaneous frequencies). The SST yields two inherent instantaneous frequencies of the data: one with structural frequency and another for the excitation harmonic. The SST presents sporadic use of data sets and does not take low entropy data samples into account, causing occasional shrinkage of data while converting from time domain to TF domain and entropy associated with the data. In this chapter, frequency change is used as an indicator to detect the time-varying properties of the structure. Before this indicator is used in the proposed method, these results show the capability of SST to detect the frequency change depending on the varying degree of frequency changes $(\Delta f)$ and durations of damage $(\Delta t)$. For example, Fig. 3.7(a) shows that SST is able to delineate the changes of time-varying frequency as low as 0.38 $\mathrm{Hz}$, indicating its capability to detect a minor change in structural frequencies even in case of damage lasting for a small duration. 
(a)

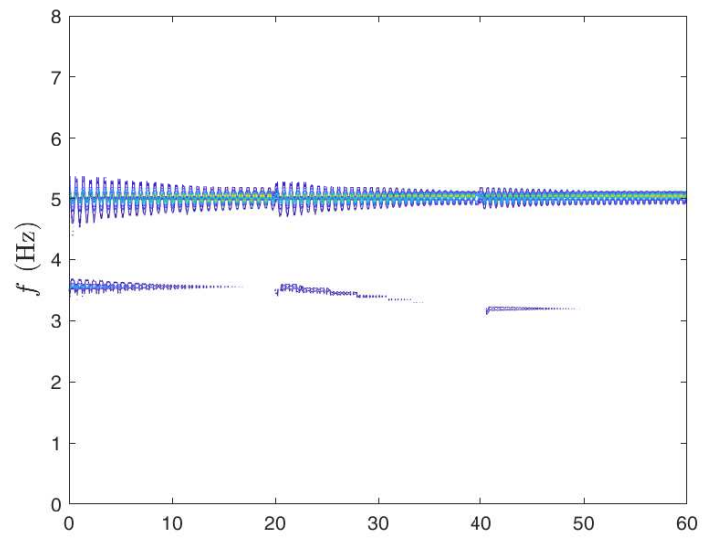

(c)

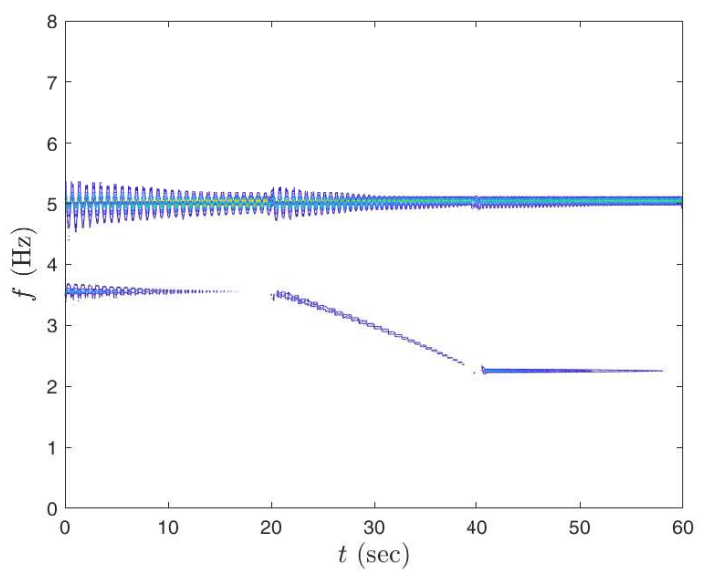

(b)

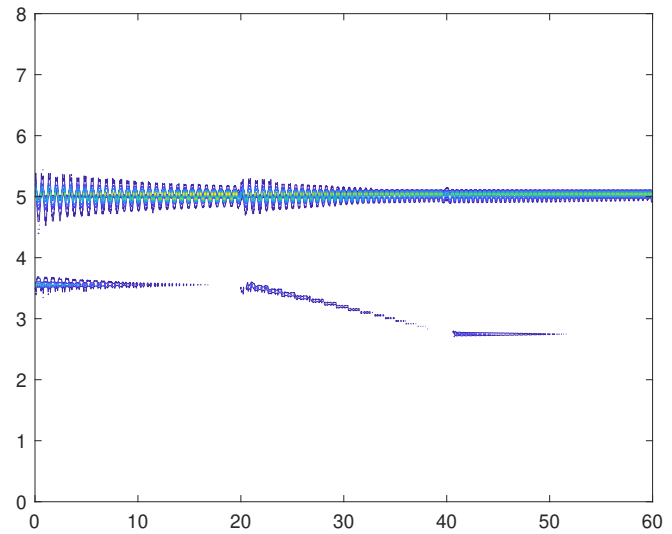

(d)

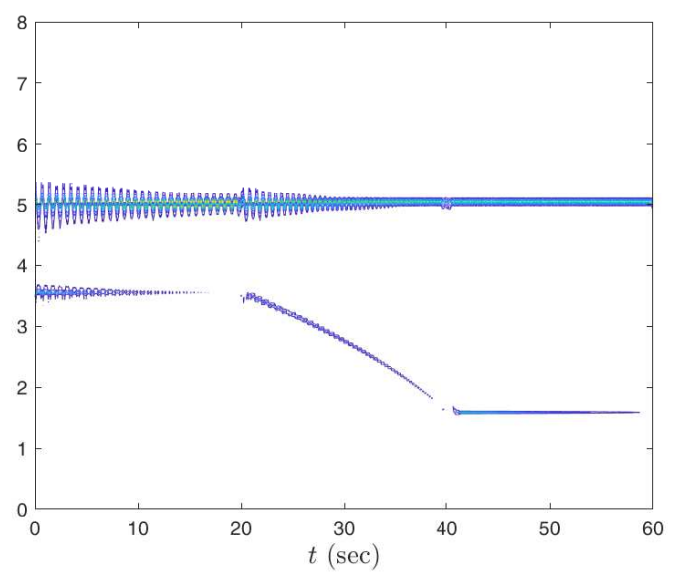

Figure 3.7: Effect of frequency resolution under harmonic excitation (a) $\Delta f=0.38 \mathrm{~Hz}$ (b) $\Delta f=0.80 \mathrm{~Hz}$ (c) $\Delta f=1.31 \mathrm{~Hz}$ (d) $\Delta f=1.97 \mathrm{~Hz}$, where $\Delta f$ represents the difference between undamaged and damaged frequency. 
(a)

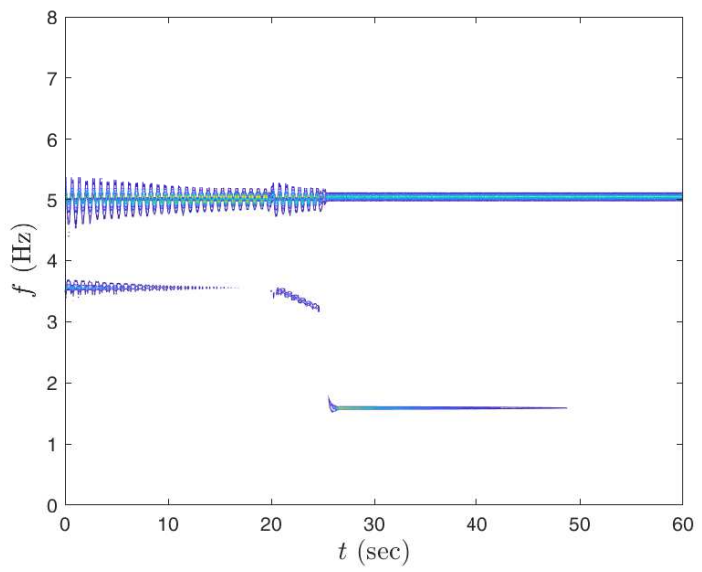

(b)

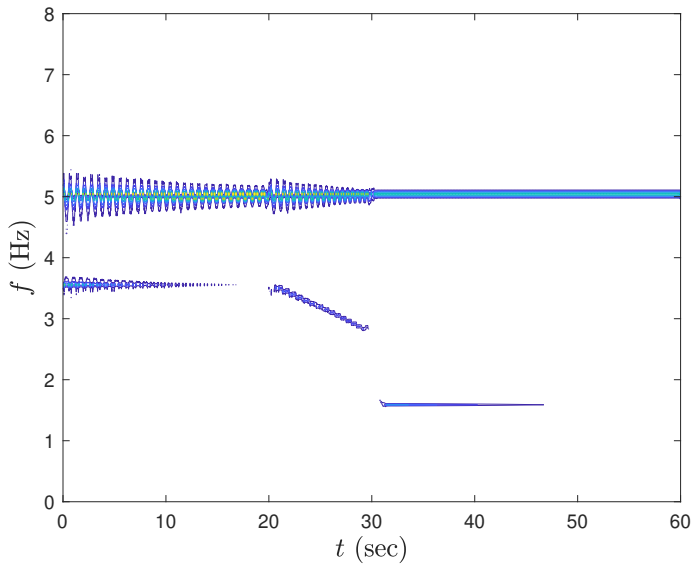

(c)

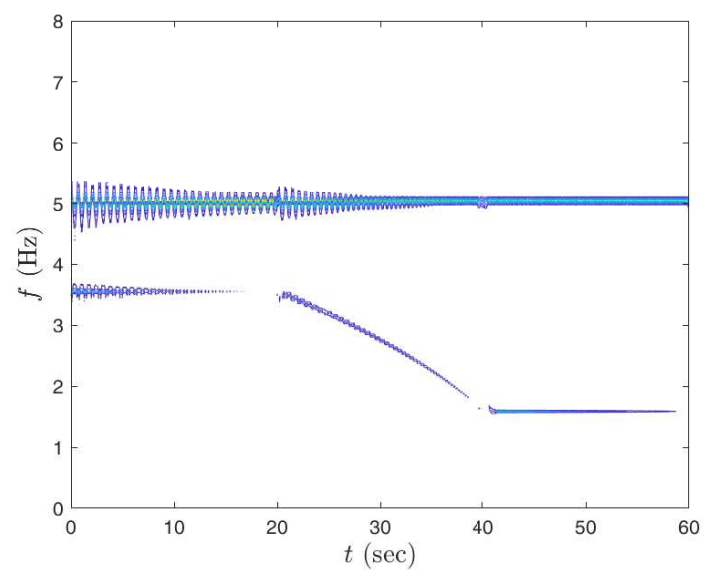

Figure 3.8: Effect of duration of damage under harmonic excitation (a) $\Delta t=5 \mathrm{sec}$, (b) $\Delta t=10 \mathrm{sec}$, (c) $\Delta t=20 \mathrm{sec}$, where $\Delta t$ represents the duration of linear reduction in the stiffness. 
(a)

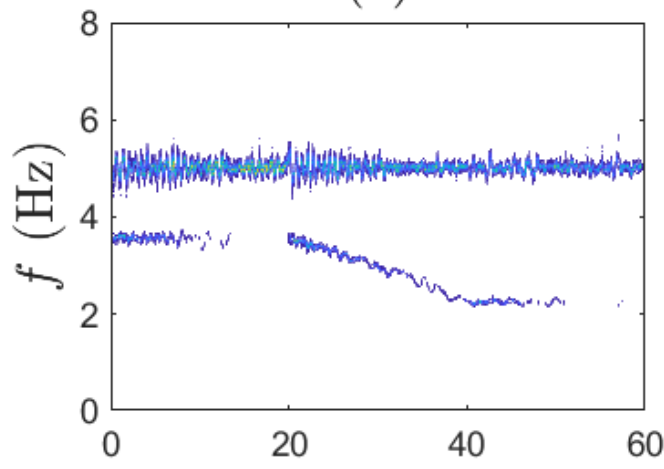

(c)

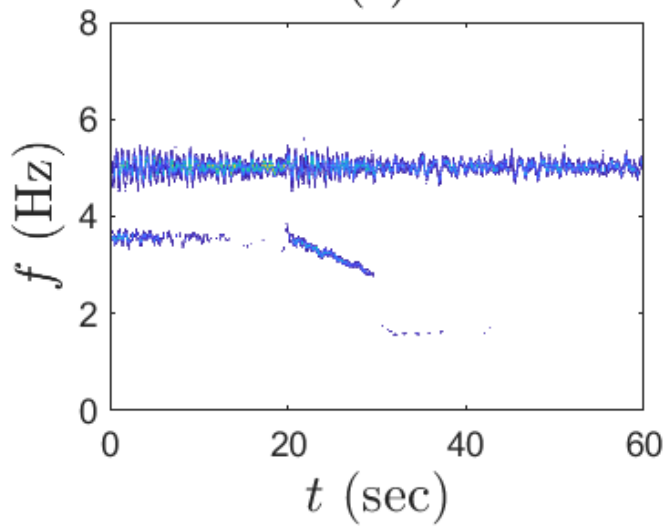

(b)

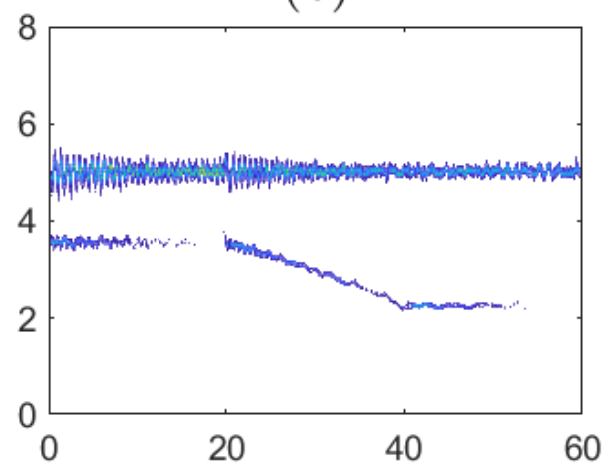

(d)

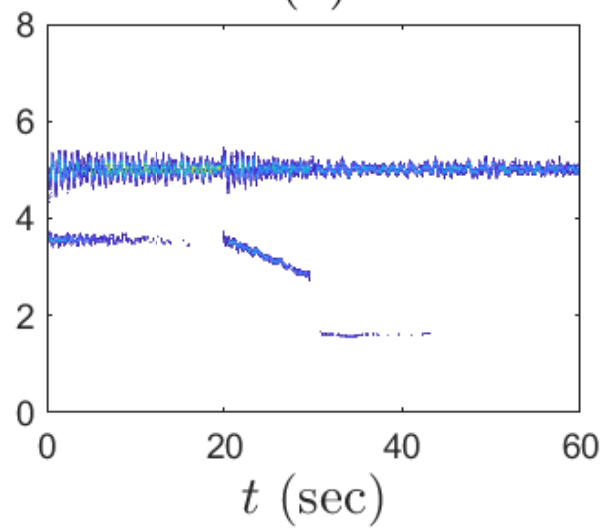

Figure 3.9: The simulated response with $\Delta f=1.31 \mathrm{~Hz}$, when there is a measurement noise of (a) $5 \%$, (b) $10 \%$, respectively; the simulated response with $\Delta t=10 \mathrm{sec}$, when there is a measurement noise of (c) 5\%, (d) 10\%, respectively.

The above simulated response is now contaminated with a varying level of measurement noise (i.e., $5 \%$ and $10 \%$ ) to show the robustness of the proposed method. First, the measurement noise is added to the simulated response when $\Delta f=1.31 \mathrm{~Hz}$ (i.e., the original signal corresponding to Fig. 3.7(c)). The resulting time-varying frequencies are shown in Figs. 3.9 (a) and (b) for the noisy signals corresponding to $5 \%$ and $10 \%$ measurement noise, respectively. The similar study is performed to the simulated response when $\Delta t=$ $10 \mathrm{sec}$ (i.e., the original signal corresponding to Fig. 3.8(b)). The resulting time-varying frequencies are shown in Figs. 3.9 (c) and (d) for the noisy signals corresponding to $5 \%$ and 
$10 \%$ measurement noise, respectively. It may be observed that SST has been successful to clearly delineate the change in frequency for a small duration even with a significant measurement noise, proving its adequacy to its signal representation capability under real situations.

\subsubsection{4-DOF model}

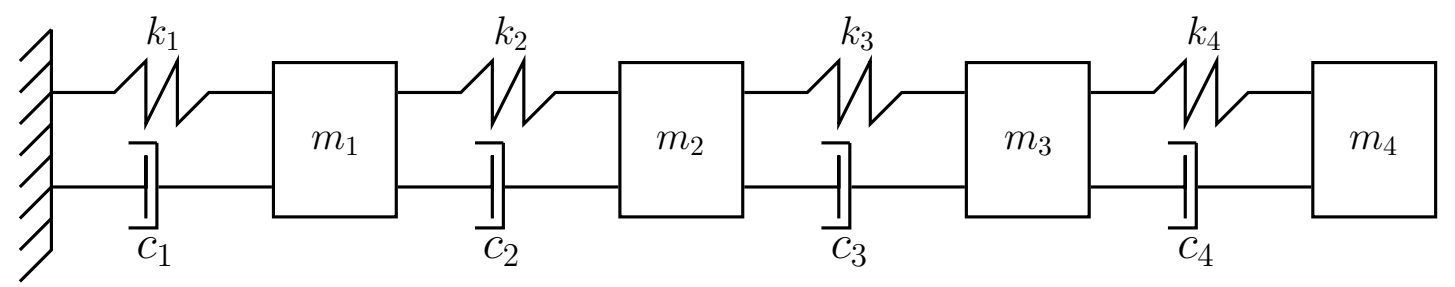

Where: $m_{1}=m_{2}=m_{3}=5.0 \mathrm{~kg}, m_{4}=10.0 \mathrm{~kg}$ and $k_{1}=k_{2}=k_{3}=k_{4}=200 \mathrm{~N} / \mathrm{m}$

Figure 3.10: 4-DOF Model.

In this section, a 4-DOF model is used as shown in Fig. 3.10 and the simulated acceleration measurements are used to illustrate the proposed method. A modal damping ratio of $3 \%$ is considered for all modes. Four different damage scenarios are considered to check the sensitivity and accuracy of the proposed method under realistic conditions. For example, Cases 1-3 represent 40\%, 30\% and 20\% damages in all the floors, respectively, whereas Case 4 represents $20 \%$ damage in the first floor and $60 \%$ damage in the second floor with no damage in the third and fourth floor. All the damages are simulated through a linearly stiffness reduction between 50th and 80th seconds such that the data contains both undamaged and damaged frequencies. The simulated responses are contaminated with $10 \%$ measurement noise. The frequencies of the undamaged and damage cases are shown in Table 3.1 . 
Table 3.1: Identified frequencies $(\mathrm{Hz})$ for the simulated damage cases.

\begin{tabular}{|c|c|c|c|c|c|}
\hline Mode \# & Undamaged & Case 1 & Case 2 & Case 3 & Case 4 \\
\hline \hline 1 & 0.29 & 0.22 & 0.24 & 0.26 & 0.23 \\
\hline 2 & 0.90 & 0.7 & 0.76 & 0.81 & 0.87 \\
\hline 3 & 1.47 & 1.14 & 1.23 & 1.32 & 1.19 \\
\hline 4 & 1.87 & 1.45 & 1.57 & 1.67 & 1.72 \\
\hline
\end{tabular}

Fourier spectra of IMFs obtained from MEMD for Case 2 and Case 3 are shown in Fig. 3.11 and Fig. 3.13, respectively. For example, Fig. 3.11 shows the FFT of the first four IMFs of the decomposed signal of Case 2 comprising of undamaged frequencies of $0.29 \mathrm{~Hz}$, $0.90 \mathrm{~Hz}, 1.47 \mathrm{~Hz}, 1.87 \mathrm{~Hz}$ and their corresponding damaged frequencies of $0.24 \mathrm{~Hz}, 0.76 \mathrm{~Hz}$, 1.23 Hz, and 1.57 Hz respectively. The SST for Cases 2-3 are shown in Fig. 3.12 and Fig. 3.14, respectively. It can be observed the higher modes are more scattered in comparison to the lower modes and lower modes have better resolution. Once the modal responses are extracted using the proposed method, auto-correlation function of modal responses is used to extract the modal damping ratio. Fig. 3.15 shows the estimation of modal damping ratio as obtained from the IMFs, which is closely matching with the theoretical value (i.e., $3 \%$ ). It may be noted that modal damping ratio is often sensitive to measurement noise and subjected to large estimation errors, when extracted from the vibration measurement (Brewick and Smyth 2014). Due to this reason, the modal damping ratio is not pursued for structural damage detection in this study. 

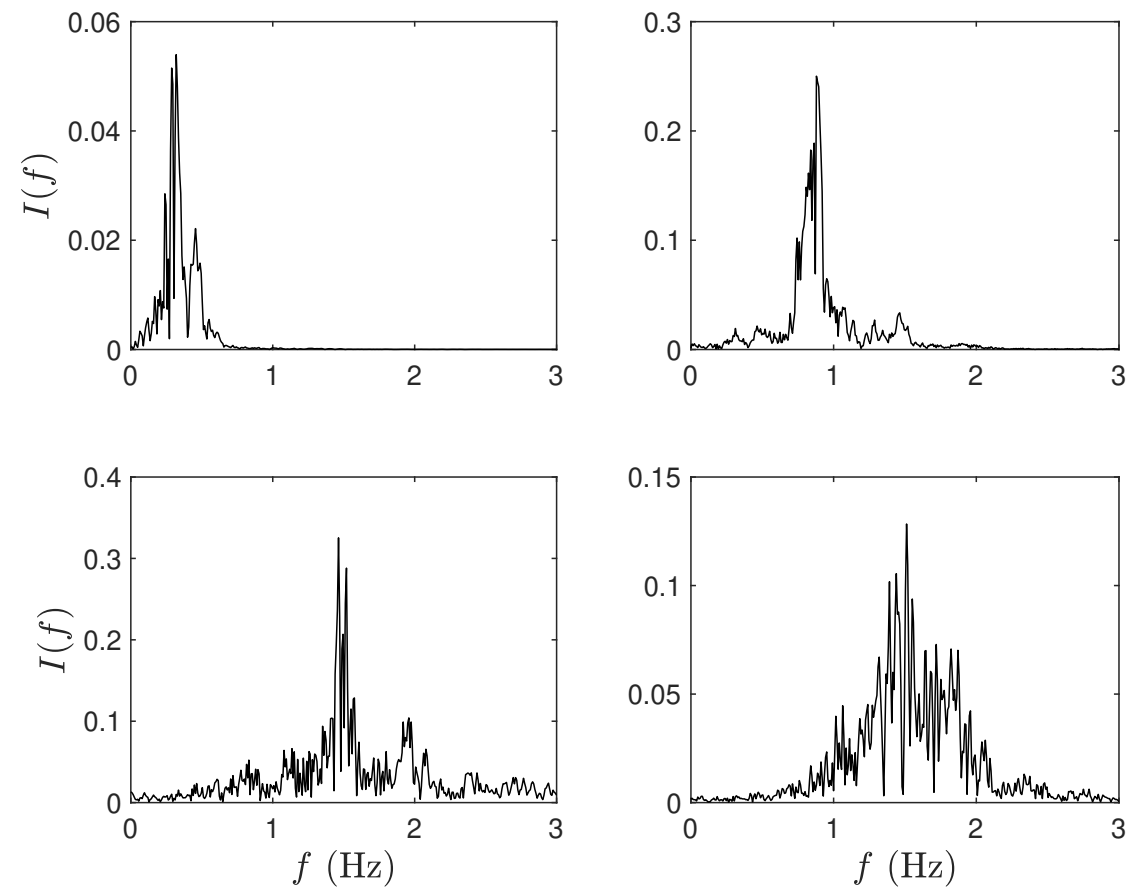

Figure 3.11: Case 2: Fourier spectra of the IMFs obtained from MEMD.
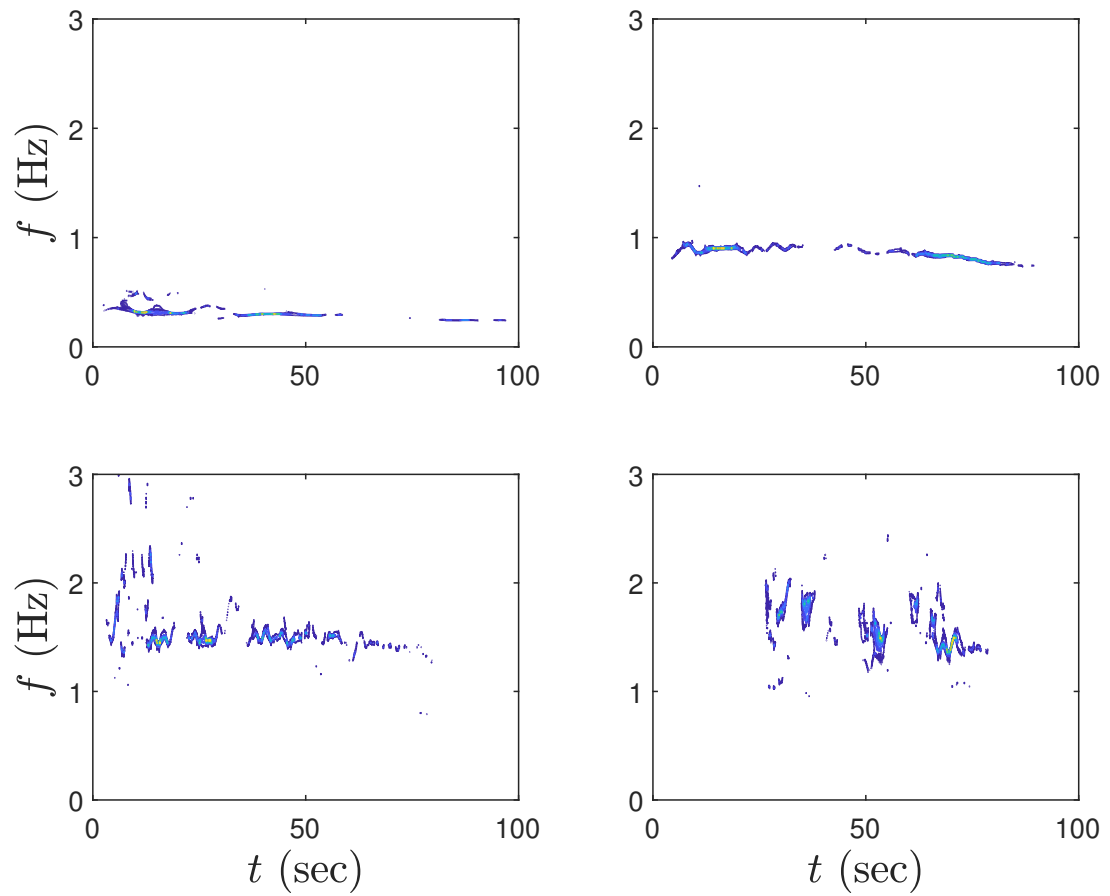

Figure 3.12: Case 2: SST of IMFs obtained from MEMD. 

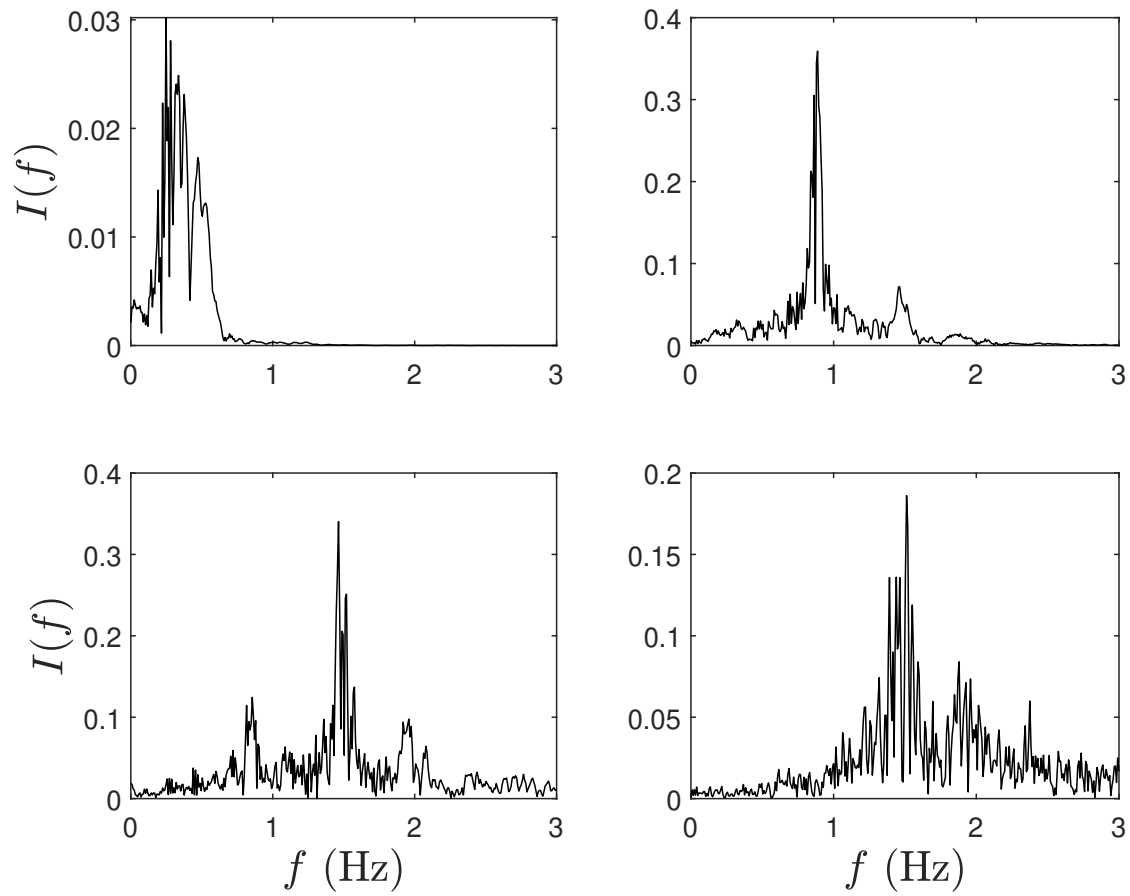

Figure 3.13: Case 3: Fourier spectra of IMFs obtained from MEMD.
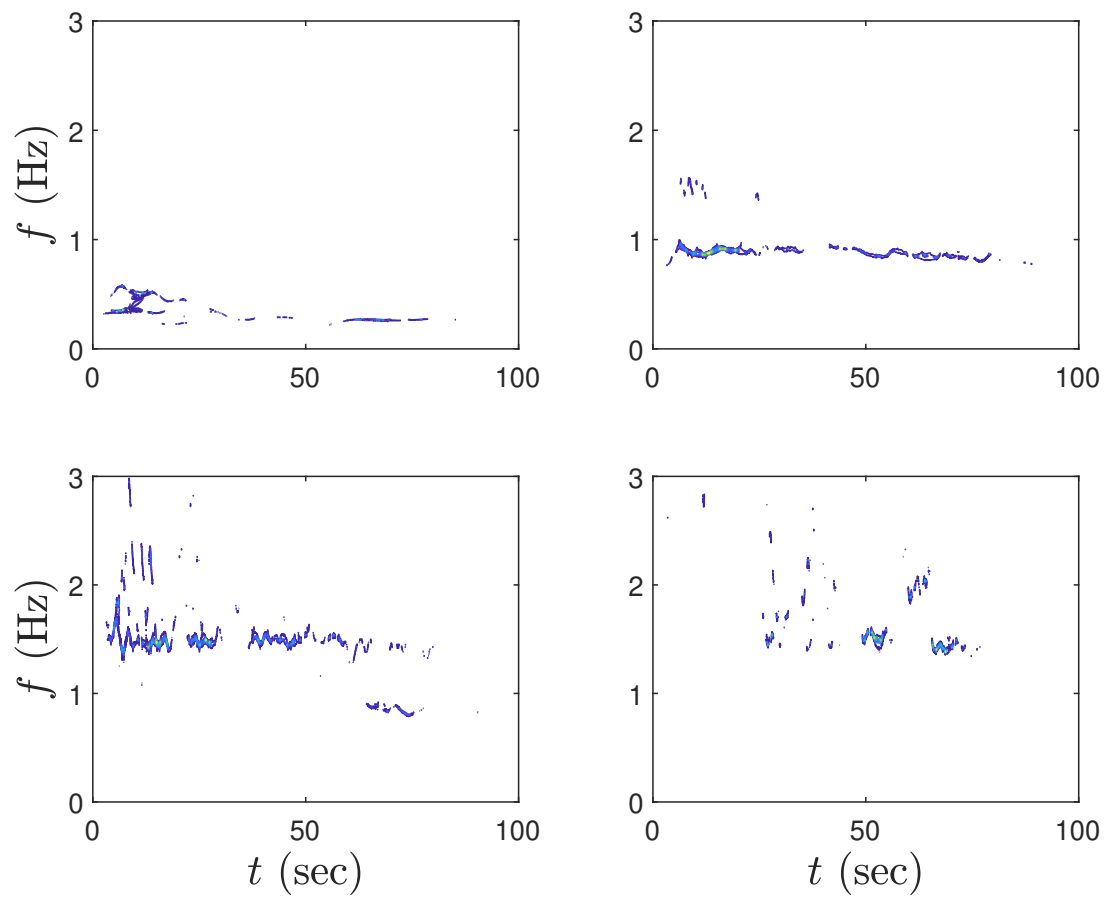

Figure 3.14: Case 3: SST of IMFs obtained from MEMD. 


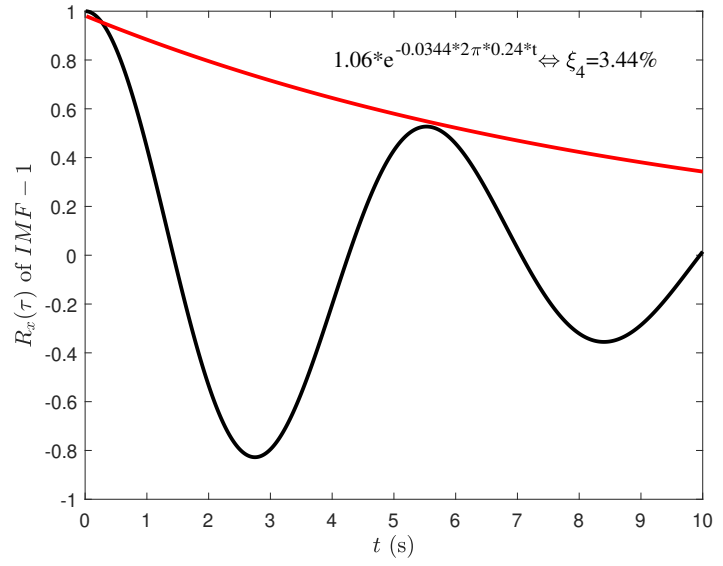

(a)

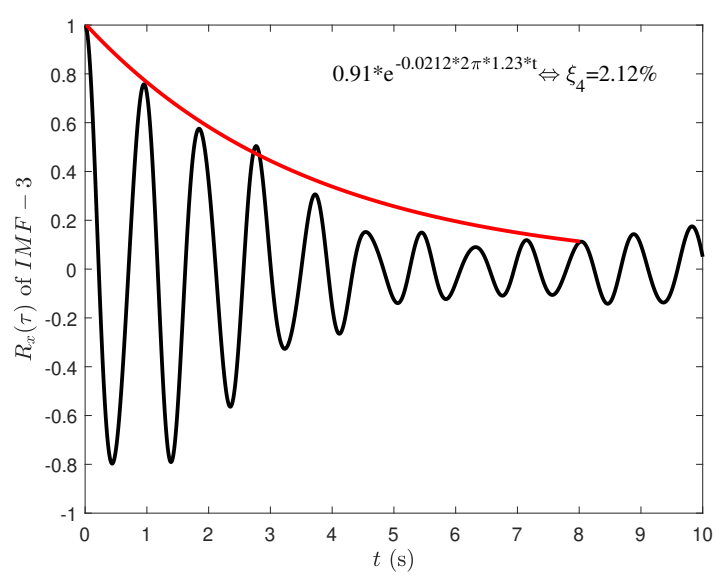

(c)

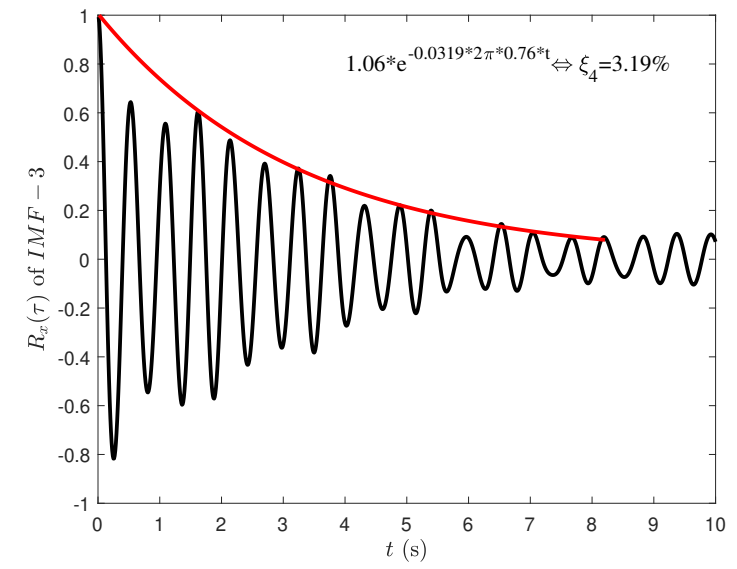

(b)

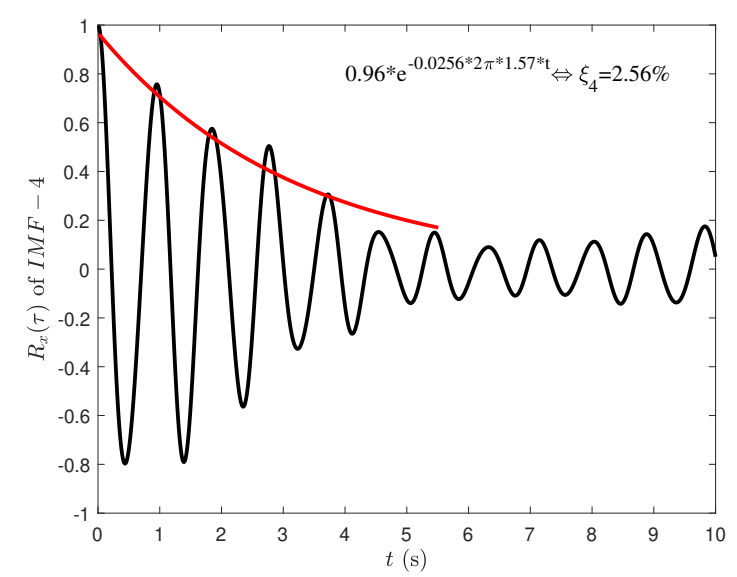

(d)

Figure 3.15: The estimated modal damping ratio of (a) first, (b) second, (c) third, and (d) fourth modes for Case 2.

\subsection{Experimental Validation}

In this section, a time-varying system [68] is utilized to illustrate the signal decomposition capability of SST. Fig. 3.16 shows the layout of the experimental setup that involves simulation of discrete and progressive damages, thereby the setup was perfectly suitable for the proposed research. The electromagnet's attachment and the rollers' position can 
be used as fixed parameters or as variable parameters to simulate damage at any time during testing. The drop mass experiment has an electromagnet that can be removed quickly to simulate a sudden change in mass and thereby introducing sudden change in natural frequency of the system. The moving roller experiment can be viewed as a mass changing over time, thereby creating a progressive change in the mass as well as its natural frequency. The specific details of this experiment can be found in [68] and are not repeated here.

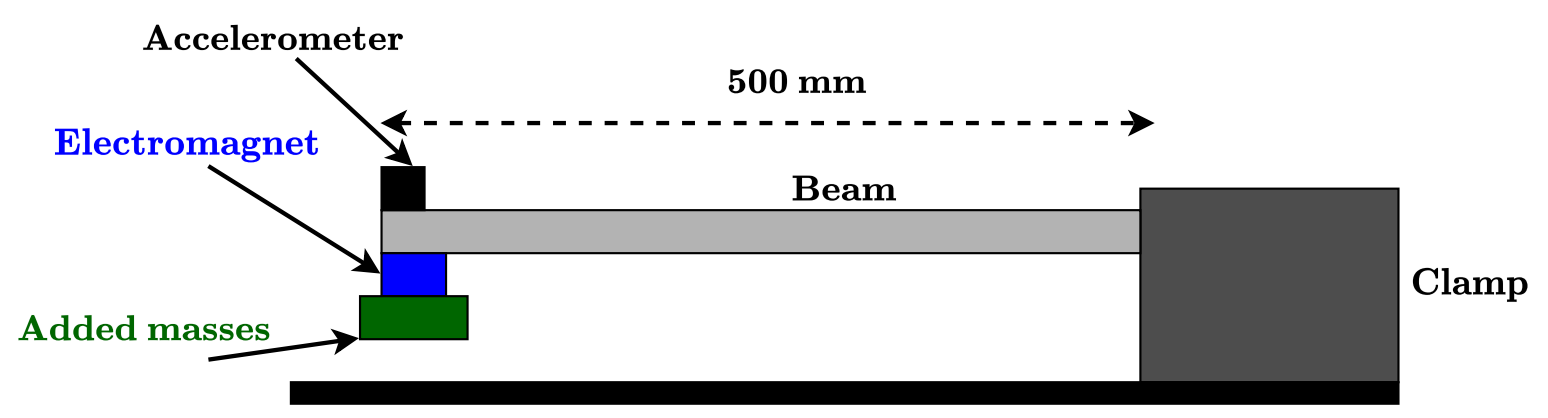

(a)

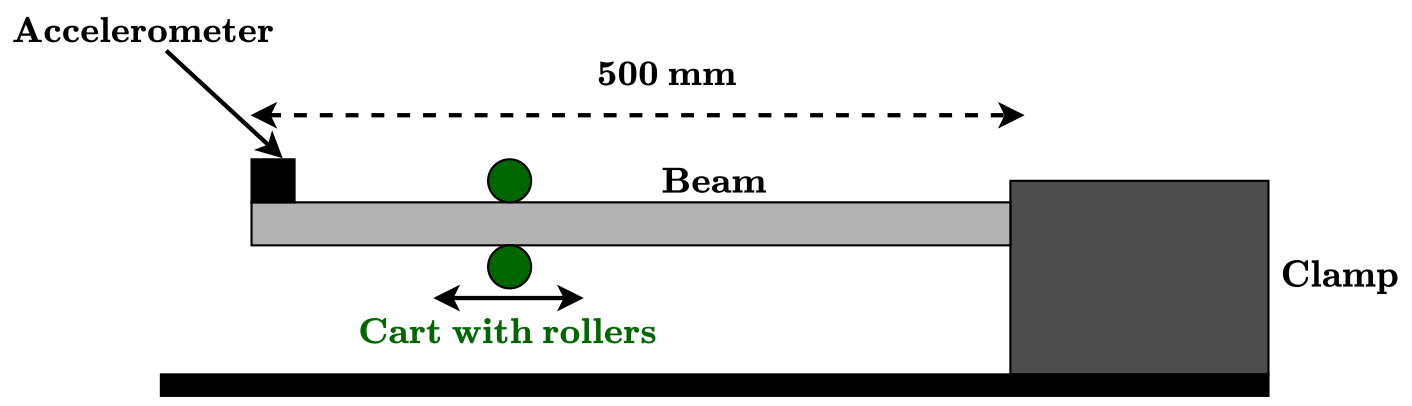

(b)

Figure 3.16: Schematic of experimental setup for damage simulation with detachable mass and roller that move along the beam. (a) drop mass setup, (b) moving roller setup.

SST of the acceleration time-history is presented in Fig. 3.17(a) and Fig. 3.17(b), respectively, showing discrete frequency change from $20 \mathrm{~Hz}$ to $12 \mathrm{~Hz}$ at ninth seconds for the mass drop experiment. The moving rollers create a stiffness changing mechanism, 
which progressively changes the natural frequency of the beam from 6.5 seconds to around 8 seconds and is shown in Figs. 3.18(a) and (b). The experiment setup (Joyce et al. 2018) is first struck by an impact hammer to run for some time (from 1 - 4 seconds), before the excitation is induced using the drop mass (Fig. 3.17) or moving roller (Fig. 3.18). Therefore, for the purpose of identification of time-varying frequency, the response starting from 4th second is considered for both the tests. In the moving roller test, the roller was returned to the original position at 9-9.5 seconds, followed by the termination of the experiment. Due to this reason, there is a sudden reduction in the frequency in the SST plot of Fig. 3.18(b), followed by a constant value of the instantaneous frequency.

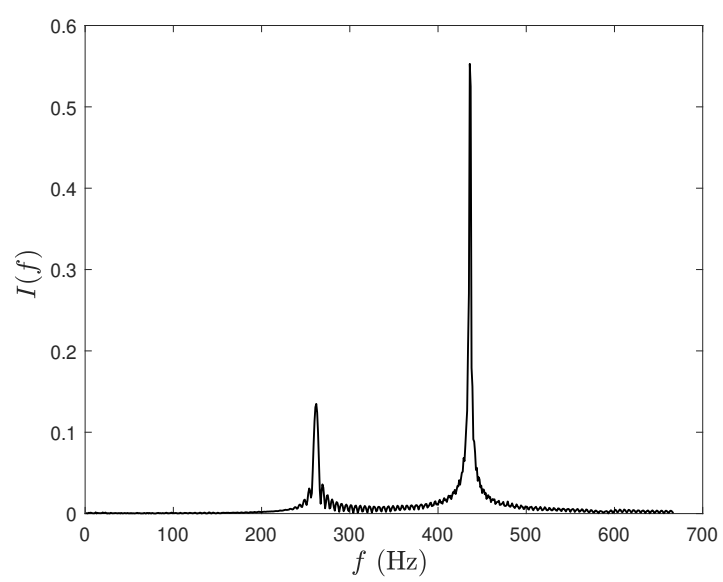

(a)

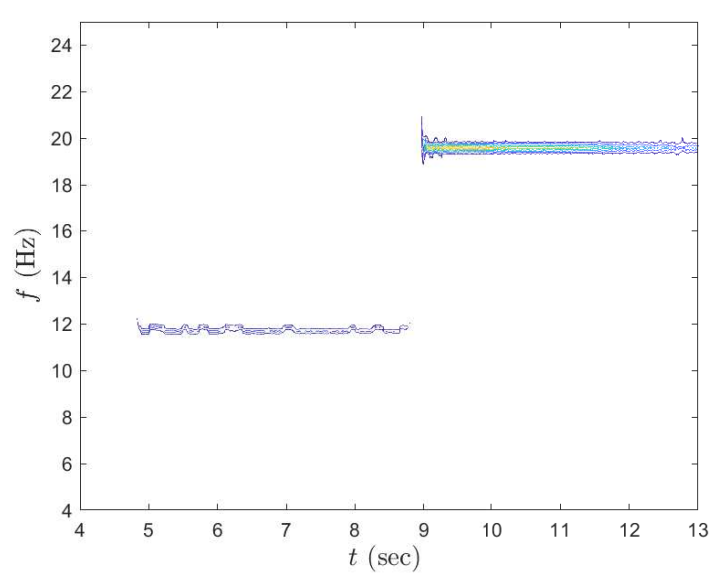

(b)

Figure 3.17: The mass drop experiment: (a) Fourier transform and (b) SST of the signal. 


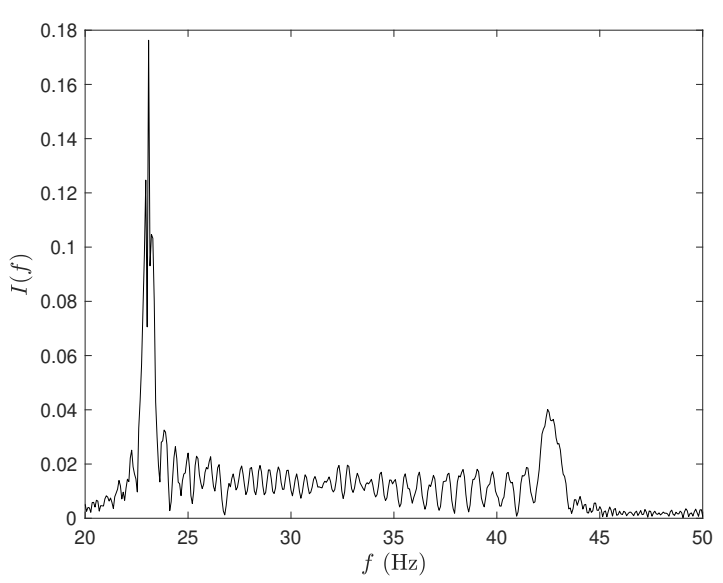

(a)

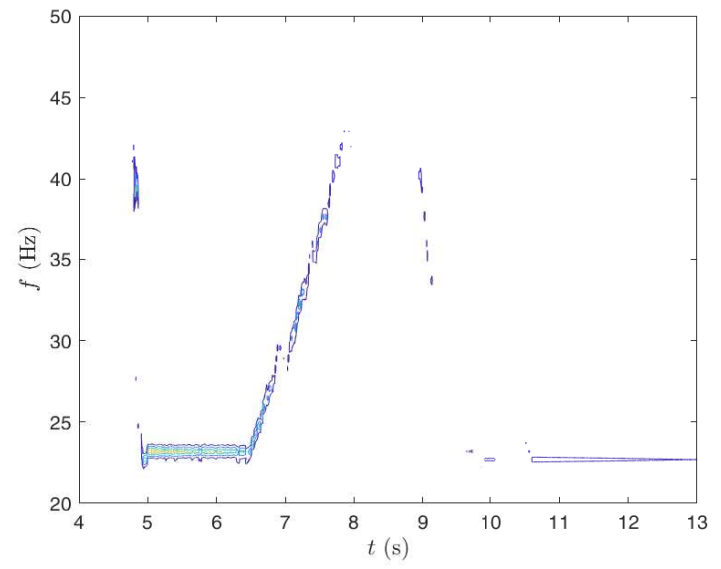

(b)

Figure 3.18: The moving roller experiment: (a) Fourier transform and (b) SST of the signal.

\subsection{Full-scale Validation}

\subsubsection{Details of full-scale data}

The benchmark building at the Massachusetts Institute of Technology campus, known as Green Building have 21 stories above ground with a height of 83.7 meters and a basement of 3.8 meters. The building was instrumented with 36 sensors and the measured acceleration data is used to perform system identification. The sensor locations in the building are shown in Fig. 3.19. The benchmark data [127] contains four ambient data sets, one unidentified event, one firework event, and one earthquake event. For the purpose of this research, one ambient (D1) and the earthquake (D7) data sets are utilized to illustrate the identification of the time-varying frequencies. This time varying characteristics of the data is presented to show change in the frequency. The details of the structure and data acquisition can be found from $[128,127]$ and are not repeated here. 


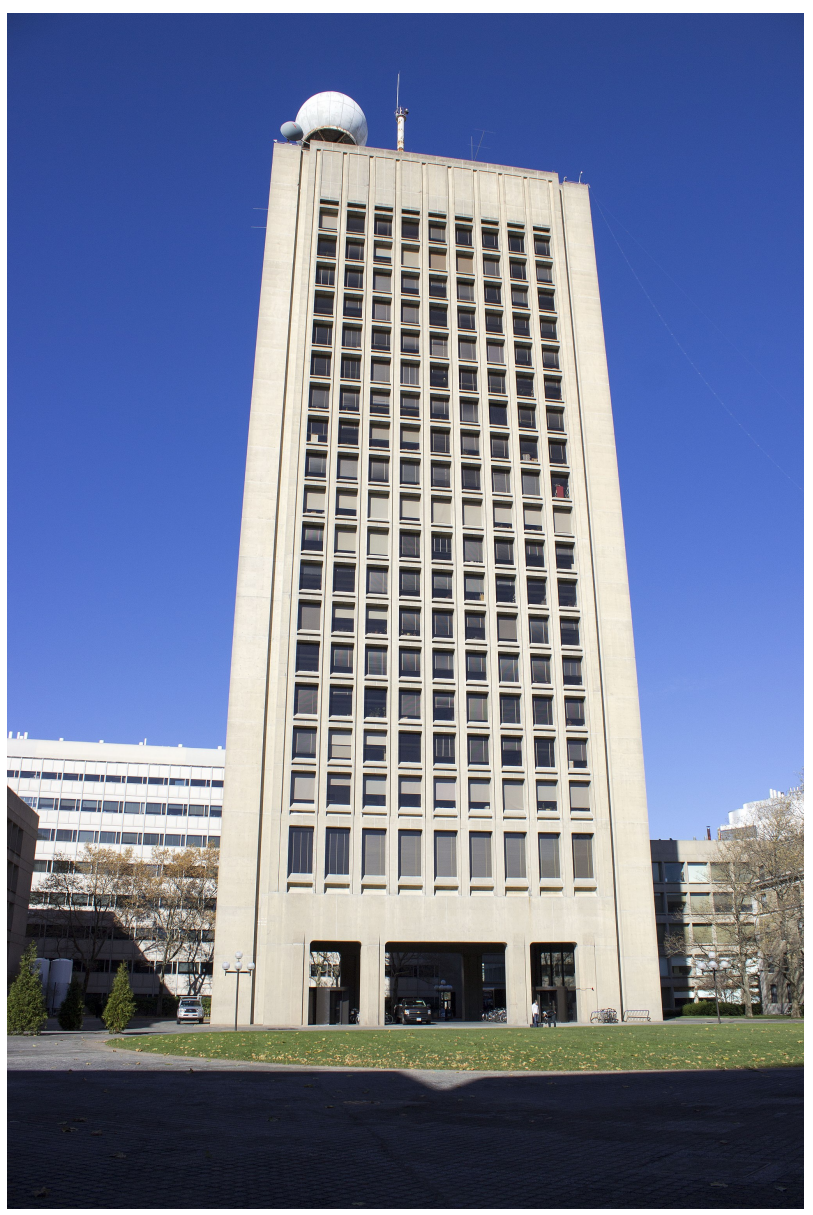

(a)

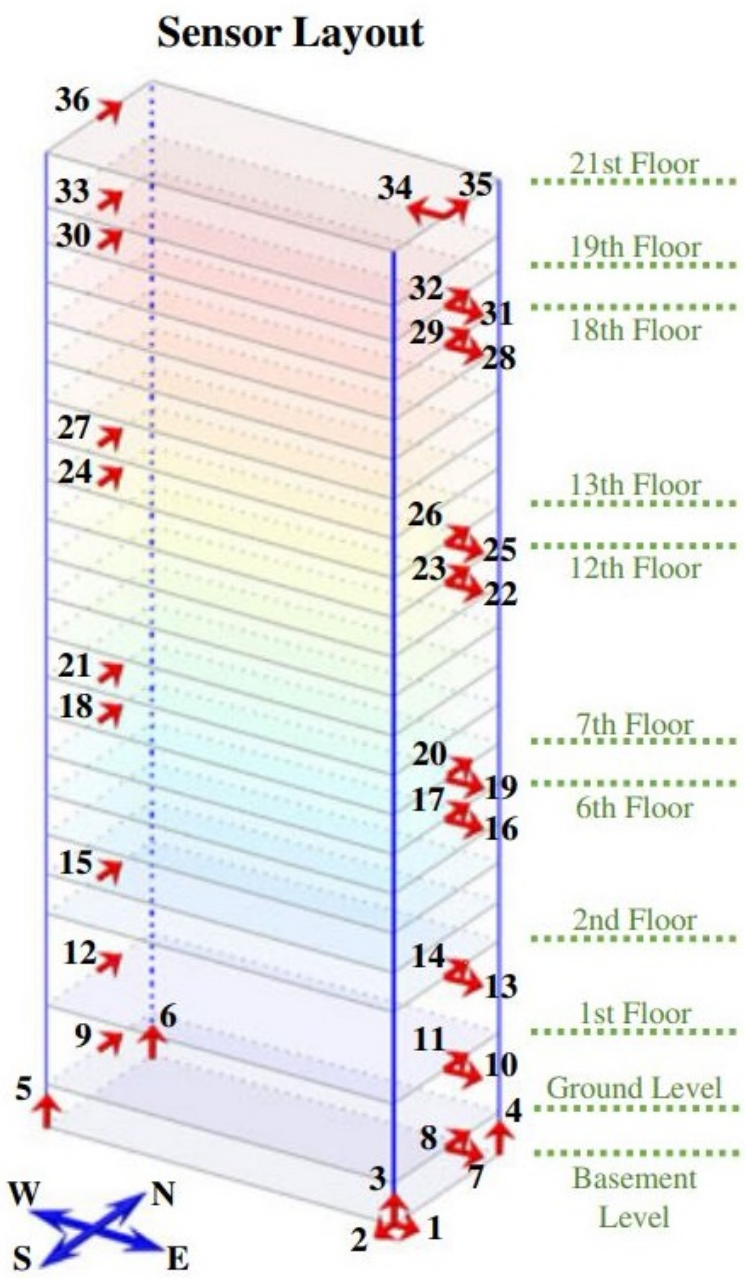

(b)

Figure 3.19: (a) MIT Green building (b) sensor locations in the building (Sun and Buyukozturk 2018).

\subsubsection{Identification results}

The results are shown in Fig. 3.20 and Fig. 3.21 for ambient and earthquake case, respectively. For example, Fig. 3.20(a) shows the Fourier spectra of the identified frequencies and Fig. 3.20(b) shows their SST representation. The frequencies identified from the proposed methods are tabulated with the frequencies available in the literature [127] and are shown in Table 3.2. It is evident that the proposed method is able to correctly evaluate 
the identified frequencies and concur with the results from the existing literature [31]. One of the higher frequency in case of ambient measurement has been reduced from $5.06 \mathrm{~Hz}$ to $3.61 \mathrm{~Hz}$, which can be observed in the SST of the measured data. The SST for higher modes have not been in par with its performance for lower modes and are also seen in SST results of the earthquake-induced response.
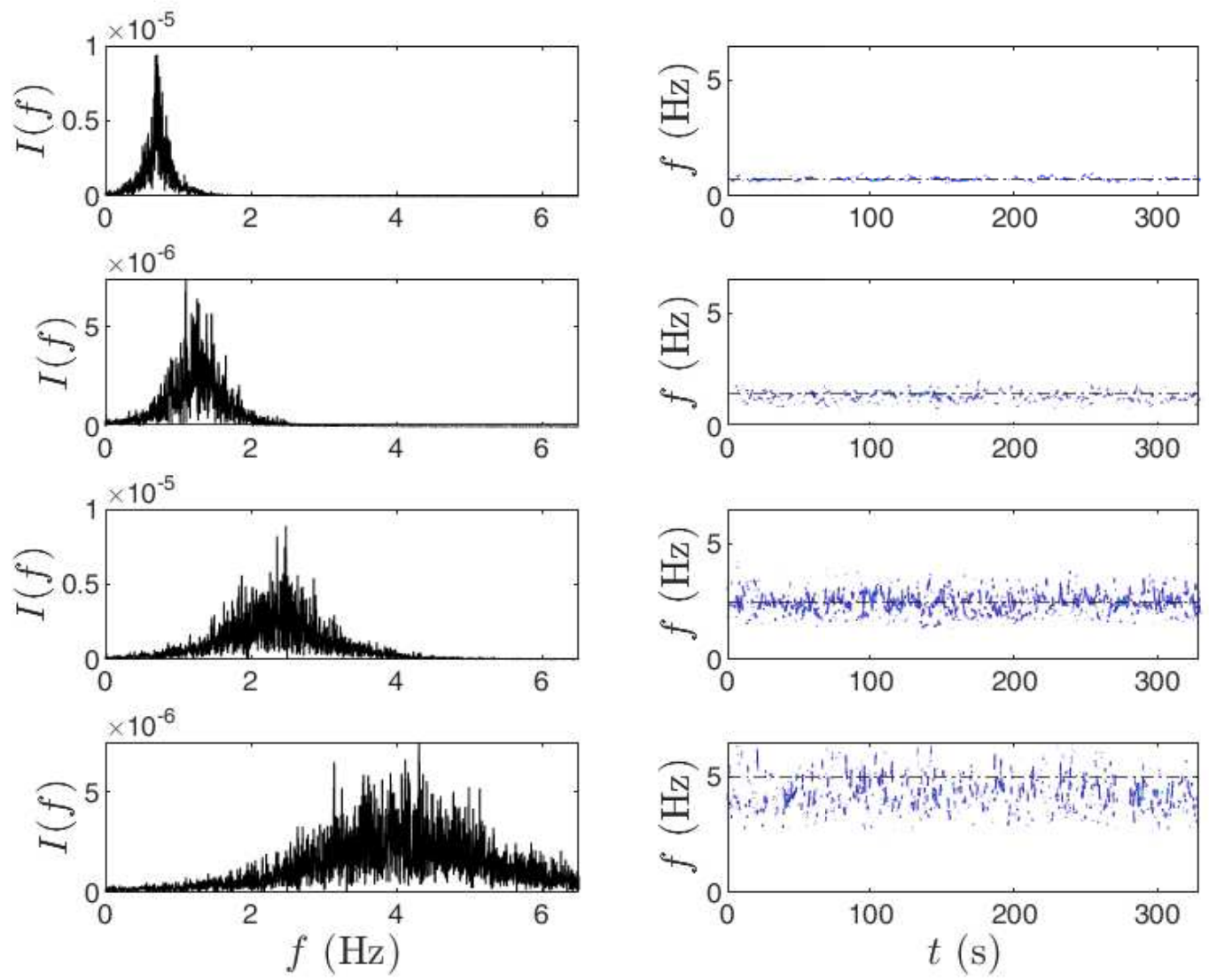

(a)

(b)

Figure 3.20: (a) Fourier spectra of IMFs (b) SST of IMFs obtained for ambient excitation. 

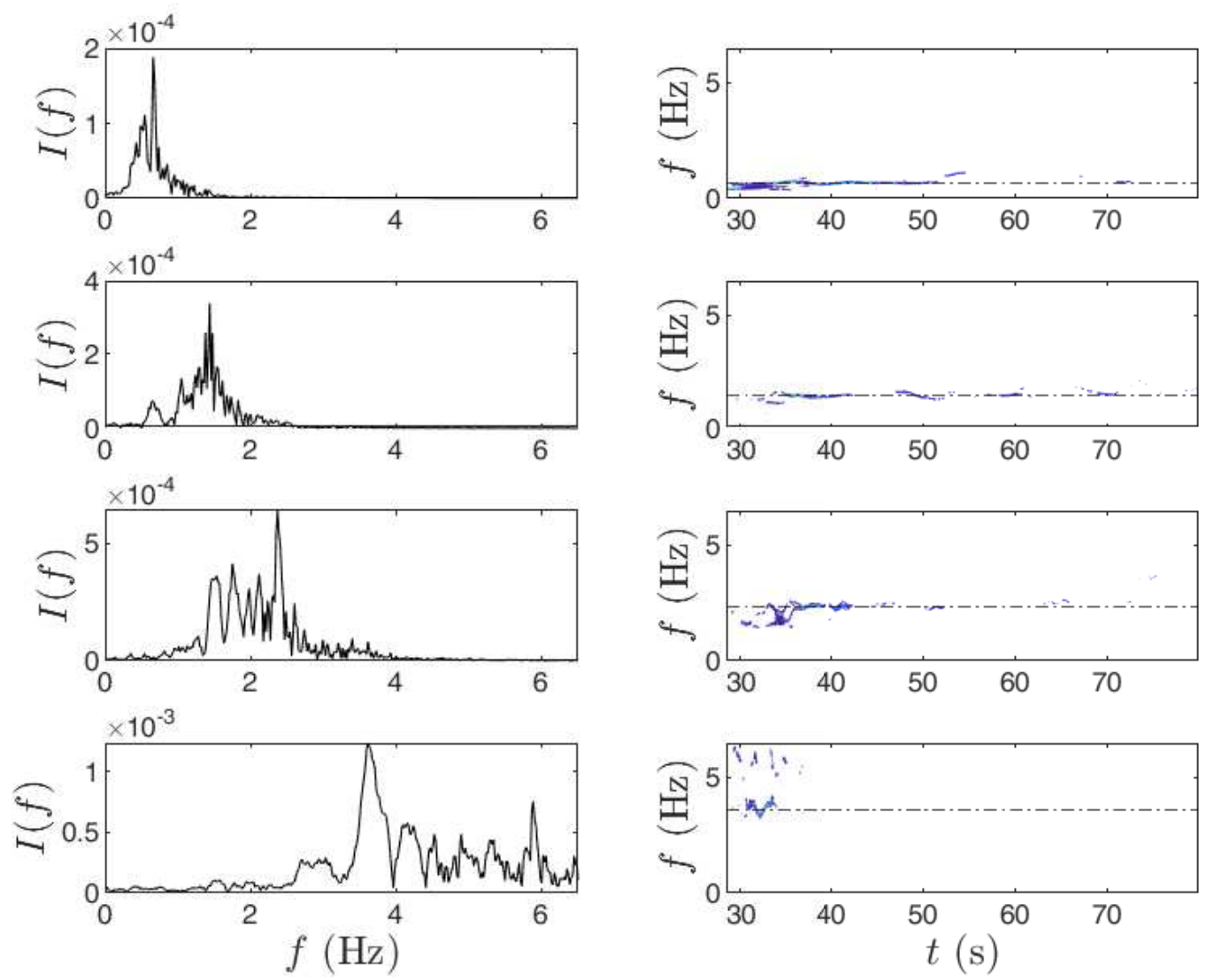

(a)

(b)

Figure 3.21: (a) Fourier spectra of IMFs (b) SST of IMFs obtained for earthquake excitation.

Table 3.2: Identified results $(\mathrm{Hz})$ based on the measurement along East-West direction.

\begin{tabular}{|c|c|c||c|c|}
\hline Mode \# & Ambient & Identified & Earthquake & Identified \\
\hline \hline 1st & 0.70 & 0.70 & 0.66 & 0.66 \\
\hline 2nd & 2.54 & 2.47 & 2.33 & 2.37 \\
\hline 3rd & 5.06 & 4.93 & 3.61 & 3.61 \\
\hline Torsional & 1.46 & 1.46 & 1.44 & 1.44 \\
\hline
\end{tabular}




\subsection{Summary}

A hybrid TF method is proposed by integrating MEMD with SST to track the timevarying modal frequencies of the structure. The efficiency of SST under various frequency resolutions and damage duration are compared with other methods such as CCWT. The proposed method is shown to have the capability of separating modal responses and, subsequently, identifies the time-varying behavior of the structure with both discrete and

progressive damages. A suite of numerical studies is performed to show the robustness of SST for improved TF representation under the different extent of measurement noise and structural damages. Both experimental and full-scale studies show the adequacy of the proposed method for the identification of a time-varying system. While integrating SST with MEMD, it is now possible to perform damage detection using multichannel measurements. 


\section{Chapter 4}

\section{Damage Localization using}

\section{MEMD-based Nonparametric}

\section{Approach}

In this chapter, a novel MEMD method is explored for damage localization using a limited number of sensors. The mode decomposition capability of MEMD and modal energy of damaged and undamaged structural component is compared to identify local changes in the structure. In the context of SHM of large-scale structures, the financial burden of heavy instrumentation restricts its widespread implementation. This study evaluates the possibility of using limited sensors to identify local damages in structures while providing same information as large number of sensors. A suite of numerical studies are used to validate the proposed algorithm. A full-scale real bridge (i.e., Z24 Bridge) is then used to localize the damage. Three different cases of varying severity of damage in a bridge pier is 
used to validate the proposed method.

\subsection{Introduction}

There have been continuous improvements in sensors and sensing technology as they constitute a significant portion of cost during SHM applications and contribute to the overall accuracy of condition assessment. Traditionally, wired sensors are used as a dense array distributed over the structure to acquire long-term SHM data. However, this approach is not a cost-effective and viable option for large-span bridges or tall-buildings due to labourintensive cable installation. The setbacks of wired sensors were envisioned to solve using smart wireless sensors [89],[5]. Capabilities and local processing of wireless sensors in the decentralized framework were much later exploited by [122]. Recently, other modern sensors such as cameras, robotic sensors, smartphones, and drones have been used for SHM through the processing of images and videos [45],[120]. However, such SHM approach is cost-effective only if the hidden structural information and damage characteristics are accurately assessed from the limited measured data.

An optimal number of limited sensors [79] that can provide the same information as the large array of sensors will reduce the cost of SHM. There has been considerable research on system identification by limited sensors using two popular methods, non-sparse and sparse techniques. Non-sparse methods have gained interest in the operational modal analysis as a non-parametric alternative to the structural identification from output-only measurements

[108]. In [60], a modified cross-correlation method was used in combination with EMD.

The authors stated that as low as three sensors provide a good degree of confidence in 
estimating twelve dominant modes; however, efficiently selecting the initial mode-shape matrix remains a challenge. Further, a parallel factor decomposition along with Bayesian model updating was employed for underdetermined modal identification [1]. However, the assumption of stationary response does not work well with the measured responses obtained from civil structures. Additionally, [57] proposed spatial time-frequency distribution to handle nonstationary response. However, the proposed method highly depends on the quality of the selection of a single auto spectral component, and its poor selection could lead to inaccurate results.

Unlike non-sparse methods, sparse techniques utilize TF methods for sparse representation of signals. [140] used sparse component analysis (SCA) along with L1- minimization to improve underdetermined modal identification. The study by [146] explored SCA-based methods in the TF domain to estimate time-varying modal parameters and validated the proposed method under thermal effects. Amini and Hedayati (2016) used STFT and SL0 algorithm to perform underdetermined system identification on earthquake and ambient vibration and showed the robustness under noise to separate closely spaced modes. Moreover, in [142], the authors explored SCA for modal identification using limited sensors and validated on the both stationary and nonstationary response of structures. [28] proposed frequency banding for largely underdetermined scenarios by decomposing a large underdetermined problem into several overdetermined problems. The method operates directly in the frequency domain and analyzes the cross-spectral matrix of the data. However, user intervention during frequency banding and manual selection of estimated modes is one of the limitations of the proposed method. [144] proposed a novel SCA method for esti- 
mating the number of active modes using statistical properties of normalized single source point vector. Although the above sparse and non-sparse system identification methods show a wide variety of applications in modal identification using limited sensors, damage localization has not given its due attention under limited sensor measurements [49].

[10] recently used the system Markov parameters for damage detection. The authors evaluated the effect of noise, number, and location of the sensors. The study was based on finite element study and pre-defined damage locations and did not identify the unknown damage location. [17] used an optimization algorithm to localize and quantify damage in seismically excited structures using a limited number of sensors. A competitive optimization algorithm combined with moment generating function was used as a damage indicator. The limitation of the study lies in using the moment of the segment as a damage indicator while identifying the instance of the damage. Recently, [71] used mode shapes coupled with wavelet transform for damage identification while employing a limited number of sensors. The optimal spatial location of the sensors was achieved using the minimization of the non-diagonal entries in the modal assurance criterion matrix. The study is dependent explicitly on mode shapes, and ambiguity arises on interpreting the damage location with respect to different damage scenarios. Moreover, there is a significant challenge in accurate identification of mode shapes using real data with measurement noise.

In this chapter, a MEMD-based method damage localization is proposed by taking advantage of the modal energies of individual modes extracted from the sensors. Due to the capability of handling a limited number of sensors, MEMD [107],[118] is further explored to compare the performance of the damage localization using a suite of limited 
sensors. The chapter is organized as follows. Section 4.1 provides a brief introduction to this topic, illustrating the existing literature and the gap areas. Section 4.2 presents the proposed methodology and the corresponding theoretical background. Section 4.3 shows the numerical studies using a 10-DOF model subjected to a wide extent of damages and measurements obtained from a suite of limited sensors. A full-scale study comprising of a real bridge with two different damage scenarios is presented in Section 4.4. Finally, Section 4.5 presents the conclusions and future scope of research on this topic.

\subsection{Proposed Algorithm}

Consider a linear dynamical system with $n$ degree-of-freedom (DOF) subjected to a broadband excitation $\mathbf{X}(\mathbf{t})$, the equation of motion is expressed as,

$$
\mathbf{M} \ddot{\mathbf{y}}(t)+\mathbf{C} \dot{\mathbf{y}}(t)+\mathbf{K y}(t)=\mathbf{X}(t)
$$

where $\mathbf{y}(\mathbf{t})$ is the displacement vector. Using the classical modal superposition theorem, The solution to Eq. 4.1 for those of broad-band $\mathbf{X}(t)$ can be written in terms of an expansion of vibration modes:

$$
\mathrm{y}=\boldsymbol{\Phi} \nu
$$

where $\mathbf{y}$ and $\boldsymbol{\nu}$ is the response and modal coordinate matrix, respectively. $\boldsymbol{\Phi}_{m \times n}$ is the modal transformation matrix. $n$ and $m$ is the number of modal responses and measurements, respectively. The measurement at the $i$-th DOF $(i=1,2, \ldots, m)$ can be expressed 
as,

$$
y_{i}(t)=\sum_{j=1}^{n} \phi_{i j} \nu_{j}(t)
$$

where $\nu_{j}$ is the $j$-th modal response and $\phi_{i j}$ represents the mode shape ordinate of $i$-th DOF and $j$-th mode. Since MEMD results in IMFs containing mono-component modal responses, each signal of $\mathbf{y}(\mathbf{t})$ can be expressed in terms of its IMFs:

$$
y_{i}(t)=\sum_{j=1}^{n} I_{i j}(t)
$$

where $I_{i j}$ is the $j$-th IMF of $y_{i}(t)$. Comparing the equality of Eq. 4.3 and Eq. 4.4, we get:

$$
I_{i j}(t)=\phi_{i j} \nu_{j}(t) \quad i=1,2, \ldots, m
$$

Each IMF, $I_{i j}$ is then analyzed to obtain Fourier spectrum $\left(E_{i j}(f)\right)$, as shown in Eq. 4.6,

$$
E_{i j}(f)=\int_{-\infty}^{\infty} I_{i j}(t) e^{-k f t} d t
$$

If $E_{i j}(f)$ is the Fourier transform of $I_{i j}(t)$, then $\left|E_{i j}(\tau)\right|^{2}$ can be interpreted as energy density at the frequency $\tau$, which means that the total energy contained in a small frequency interval $[\tau-\epsilon, \tau+\epsilon]$ around $\tau$ is approximately given by $2 \epsilon\left|E_{i j}(\tau)\right|^{2}$. However, as per mathematical axiom, if $|x|>|y|$, then, for every, $x \neq 0, y \neq 0$, and $x, y>0, x^{2}>y^{2}$ is true, and thus, the peak Fourier amplitude can be interpreted as modal energy. The damage localization feature is then extracted using percentage change in peak amplitude of Fourier spectrum of modal responses as shown in Eq. 4.7, 


$$
\Delta E_{i j}=\left|\frac{\left(E_{i j}\left(f_{D}\right)-E_{i j}\left(f_{U}\right)\right)}{E_{i j}\left(f_{U}\right)}\right| * 100
$$

where $\Delta E_{i j}$ represents absolute percentage change in modal energy of the corresponding damaged, $f_{D}$ and undamaged, $f_{U}$ modal frequencies. Then, $\Delta E_{a i}$ is calculated as the average of $\Delta E_{i j}$ for all selected IMFs of $i$-th sensor,

$$
\Delta E_{a i}=\frac{\sum_{j=1}^{n} E_{i j}}{n}
$$

Finally the threshold $\overline{\Delta E}$ is proposed by taking mean value of $\Delta E_{a i}$ for all the selected sensor locations,

$$
\overline{\Delta E}=\frac{\sum_{i=1}^{m} E_{a i}}{m}
$$

The proposed algorithm is applied by first decomposing the multi-sensor data into their IMFs, and their Fourier spectra are used to identify $\Delta E_{i j}$ of the corresponding frequencies and are used as damage localization feature. The damage is deemed present for any sensor location, if $\Delta E_{a i}$ is higher than $\overline{\Delta E}$. The proposed algorithm is presented in Algorithm 3 .

\subsection{Numerical Studies}

A 10-DOF model is used to illustrate the performance of the proposed method. The model is subjected to two separate broadband earthquakes (EQ), Imperial Valley EQ (with a PGA of 0.1g) and Borrego Mountain EQ (with a PGA of 0.05g), to study the damage 


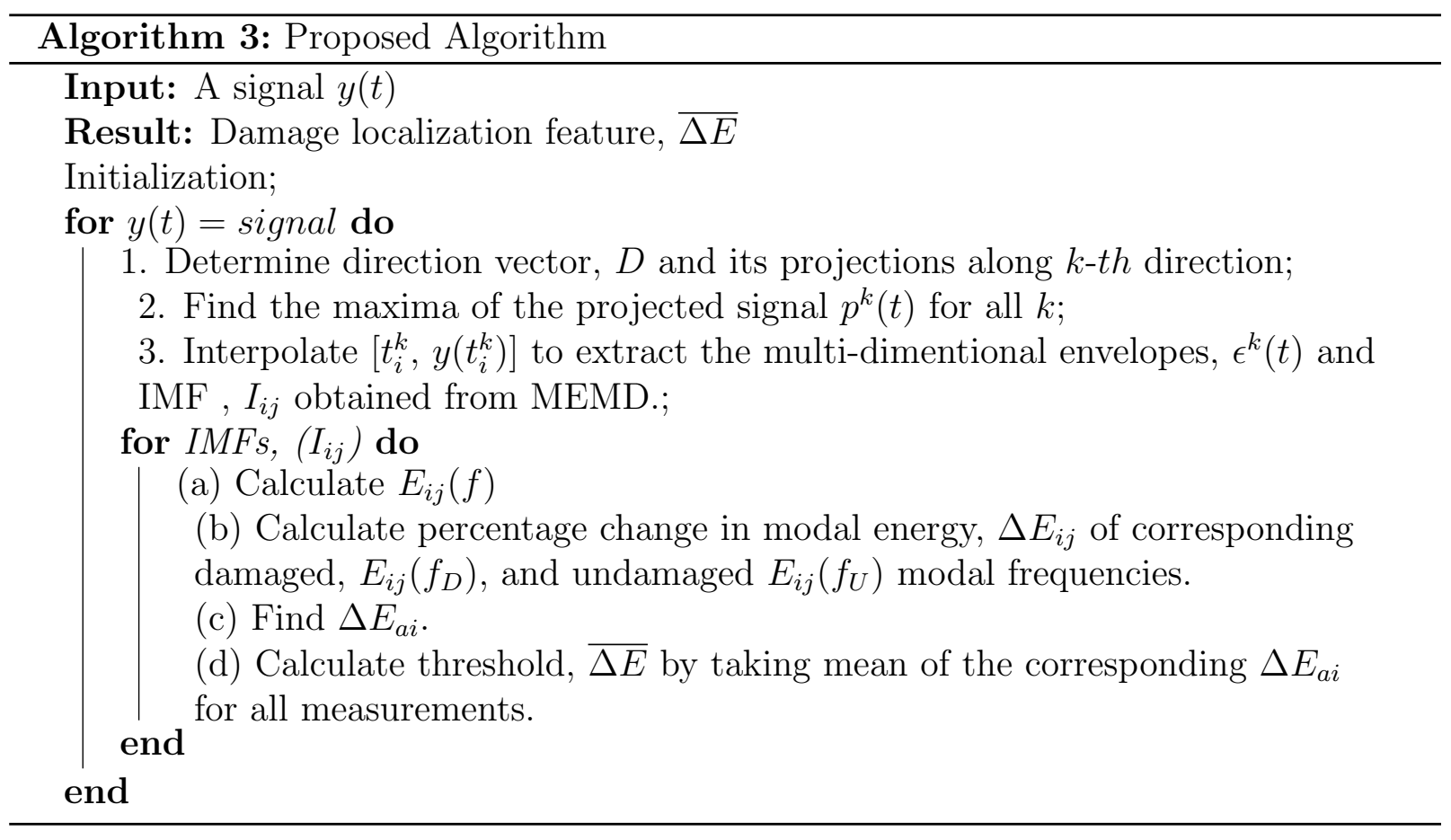

localization using the earthquake-induced measurements. Considering the fact that, the first floor columns are primarily damaged during the earthquakes, three damage scenarios are used to evaluate the performance of the proposed method under varying level of damage in the first floor. The different damage cases are shown in Table 4.1. For example, C1 indicates undamaged model with first five natural frequencies as $0.78,1.8,2.83,3.88$ and $4.96 \mathrm{~Hz}$, respectively, whereas $\mathrm{C} 2$ represents the 10-DOF model with $20 \%$ damage in the column of the first floor. Table 4.2 illustrates the corresponding natural frequencies of the 10-DOF model under $\mathrm{C} 2$ and $\mathrm{C} 3$, and the associated percentage changes in the frequencies with respect to $\mathrm{C} 1$. It can be observed that the damage cases are chosen such a way that the overall average reduction in modal frequencies are less than $5 \%$ in $\mathrm{C} 2$ and $\mathrm{C} 3$ (i.e., $1.9 \%$ and $4.5 \%$, respectively), where the traditional TF methods (Sony and Sadhu 2020) were unable to delineate such minor change in the modal frequencies. The models are subjected to the above earthquakes separately and the earthquake-induced responses are 
analyzed to identify the location of the simulated damage.

Table 4.1: Varying level of damage in the first floor and the associated acronyms.

\begin{tabular}{|c|c|c|c|}
\hline$i$ & $\mathrm{C} 1$ & $\mathrm{C} 2$ & $\mathrm{C} 3$ \\
\hline \% damage & 0 & 20 & 40 \\
\hline
\end{tabular}

\subsubsection{Vibration data induced by Imperial Valley earthquake}

The 10-DOF model is excited using Imperial Valley earthquake and damage is induced at the first floor $(i=1)$ by reducing the stiffness, and proposed algorithm is evaluated to localize damage using a combination of all the sensors. The Fourier spectra of the typical floor responses for different damage cases are shown in Fig. 4.1. MEMD is implemented to decompose the simulated responses into their IMFs. The first two modes are illustrated to show the change in the frequencies and $\Delta E_{a i}$ is used as a damage indicator. The values of $\Delta E_{i j}$ for various floors of $I_{i 1}$ (IMF-1) and $I_{i 2}$ (IMF-2) are tabulated in Table 4.3 and Table 4.4, respectively. For example, Table 4.3 provides the peak Fourier amplitude of first floor response as 8.2 unit for C1, as shown in Fig. 4.2(a). In Figs. 4.2, 4.3, and 4.4, the first

Table 4.2: The first five modal frequencies of the 10-DOF model for various damage cases and their percentage reduction with respect to $\mathrm{C} 1$.

\begin{tabular}{|c|c|c|c|c|c|}
\cline { 2 - 6 } \multicolumn{1}{c|}{} & \multicolumn{5}{|c|}{ Modal frequencies $(\mathrm{Hz})$} \\
\cline { 2 - 6 } & $f_{1}$ (\% change) & $f_{2}$ (\% change) & $f_{3}$ (\% change) & $f_{4}(\%$ change) & $f_{5}$ (\% change) \\
\hline C2 & $0.76(2.6)$ & $1.76(2.2)$ & $2.78(1.8)$ & $3.82(1.5)$ & $4.90(1.2)$ \\
\hline C3 & $0.73(6.4)$ & $1.70(5.9)$ & $2.71(4.2)$ & $3.75(3.4)$ & $4.83(2.6)$ \\
\hline
\end{tabular}




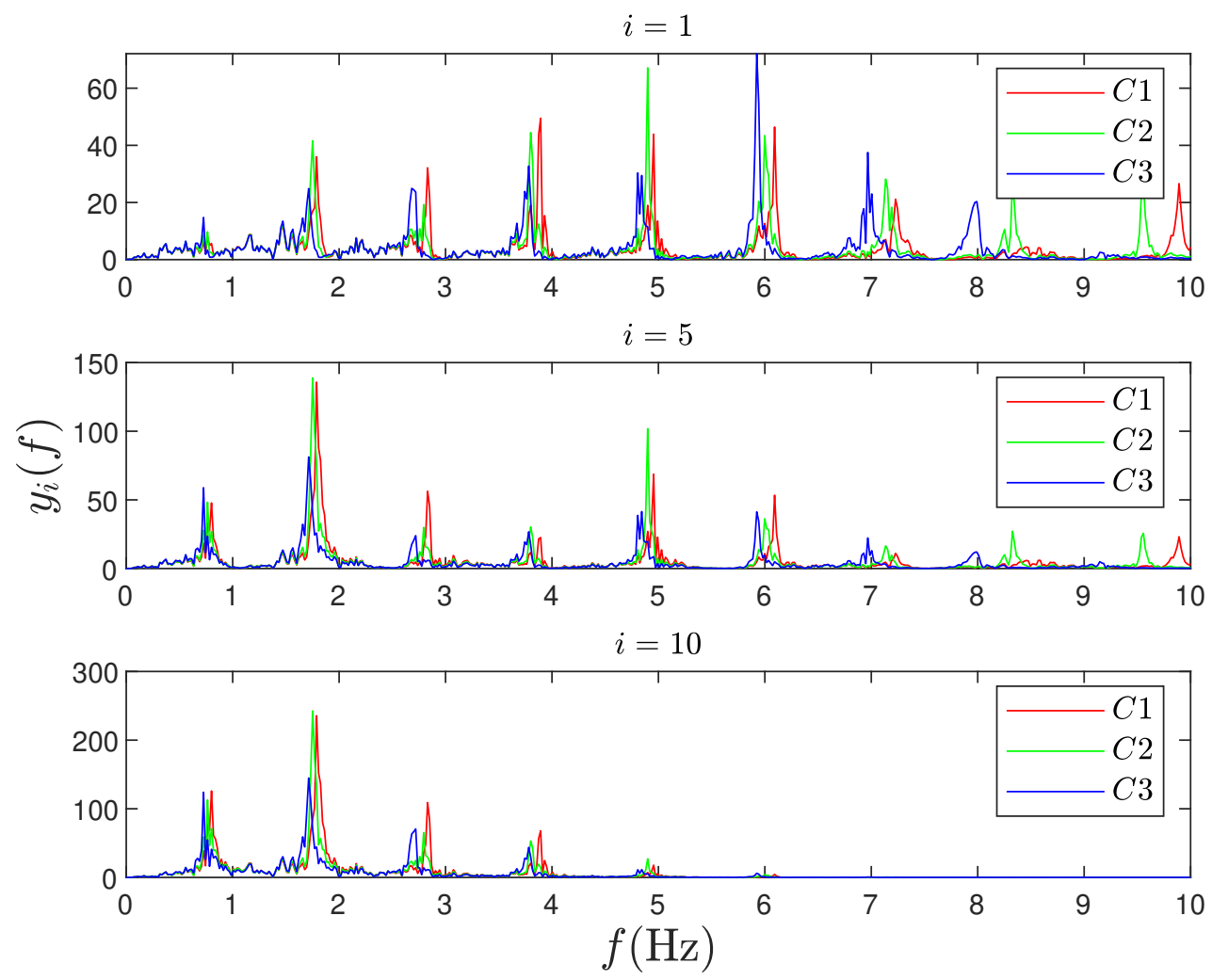

Figure 4.1: Fourier spectra of raw data of the 10-DOF model under various damage cases.

row shows IMF-1 and second row shows IMF-2. The corresponding modal energies for C1, C2, and C3 are shown in Figs. 4.2, 4.3, and 4.4, respectively.

To localize the damage, Eq. 4.7 is used to calculate $\Delta E_{i j}$, followed by evaluating $\Delta E_{a i}$ using Eq. 4.8 for all selected IMFs of a sensor. Finally, the average of $\Delta E_{a i}$ for all sensors is evaluated using Eq. 4.9 and is used as a damage localization feature, as presented in Table 4.4. The percentage change between $\mathrm{C} 1$ and $\mathrm{C} 2$ is $6.1 \%$ for IMF-1, similarly, for $\mathrm{C} 1$ and C3, its $51.2 \%$. The damage localization feature is then evaluated by taking mean of $\Delta E_{i j}$ of all IMFs. For example, $\mathrm{C} 2$ yields $\Delta E_{a i}$ as 32.5 unit, which is equal to average of corresponding values of IMF-1 and IMF-2. $\Delta E_{a i}$ values for various floors are shown in Fig. 4.5(a). For example, the first floor has a value of 32.5 unit, the fifth floor has a value of 
22.7 unit, and tenth floor has a value of 20.7 units. In this sub-figure, $\overline{\Delta E}$ is calculated as 25.3 unit, which is shown in the dotted line and it can be clearly seen that the proposed damage index exceeds $\overline{\Delta E}$ at $i=1$, indicating accurate damage localization at the first floor.

Table 4.3: Modal energies $\left(E_{i j}(f)\right)$ of IMF-1 and IMF-2 of the 10-DOF model subjected to Imperial Valley earthquake.

\begin{tabular}{|c|c|c|c|c|c|c|}
\cline { 2 - 7 } \multicolumn{1}{c|}{} & \multicolumn{2}{c|}{ C1 (Fig. 4.2) } & \multicolumn{2}{c|}{ C2 (Fig. 4.3) } & \multicolumn{2}{c|}{ C3 (Fig. 4.4) } \\
\hline Floor $(i)$ & IMF-1 & IMF-2 & IMF-1 & IMF-2 & IMF-1 & IMF-2 \\
\hline 1 & 8.2 & 20.4 & 8.7 & 32.4 & 12.4 & 26 \\
\hline 5 & 43.6 & 85.1 & 47.1 & 116.9 & 41.9 & 74.4 \\
\hline 10 & 112 & 144.1 & 112.8 & 202.8 & 86 & 130.2 \\
\hline
\end{tabular}

Table 4.4: $\Delta E_{a i}$ for the various floors.

\begin{tabular}{|c|c|c|c|c|c|c|}
\cline { 2 - 7 } \multicolumn{1}{c|}{} & \multicolumn{2}{c|}{ C2-C1 } & \multicolumn{2}{c|}{ C3-C1 } & \multicolumn{2}{c|}{$\Delta E_{a i}$} \\
\hline Floor $(i)$ & $\Delta E_{i 1}$ & $\Delta E_{i 2}$ & $\Delta E_{i 1}$ & $\Delta E_{i 2}$ & C2 (Fig. 4.5 (a)) & C3 (Fig. 4.6 (a)) \\
\hline 1 & 6.1 & 58.8 & 51.2 & 27.5 & 32.5 & 39.3 \\
\hline 5 & 8.0 & 37.4 & 3.9 & 12.6 & 22.7 & 8.2 \\
\hline 10 & 0.7 & 40.7 & 23.2 & 9.6 & 20.7 & 16.4 \\
\hline
\end{tabular}



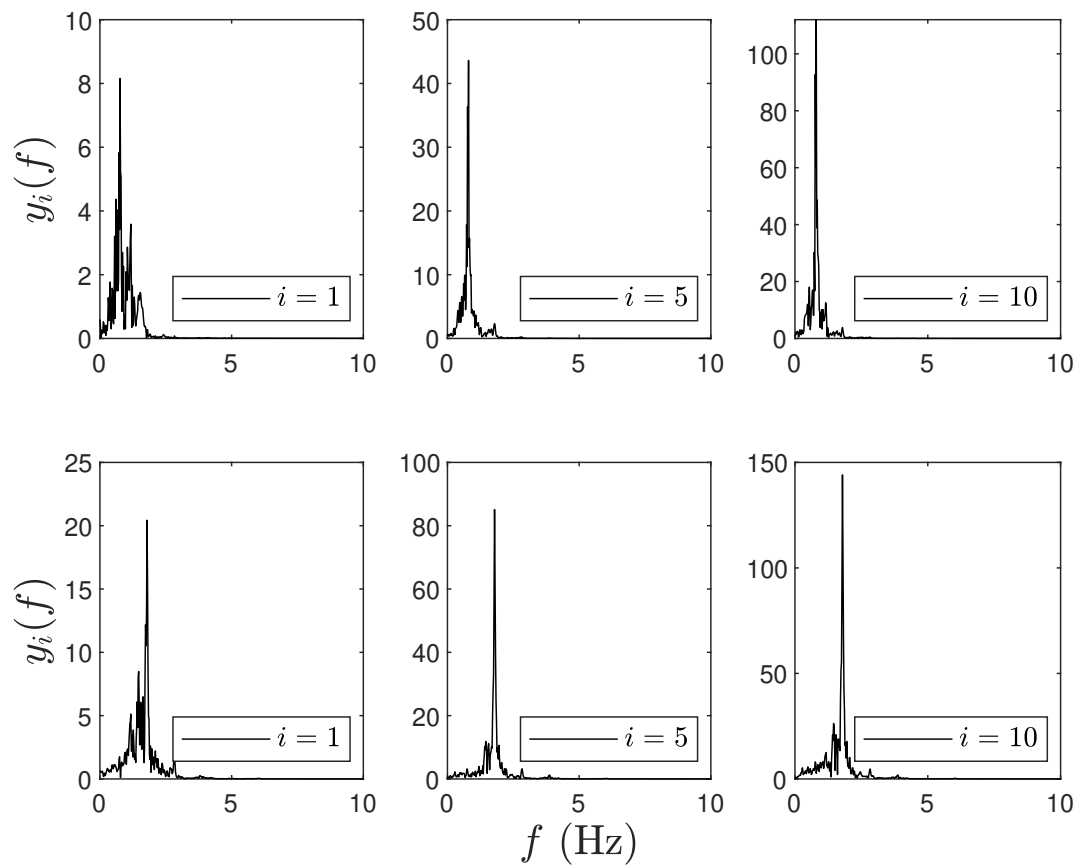

Figure 4.2: Fourier spectra of IMF-1 and IMF-2 of the 10-DOF model for C1.
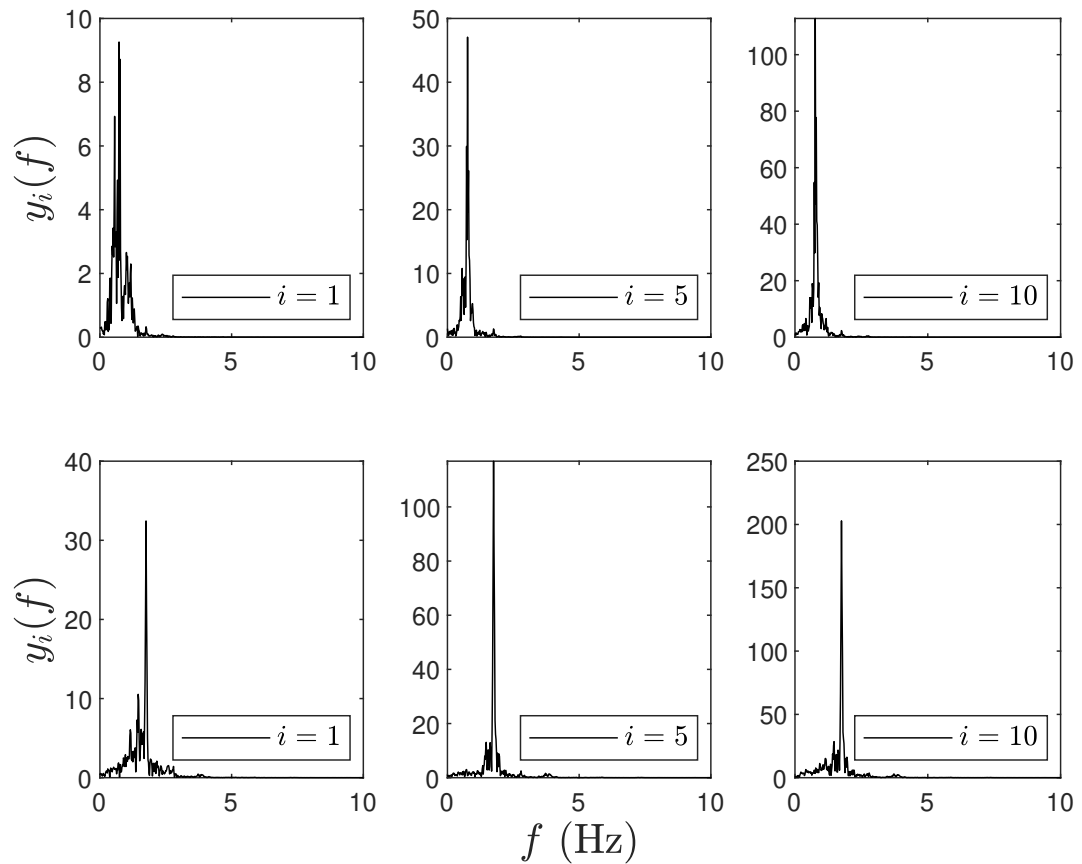

Figure 4.3: Fourier spectra of IMF-1 and IMF-2 of the 10-DOF model for C2. 

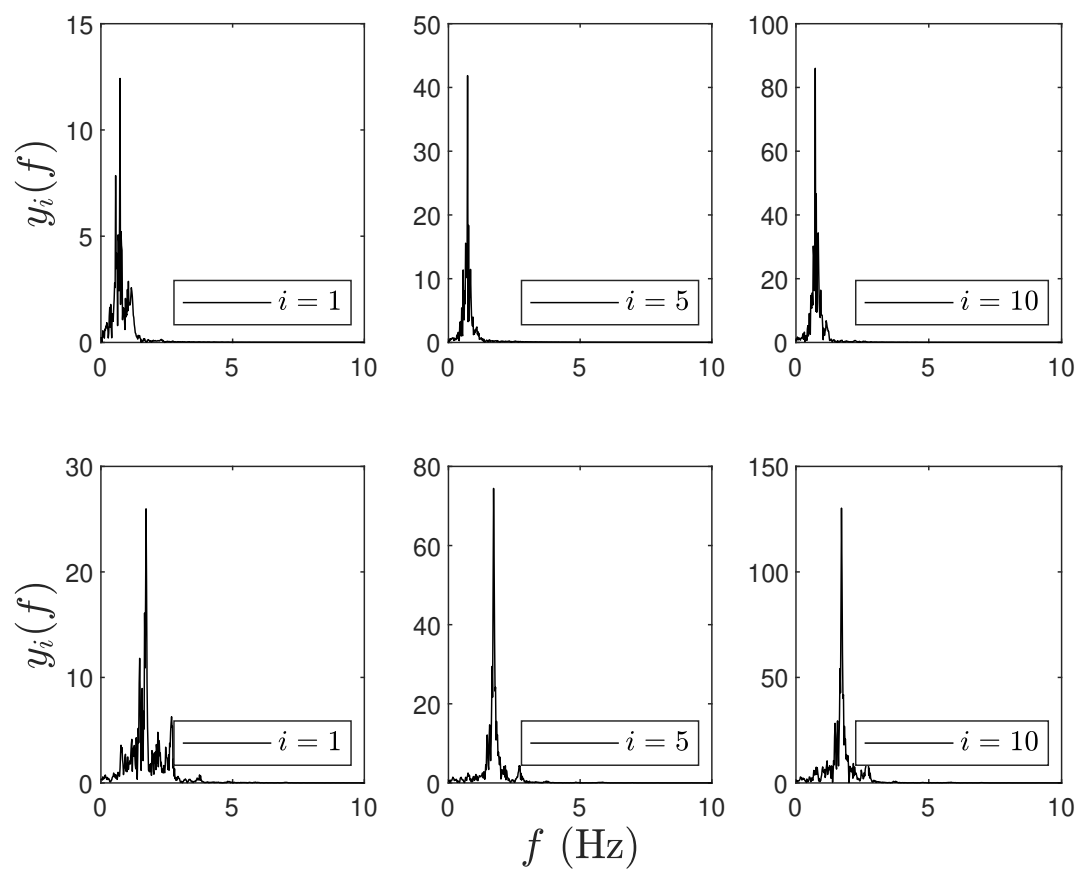

Figure 4.4: Fourier spectra of IMF-1 and IMF-2 of the 10-DOF model for C3.

The study is further enunciated by considering a limited number of sensors while keeping sensor locations 1, 5 and 10 intact for all the cases to have consistent reference sensors for the comparison purposes. For example, Fig. 4.5 shows the identification results of $\mathrm{C} 2$ for a limited sensor cases using 10, 9, 7, and 5 sensors, respectively. The damage location can be clearly identified in floor 1, however, in case of 8 and 6 sensors, the proposed method could not classify accurately. In case of C3, all the sensors classify the damaged floor accurately, as shown in Fig. 4.6. Therefore, the sensitivity of the proposed method improves with the severity of the damage. 
(a)

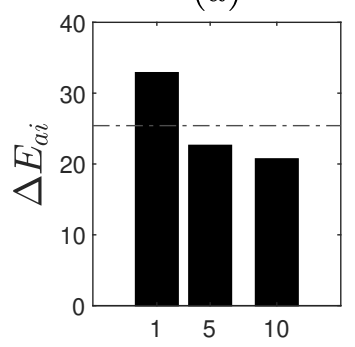

(d)

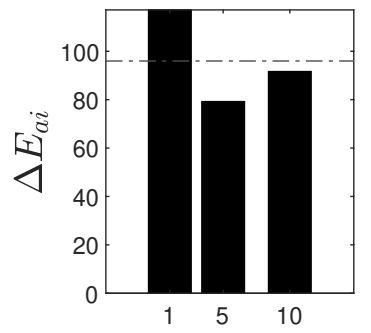

(b)

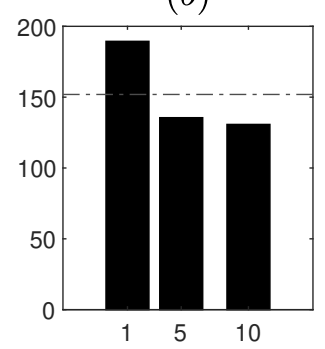

(e)

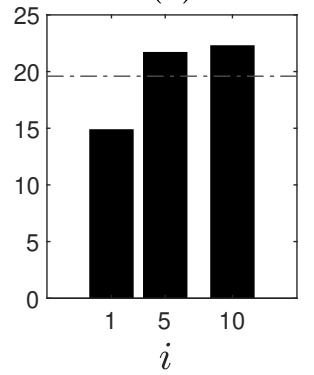

(c)

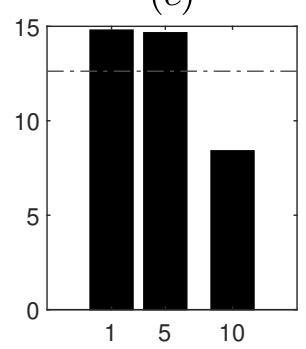

(f)

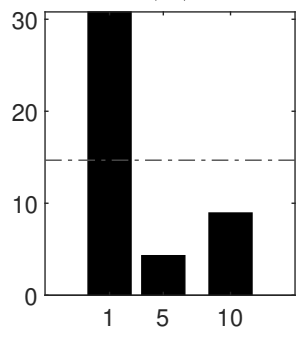

Figure 4.5: $\Delta E_{a i}$ of IMFs for C2 subjected to Imperial Valley EQ using (a) 10 sensors, (b) 9 sensors, (c) 8 sensors, (d) 7 sensors, (e) 6 sensors, and (f) 5 sensors.

(a)

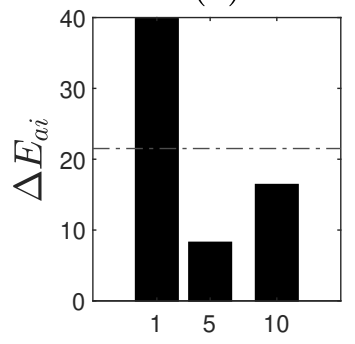

(d)

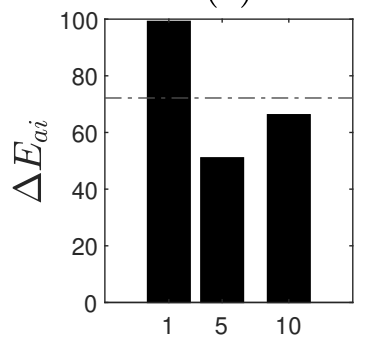

(b)

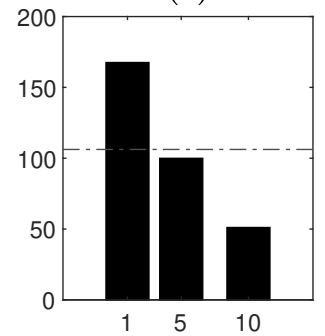

(e)

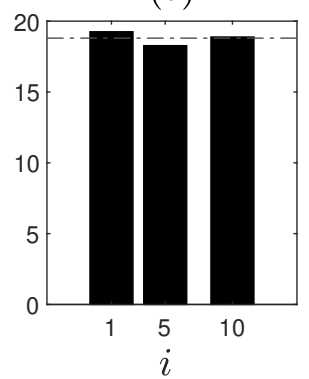

(c)

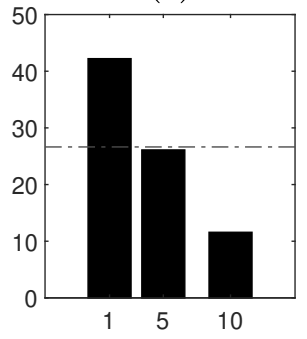

$(f)$

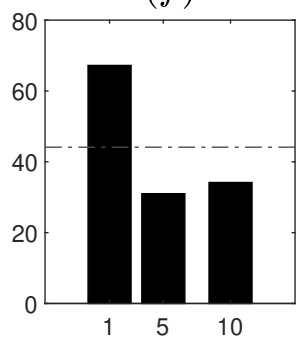

Figure 4.6: $\Delta E_{a i}$ of IMFs for C3 subjected to Imperial Valley EQ using (a) 10 sensors, (b) 9 sensors, (c) 8 sensors, (d) 7 sensors, (e) 6 sensors, and (f) 5 sensors. 


\subsubsection{Vibration data induced by Borrego Mountain earthquake}

The earthquake-induced vibration of the 10-DOF model subjected to another broadband earthquake, Borrego Mountain EQ, is considered to illustrate the performance of the proposed method and study the effect of excitation on the performance of damage localization. For example, Fig. 4.7 shows a clear presence of damage in the first floor in case of $\mathrm{C} 2$ using 5-7, and 9-10 sensors. In case of C3, all the sensors classify the damaged floor accurately, as shown in Fig. 4.8.

Based on the results of section 4.1 and 4.2 , it can be observed that the performance of the proposed method is invariant of the type of excitation. The classification or damage localization accuracy is shown in Table 4.5, where 0 and 1 indicate index for misclassification and accurate classification, respectively. It can be concluded that the proposed method is sensitive to the severity of damage, the higher the severity, the higher is the accuracy for damage localization. However, the proposed method has nearly $92 \%$ accuracy across all limited sensor cases (i.e., 24) in C2 and C3 subjected to two example earthquakes, indicating its efficacy to accurately identify less than $5 \%$ global change in the modal frequencies.

Table 4.5: Damage localization accuracy of the 10-DOF model using the limited number of sensors.

\begin{tabular}{|c|c|c|c|c|c|c|}
\hline No. of sensors & 10 & 9 & 8 & 7 & 6 & 5 \\
\hline \hline C2 - Imperial Valley EQ & 1 & 1 & 1 & 1 & 0 & 1 \\
\hline C3 - Imperial Valley EQ & 1 & 1 & 1 & 1 & 1 & 1 \\
\hline C2 - Borrego Mt. EQ & 1 & 1 & 0 & 1 & 1 & 1 \\
\hline C3 - Borrego Mt. EQ & 1 & 1 & 1 & 1 & 1 & 1 \\
\hline
\end{tabular}


(a)

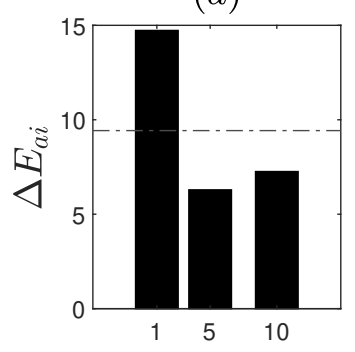

$(d)$

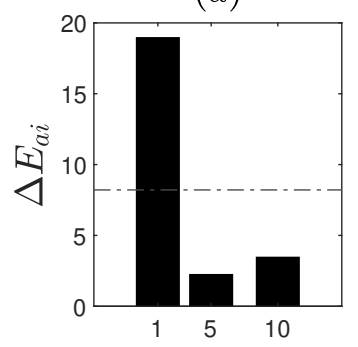

(b)

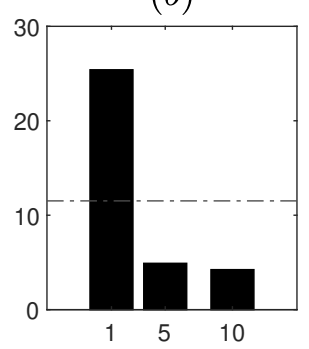

$(e)$

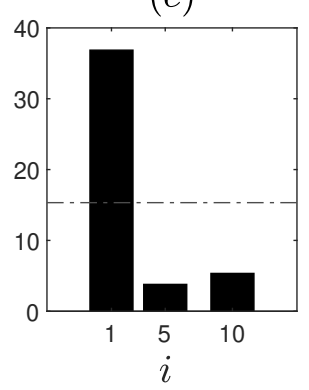

$(c)$
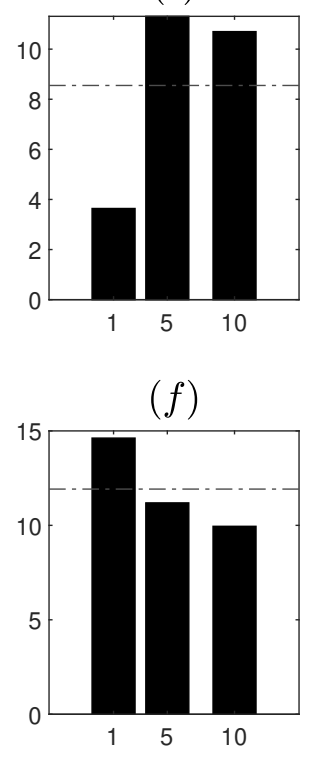

Figure 4.7: $\Delta E_{a i}$ for $\mathrm{C} 2$ subjected to Borrego Mountain EQ using (a) 10 sensors, (b) 9 sensors, (c) 8 sensors, (d) 7 sensors, (e) 6 sensors, (f) 5 sensors.

$(a)$

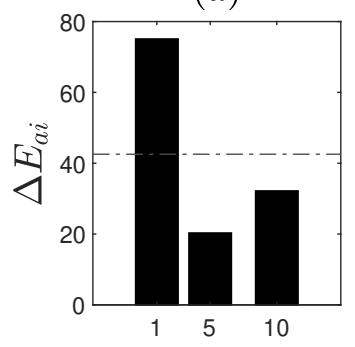

$(d)$

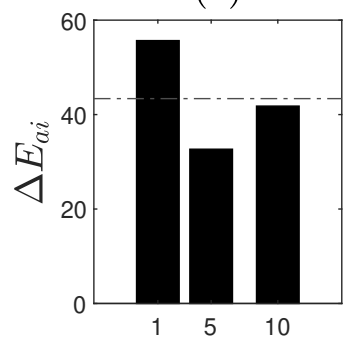

(b)

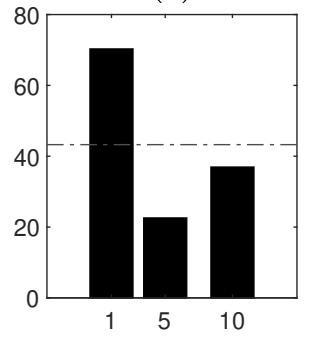

$(e)$

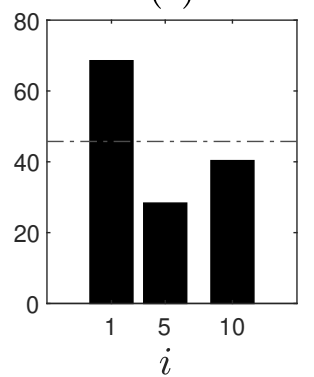

$(c)$
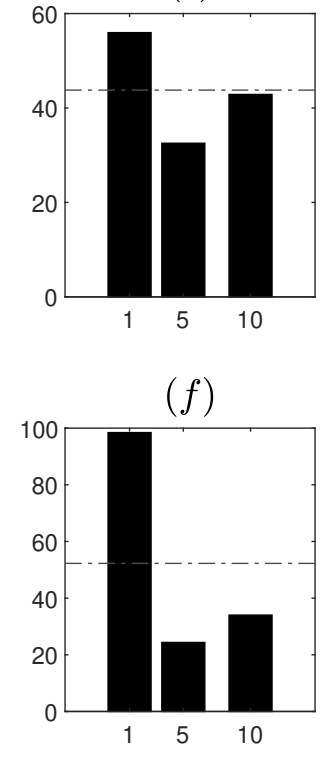

Figure 4.8: $\Delta E_{a i}$ for C3 subjected to Borrego Mountain EQ using (a) 10 sensors, (b) 9 sensors, (c) 8 sensors, (d) 7 sensors, (e) 6 sensors, (f) 5 sensors. 


\subsection{Full-scale Study}

\subsubsection{Details of the full-scale study}

A benchmark study on Z24 bridge is used to evaluate the performance of the proposed method. The bridge is located in Canton Bern, Switzerland, connecting Koppigen and Utzenstorf. The bridge is a highway overpass linking Bern and Zurich, as shown in Fig. 4.9. Z24 bridge is a prestressed bridge, with three spans, two lanes and $60 \mathrm{~m}$ overall length [91, 106]. The study has multiple damage cases, however, for the purpose of the damage localization, only three cases are considered with a total number of eight sensors, as shown in Fig. 4.10. The pier settlement case is used to localize the damage and differentiate the damaged pier from the undamaged pier. The settlement was simulated by cutting the Koppigen pier and removing about $0.4 \mathrm{~m}$ of concrete. Lowering and lifting was applied by six hydraulic jacks. During the tests, the pier rested on steel sections with similar stiffness as the uncut concrete section. The lowering of the pier is carried out by supporting the structure with scaffolding. The pier is cut to support its dead weight, test equipment, and the impact of a vehicle with and without normal force. The base plates are located and connected using shear connectors. Hydraulic jacks are used to support and replacement section is provided and in the end, bond between the rail and concrete is established using prestressing as shown in Fig. 4.11, along with the actual damage induced in the pier. One of the piers (Koppigen) among two piers, as shown in Fig. 4.10, is damaged by lowering the piers. In this study, first an undamaged case is used as a baseline and then, a pier settlement of $20 \mathrm{~mm}, 40 \mathrm{~mm}$ and $95 \mathrm{~mm}$ are used as the damaged cases. Schematic and 
sensors of the undamaged and damaged piers used in this study are shown in Fig. 4.10.

The data was sampled at $100 \mathrm{~Hz}$, and the data was made publicly available by researchers at the Katholieke Universiteit Leuven (https://bwk.kuleuven.be/bwm/z24).

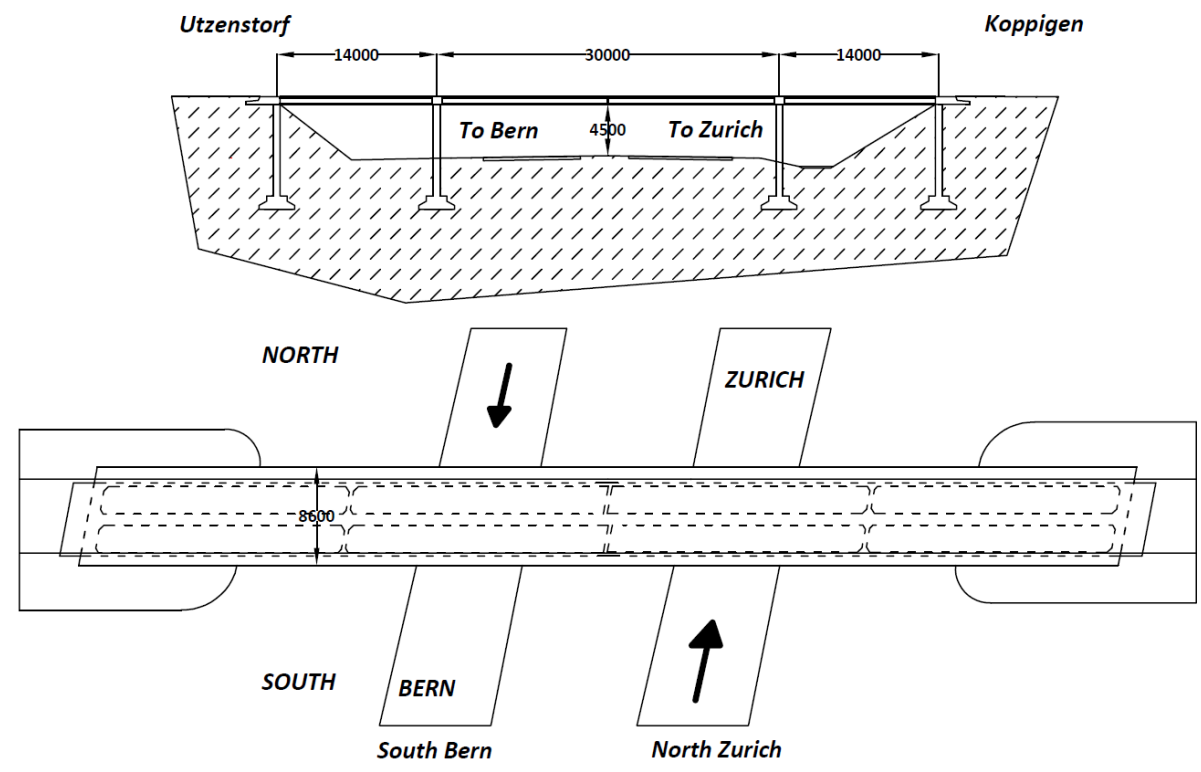

Figure 4.9: Schematic of the Z24 bridge.

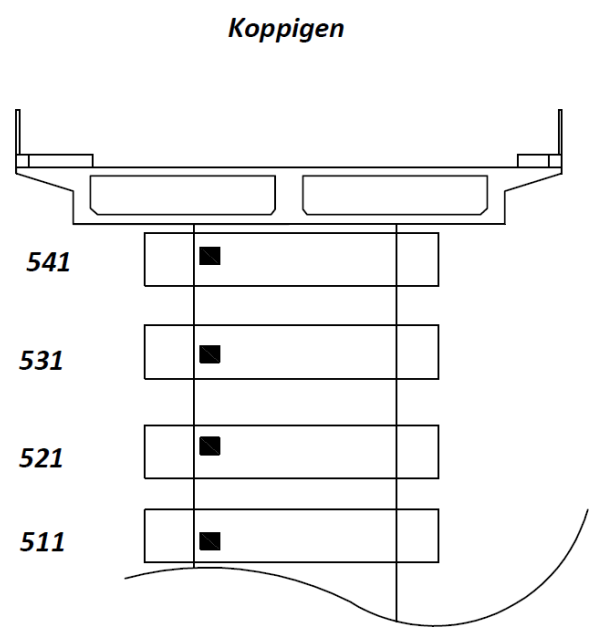

Damaged Pier
Utzenstorf

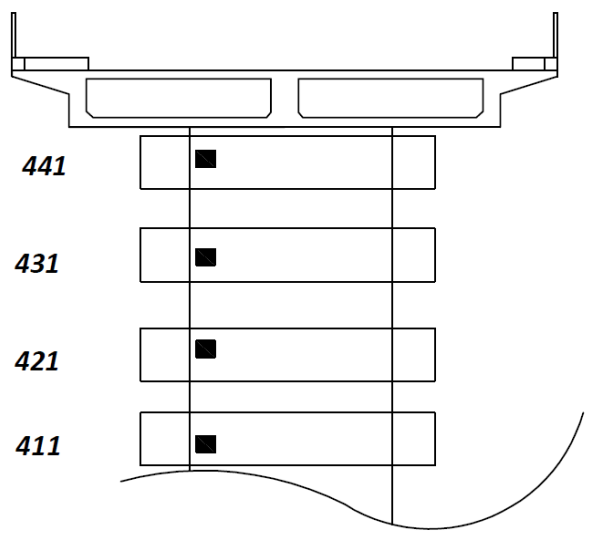

Undamaged Pier

Figure 4.10: Schematic of the Z24 bridge piers and the selected sensors used in this study. 


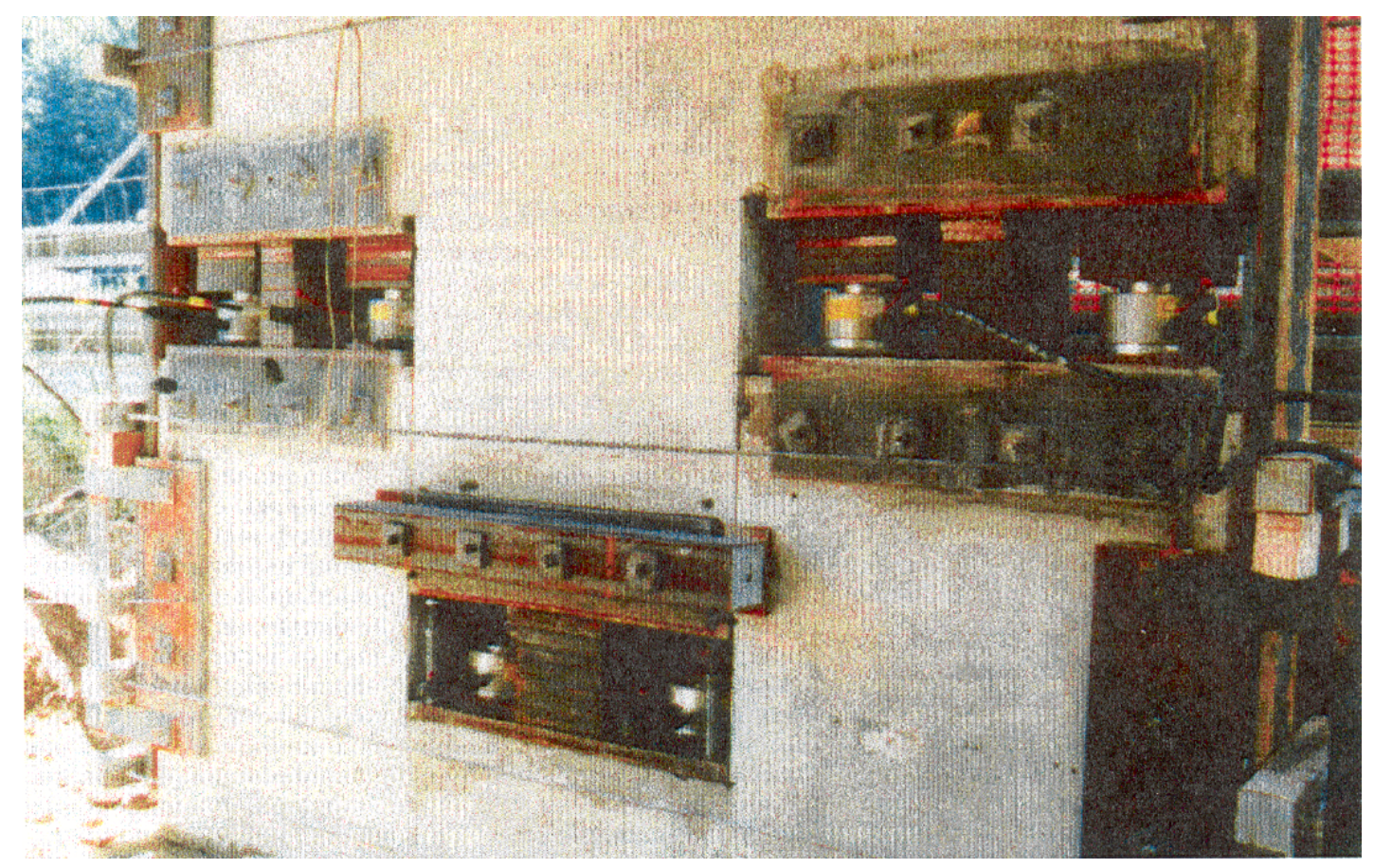

Figure 4.11: Lowering of the piers by cutting concrete [106].

\subsubsection{Identification results}

The acceleration measurement and corresponding Fourier spectrum are presented for both undamaged and damaged cases, as shown in Fig. 4.12. The damaged pier is localized using the proposed algorithm. Various cases of limited sensors were used to illustrate the performance of the proposed algorithm. There are four sensors each on both of piers (a total of eight sensors), and for limited sensors case, the number of sensors were reduced to six, and four, respectively. $\overline{\Delta E}$ of each sensor is used to present the damage localization feature for various cases.

Three different damage localization scenarios of pier settlement are considered, namely, $20 \mathrm{~mm}, 40 \mathrm{~mm}$ and $95 \mathrm{~mm}$ settlement to include the severity of damage in the analysis. As shown in Fig. 4.13 for $20 \mathrm{~mm}$ pier settlement, it can be observed that $\Delta E_{a i}$ of the 
damaged pier is consistently higher than the $\overline{\Delta E}$ in all limited sensor cases. Similar analysis is conducted for $40 \mathrm{~mm}$ and $95 \mathrm{~mm}$ lowering of pier as shown in Fig. 4.14 and Fig. 4.15, respectively. The results show that the proposed method is able to detect the damage with various severities as well as identify the location of damage using a suite of limited sensor cases across various levels of damage.
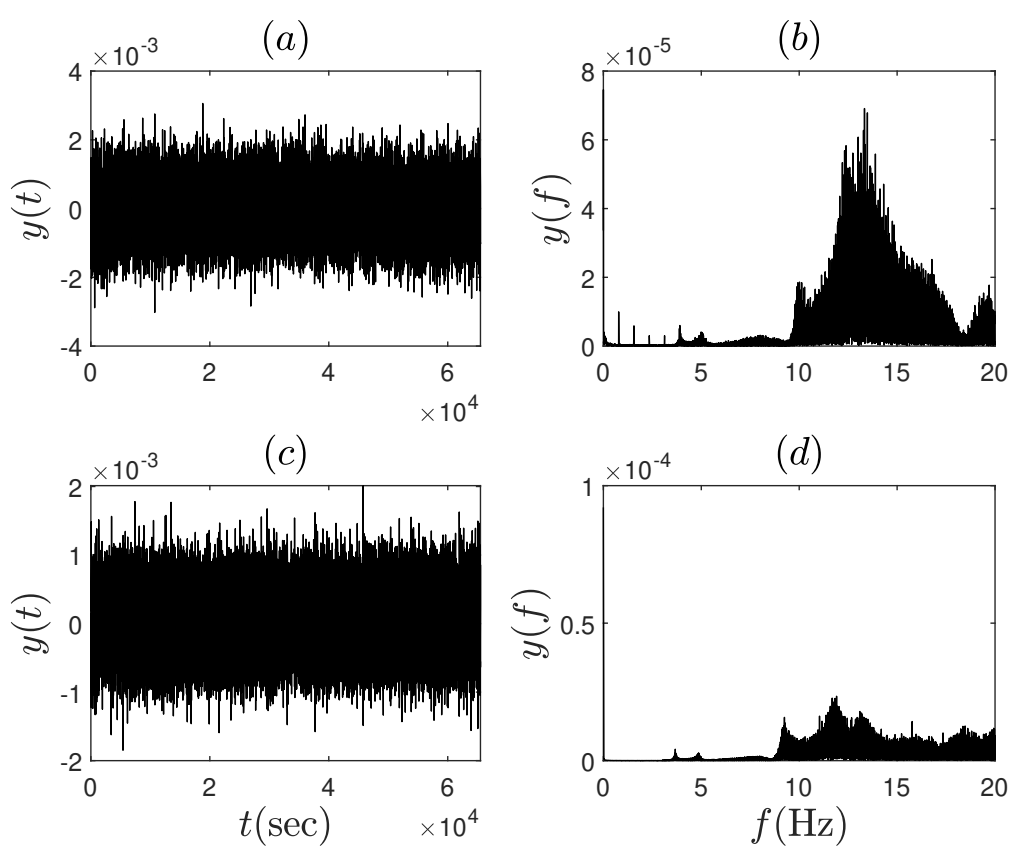

Figure 4.12: The data of the Z24 bridge: (a) time history and (b) Fourier spectrum of the response of the undamaged pier (sensor-421), (c) time history and (d) Fourier spectrum of the damaged pier (sensor-521). 
(a)

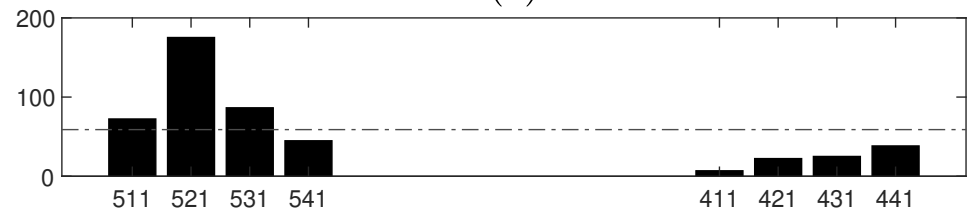

(b)

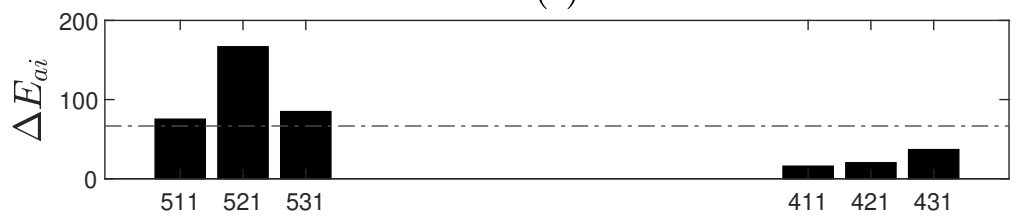

$(c)$

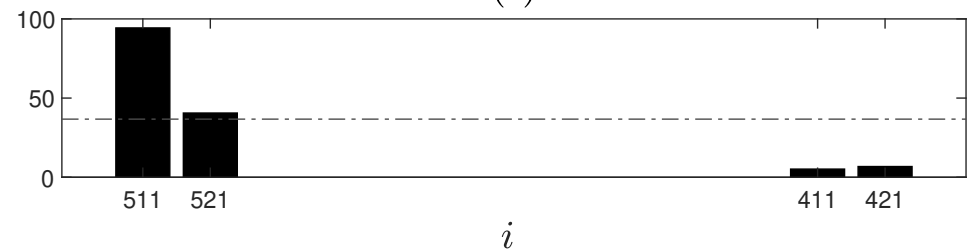

Figure 4.13: $\Delta E_{a i}$ for $20 \mathrm{~mm}$ lowering of various sensor cases using, (a) 8 sensors, (b) 6 sensors, (c) 4 sensors.

(a)

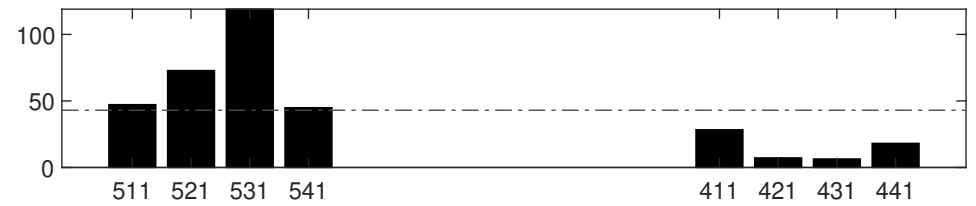

(b)

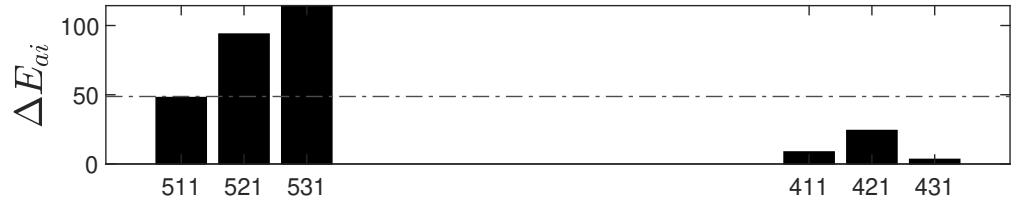

(c)

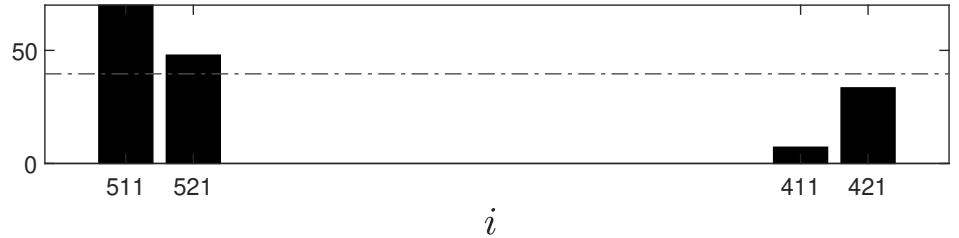

Figure 4.14: $\Delta E_{a i}$ for $40 \mathrm{~mm}$ lowering of various sensor cases using, (a) 8 sensors, (b) 6 sensors, (c) 4 sensors. 
(a)

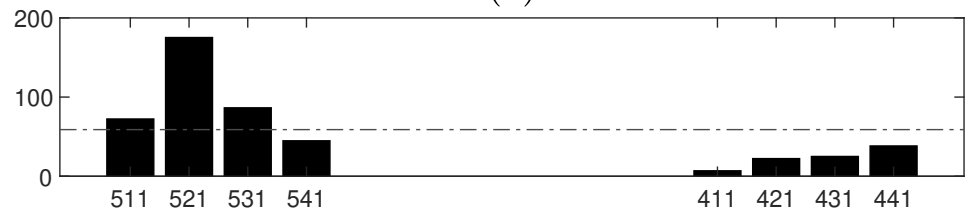

(b)

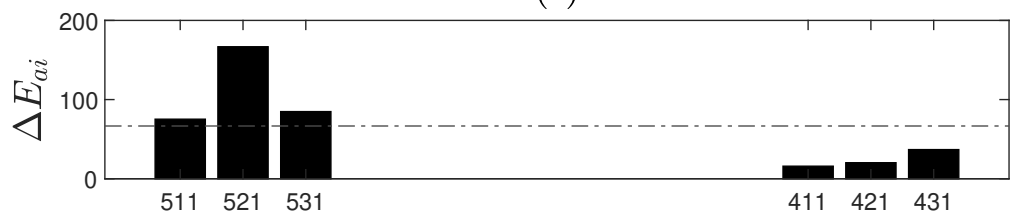

$(c)$

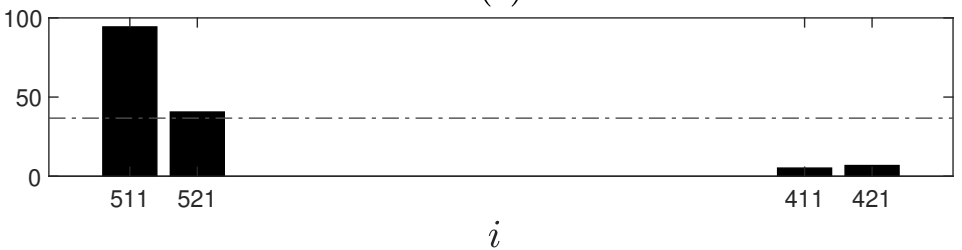

Figure 4.15: $\Delta E_{a i}$ for $95 \mathrm{~mm}$ lowering of various sensor cases using, (a) 8 sensors, (b) 6 sensors, (c) 4 sensors.

The variability for $95 \mathrm{~mm}$ pier settlement in $\Delta E_{a i}$ for each mode of every sensor is presented as box plots and for various limited sensors damage cases, as shown in Figs. $4.16,4.17$, and 4.18 , respectively. It can be observed that along with mean of $\Delta E_{a i}$ for each sensor, the variability in the modal $\Delta E_{a i}$ for the damaged cases are larger than $\overline{\Delta E}$, indicating clear delineation of the damage location at the damaged pier. It can be observed that high variation is in the sensors from the damaged pier (i.e., 511, 521, 531 and 541) and low variation in the undamaged pier (i.e., 411, 421, 431, and 441). Moreover, the damage index of the damaged pier is always higher than the threshold value irrespective of the number of limited sensors, indicating the efficacy of the proposed method to identify the damage location. 


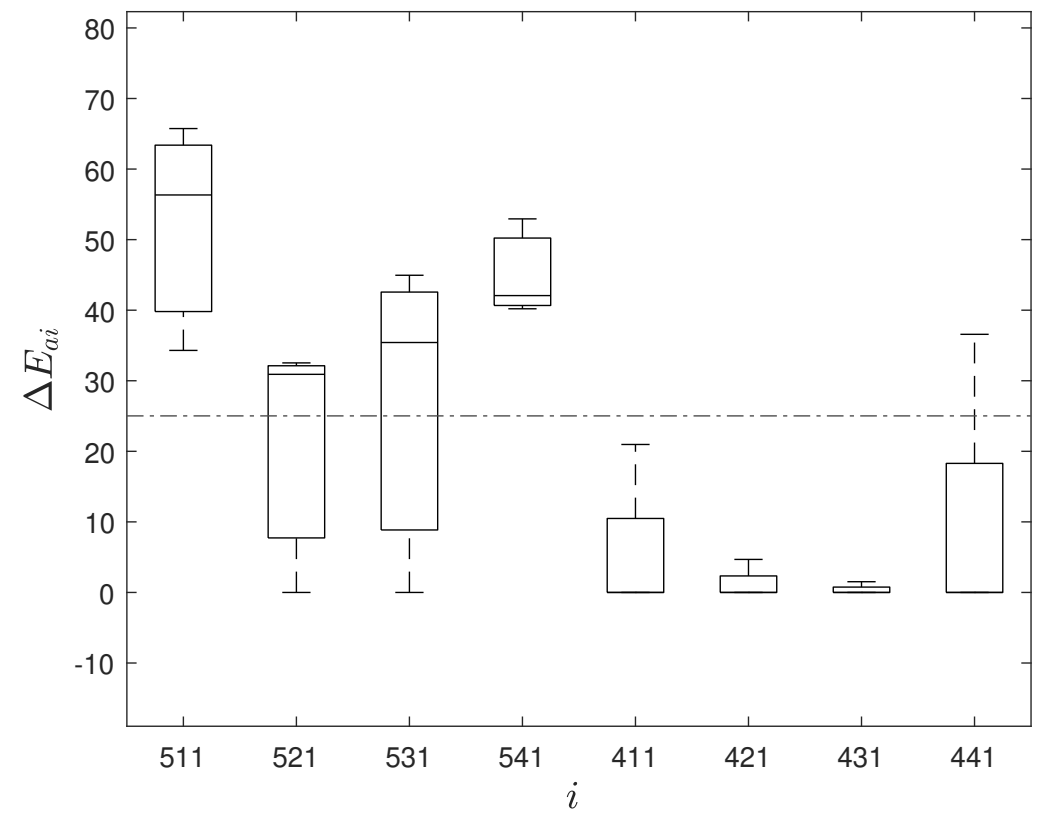

Figure 4.16: $\Delta E_{a i}$ of IMFs of the Z24 bridge using 8 sensors.

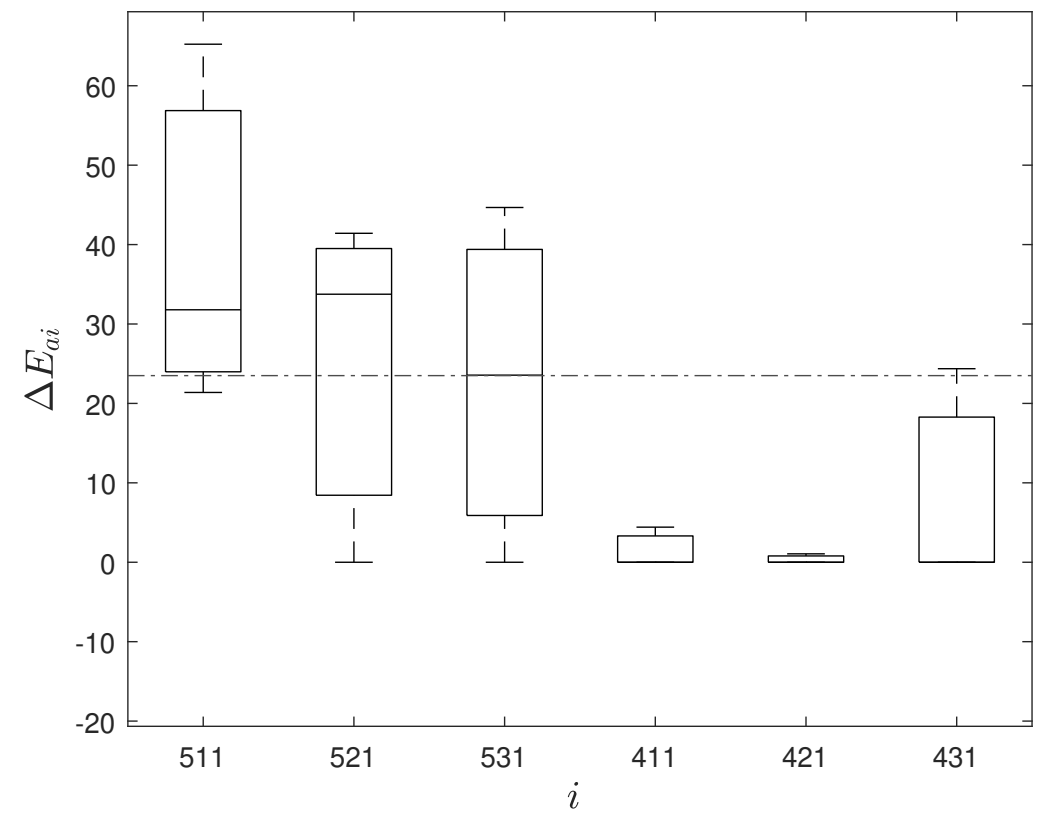

Figure 4.17: $\Delta E_{a i}$ of IMFs of the Z24 bridge using 6 sensors. 


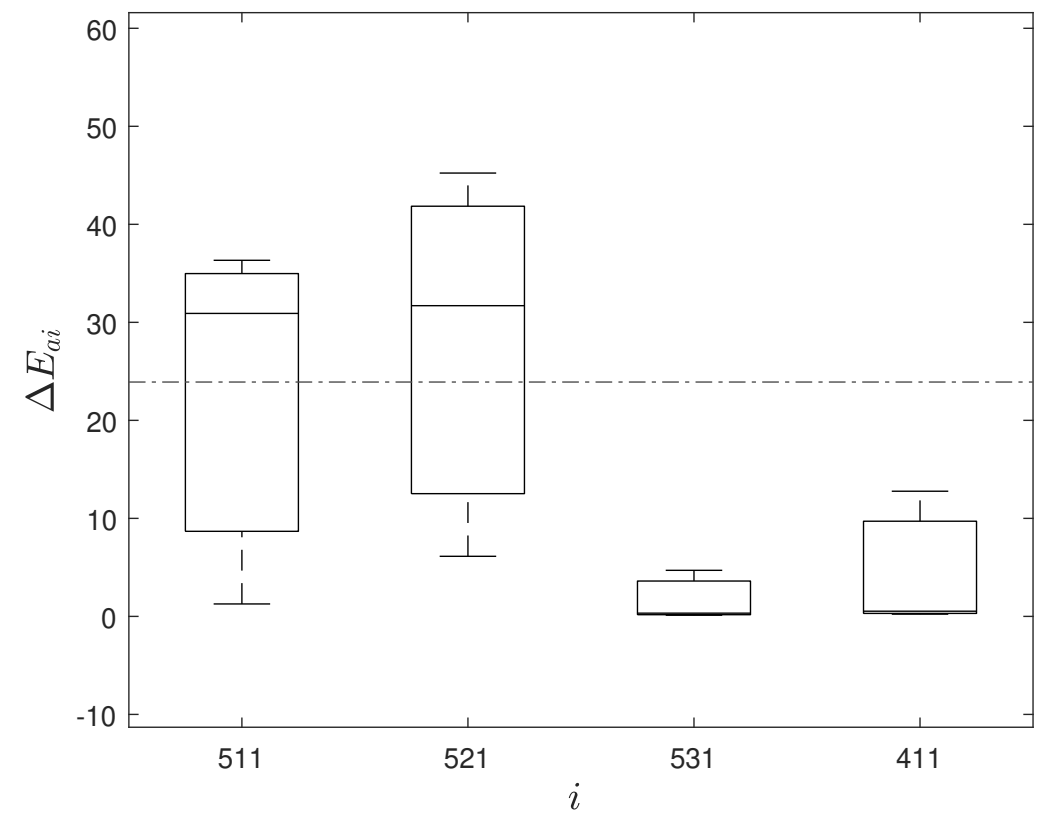

Figure 4.18: $\Delta E_{a i}$ of IMFs of the Z24 bridge using 4 sensors.

\subsection{Summary}

In this chapter, a novel damage localization is proposed using a damage index obtained from MEMD. MEMD is used to decompose multi-sensor data into their mono-components. The mono-component modal responses are further used to evaluate the modal energy to derive the damage localization feature using limited sensors. The proposed method is demonstrated using a suite of numerical studies and the benchmark data of Z24 bridge. It is concluded that the proposed method works well on all the studies and is effective in localizing the damage. The limited measurement aspect of damage localization is explored by selecting a fewer number of sensors and it is shown that with limited measurements, the proposed method is as effective as total number of measurements equals to the degree of freedom of the model. The results show the capability of the proposed method in identifying 
as minimal as $2 \%$ change in global modal parameters of structures, outperforming the existing TF methods to delineate the minor global damage. 


\section{Chapter 5}

\section{Multiclass Damage Identification}

\section{using One Dimensional Convolutional}

\section{Neural Networks}

The objective of this chapter is to create an autonomous damage detection framework with minimal to no user intervention. Unlike TF methods or ML techniques, Deep learning (DL) based method is proposed to create a self-learning data-driven environment without the need of any feature extraction for multi-class VDD. 1D CNN is explored to model limited data set for damage detection and localization by data augmentation using windowed approach for every class of damage. The limitation of 1D CNN to not effectively learn with limited datasets is improved by augmenting the data by a novel windowed-voting approach. 


\subsection{Introduction}

Data-driven damage diagnosis is a critical component of infrastructure asset management [103]. Although there is a plethora of research on parametric methods based on time-frequency (TF) decomposition techniques [124],[7],[21],[70], non-parametric methods [94],[134],[2] have shown significant promises in data-driven SHM methods. Parametric methods include extracting dynamic parameters such as modal parameters, while inferring the change in these parameters to detect any possible changes in the structures. On the other hand, non-parametric methods include extracting parameters that are estimated based on the computational models and the parameters are mathematically derived in a statistical sense.

Structural damage identification can be considered as a pattern recognition-based nonparametric problem, which is divided into three stages, namely, data acquisition, feature extraction, and feature classification. With proliferation of various machine learning (ML) algorithms, the SHM community has prominently used various supervised learning algorithms [63],[15]. In [46], the authors explained the interface between nondestructive evaluation and machine-learning-based SHM for damage detection. The study highlighted the need for a combination of compressive sensing based sparse methodology with datadriven machine learning methods. [125] presented a critical review of field monitoring of high-rise structures. The study reviewed techniques for comfort assessment, seismic and wind effects, and environmental effect on monitoring of super-tall structures. Recently, the SHM community has explored both vibration and image data for structural damage identification and localization. 
With advancements in artificial intelligence, image-based SHM has garnered as an inexpensive way to monitor large scale structures using Convolutional Neural Networks (CNNs). While image-based 2D CNN techniques remain a popular method for SHM [77],[32],[54],[119], they involve significant complexity in obtaining a large amount of labelled data, pre-processing and classifying the images. As a solution, researchers have studied algorithms that directly operate on the sequential data such as vibration data. [56] proposed sparse coding as a feature extraction method for unlabeled acceleration measurements obtained from wireless sensors. The damage classification was carried out using a CNN, and the results were compared with logistic regression and decision trees. A threespan bridge was considered to evaluate the efficacy of the proposed method, and it was shown that sparse coding-CNN based method outperforms other methods with an accuracy of $98 \%$. [55] conducted a simulation study on a steel gusset plate connection by varying the size and location of the damage. A CNN was used to classify damaged signals, and the proposed method achieved a testing error of $2 \%$ and showed robustness against environmental noise. [44] explored the applicability of dynamic features such as mode shapes, frequency response functions, and natural frequencies as damage indicators under varying temperatures. The authors used a combination of couple sparse coding and deep neural network as an ensemble method for damage detection and localization. The proposed method was validated on a numerical truss bridge and experimental I-40 benchmark dataset. [20] proposed a CNN-based anomaly detection using acceleration measurements by converting them into grayscale images. The authors used several anomaly parameters such as missing, minor, outlier, square, drift, and trend data points to train the datasets 
using a stacked autoencoder architecture. [114] proposed deep convolutional denoising autoencoders for structural damage detection. The proposed method extracted damage features from field measurements under environmental noise.

Recently, 1D-CNN have shown promising results in capturing the temporal information and damage detection using vibration data. [4] introduced 1D CNN for real-time vibrationbased damage detection. The authors trained 1D CNN on a vibration signal database obtained from a truss, named Qatar Grandstand, by damaging each joint and keeping the other joints undamaged. The proposed model was trained individually on each joint, and near-perfect classification accuracy was proposed. However, the proposed method was not tested for a multiclass damage scenario. [149] utilized the computational powers of 1D CNN to detect changes in structural parameters such as stiffness and mass. Three different structural components were used for data acquisition and model validation, namely, Tshaped steel beam, short and long steel girder bridge, and a mean classification accuracy of $98 \%$ was achieved. [99] showed the applicability of 1D CNN with autoencoders for anomaly detection under data compression. The proposed algorithm was validated using a long-span suspension bridge with an accuracy of $97.53 \%$.

A recent study by [16] explored the concept of transfer learning in vibration measurements. The authors used a four-story IASC-ASCE SHM model for numerical training, and the proposed model was tested on experimental studies using IASC-ASCE SHM benchmark building and the Qatar University Grandstand Simulator with an accuracy of 90100\%. Recently, [115] showed the applicability of 1D CNN for damage detection in the structural frames. Experimental validation was performed on a 2D-steel frame with dif- 
ferent damage location and severity of the damage. The method was shown to identify different damage scenarios and the false-positive rate was also evaluated and found to be well within the acceptable limits. Furthermore, [84] conducted a study by integrating traditional TF methods with the capability of neural networks. The authors used transmissibility function-based 1D CNN to effectively identify damage at the ASCE SHM benchmark structure. The proposed method was compared with the time-series and fast Fourier transform-based frequency-domain information, where the TF signals exhibited more significant damage-sensitive features. Overall, 1D CNN primarily exhibited superior performance over artificial neural networks (ANNs) in the context of computation efficiency and noise insensitivity for big data [74]. Recently, [19] evaluated a combination of finite element method (FE) and 1D CNN for localizing damage for a jacket-type offshore structure. However, the study lacks in two aspects; first, the data was generated synthetically using a finite element model, which might not resemble the actual real-world data with operational and environmental noise contamination. Second, the damage was induced artificially using the FE model that was clearly distinguishable from an undamaged structure that does not concur with the real-world data.

The proposed research explores the existing challenges of multiclass damage localization using 1D CNN. Unlike the simulated data, the real-world data is limited and noisecontaminated, where multiclass damage localization becomes a significant challenge. In this chapter, 1D CNN is explored for multiclass damage localization with varying damage severity under different damage scenarios. The issue of the limited dataset is solved by augmenting the data using windowing the acceleration measurements, and the classifica- 
tion results are improved using a novel voting approach on the prediction class. The study presents the benefits of using fast and computationally inexpensive 1D CNN with only one hidden layer for limited operational data for damage classification in a full-scale bridge.

The chapter is structured as follows. A brief introduction of the structural damage identification, its need, and a literature review based on 1D CNN techniques are presented in Section 5.1. Section 5.2 explains the theoretical background of the proposed algorithm, along with the selected architecture of 1D CNN. Section 5.3 presents the capability of the proposed algorithms to identify and localize multiclass damage, the importance of hyperparameter tuning and various metrics to show the damage parameters of the structures. A brief summary of this chapter is presented in Section 5.4.

\subsection{Proposed Methodology}

\subsubsection{Background}

Convolutional Neural Networks (CNNs) are a type of feedforward neural network model that is designed to approximate some function $y=f(x ; \theta)$. For classification, the model maps an input $x$ to a category (class) $y$. The parameters $\theta$ are learned to best fit a given training dataset by a gradient descent optimization algorithm [50]. The most common type of CNNs are 2D CNNs that are used in the field of computer vision for tasks such as image classification, where the inputs $x$ are matrices (2D shaped) representing images. 1D CNNs are a simpler variant of CNNs, where the inputs $x$ are vectors (1D shaped), typically representing a time-series. They are commonly used for tasks involving time 
signal processing such as speech recognition (Kiranyaz et al. 2019). 1D CNNs became popular in SHM since the last few years due to its computational simplicity in comparison to its parent family of $2 \mathrm{D}$ and $3 \mathrm{D}$ CNNs as it requires simple array application and a shallow network.

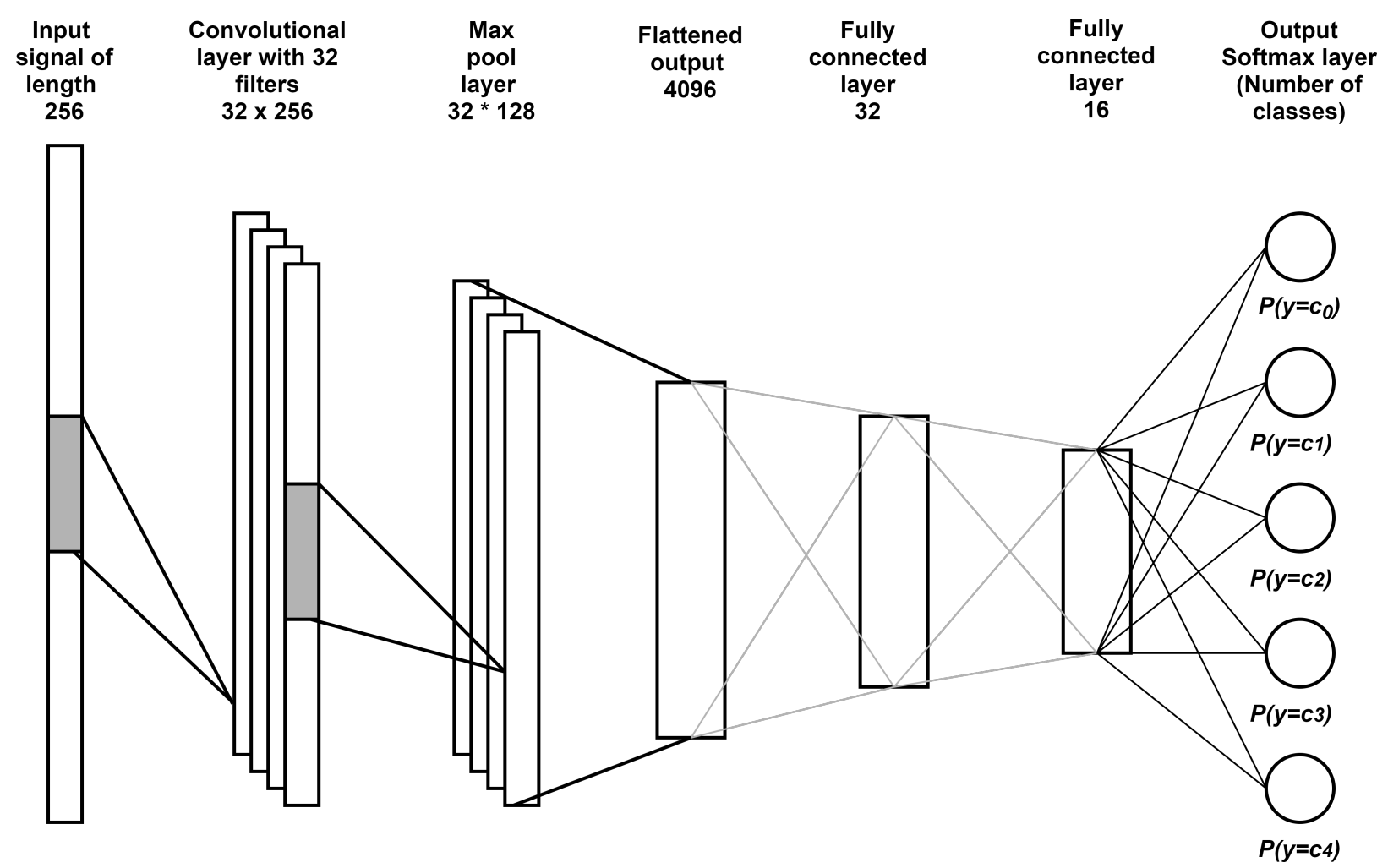

Figure 5.1: 1D CNN architecture used in this study.

A typical 1D CNN architecture used in this study is shown in Fig. 5.1. It consists of an input layer (time-series), multiple alternating convolutional and pooling layers, and one or more fully connected layers at the end. An input time-series $x$ presented to the input layer is transformed by the forward pass through the hidden layers and the output softmax layer produces the class label $y$. When the number of hidden layers is high, this architecture is referred to as a deep convolutional neural network. The convolutional layer is the core building block of a CNN. The parameters of each convolutional layer consist of 
a set of learnable kernels, which are defined by a kernel length $(m)$. Convolutional layers have a reduced number of parameters in comparison to fully connected layers as a single kernel share the weights for spatial locations in the input. The convolution process can be expressed as Eq. 5.1 [50]:

$$
y(n)=f(x(n) \otimes h(m))
$$

where $x(n)$ is the input vector of length $n$ and $h(m)$ is kernel of length $m$. The symbol $\otimes$ denotes the traditional 1D convolution between two signals as defined below,

$$
x(n) \otimes h(m)=\sum_{k=0}^{n} x(k) h(n-k)
$$

In Eq. 5.1, the function $f$ is called an activation function, which is typically a nonlinear transformation on the traditional 1D convolution. Non-linear activations enable the network to learn complex mappings between the input signal and the class labels. In this study, Rectified Linear Unit (ReLU) is used as the activation function, which effectively removes negative values from an activation map by setting them to zero. A pooling layer is added after the convolution layer to sub-sample the convolution output. The pooling operation reduces the dimensionality of a given mapping while highlighting the prominent feature and it also helps to reduce overfitting. Max pooling refers to selecting the maximum value in a window that slides over the input map. In Fig. 5.1, the max pooling layer has reduced the size of each convolution output size by a factor of two. For the output layer, the choice of activation function depends on the type of output. For classification problems, 
SoftMax activations are preferred. SoftMax function for a n-class problem (representing $n$ probabilities of input belonging to each of n-classes) is shown in Eq. 5.3.

$$
P(\text { class }=j \mid z)=\frac{e^{z_{j}}}{\sum_{k=1}^{n} e^{z_{k}}}
$$

where $z_{j}$ is the input to the softmax node $j$ from the previous layer.

\subsubsection{Multiclass damage detection using windowed-voted 1D CNN}

A method based on a 1D CNN neural network model is proposed to classify the vibration measurement into multiple damage level classes (i.e., multi-class classification). First, each acceleration signal is scaled to fit a standard normal distribution (subtract the mean of sample values and divide by the standard deviation). The scaling improves the convergence rate of models trained on the dataset and prevents any outlier from dominating the input [67]. In order to train a neural network to achieve high test accuracy, a large amount of training data is required. Due to the scarcity of vibration-based multiclass data for civil infrastructure, it is critical to augment the training dataset. In the proposed method, the dataset of raw acceleration signals is augmented by extracting windows of samples from the original signals, as shown in Fig. 5.2. Each extracted window is assigned the same damage level label as the original time-series. A new dataset is formed by taking the extracted windows and their labels as the training instances. In addition to increasing the number of training instances, this windowing technique also reduces the data dimensionality (shorter input signals), which allows training machine learning models with less over-fitting. 


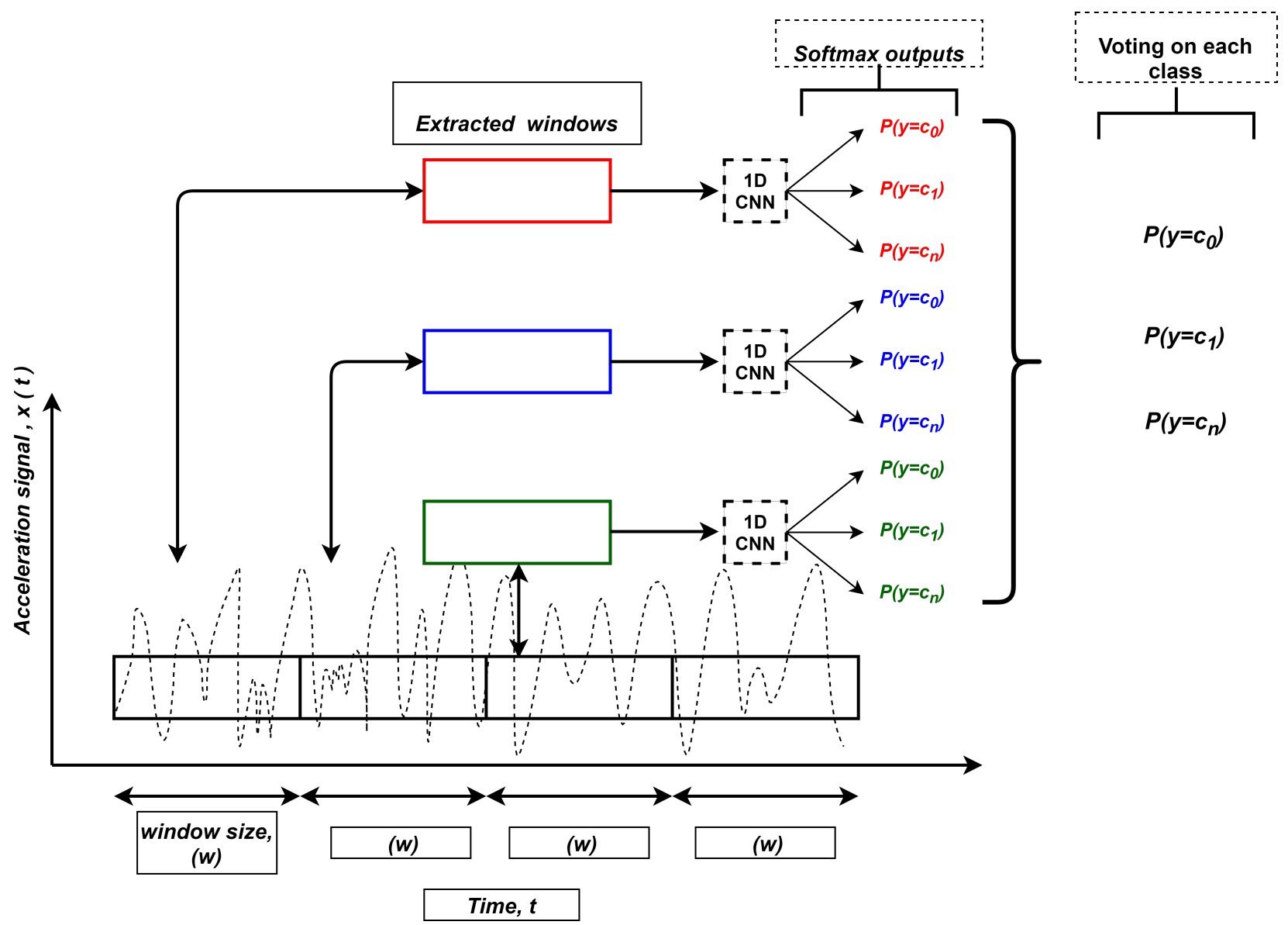

Figure 5.2: Extracting data sequences of windows from the vibration data using 1D CNN architecture.

The dataset is then split into training, validation and test sets. A 1D CNN model is trained on the dataset using a standard gradient descent optimizer. Hyper-parameters of the 1D CNN model include the number of layers and number of nodes in each layer, activation function and the kernel size in convolutional layers. Additionally, the length of the extracted windows is also considered as a hyper-parameter. Finding the optimal hyper-parameters (also known as hyper-parameter tuning) is done by a random search over the parameter space and selecting the configuration that yields the high accuracy on the validation set [22]. 
In order to classify a new acceleration signal at test time, windows are extracted as before and fed into the trained 1D CNN model, which outputs a set of classification probabilities for each window. The predicted set of classification probabilities $P_{p}\left(y_{c}\right)$ for a full acceleration measurement is obtained by summing the class probabilities of all the window sequences in a single time-series. The class with the maximum probability is the predicted damage level classification of the series, as shown in Fig. 5.2. It may be noted that this is equivalent to voting on the classification probabilities of individual window sequences to arrive at the prediction based on the entire time-series. It is observed that the voting process improves the prediction accuracy and other evaluation metrics in the time-series. The proposed machine learning pipeline for the multiclass damage classification problem is shown in Fig. 5.3.

\subsubsection{Performance criteria}

In machine learning, a number of performance metrics are used to evaluate the efficacy of the computational model. A brief description of metrics used to evaluate classification models in the context of SHM is provided below. The confusion matrix is a tabulation of classifications made by a model, typically with the actual class in rows and predicted class in columns. Table 5.1 shows the confusion matrix for a multi-class classification problem with three classes $(\alpha, \beta$, and $\gamma)$. As shown, $T P_{\alpha}$ is the number of true positive samples in class $\alpha$, i.e., the number of samples that are correctly classified from class $\alpha$, and $E_{\alpha \beta}$ is the samples from class $\alpha$ that are incorrectly classified as class $\beta$, i.e., misclassified samples.

Therefore, the false negative in the $\alpha$ class $\left(F N_{\alpha}\right)$ is the sum of $E_{\alpha \beta}$ and $E_{\alpha \gamma}\left(F N_{\alpha}=\right.$ 
$\left.E_{\alpha \beta}+E_{\alpha \gamma}\right)$ which indicate the sum of all class $\alpha$ samples that were incorrectly classified as class $\beta$ or $\gamma$. Simply, FN of any class can be calculated by adding the errors in that class/column. On the other hand, the false positive for any predicted class which is located in a row represents the sum of all errors in that specific row. For example, the false positive in class $\alpha, F P_{\alpha}$ is calculated as follows, $F P_{\alpha}=E_{\beta \alpha}+E_{\gamma \alpha}$. Therefore, for a $k * k$ confusion matrix there are $k$ correct classifications and $k^{2}-k$ possible errors [123].
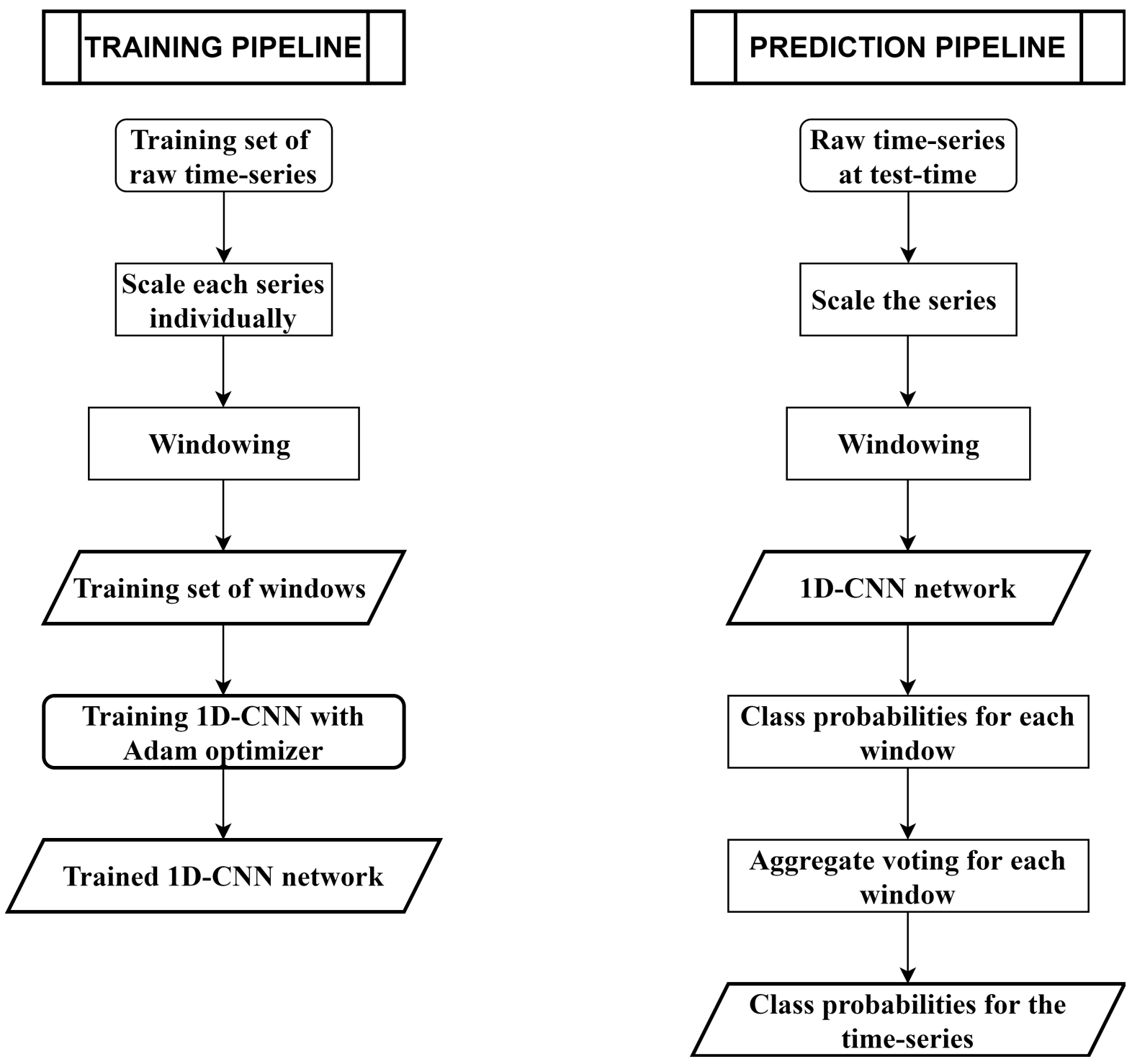

Figure 5.3: Data pipelines for training the proposed 1D CNN network and obtaining predictions for a given time-series. 
Table 5.1: Confusion matrix for a multiclass problem.

\begin{tabular}{c|ccc}
\multicolumn{4}{c}{ True class } \\
\hline \multirow{4}{*}{ Predicted Class } & $T P_{\alpha}$ & $E_{\beta \alpha}$ & $E_{\gamma \alpha}$ \\
& $E_{\alpha \beta}$ & $T P_{\beta}$ & $E_{\gamma \beta}$ \\
& $E_{\alpha \gamma}$ & $E_{\beta \gamma}$ & $T P_{\gamma}$ \\
\hline
\end{tabular}

There are various metrics that are derived from confusion matrix and are presented in Table 5.2. In the context of SHM and multiclass damage detection, ROC-AUC, Accuracy, FNR and F1 score are used to evaluate the performance of the proposed method. Accuracy is a primary performance metric used to evaluate the ability of a model to correctly classify the data samples into various class labels. Another important metric that has not been discussed in the literature is FNR. In the SHM context, it is critical that a damage detector have a low FNR, as a false negative corresponds to the potentially catastrophic case of a damaged signal being classified as an undamaged signal. Furthermore, a damage detector must have high values for accuracy, and F1 score. Additionally, two curves are used to evaluate the trade-off between performance metrics. The receiver operating characteristic (ROC) curve shows the trade-off between TPR and FPR as the decision threshold of the classifier varies. The precision-recall (PR) curve shows the trade-off between precision and recall as the decision threshold of the classifier varies. The area under the curves (ROCAUC and PR-AUC) is a summary metric that reflects the level of possible trade-off. Both curves are useful for an engineer to find a suitable decision threshold. 
Table 5.2: Description of various performance metrics.

\begin{tabular}{|c|c|c|}
\hline Metric & Expression & Remarks \\
\hline \hline ROC-AUC & TPR Vs FPR & Degree of separability between classes \\
\hline Accuracy (A) & $\frac{T P+T N}{T P+F N+F P+T N}$ & Less useful for heavily imbalanced data \\
\hline Precision (P) & $\frac{T P}{T P+F P}$ & Positive predicted value \\
\hline Recall (R) & $\frac{T P}{T P+F N}$ & True positive rate or sensitivity \\
\hline False Positive Rate (FPR) & $\frac{F P}{T N+F P}$ & False alarm when there is no damage \\
\hline False Negative Rate (FNR) & $\frac{F N}{T P+F N}$ & No alarm for actual damage \\
\hline
\end{tabular}

\subsubsection{Damage localization}

Damage localization for multi-class problems is evaluated using Algorithm 4. The entire structure is modeled as one experiment rather than modeling each sensor separately as in [4] and prediction probabilities are acquired for each sensor location. However, as there are multiple sensors covering the entire structure, different structural components are collectively used to localize damage using a limited number of sensors for each component. The damage is confirmed if the true predicted probability class is equal to allocated class label for all cumulative windowed series for each sensor location and $P_{p}\left(y_{c}\right)$ is greater than the threshold. 


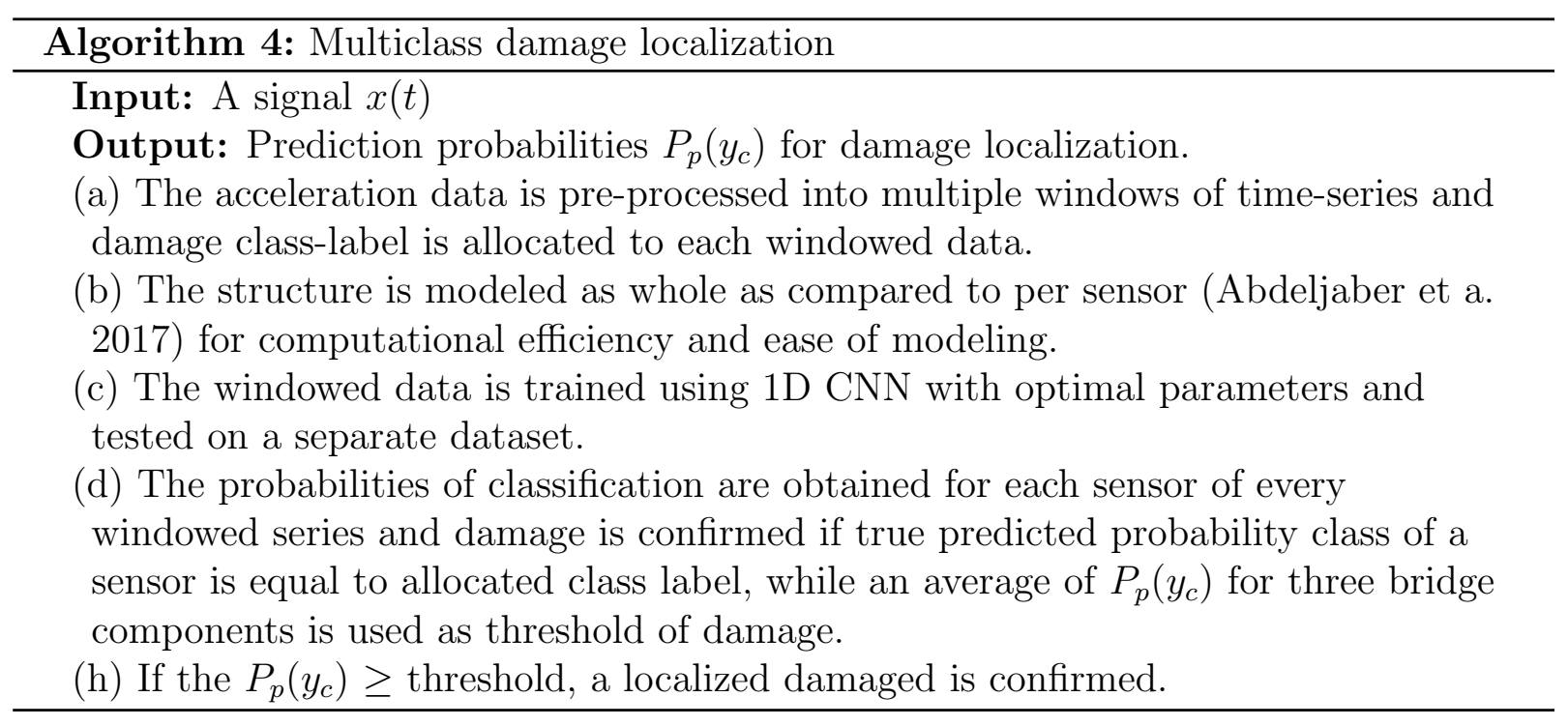

\subsection{Full-scale Study}

\subsubsection{Details of Z24 Bridge}

Damage detection, where classification is more than two classes, is considered as a multiclass problem. In this study, two types of damage cases are used, namely, rupture of tendons, and pier settlement of a full-scale bridge, namely, Z24 Bridge. All the damage classes have multiple damage levels. Z24 bridge benchmark data [91] is used to evaluate the performance of the proposed method for multiclass damage detection. The bridge was excited by two shakers, one at the mid-span of the bridge and another at a side-span. Because of the size of the bridge, response was measured in nine setups of up to 15 sensors each, with three accelerometers and the two force sensors common in all setups. The details of the data are explained in section 4.4. 


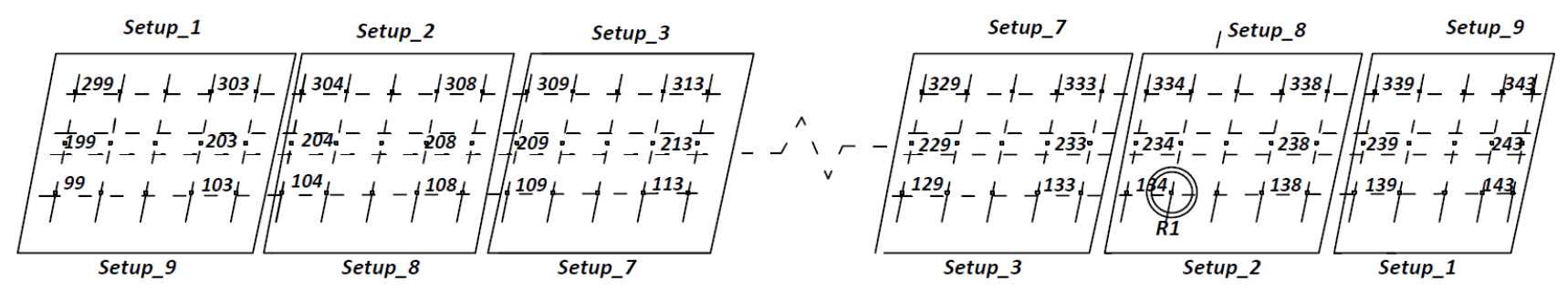

Figure 5.4: Sensor placement for data acquisition.

The data was acquired by performing various progressive damage scenarios during the demolition period. For the brevity of this study, only two different damage scenarios are considered: rupture of tendons, and pier settlement. It may be noted that each damage scenario have different classes of damage, and they were chosen to evaluate the performance of the proposed method to classify various multiclass damage cases. For example, rupture of tendons have three levels, and pier settlements have four levels, and together they made a case of two separate damage classes. For detailed explanation of how the damages were induced in the bridge, the readers are suggested to refer [106]. The reference, undamaged condition is considered as class-zero for all the cases and the other damages were assigned classes starting from 1 to $n$ depending upon the level of damage, as shown in Table 5.3. For example, in the case of rupture of tendons, the damage was induced at first, rupture of two tendons, and second, rupture of four tendons, third, rupture of six tendons, thereby creating three classes of damages for rupture of tendons. Similarly, there are four classes for pier settlement. 
Table 5.3: Description of the multiclass damage scenarios and the class labels.

\begin{tabular}{ccc} 
Problem & Damage scenario & Class label \\
\hline \hline 0 & Undamaged & 0 \\
\hline \multirow{2}{*}{1} & Rupture of 2 tendons & 1 \\
& Rupture of 4 tendons & 2 \\
& Rupture of 6 tendons & 3 \\
\hline \multirow{2}{*}{2} & Lowering of pier, $20 \mathrm{~mm}$ & 1 \\
& Lowering of pier, $40 \mathrm{~mm}$ & 2 \\
& Lowering of pier, $80 \mathrm{~mm}$ & 3 \\
& Lowering of pier, $95 \mathrm{~mm}$ & 4 \\
\hline
\end{tabular}

\subsubsection{Hyper-parameters of the 1D CNN model}

An introduction to the full-scale study based on various damage scenarios of the Z24 Bridge is provided first, followed by the hyper-parameters used for the computational models. The evaluation metrics based on the proposed method are described later with a comparison between window-voted and non-voted results. In this study, a range of hyper-parameters are selected first and tuned using random search algorithm to achieve a set of hyperparameter that provides the optimal accuracy. The range of hyper-parameters used for 1DCNN are presented in Table 5.4. For example, window size is adopted within a range of 64-512 samples. Window size is the only external parameter and is decided by the user. Thus, a sensitivity analysis is performed to understand the behavior of the performance evaluation metrics $\left(P_{m}\right)$ under different window sizes $(w)$. Two different metrics, accuracy and FN, are used for sensitivity analysis as they represent overall accuracy of the model and false-negative alarm critical for civil infrastructure. 
Table 5.4: Hyper-parameters used in 1D CNN for tuning by random search algorithm.

\begin{tabular}{ll}
\hline Parameter & Values \\
\hline \hline Window size & $64,128,160,256,512$ \\
No. of hidden convolutional layers & $1-6$ \\
No. of filters & $1024,512,256,128,64,32$ \\
No. of fully connected layers & 1 or 2 layers with 16 and 32 nodes \\
Learning rate & $0.0003,0.001,0.01$ \\
Batch size & $64,256,512$ \\
Kernel size & $8,16,32,64$ \\
\hline
\end{tabular}

The optimal hyper-parameters of this dataset are obtained after tuning and are presented for all the models in Table 5.5. An analysis is performed to understand the effect of $w$ versus $P_{m}$. The results are shown for various damage cases in Fig. 5.5. For example, Fig. 5.5 (a-b) shows that the optimal performance is achieved at $w=256$, with highest ROC and accuracy, and lowest false-negative. Although, the FNR remains consistent after $w=512$ and other metrics are at their peak, however, due to larger $w$, the data size reduces per damage class and it leads to over-fitting of the data.

\subsubsection{Random initialization of weights}

Deep learning algorithms are iterative and require the user to specify value of initial weights of neurons to initiate the iteration and its optimization. In practice, all weights in the model are randomly drawn from a Gaussian or uniform distribution. The choice of Gaussian or uniform distribution does not seem to matter much but has not been exhaustively studied [50]. However, the scale (low or high magnitude) have a large effect on both 
Table 5.5: Optimal configuration of the hyper-parameters of the selected 1D CNN.

\begin{tabular}{ll}
\hline Parameter & Values \\
\hline \hline Window size & 256 \\
No. of hidden convolutional layers & 1 \\
No. of filters & 32 \\
No. of fully connected layers & 2 with 32 and 16 nodes, respectively \\
Learning rate & 0.0003 \\
Batch size & 256 \\
Kernel size & 16 \\
\hline
\end{tabular}

the outcome and optimization procedure. In this study, random initialization with early stopping criteria is used and Adam optimizer [73] is used with dropout in each layer for regularization. After acquiring the optimal tuned parameters, a parametric study is conducted to understand variance in the metrics of 1D CNN model for random initialization of weights. The metrics used for evaluating random initialization of weights are ROC-AUC, accuracy, FNR, and F1 score and are shown in Table 5.6. It can be observed that for pier settlement, the mean $(\mu)$ of ROC-AUC is 0.97 with an accuracy of 0.85 . The FNR is 0.15 and the standard deviation $(\sigma)$ for all the trials is at its minimal of $1 \%$. Similarly, for rupture of tendons, the ROC-AUC is 0.92 with an accuracy of 0.67 and FNR of 0.33 with minimal $\sigma$ of $2 \%$.

\subsubsection{Effect of window size}

The window size used to augment the data is an external parameter apart from other model parameters and it is critical to understand its effect on model performance. It can 
Table 5.6: Random initialization of weights

Pier settlement

\begin{tabular}{ccccc}
\hline \hline Trial \# & ROC-AUC & Accuracy & FNR & F1 score \\
\hline \hline 1 & 0.98 & 0.85 & 0.15 & 0.85 \\
2 & 0.97 & 0.85 & 0.15 & 0.85 \\
3 & 0.98 & 0.86 & 0.14 & 0.86 \\
4 & 0.97 & 0.83 & 0.17 & 0.83 \\
5 & 0.98 & 0.86 & 0.14 & 0.86 \\
\hline$\mu$ & 0.97 & 0.85 & 0.15 & 0.85 \\
$\sigma$ & 0.00 & 0.01 & 0.01 & 0.01 \\
\hline \hline & \multicolumn{2}{c}{ Rupture of tendons } & \\
\hline 1 & 0.92 & 0.69 & 0.31 & 0.69 \\
2 & 0.93 & 0.68 & 0.32 & 0.68 \\
3 & 0.90 & 0.66 & 0.34 & 0.66 \\
4 & 0.91 & 0.65 & 0.35 & 0.65 \\
5 & 0.92 & 0.66 & 0.34 & 0.66 \\
\hline$\mu$ & 0.92 & 0.67 & 0.33 & 0.67 \\
$\sigma$ & 0.01 & 0.02 & 0.02 & 0.02 \\
\hline
\end{tabular}

be observed that the best performance with a combination of maximum ROC-AUC and accuracy and minimum FNR is achieved at 256 samples per window. It is shown in Fig. 5.5 (a), ROC-AUC increases to 1.0 at 512, 800, 1024 samples per window, however, it leads to over fitting with increased FNR. A similar result can be observed from Fig. 5.5 (b) with optimal performance at 256 samples per window. 
(a)

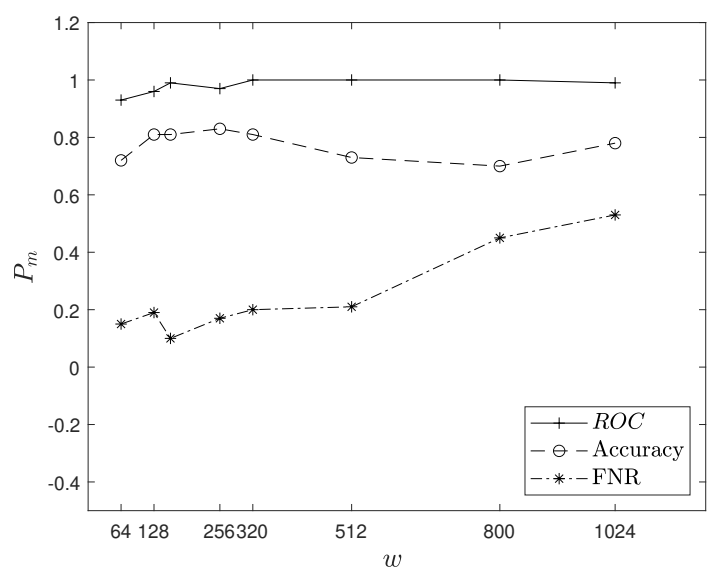

(b)

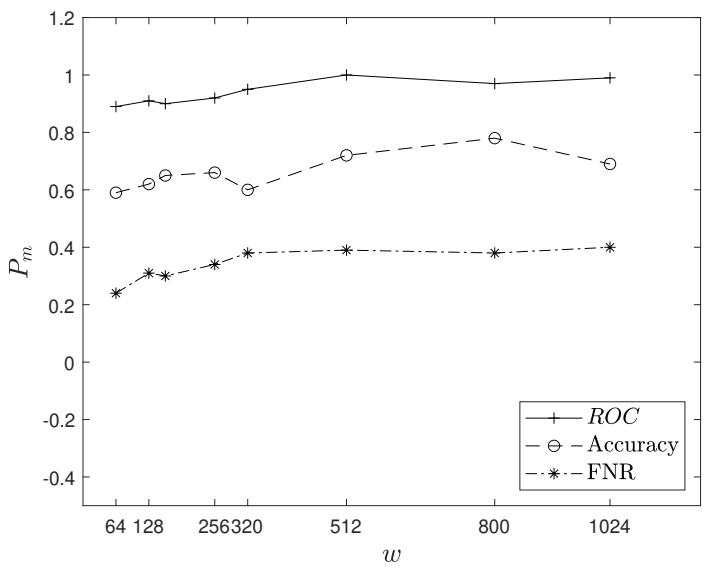

Figure 5.5: Performance evaluation of 1D CNN based on window size for (a) pier settlement and (b) rupture of tendons.

\subsubsection{Model performance}

The optimal parameters are first used to evaluate the performance of the proposed model on an entire series versus voted-windowed samples. The reason for comparison of the entire time-series and voted windows is to show the improved performance by voting the windowed samples. It is observed that voting on windowed dataset increases accuracy considerably and it exhibits in ROC-AUC and precision-recall (PR)-AUC curves, as presented in Fig. 5.6, and 5.7, respectively. It can be observed that voting on windows of non-localized signal increases the probability considerably by allocating the majority class and ignoring the non-prominent class along with augmenting the data samples per class. It may be noted that micro-averaged is the average of area under the curve for all the classes. It may also be noted that the accuracy in case of pier-settlement is 0.83 and it reduced to 0.66 for rupture of tendons as shown in Table 5.7. It can be observed that the FNR increased from 0.17 to 0.34 in case of pier-settlement and rupture of tendons, respectively. The label 0,1 , and 2 are used to denote performance metrics of training, test set of entire time-series and 
windowed time-series, respectively.

Table 5.7: Training and testing performance of 1D CNN.

\begin{tabular}{lccccccccc}
\hline & \multicolumn{7}{c}{ Lowering of pier } \\
\hline \hline Dataset & ROC & PR & A & P & R & FPR & FNR & F1 \\
0 & 0.96 & 0.88 & 0.80 & 0.80 & 0.80 & 0.05 & 0.20 & 0.80 \\
1 & 0.95 & 0.84 & 0.77 & 0.77 & 0.77 & 0.06 & 0.23 & 0.77 \\
2 & 0.97 & 0.91 & 0.83 & 0.83 & 0.83 & 0.04 & 0.17 & 0.83 \\
\hline \hline \multirow{2}{*}{ Dataset } & ROC & PR & A & P & R & FPR & FNR & F1 \\
0 & 0.89 & 0.75 & 0.63 & 0.63 & 0.63 & 0.12 & 0.37 & 0.63 \\
1 & 0.87 & 0.71 & 0.59 & 0.59 & 0.59 & 0.14 & 0.41 & 0.59 \\
2 & 0.92 & 0.82 & 0.66 & 0.66 & 0.66 & 0.11 & 0.34 & 0.66 \\
\hline
\end{tabular}

As shown in Fig. 5.6, voting on samples have improved the AUC for both ROC and precision-recall. It can be observed that in case of pier settlement, there is meager increase on ROC-AUC, however, there is a considerable improvement in the area under the curve for PR. This behaviour can be attributed to a more localized damage in case of pier settlement. As observed in Fig. 5.7, when the damage was considerably distributed in case of rupture of tendons, voting on windows highly increased the PR area under the curve for rupture of tendons. Whereas, ROC-AUC and PR increased by $5.75 \%$ and $15.5 \%$, respectively. 
(a)

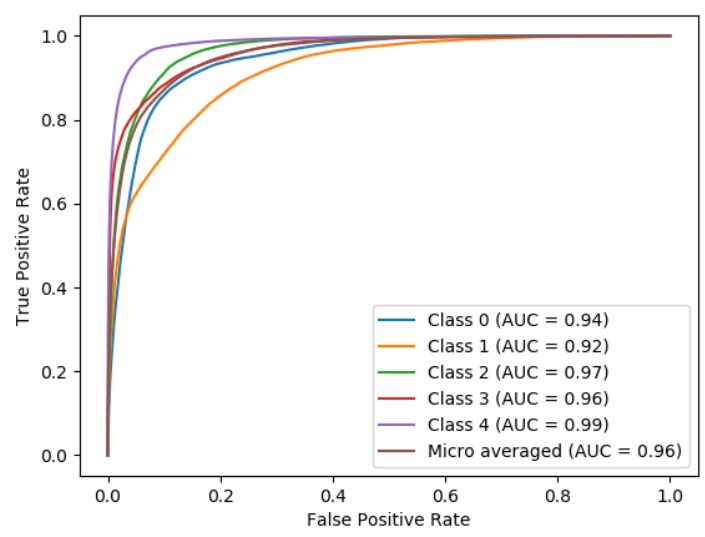

(c)

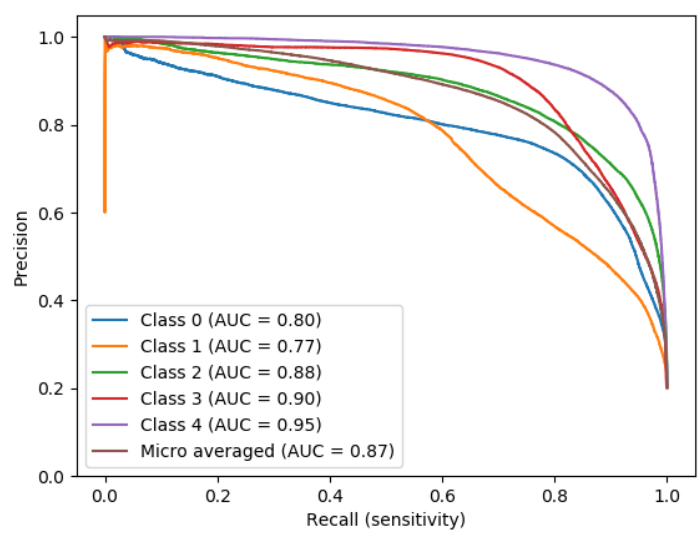

(b)

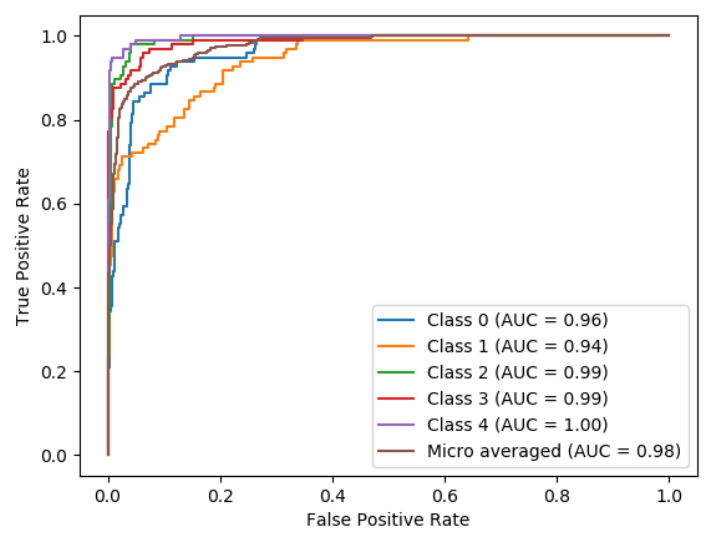

(d)

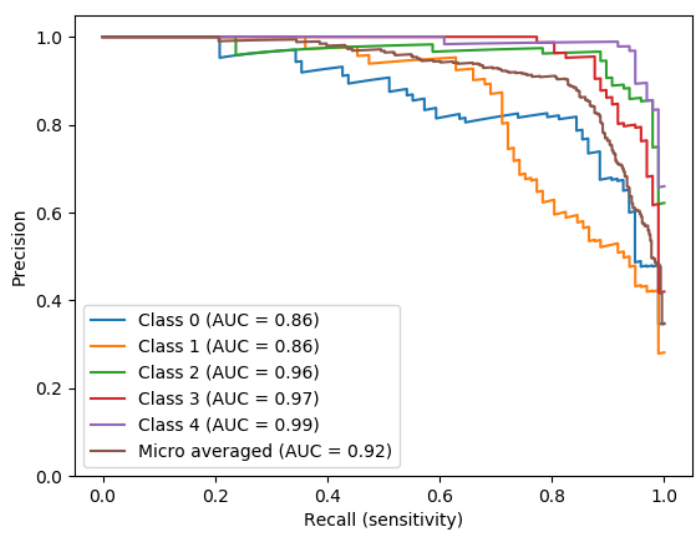

Figure 5.6: Performance of $1 \mathrm{DCNN}$ by windowing of the data of pier settlement (a) seriesROC, (b) windowed-voted ROC, (c) series-PR, (d) windowed-voted PR. 
(a)

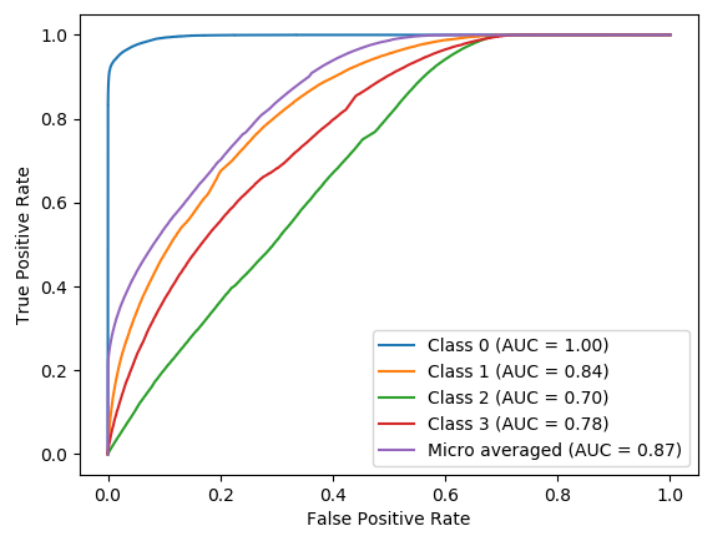

(c)

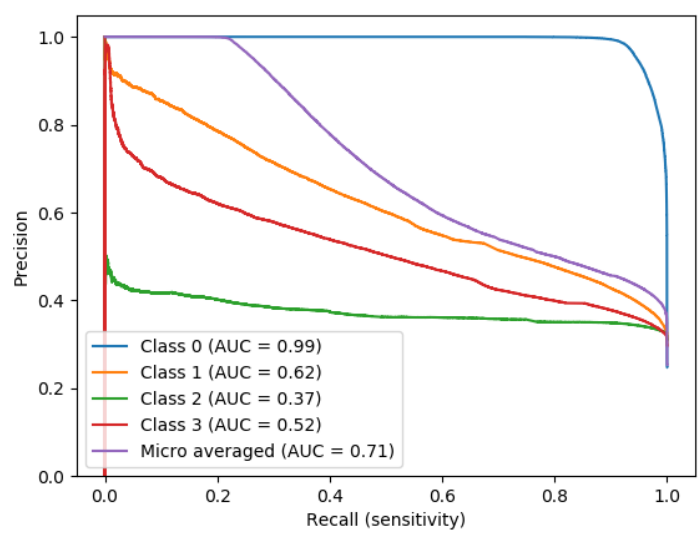

(b)

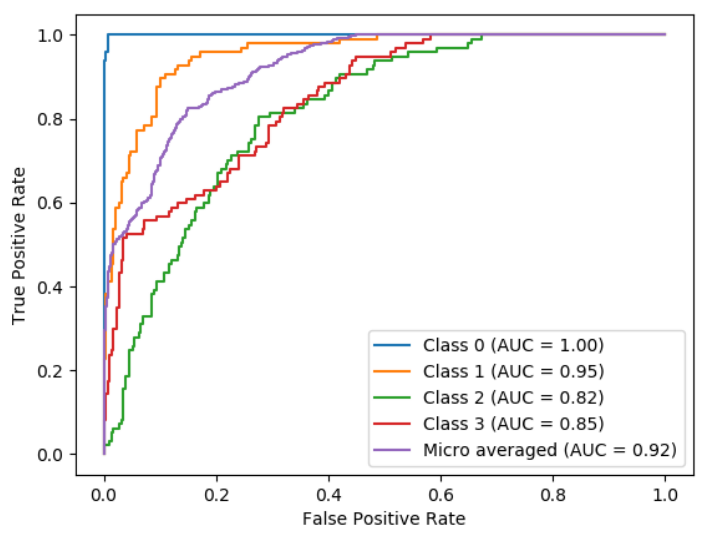

(d)

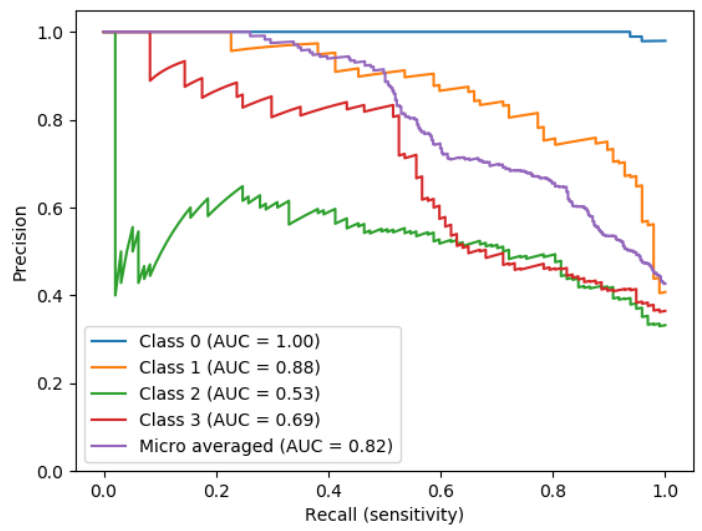

Figure 5.7: Performance of 1DCNN by windowing of the data of rupture of tendons (a) series-ROC, (b) windowed-voted ROC, (c) series-PR, (d) windowed-voted PR.

\subsubsection{Damage localization}

Damage localization is performed using Algorithm 4, for two multiclass damage scenario, namely, pier-settlement and rupture of tendons. The sensor locations are identified first, then, three different structural components of the bridge are used to localize the caused by the pier settlement and rupture of tendons during the demolition period of the bridge. An undamaged pier (Utzenstorf), bridge deck, and damaged pier (koppigen) are used for representation of predicted probability $\left(P_{p}\right)$ and infer damages in three components. The 
Koppigen pier is used for inducing the damage by lowering it in several increments starting with $20 \mathrm{~mm}, 40 \mathrm{~mm}, 80 \mathrm{~mm}$, and moving to $95 \mathrm{~mm}$ at the last stage. Twelve different sensors are used to identify the location of damage, namely, 4 sensors (411, 421, 431, 441) on the undamaged pier (UDP), 4 sensors $(216,221,226,231)$ on the bridge deck (BD), and 4 sensors $(511,521,531,541)$ on the damaged pier (DP), as shown in Fig. 5.8.

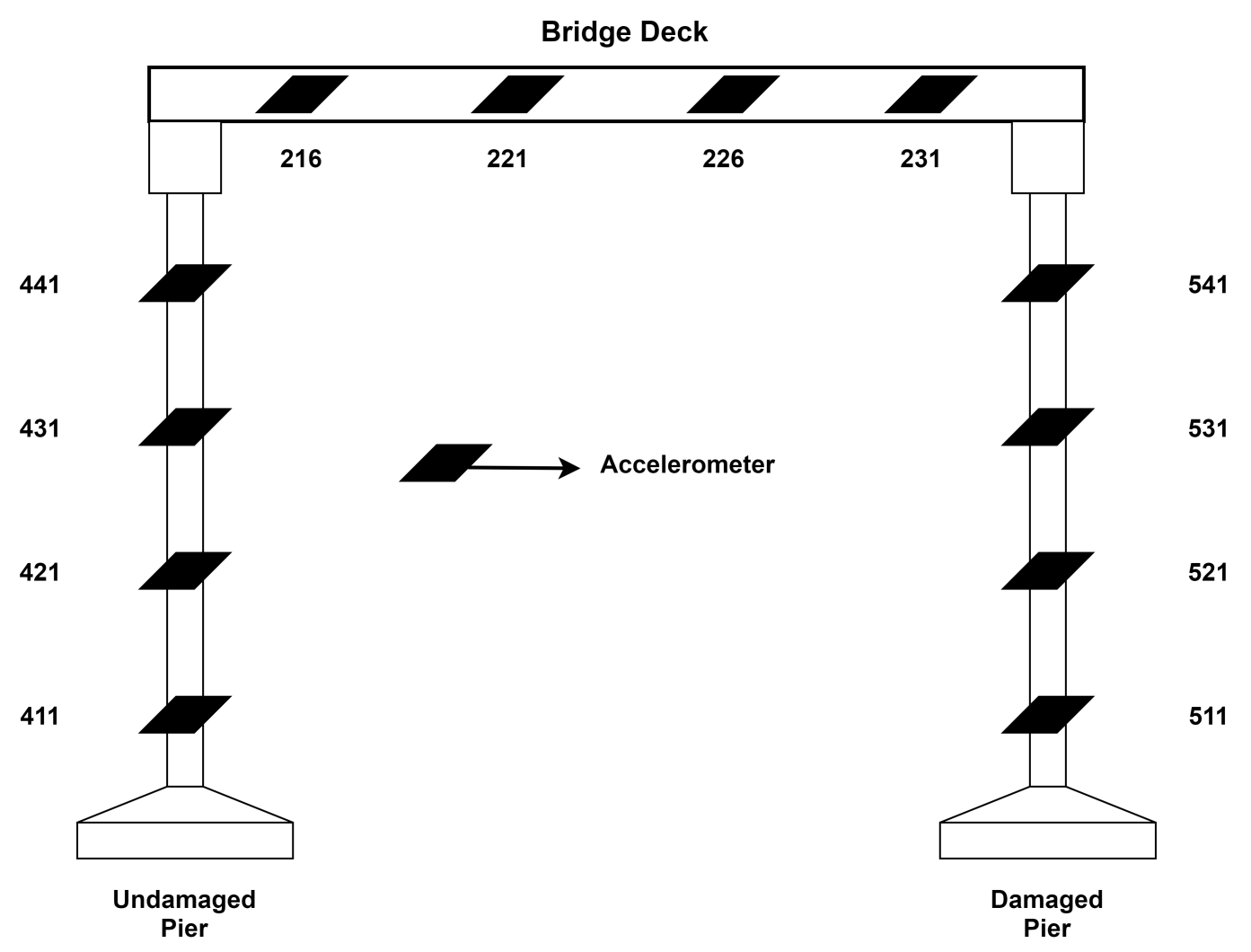

Figure 5.8: Schematic showing the sensor location and their numbers used in the analysis.

The $P_{p}$ is plotted against the sensor number and a dash-dotted average of $P_{p}$ is shown as a representation of combined $P_{p}$ for corresponding structural component, as shown in Fig. 5.9 for $20 \mathrm{~mm}$, and $40 \mathrm{~mm}$ and in Fig. 5.10 for $80 \mathrm{~mm}$, and $95 \mathrm{~mm}$ lowering of pier, respectively. For example, Fig. $5.9(a, b, c)$ represents $P_{p}$ for undamaged pier (UDP), bridge deck (BD), and damaged pier (DP) for $20 \mathrm{~mm}$ lowering of piers. Similarly, Fig. 
$5.9(d, e, f)$ is for $40 \mathrm{~mm}$ lowering of piers, respectively. It can be observed that, unlike in pier settlement of $40 \mathrm{~mm}$, the proposed algorithm does not provide conclusive evidence of nominal damage of $20 \mathrm{~mm}$. However, Fig. $5.10(a, b, c)$ and $(d, e, f)$ shows localization of damage for $80 \mathrm{~mm}$ and $95 \mathrm{~mm}$, and it can be observed that the localization is clearly identified through the proposed threshold where the $P_{p}$ is highest for DP followed by BD which is affected by differential settlement of one of the piers.

$(a)$

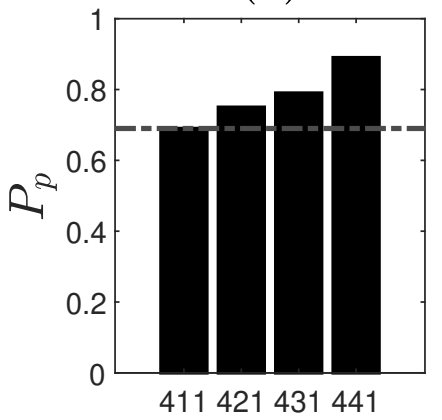

$(d)$

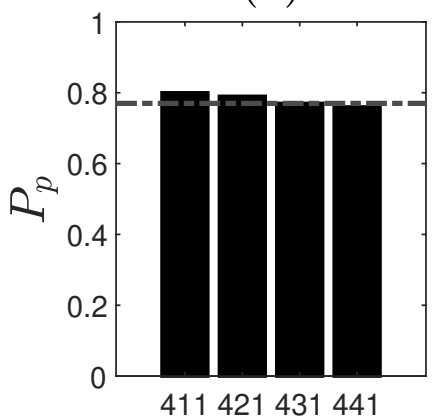

(b)

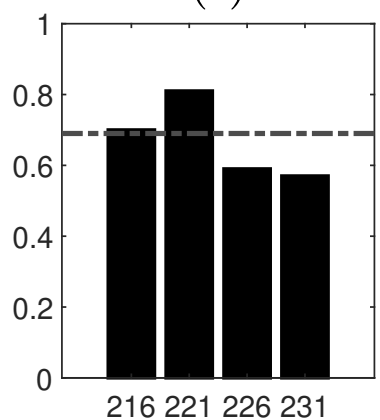

$(e)$

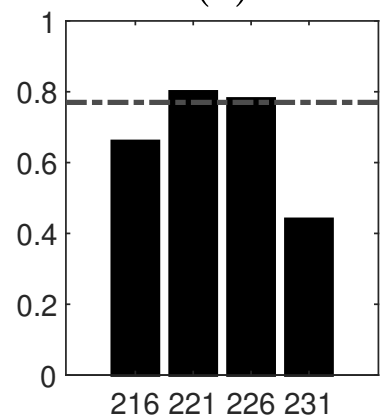

(c)

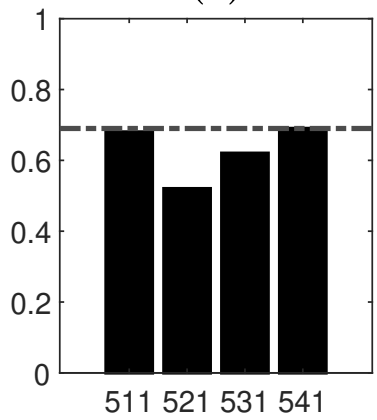

$(f)$

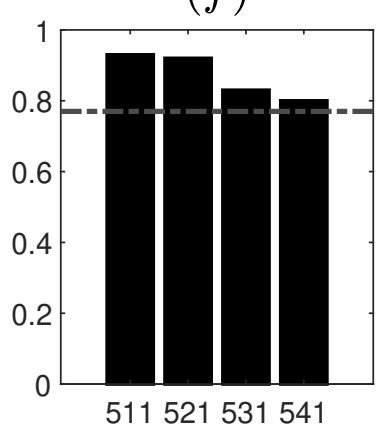

Figure 5.9: Damage localization for pier settlement with two damage levels, $(a, b, c): 20$ $\mathrm{mm}$ and $(d, e, f): 40 \mathrm{~mm}$. 
(a)

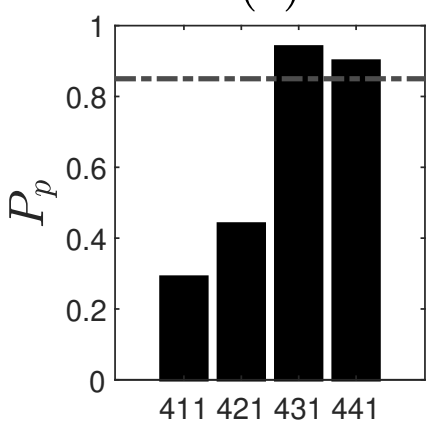

$(d)$

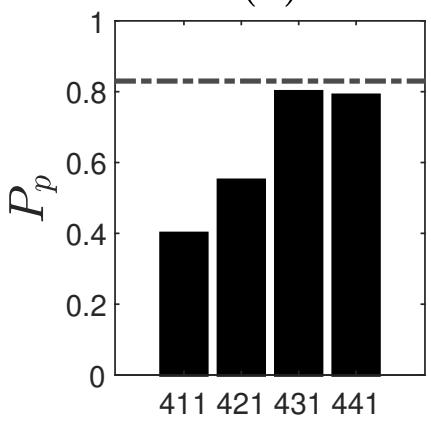

(b)

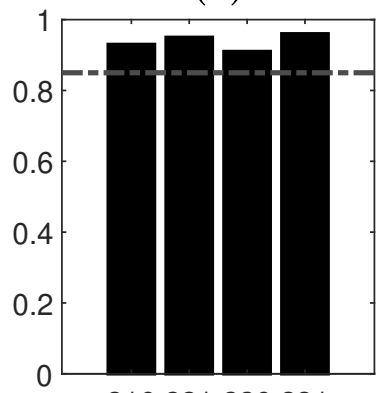

216221226231

$(e)$

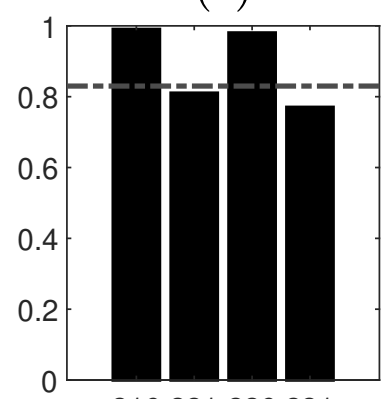

216221226231 (c)

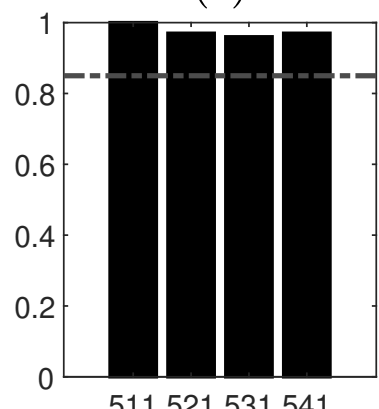

511521531541

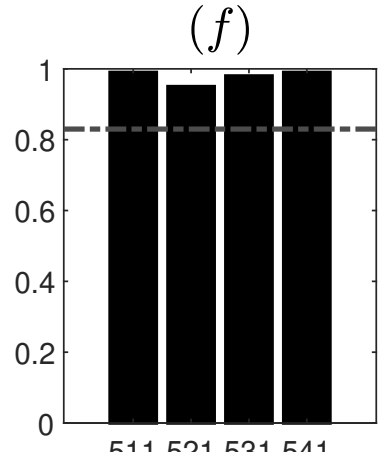

Figure 5.10: Damage localization for pier settlement with two damage levels, $(a, b, c): 80$ $\mathrm{mm}$ and $(d, e, f): 95 \mathrm{~mm}$.

It may be noted that, as the severity increases, the signals become more distinguishable and 1D CNN learns the classification more effectively. It can be observed from Fig. 5.11 that UDP shows lowest predicted probability due to its similarity to the response of the undamaged pier, however, both $\mathrm{BD}$, and $\mathrm{DP}$ shows higher prediction accuracy. The reason for BD's highest probability is attributed to the surface area and larger effect of differential pier settlement of the entire bridge. In summary, it can be concluded that the proposed method performs well for damage levels of $40 \mathrm{~mm}, 85 \mathrm{~mm}$, and $95 \mathrm{~mm}$, however, does not perform well for a very low level of damage, as shown in case of pier settlement of $20 \mathrm{~mm}$. 


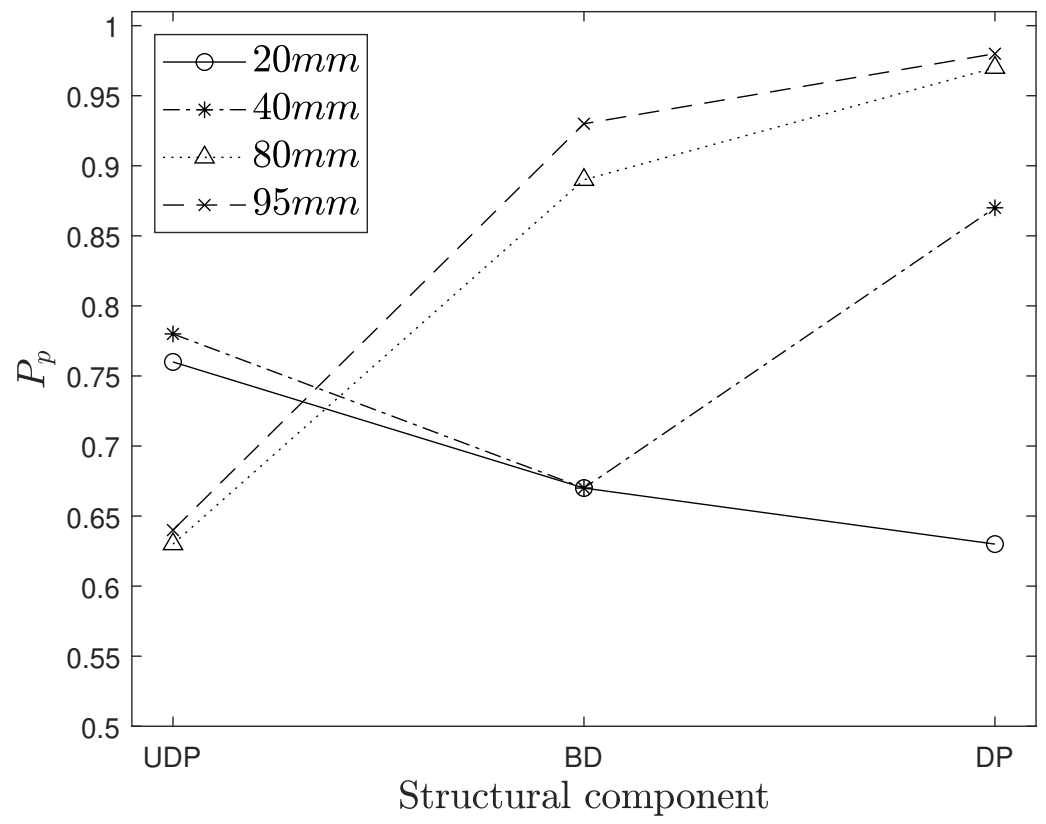

Figure 5.11: Damage localization for the pier settlement.

Similarly, for rupture of tendons, the most affected area would be the bridge deck and the damage induced due to rupture of tendons will create a non-localized and distributed damage throughout the bridge deck in comparison to the bridge piers. The damage localization per sensors is avoided due to non-conclusive inference and a comparison between structural components of the bridge is provided directly in Fig. 5.12. It should be noted that rupture of tendons affects bridge deck highly and it is shown in Fig. 5.12, however, the proposed algorithm could not clearly show the affect of rupture of 2 and 4 tendons, while the rupture of 6 tendons proves to be the worse damage level scenario. 


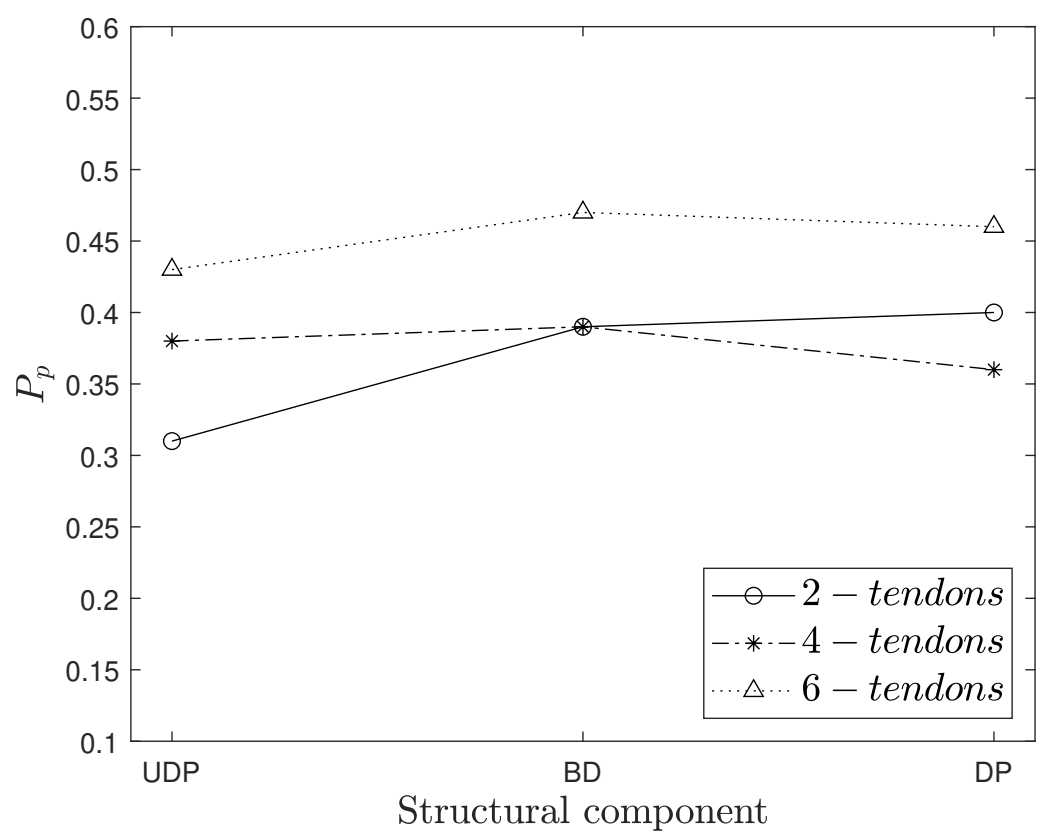

Figure 5.12: Damage localization for the rupture of tendons.

\subsection{Summary}

In this chapter, a windowed-1D CNN is employed for multiclass and multilevel damage detection using limited datasets. Limited dataset is augmented using windowing of the vibration data and the prediction accuracy is improved by a novel voting approach on windowed classes. It is observed that due to non-localization of sensors for data acquisition, damage localization for a minor level of damage (say, $20 \mathrm{~mm}$ of pier settlement) is a challenge to predict. However, the overall accuracy significantly improves with the severity of the damage (i.e., a pier settlement of 40-95 mm). The proposed algorithm is analyzed with a sensitivity analysis on window-size as the external parameter to the model as well as a parametric study to evaluate its sensitivity with random initialization of weights. The improvement in the accuracy is illustrated through a comparison between a single series 
dataset and windowed-voted time-series for ROC and precision-recall AUC. In this work, it is demonstrated that a simple 1D CNN architecture with only one hidden layer is capable of classifying the time-series of vibration data into multi-class with high accuracy. The future work is reserved to improve the algorithm to identify minor level of damage. 


\section{Chapter 6}

\section{Improved Multiclass Damage}

\section{Localization using Windowed Long}

\section{Short-term Memory Networks}

The objective of this chapter is to use limited training data and noisy measurements using deep learning method capable of learning long-term dependencies of the sequential vibration data for continuous and autonomous monitoring of civil infrastructure. In this chapter, a novel Long short-term memory (LSTM) network is employed to improve vibration-based damage detection using limited training data sets and limited sensors. The limited data is augmented by increasing the data per class by splitting the data set using a fixed window approach. The limited sensors are used to identify and localize damage in structural components of the bridge. 


\subsection{Introduction}

Artificial intelligence (AI) methods have successfully been applied to solve challenging tasks in several engineering domains and to automate and improve the classification and data mining tasks [104]. Likewise, AI techniques provide promising opportunities for detection and localization of damages in civil infrastructure by analyzing various sensor measurements with minimal user intervention, thereby reducing cost and increasing accuracy and reliability. SHM community has explored established Machine Learning (ML) algorithms to monitor the condition of infrastructure primarily using structural imagery [111],[120]. Recently, infrastructure monitoring using images of cracks and damage has garnered significant attention as a straightforward autonomous approach to monitor large-scale structures, where Convolutional Neural Networks (CNNs) has gained immense popularity.

Historically, CNN was first introduced to classify low-resolution images of handwritten characters and was named as LeNet [78]. Since then, various CNN models with different architectures were developed. A popular ImageNet CNN model, AlexNet [75], was developed by researchers from the University of Toronto where several layers of convolution and max-pooling were used to train the database. The Visual Geometry Group of Oxford University improved AlexNet and named VGGNet [116] that showed how the depth of CNN influences the accuracy of image reconstruction. The development of new CNN architectures introduced a trend towards using more and more (i.e., deep) layers. Computing giant Google developed a deeper network, GoogleNet [130], with improved dimensionality reduction and computational efficiencies, while ZFNet provided a considerable improvement in classification error rate over AlexNet. 
Primarily designed for object recognition, 2D CNN algorithms were mostly explored for images in various SHM applications to detect defects and anomalies autonomously.[30] presented a vision-based methodology for detecting cracks in concrete structures. The authors used around 40,000 images of damaged and undamaged concrete surfaces collected from various concrete structures to evaluate the accuracy of damage classification using a 2D CNN architecture. [147] proposed a pixel-level CNN to detect cracks on 3D pavement surfaces. The proposed CNN, CrackNet, was made up of two fully connected layers, one convolutional layer, one $1^{*} 1$ convolution layer, and one output fully-connected layer. The proposed network was more efficient than the traditional CNN architecture because of the absence of pooling layers that downsized the output of previous layers. [151] investigated CNN for crack detection in bridges. For bridge damage classification, an AlexNet-based CNN was trained first with around 3800 images of various bridges. For recognition of the bridge components, a ZFNet-based faster regions-CNN was trained with 600 images. To detect cracks, a GoogleNet-based CNN was trained with 60000 cracked and uncracked images. Accuracies of $96.6 \%$ for bridge classification, $90.45 \%$ for bridge component classification, and $99.36 \%$ for crack detection during testing were achieved. Apart from ageing-related damage identification, image-based damage detection also showed significant promises for post-disaster reconnaissance. [81] investigated CNN bridge inspection for system level, component level, and local damage detection. The proposed network was made up of a VGG16 Transfer Learning-based neural network with Bayesian optimization for classification, a faster R-CNN for component detection, and a deep CNN for semantic damage segmentation. Recently, several researchers [143],[16],[129],[119] provided a sys- 
tematic critical review of various deep learning techniques, especially CNN, for structural damage detection. However, it was concluded that acquiring an extensive database of images of the damage in a large-scale structure is still an issue.

Similar to the images of structural damage, SHM researchers have also explored deep learning methods for effective damage detection using temporal information of sequential data, such as, acceleration measurements. For example, [56] proposed sparse coding to extract features from unlabeled acceleration measurements. The damage classification was carried out using CNN, and the results were compared with the traditional machine learning methods, such as, logistic regression and decision trees. A three-span bridge was considered to evaluate the efficacy of the proposed method, and it was shown that sparse coding-CNN based method outperforms other methods with a testing accuracy of $98 \%$. [55] conducted a simulation study on a steel gusset plate connection by varying the size and location of the damage. The measurements were also contaminated with $1 \%$ and $2 \%$ noise to simulate realworld conditions, and CNN was used to classify damage. The proposed method achieved an error of $2 \%$ and showed robustness against environmental noise. Out of various CNN architectures, One dimensional (1D) CNN [74] have shown promising results in capturing the temporal information and in undertaking damage detection using vibration data.

In the recent few years, [4] introduced 1D CNN for a nonparametric vibration-based damage detection. The authors trained the neural network on a vibration signal dataset obtained on a 30-joint steel truss structure, named Qatar Grandstand, by damaging each joint and keeping the other joints undamaged. The proposed model was trained individually on each joint, therefore a total of 31 measurement setups were conducted to develop the 
training database. [99] showed the applicability of 1D CNN with autoencoders for anomaly detection under data compression. The proposed method was validated on a long-span suspension bridge, and an accuracy of $98 \%$ was achieved with a highly compressed dataset and a compression ratio of 0.1. Moreover, [149] extended the applicability of 1D CNN to detect changes in physical parameters of the structures, such as, stiffness and mass. Various structural components such as a beam and steel girder bridges were used for validating the proposed algorithm. Recently. [115] showed the applicability of 1D CNN for damage detection in structural steel frames. Experimental validation was performed on a 2D-steel frame with different damage locations and severity of the damage. The method was shown to identify single as well as multiple damage scenarios. The false-positive rate was evaluated and found to be well within acceptable limits. Furthermore, [84] used transmissibility function-based 1D CNN to effectively identify damage in the ASCE SHM benchmark structure and compared the performance against time-domain and frequency-domain methods. 1D CNN primarily exhibited superior performance over artificial neural networks (ANNs) in the context of computation efficiency and noise imperative for big data.

Apart from the recent progress in 1D CNN, [150] proposed Long Short-Term Memory (LSTM) model, a special class of recurrent neural network, for dam displacement prediction. The authors exploited the long-term dependencies and learning capability of LSTM models to predict the displacement of the dam. The external environmental variables, such as, water pressure, temperature, structural deterioration, and bottom bedrock damage also led to a varied displacement. The study involved optimization of LSTM model to show the effects of the external environment in the resulting displacement. The proposed algorithm 
was compared with various ML algorithms such as support vector machine, multilayer perceptron, multiple linear regression, and boosted regression tree. It was shown that LSTM outperformed the variable selection compared to the other methods. To the best of authors knowledge, the standalone LSTM networks have not been explored in the context of SHM. The main application of a neural network model appears in [4],[3], which presents a 1D CNN for binary damage classification on the Qatar Grandstand dataset. While, the 1D CNN captures relevant information in a neighborhood of samples, it lacks the ability to learn the long-term dependencies of the sequential datasets, which is relevant for structural damage identification over a long period of data for VDD.

In this chapter, a LSTM-based structural damage detection and localization method is proposed. The proposed method captures long-term patterns in an acceleration signal by feeding a sequence of windows extracted from the signal as input to the LSTM model, allowing it to make predictions on the acceleration data. This makes the LSTM architecture a valuable technique in vibration-based SHM. This study makes four novel contributions. First, it introduces a standalone LSTM-based approach for damage localization using acceleration measurements. Second, the limited dataset is augmented by windowing the acceleration measurements and a novel approach of voting on the prediction class for windowed-dataset is presented to increase the prediction accuracy. Third, a thorough hyperparameter tuning analysis was conducted followed by the random initialization of the weights for tuned parameters, comparing the results with 1D CNN. Fourth, the proposed method is demonstrated for multiclass and multilevel damage identification in a full-scale bridge. To the authors' best knowledge, it is the first time that LSTM-based 
model has been presented for multi-class damage identification.

The chapter is structured as follows. A brief introduction of the structural damage identification using deep learning techniques is presented in Section 6.1, followed by the gap areas of the existing research and novelty of the proposed method. Next, Section 6.2 presents the proposed methodology based on LSTM networks along with the data pipeline and performance metrics to identify and localize damage. The results including both binary and multiclass damage localization are illustrated in Section 6.3. This section also highlights the importance of hyperparameter tuning and evaluation of optimal window size along with the sensitivity to random initialization of weights. The key conclusions of the proposed research are described in Section 6.4 .

\subsection{Proposed Methodology}

In this chapter, a novel method for damage classification and localization is proposed using an LSTM network. In this section, first, a theoretical explanation of the proposed method is provided and next, the performance metrics for evaluating the proposed method are discussed.

\subsubsection{Preliminaries of LSTM model}

In this section, a method based on a Long Short-Term Memory (LSTM) network [62] is proposed to classify the vibration measurement into damage levels. Given an input acceleration signal, $x=\left(x_{1}, \ldots ., x_{T}\right)$, a standard recurrent neural network computes

hidden vector sequence as $h=\left(h_{1}, \ldots \ldots, h_{T}\right)$ and the output vector sequence as $y=\left(y_{1}\right.$, 
$\left.\ldots \ldots, y_{T}\right)$ by iterating Eqs. $(6.1-6.2)$ from time, $t=1$ to $T$ :

$$
h_{t}=\mathcal{H}\left(W_{i h} x_{t}+W_{h h} h_{t-1}+b_{h}\right)
$$

where $W$ denotes weight matrices, $W_{i h}$ is the input-hidden weight matrix, $W_{h h}$ is the hidden-hidden weight matrix, $b$ denotes bias vectors, $b_{h}$ is hidden bias vector, and $\mathcal{H}$ is the hidden layer activation function. The output can be found as,

$$
y_{t}=W_{h o} h_{t}+b_{0}
$$

where $y_{t}$ denotes output at time $t, W_{h o}$ is the hidden-output weight matrix, and $b_{o}$ is output bias vector. $\mathcal{H}$ is usually an element-wise application of a sigmoid function. Fig. 6.1 illustrates a typical single LSTM memory cell [33]. The transformations applied to the input $x_{t}$ and hidden state from the previous time step $h_{t-1}$ in the LSTM cell are described by Eqs. (6.3-6.8). 


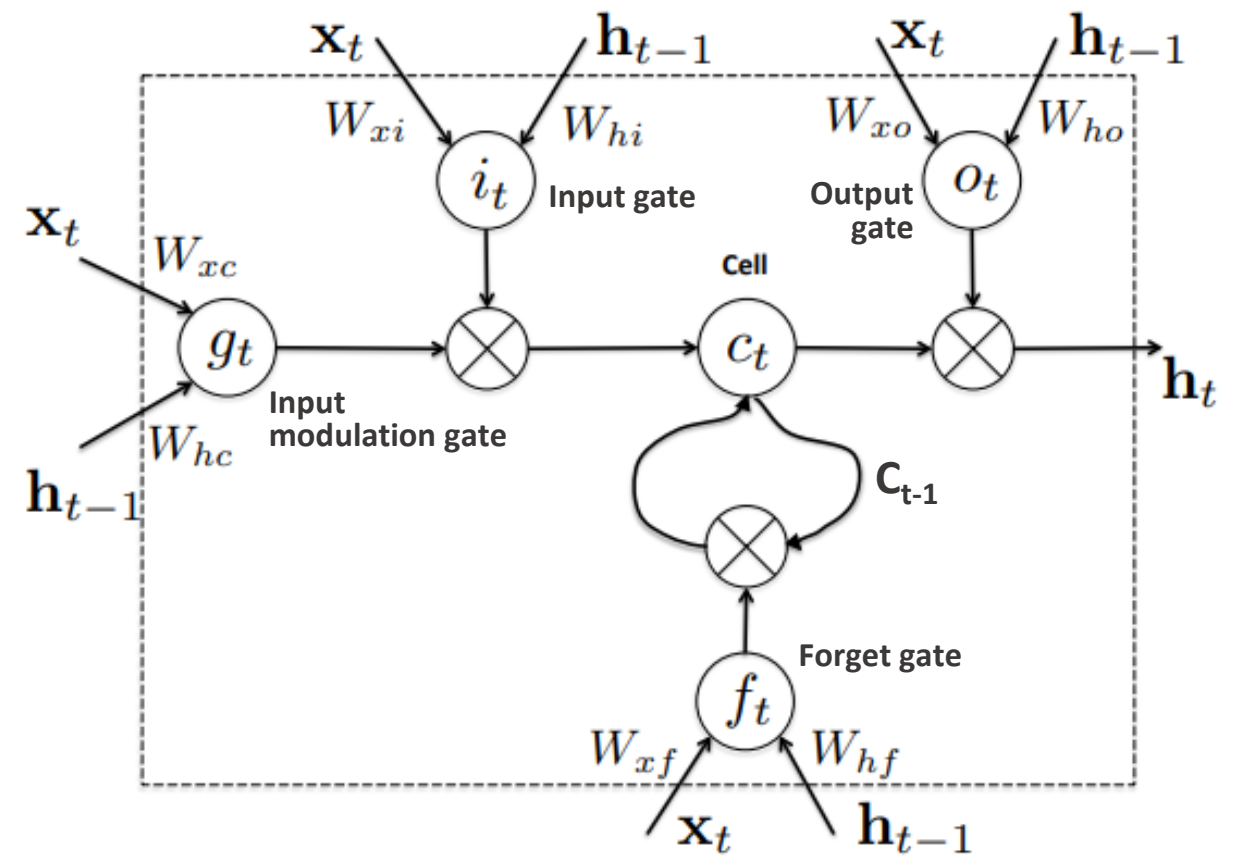

Figure 6.1: The typical internal structure of an LSTM cell [33].

$i_{t}=\tanh \left(W_{x i} x_{t}+W_{h i} h_{t-1}+b_{i}\right)$

$$
f_{t}=\sigma\left(W_{x f} x_{t}+W_{h f} h_{t-1}+b_{f}\right)
$$

$$
g_{t}=\tanh \left(W_{x c} x_{t}+W_{h c} h_{t-1}+b_{c}\right)
$$

$$
o_{t}=\sigma\left(W_{x o} x_{t}+W_{h o} h_{t-1}+b_{o}\right)
$$

$$
c_{t}=f_{t} \otimes c_{t-1}+i_{t} \otimes g_{t}
$$




$$
h_{t}=\tanh \left(c_{t}\right) \otimes o_{t}
$$

where $\sigma$ is the logistic sigmoid function, and $i, f, g, o$ and $c$ are, respectively, the input gate, forget gate, input modulation gate, output gate and cell activation vectors, all of which are the same size as the hidden vector $h$. The weight matrix subscripts have the same meaning, for example, $W_{h i}$ is the hidden-input gate matrix, $W_{x o}$ is the input-output gate matrix. The bias terms (which are added to $i, f, g$, and $o$ ) have been omitted in Fig. 6.1 for clarity. The weight matrices represent the learnable parameters of the model, while gradient decent algorithm is used to minimize prediction error on a training set.

The cell state $c_{t}$ encodes the information of the sequence observed up to that time step. The input gate controls the information added to the cell state from the current time step, and the forget gate controls what information needs to be forgotten from the current cell state. For example, if the output vector of the forget gate $f_{t}$ has a near-zero value in the first dimension, it indicates that the first dimension of the cell state $c_{t}$ needs to be "forgotten". The forgetting occurs in the element-wise multiplication, i.e., multiplying an element by a near-zero value results in a near-zero element in the output vector. By maintaining cell state in this manner, the LSTM cell is able to capture both long and short term relationships between the input time-series values and the predicted variable (e.g., damage classification). During training, the truncated back propagation is used on truncated sequences to make the process computationally feasible. During prediction, the forward pass can be applied to arbitrarily long sequences (the LSTM cell can be repeatedly applied to any number of input time steps). More details of the internal structure and 
training of LSTM can be found in (Chen 2016).

\subsubsection{Damage detection using the proposed LSTM model}

The proposed deep learning model for the time-series level classification is a multi-layer LSTM network architecture, as shown in Fig. 6.2. The pre-processed sequences of windows are given as input to the model, and the output of the final LSTM time step is considered as the prediction of a sequence (the set of classification probabilities $P\left(y=c_{t}\right)$ to each class $c_{t}$ ). During training, forward and backward passes are performed on the input sequences, and the weight updates are made to minimize the cross-entropy loss on a batch of sequences. The predicted set of classification probabilities $P_{p}\left(y_{c}\right)$ for a full acceleration measurement is obtained by summing the class probabilities of all the window sequences in a single signal series. The class with the maximum probability is the predicted damage level classification of the series. It may be noted that this is equivalent to voting on the classification probabilities of individual window sequences to arrive at the prediction of the

full series. It is observed that the voting process improves the prediction accuracy and other evaluation metrics on the time-series test data. 


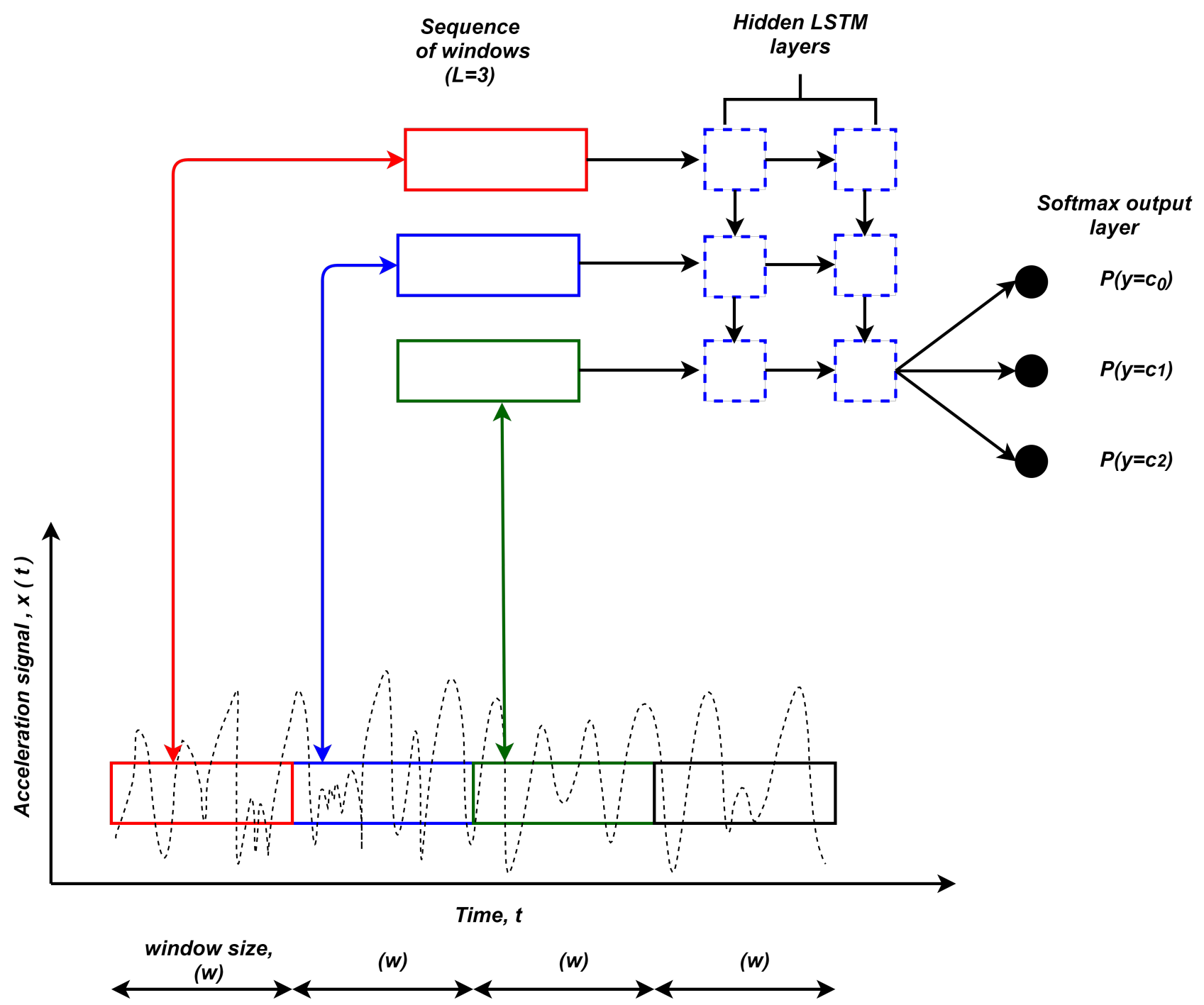

Figure 6.2: Extracted sequences of windows from the vibration signals in the LSTM network.

In the proposed method, the window size $w$ and the sequence length $L$ (number of windows in a sequence) become hyperparameters that are tuned to improve the accuracy of the neural network. The hyperparameters of LSTM network include a number of layers and a finite number of nodes in each layer (network architecture), activation function, and batch size responsible for performing weight updates during the training. Optimal parameters are found using a random search on a hyperparameter space [22]. In each iteration, the 
search algorithm randomly selects a configuration of values for hyperparameters from a specified set of possible values, and trains three models with those parameters on three splits of training and validation sets, resulting in a 3-fold validation. The hyperparameters configuration that gives the highest mean validation accuracy is selected as the final tuned set of hyperparameters of the model. A training session is terminated when, either a specified maximum number of epochs is reached, or the validation loss does not decrease for a specified number of epochs (i.e., early stopping). The final network weights are taken from the epoch with the smallest validation loss.

The purpose of the proposed method is to classify acceleration responses depending on various damage levels and classes. The classification problem is presented as binary (undamaged vs. damaged) or multiclass (undamaged, and damage of more than two levels). Fig. 6.3 illustrates the proposed data pipeline, which consists of a series of pre-processing and post-processing steps with an LSTM network as the classification model. It should be noted that the solid black lines represent datasets, blue dotted lines represent operation on the datasets, and multiple arrows represent multiple data instances. A single acceleration time-series consists of a large number of samples (for example, a record of long vibration data). In the proposed method, the acceleration is first normalized with respect to its mean and standard deviation. This improves the convergence rate of models trained on the datasets and prevents outliers from dominating the input [67]. Second, the segment of the scaled time-series is fed into a sequence of continuous windows (window size $w$ ), and a sequence of such windows (length $L$ ) is arranged to form one input instance to the LSTM network. Thus, the input of the network is a $w$-dimensional sequence of length $L$. Many 
such sequences can be extracted from a single original acceleration time-series, and each sequence is assigned to a label (damage level) of the original series.

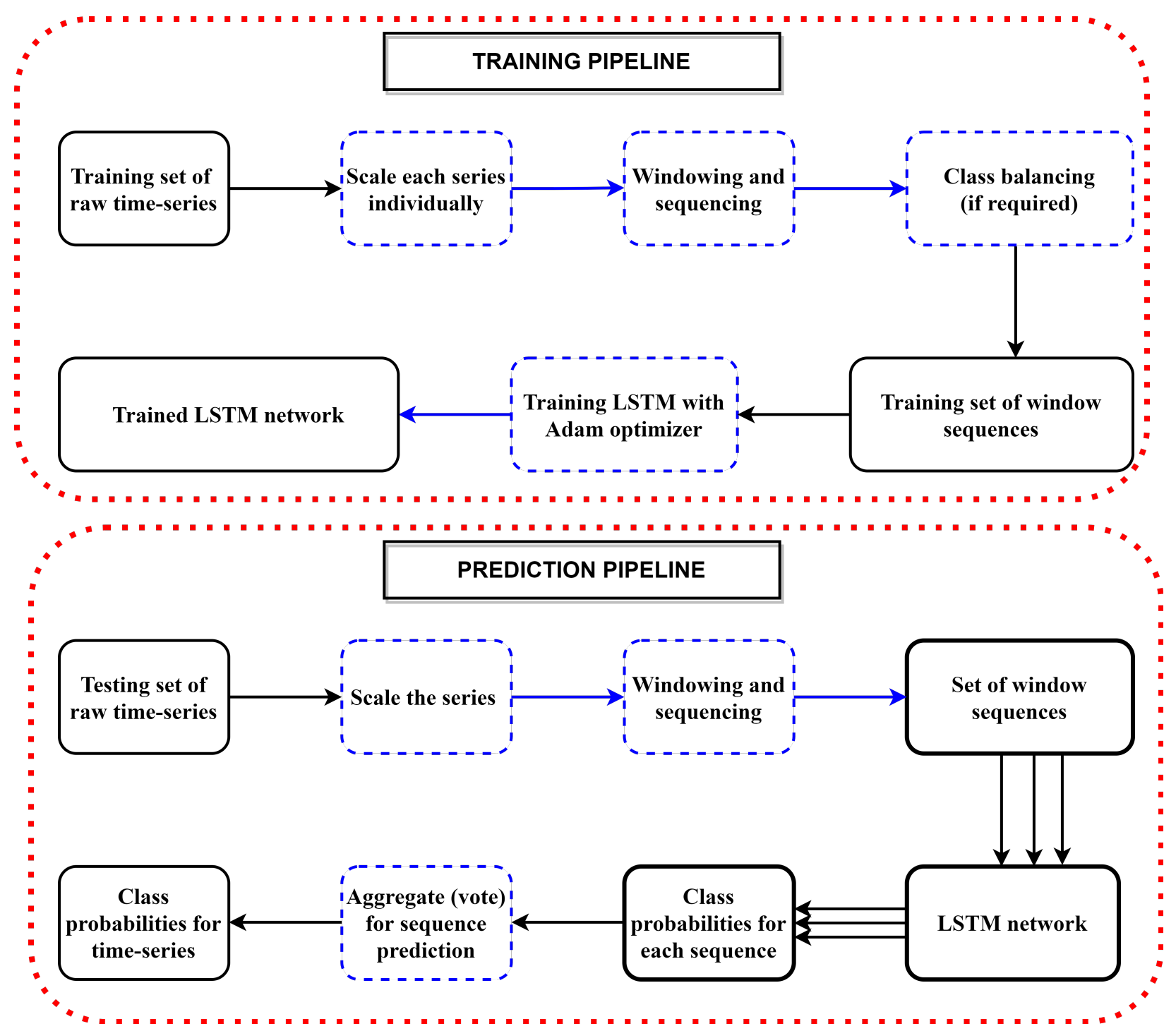

Figure 6.3: Data pipelines for training and prediction of the LSTM network from the acceleration response.

The process of extracting sequences of windows from a time-series is illustrated in Fig.

6.2. This technique of transforming the original series into sequences of windows effectively reduces the data dimension, and additionally, it increases the training set size (multiple sequences per time-series), which in turn allows training deep learning models with less 
over-fitting. Imbalanced data can cause problems in model training. To alleviate this problem, a balanced dataset is created by preparing training set by randomly selecting a number of undamaged sequences equal to the number of damaged sequences.

\subsubsection{Damage localization using the proposed LSTM model}

The LSTM network described in the previous section produces a set of probabilities $P_{p}\left(y_{c}\right)$ for a given acceleration signal. These indicate the probability that the signal belongs to each class $c$. Multiple signals are obtained from accelerometers placed in the critical locations of a structure, and model prediction probabilities are computed for each signal. In this manner, a probability of damage distribution over the space of the structure can be estimated, and locations with high probability of damage can be identified. This damage probability distribution over the structure can be visualized (for example, heatmaps of probability values over a 2D structure) to aid an engineer in quickly localizing damages. Algorithm 5 summarizes the proposed approach for damage detection and localization.

\subsubsection{Performance criteria}

Several metrics are used to evaluate the performance of a classification model. These metrics measure different aspects of the obtained results. A brief description of the selected metrics is provided below and explained in the context of SHM of civil infrastructure. The primary form of prediction results is given by the confusion matrix, which is a tabulation of classifications made by a model. It shows the "classification distribution" of a model, and helps identify properties of the model, such as when it is consistently misclassifying 


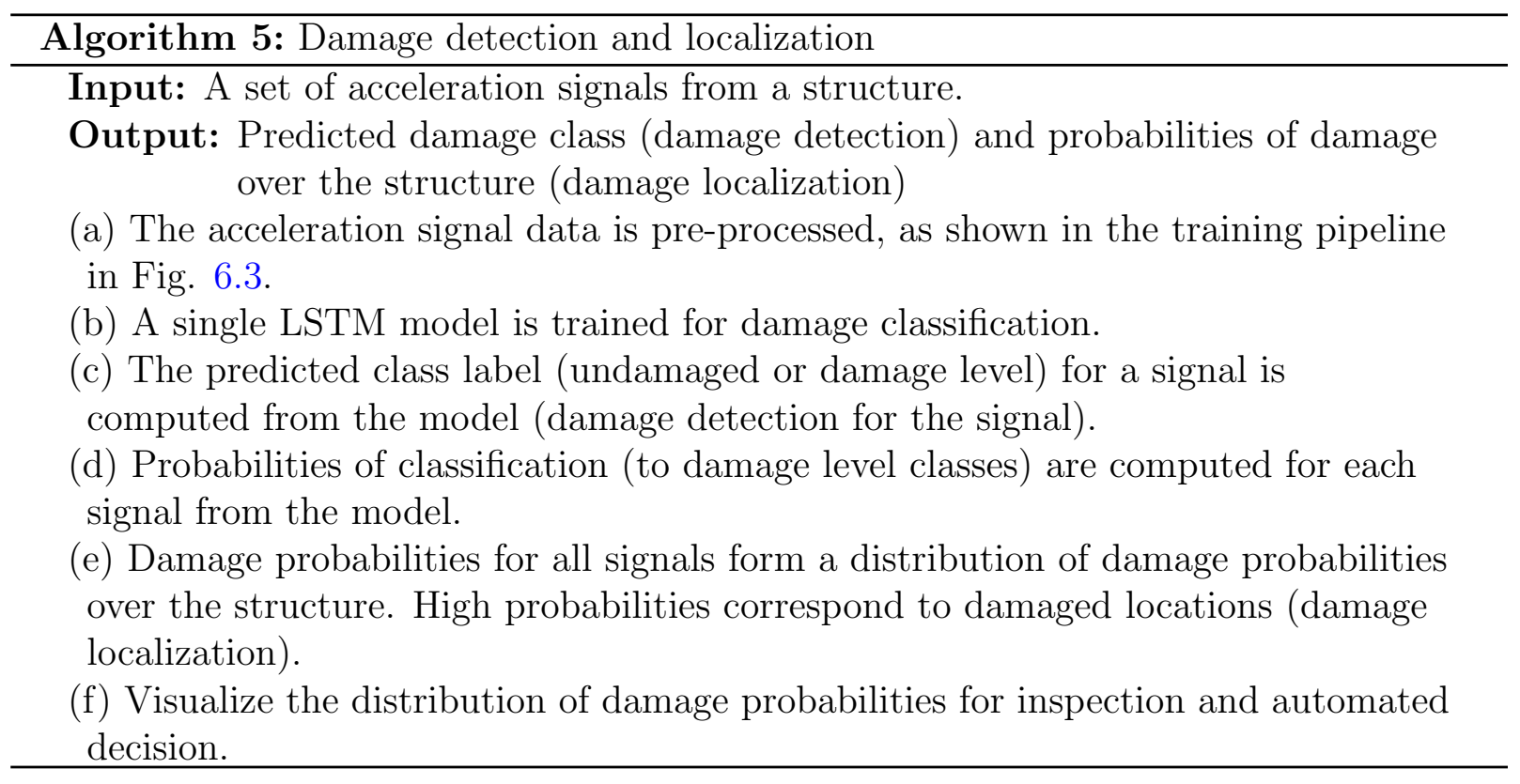

one class as another. The confusion matrix is obtained for both binary and multiclass classifications. Table 6.1 shows the confusion matrix for the case of binary classification. It should be noted that True Positives is denoted as TP, True Negatives as TN, False Positives as FP, and False Negatives as FN. Multiple metrics can be derived from the confusion matrix, as shown in Table 6.2.

Table 6.1: Confusion matrix for a binary classification problem.

\begin{tabular}{cc|cc} 
& & \multicolumn{2}{|c}{ Predicted class } \\
\hline \multirow{3}{*}{ Output class } & Damage & TP & FN \\
& Healthy & FP & TN \\
\hline
\end{tabular}

In this study, two key metrics are used as the performance metrics to evaluate the proposed method, namely, accuracy, and FNR. Accuracy is the primary evaluation metric to understand the ability of the model to correctly classify the inputs. A false negative (type II error) represents a truly damaged series that is classified as undamaged by the model, 
Table 6.2: Description of the selected performance metrics.

\begin{tabular}{|c|c|c|}
\hline Metric & Formula & Remarks \\
\hline \hline ROC-AUC & Recall Vs FPR & Degree of separability between classes \\
\hline Accuracy & $\frac{\mathrm{TP}+\mathrm{TN}}{\mathrm{TP}+\mathrm{FN}+\mathrm{FP}+\mathrm{TN}}$ & Less useful for heavily imbalanced data \\
\hline Precision & $\frac{\mathrm{TP}}{\mathrm{TP}+\mathrm{FP}}$ & Positive predicted value \\
\hline Recall & $\frac{\mathrm{TP}}{\mathrm{TP}+\mathrm{FN}}$ & True positive rate or sensitivity \\
\hline False Positive Rate $(\mathrm{FPR})$ & $\frac{\mathrm{FP}}{\mathrm{TN}+\mathrm{FP}}$ & False alarm when there is no damage \\
\hline False Negative Rate $(\mathrm{FNR})$ & $\frac{\mathrm{FN}}{\mathrm{TP}+\mathrm{FN}}$ & No alarm for actual damage \\
\hline
\end{tabular}

which could lead to catastrophic consequences in a critical structure. Therefore, the FNR is measured and compared in the model evaluation experiments in this work. Two plots highlight the trade-off between metrics as the decision threshold of the classifier changes: the Receiver Operating Characteristic (ROC) curve shows the trade-off between FPR and true positive rate, and the precision-recall (PR) curve shows the trade-off between precision and recall. By analyzing these curves obtained from a test set of model predictions, an engineer can make an informed decision on the balance that is needed between the metrics and make a decision on threshold suitable for the task. The PR curve, in particular, is suitable for the evaluating the cases where the datasets are highly imbalanced. In this study, ROC-AUC and PR-AUC curves are plotted for visual comparison. The corresponding area-under-the-curve (AUC) of these graphs (ROC-AUC and PR-AUC) represent an aggregate measure of the model's ability in terms of the relevant metrics. The damage localization results are evaluated by inspecting the visualization of damage probabilities 
over the structure, and verifying that high damage probabilities have been assigned to the damaged location, while the other locations are assigned with low damage probabilities.

\subsection{Performance Evaluation}

The proposed method is evaluated in two studies: an experimental setup, and a full-scale bridge. For performance evaluation, accuracy, FNR, ROC-AUC and PR-AUC are used. Several parametric studies are conducted to evaluate the performance of the proposed method with the window size in the model input, and the effect of voting on individual windows to obtain the final prediction.

\subsubsection{Experimental study}

Damage detection where the classification is undertaken between one of two classes, such as damaged and undamaged, is called a binary classification. In this section, the experimental benchmark data of Qatar University Grandstand Simulator (QUGS) [4] is used to evaluate the performance of the proposed method. The QUGS was constructed to evaluate and develop effective structural damage detection techniques suitable for monitoring of modern stadia, as shown in Fig. 6.4. The frame was designed to carry a total of 30 spectators with area dimensions of $4.2 \mathrm{~m} \times 4.2 \mathrm{~m}$. The design considerations used for the experimental test structure was to guarantee its safety and compatibility with the specifications of modern grandstands. The structure was utilized in its current form (steel frame only) to generate vibration data under several structural damage cases. The grandstand was excited using a shaker and two 16-channel data acquisition systems were used to acquire the acceleration 
responses. A total of 31 scenarios are considered: the first scenario corresponds to the reference (undamaged) case, while in scenarios 2 to 31, damage was introduced by loosening the bolts at the joints 1 to 30, respectively, as shown in Fig. 6.5. A total of 30 accelerometers were mounted at the joint of each girder and the filler beam, and a magnetic mounting plate was used to attach the sensors on the frame. The acceleration data was sampled at $1024 \mathrm{~Hz}$ and collected for 256 seconds under a band-limited white noise excitation from the shaker with a frequency range of $0-512 \mathrm{~Hz}$. A range of values for hyperparameters is used for tuning the LSTM model using a random search, as shown in Table 6.3. The window size is varied as an external parameter between 64 and 512 samples. Various values for other hyperparameters, such as, the number of hidden layers (range of 1-6), and nodes in hidden layers (range of 32-1024) are considered to achieve optimal performance of the proposed model. Consequently, the random search algorithm explores shallow, wide and deep LSTM architectures. The optimal hyperparameter configuration obtained using random search algorithm for QUGS experiment is presented in Table 6.4. It can be observed that the highest performing window is 64 , and it requires at least three hidden layers for best accuracy. A comparison is drawn between the window-size $(w)$ and the performance metric $\left(P_{m}\right)$ to understand model performance with changing $w$. Three different metrics are used, namely, ROC-AUC, accuracy, and FNR as these three metrics broadly cover the efficacy and any shortcomings of the classification model. The result is shown in Fig. 6.6. 


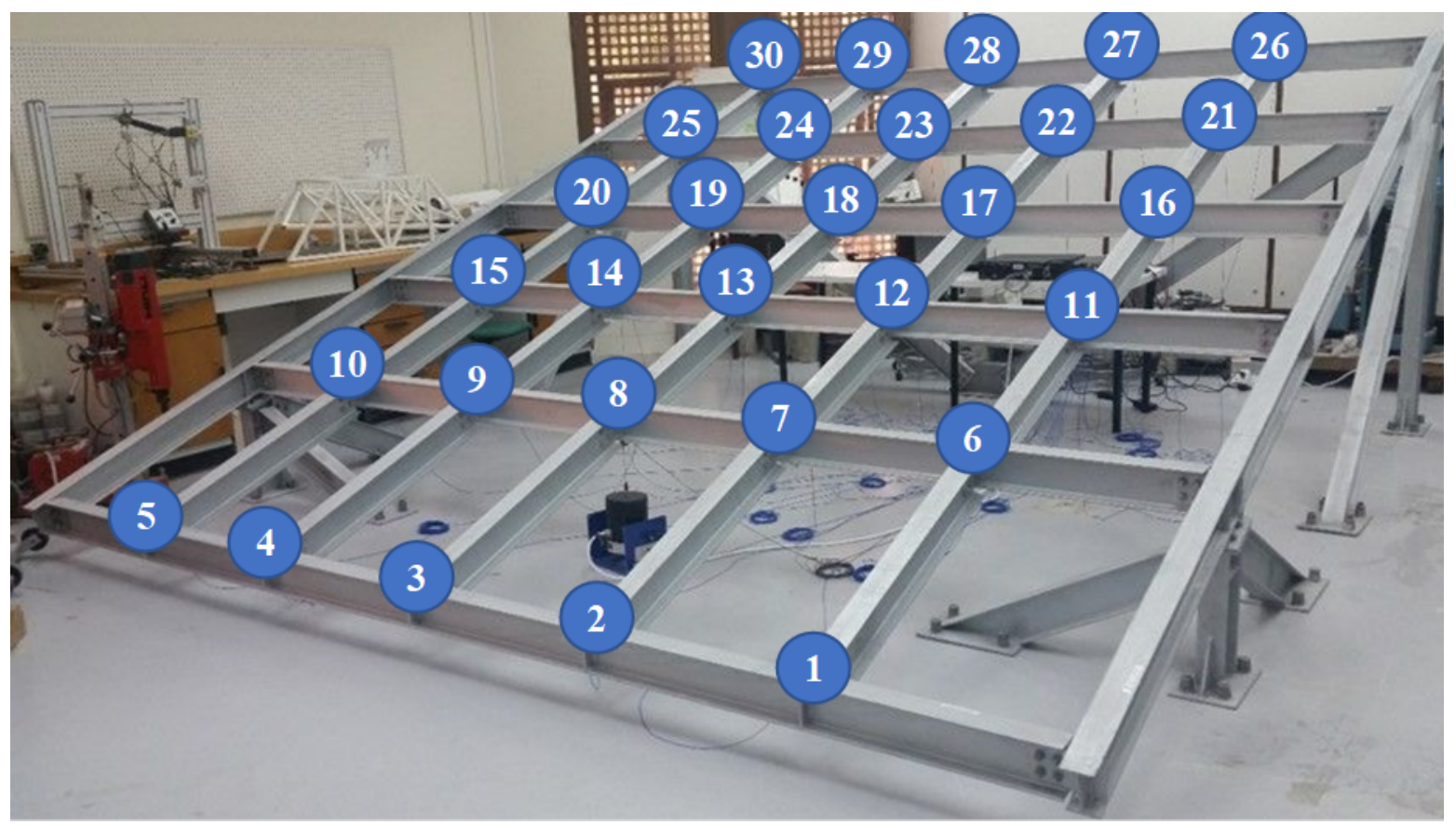

Figure 6.4: QUGS testbed, where the joints are numbered from 1 to 30 [4].

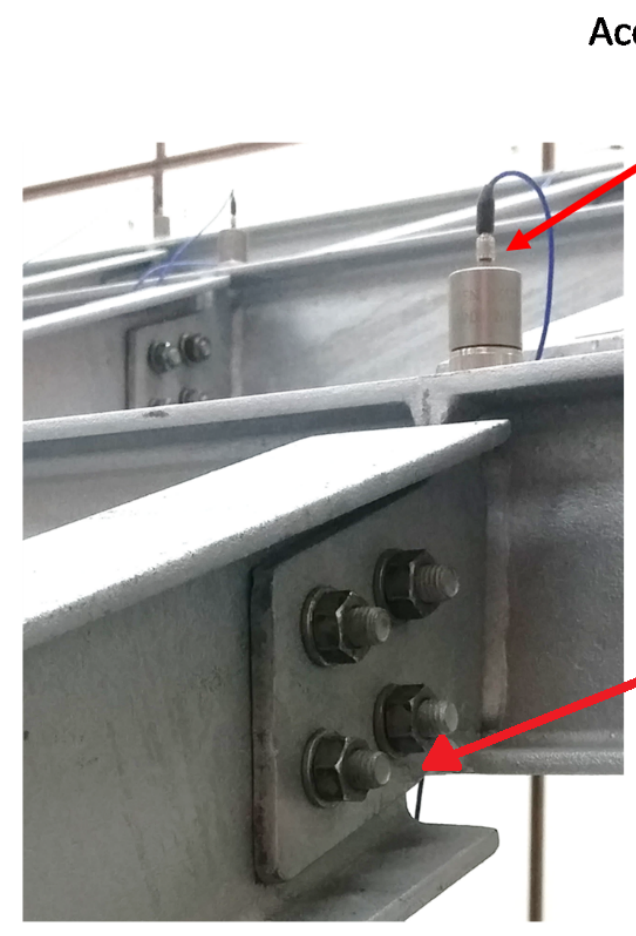

(a)

\section{Accelerometer at the Joint}

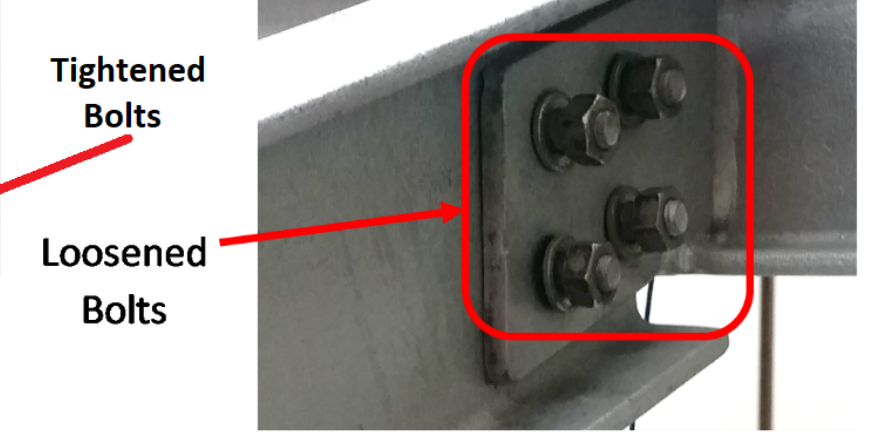

(b)

Figure 6.5: Undamaged and damaged state of the girder and beam joint in the QUGS [4]. 
It can be observed that $w=64$ and $w=128$ yield the best performance metric values (high accuracy and ROC-AUC with low FNR), and performance metrics consistently degrade with increasing window size. This behavior is attributed to a reduction in the data samples (sequences of windows) with increasing $w$, causing the LSTM network to overfit when trained on smaller datasets.

Table 6.3: Hyperparameters used for tuning LSTM by random search algorithm.

\begin{tabular}{ll}
\hline Parameter & Values \\
\hline \hline Window size & $64,128,160,256,512$ \\
No. of windows in a sequence & $2,4,8,16$ \\
No. of hidden layers & $1-6$ \\
No. of hidden nodes & $1024,512,256,128,64,32$ \\
Dropout rates & 0.2 and 0.5 for each hidden layer \\
Learning rate & $0.0003,0.001,0.01$ \\
Batch size & $64,256,512$ \\
Cell type & LSTM with tanh activation \\
\hline
\end{tabular}

Table 6.4: Optimal configuration of the LSTM hyperparameters for QUGS data.

\begin{tabular}{ll}
\hline Parameter & Values \\
\hline \hline Window size & 64 \\
No. of windows in a sequence & 8 \\
No. of hidden layers & 3 \\
Architecture & {$[64,128,64,32,1]$} \\
Dropout rates & 0.2 \\
Learning rate & 0.001 \\
Batch size & 256 \\
Training epochs & 100 with early stopping \\
\hline
\end{tabular}




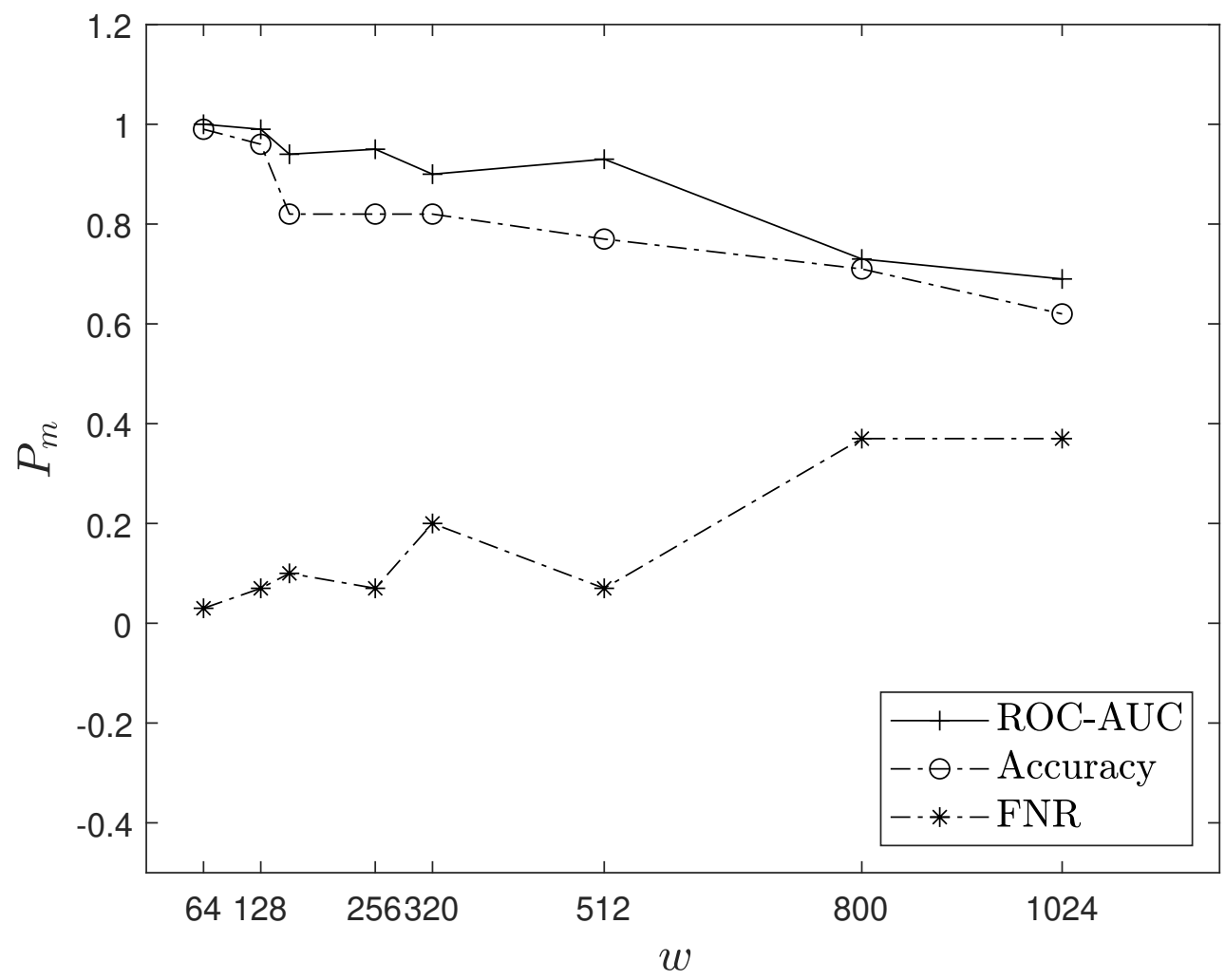

Figure 6.6: Performance evaluation of the proposed LSTM model based on window size in the QUGS data.

To compare the proposed LSTM approach with the 1D CNN method introduced by Abdeljaber et al. (2017), a 1D CNN is trained with the same hyperparameter tuning process as outlined before. The optimal 1D CNN network has 4 hidden layers with nodes $256,128,64,32$ in each layer and a kernel size of 64 . After acquiring the optimal tuned parameters, a variability study is conducted to understand variance in the metrics by training the LSTM and 1D CNN models with random initialization of network weights of 5 times. The result is shown in Fig. 6.7. It was found that both models perform well consistently with accuracy at 1.0 and FNR at 0.0 with negligible variability. 
(a)

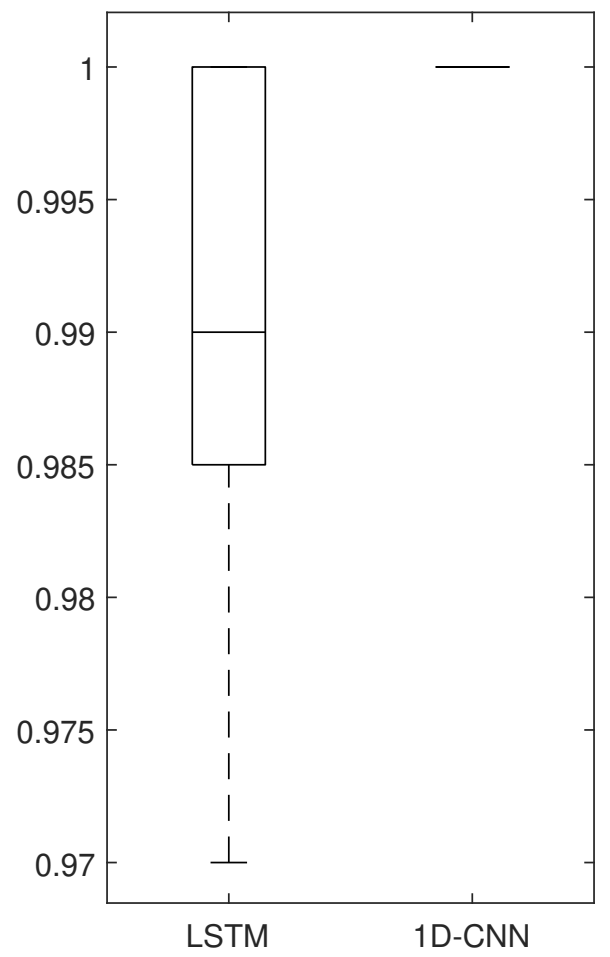

(b)

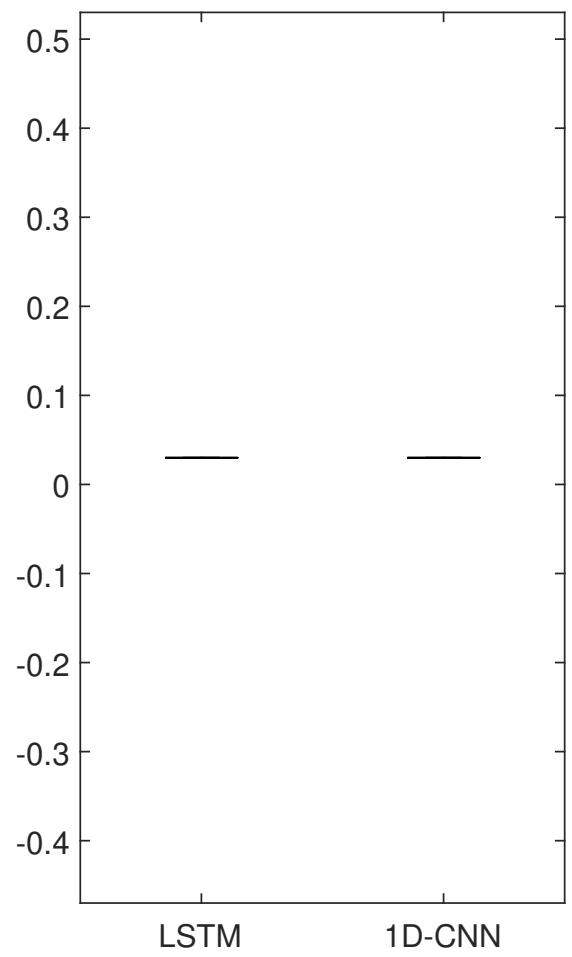

Figure 6.7: Performance evaluation for random weight initialization of QUGS data: (a) Accuracy, and (b) FNR.

One possible explanation for the perfect performance behavior of the machine learning models is based on the nature of the acceleration signals. The damages in the joints of the QUGS are highly localized, and the sensors are placed at the exact locations of damage. Furthermore, the controlled experimental laboratory setup makes the acquired signals of high quality and noise free. These properties lead to acceleration signals that have distinct, discriminating patterns between the damaged versus undamaged cases. The 1D CNN's capability to learn local structure in the time signals, and the LSTM's capability to learn long-term irregular dependencies lead to both models learning patterns that give excellent performance. 
In the proposed method, the damage classification of a time-series is obtained by voting on the classification of individual windows that constitute the entire time-series. It is observed that this voting process increases performance metrics on time-series predictions considerably in contrast to the predictions on individual windows, as illustrated by the ROC and precision-recall (PR) curves in Fig. 6.8. The ROC curve is closer to the upper left corner in the voted series predictions (Fig. 6.8. (b)) than on the individual window predictions (Fig. 6.8. (a)), which represent an increase in ROC-AUC from 0.95 to 1.0. The PR curve also shifts to the upper right corner as shown in Fig. 6.8 (d), with an increase in PR-AUC from 0.52 for voted series predictions to 0.99 for individual window predictions. It can be concluded that voting on windows decreases the probability of error significantly to obtain the final prediction. 
(a)

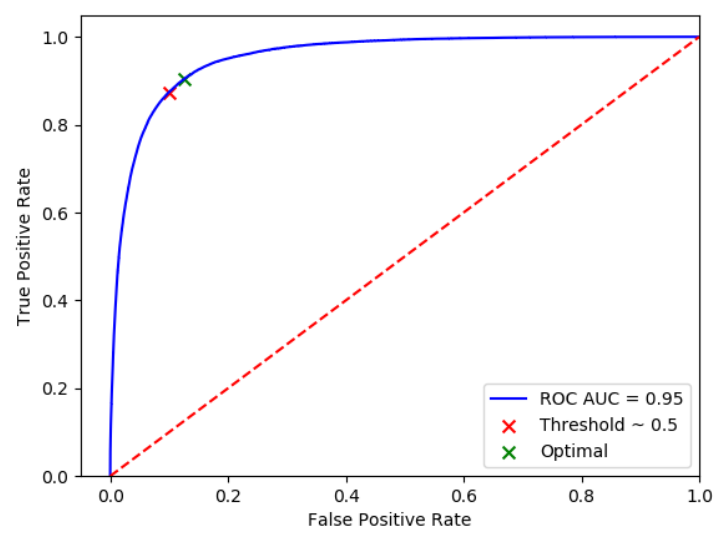

(c)

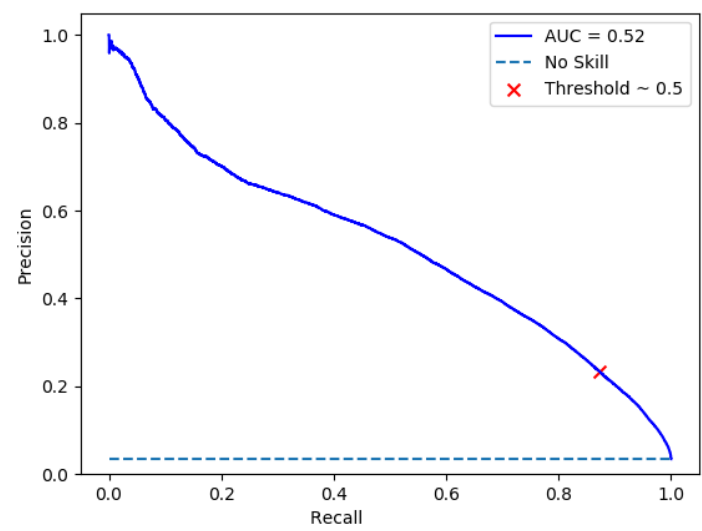

(b)

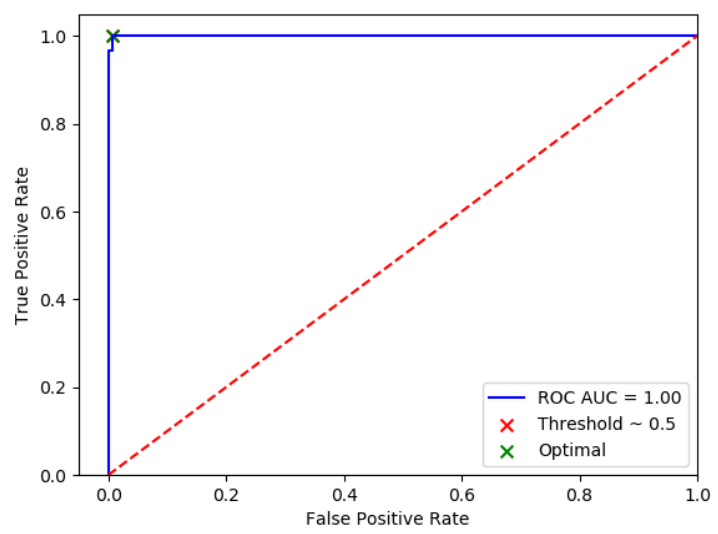

(d)

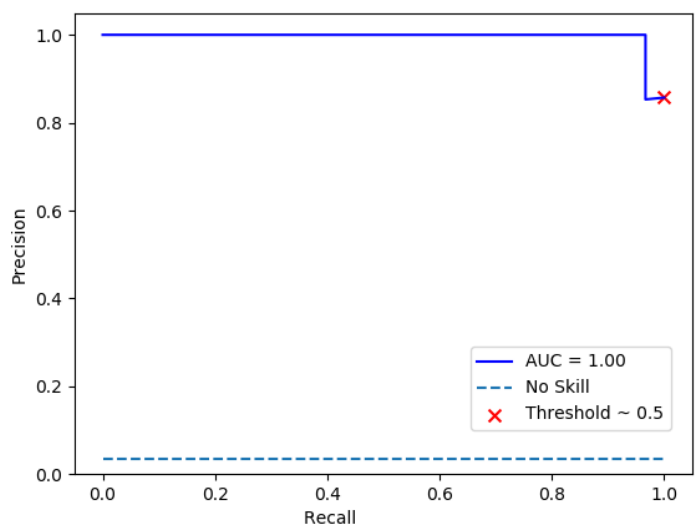

Figure 6.8: Performance of the LSTM model on the QUGS data: (a) ROC in individual windows, (b) ROC in voted series, (c) PR in individual windows, (d) PR in voted series.

Damage localization is performed in the QUGS data using Algorithm 5. In each damage scenario, a single joint out of the 30 joints in the grandstand setup was damaged by loosening of the bolts. Acceleration signals are acquired from all joints, and the proposed localization method gives damage probabilities for each joint location. The distributions of damage probabilities for multiple damage scenarios are presented as the heatmaps in Fig. 6.9. 
(a)

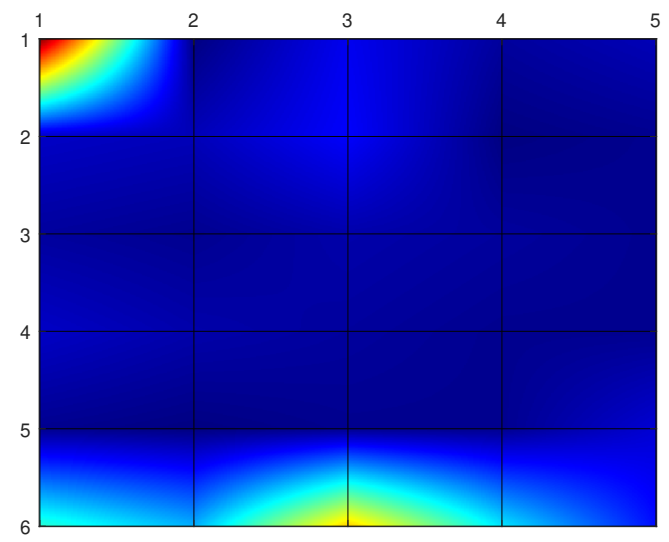

(c)

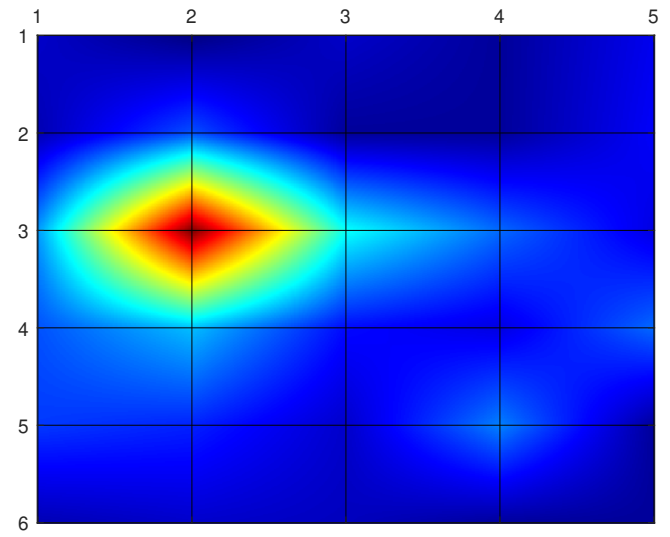

(e)

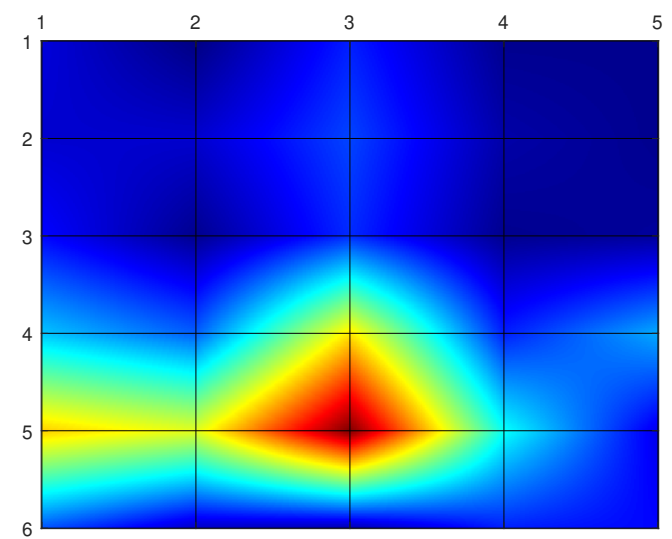

(b)

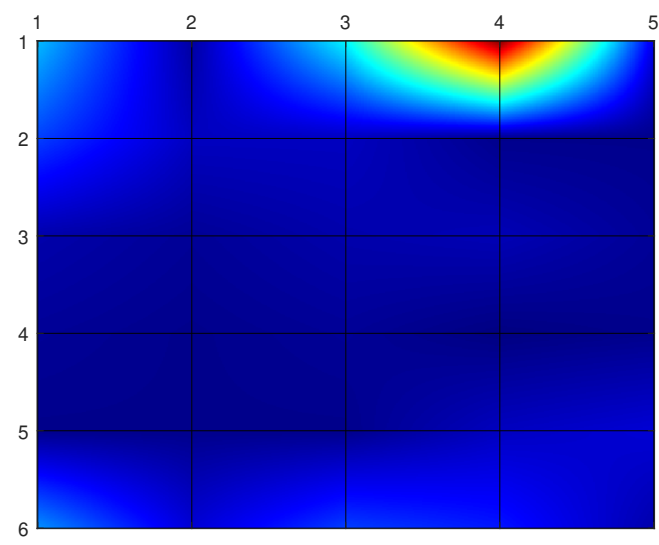

(d)

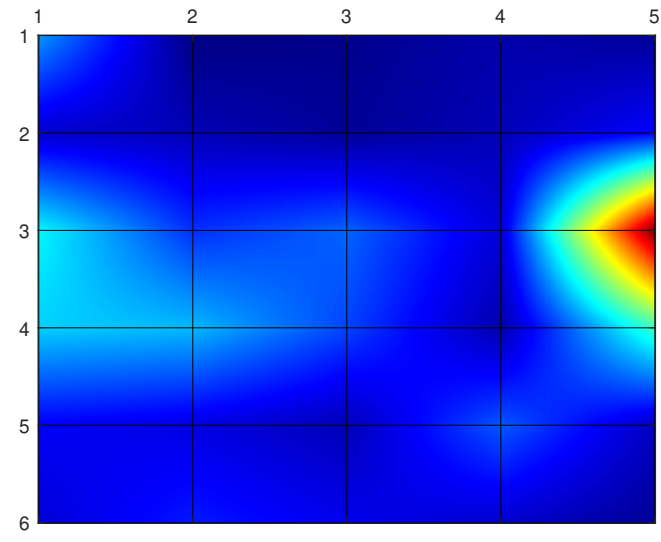

(f)

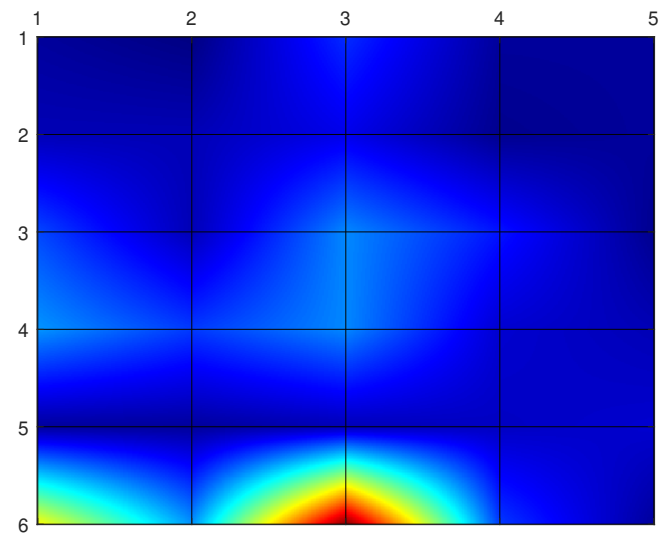

Figure 6.9: Damage localization probabilities in the QUGS data for scenarios where the damaged joint is: (a) Joint 1 [1,1], (b) Joint 4 [4,1], (c) Joint 12 [2,3], (d) Joint $15[5,3]$, (e) Joint $23[3,5]$, and (f) Joint $28[3,6]$. 
Note that the blue color indicates $P($ Damage $) \approx 0$ and red color represents $P($ Damage $) \approx$ 1. For example, Fig. 6.9 (a) shows the scenario where joint 1 of the structure is damaged as shown in Fig. 6.4 for an illustration of the numbered joints. The damage appears to be heavily localized at the joint. Fig. 6.9 (e) shows the scenario where joint 23 (location: $[3,5])$ is damaged, and it is clear that the damage is spread out as it is surrounded by 4 connected branches signifying affecting damage after loosening of the bolts.

\subsubsection{Full-scale study}

The Z24 bridge data that was used in Sections 4.4 and 5.3, is explored to illustrate the proposed method. The details of this dataset can be found from the previous sections. Several progressive damage scenarios were considered for vibration-based damage identification. Each damage scenario has multiple level of damage. All these damage scenarios are compared with the baseline undamaged state. It can be observed that each damage scenario have different classes of damage, and they were chosen to evaluate the performance of the proposed method to classify various multiclass and multilevel damage cases. For example, rupture of tendons have three levels and lowering of pier have four levels, and together they make a case of two separate damage classes. For detailed explanation of how

the damages were induced to the bridge, readers are suggested to referred to (Roeck and Teughels 2004). Multiclass problem is considered based on the type and level of damage. The reference, undamaged condition is considered as class-zero for all the cases and the other damages were assigned classes starting from 1 to $n$ depending upon the level of damage, as shown in Table 6.5. For example, in the case of rupture of tendons, the damage 
level classes 1, 2 and 3 correspond to damages induced by rupture of two tendons, then four, and finally six tendons. Similarly, there are four damage level classes for lowering of the pier.

Table 6.5: Multiclass problem description for two damage scenarios along with the class label.

\begin{tabular}{ccc} 
Problem & Damage scenario & Class label \\
\hline \hline 0 & Undamaged & 0 \\
\hline \multirow{2}{*}{1} & Rupture of 2 tendons & 1 \\
& Rupture of 4 tendons & 2 \\
& Rupture of 6 tendons & 3 \\
\hline \multirow{2}{*}{2} & Lowering of pier, $20 \mathrm{~mm}$ & 1 \\
& Lowering of pier, $40 \mathrm{~mm}$ & 2 \\
& Lowering of pier, $80 \mathrm{~mm}$ & 3 \\
& Lowering of pier, $95 \mathrm{~mm}$ & 4 \\
\hline
\end{tabular}

Optimal hyperparamters of the LSTM model on the Z24 bridge dataset are obtained by performing a random search. Table 6.6 shows the hyperparameter configuration with highest accuracy. It can be observed that LSTM performed well with $w=128$. An analysis is performed to understand the effect of window size $w$ on performance $P_{m}$. The results are illustrated in Fig. 6.10, which shows that optimal performance is achieved at $w=128$, with highest ROC-AUC and accuracy, and lowest FNR. In case of Fig. 6.10 (a), the ROCAUC and accuracy reduce while FNR increases, similarly, in case of Fig. 6.10(b) the FNR remains consistent after $w=512$ and other metrics are at their peak. Due to larger $w$, the data size reduces per damage class and it leads to overfitting of the model on the data. A 
similar random search of hyperparameters is conducted to find optimal parameters for the 1D CNN model. Five repetitions of training and testing sessions are performed to verify the robustness of the models against random initialization of network weights. Accuracy and FNR are computed for each session, and the results are shown in Fig. 6.11.

Table 6.6: Optimal configuration of the LSTM hyperparameters for the Z24 bridge benchmark data.

\begin{tabular}{ll}
\hline Parameter & Values \\
\hline \hline Window size & 128 \\
No. of windows in a sequence & 16 \\
No. of hidden layers & 3 \\
Architecture & {$[128,64,32,5]$} \\
Learning rate & 0.001 \\
Batch size & 512 \\
Training epochs & 100 with early stopping \\
\hline
\end{tabular}

(a)

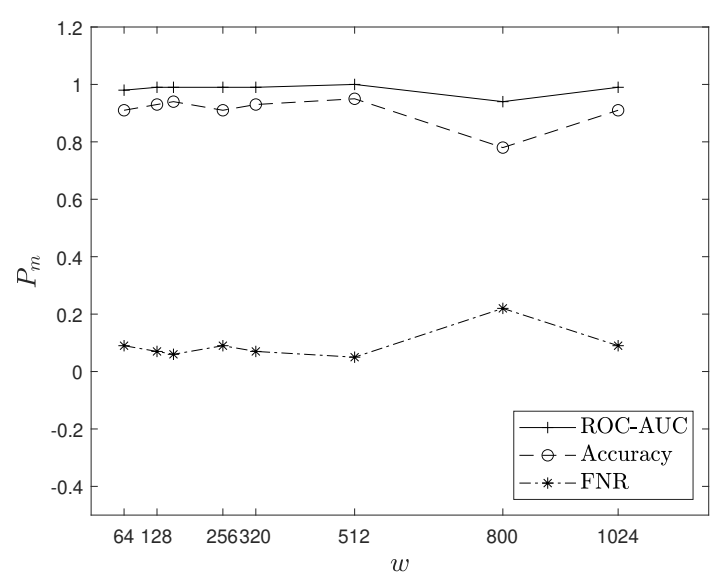

(a)

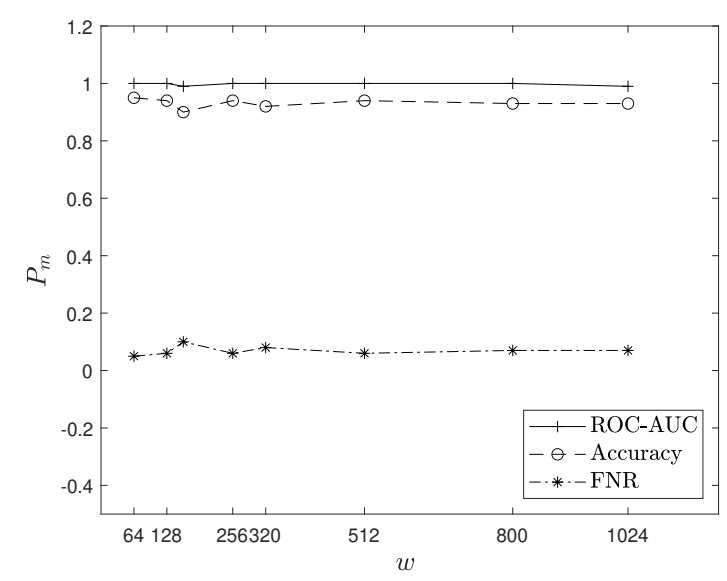

Figure 6.10: Performance evaluation of the proposed LSTM model based on window size for (a) rupture of tendons, and (b) pier settlement. 
It can be observed that for pier settlement which was measured using 4-sensors for data acquisition, and associated with 4 damage levels, the damage is considered as localized around these sensors. The LSTM performs well with highest accuracy and lowest FN rate in comparison to 1D CNN as seen in Fig. 6.11 (a), and (b). In case of rupture of tendons, where the damage is fairly distributed through out the bridge deck. The damaged signals are not highly distinguishable in comparison to undamaged signal and other severe damage measurements such as lowering of piers. The accuracy of LSTM drops to 0.9 and FN rate increases to 0.2 . However, LSTM still performs better than $1 \mathrm{D}$ CNN, as shown in Fig. 6.11 (c), and (d). It can be further emphasized that the superior performance of LSTM is attributed to its capability to learn long-term irregular dependencies of complex time signals, whereas 1D CNN learns prominently the local neighborhood structure of the signals.

(a)

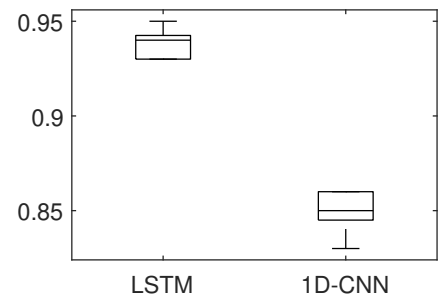

(c)

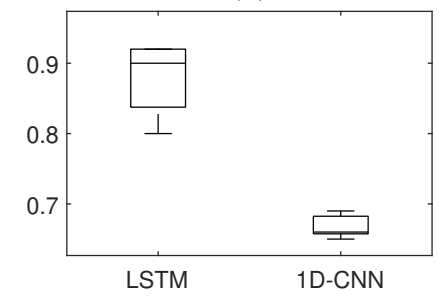

(b)

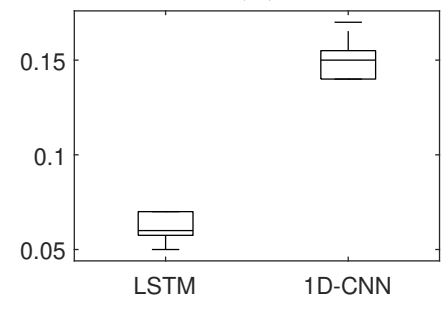

(d)

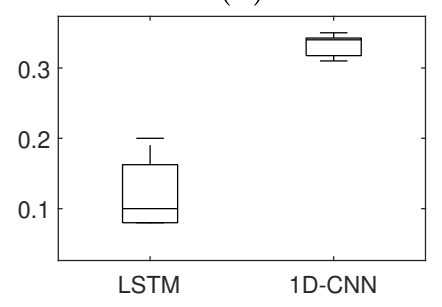

Figure 6.11: Performance evaluation of LSTM for random weight initialization for various damage cases in the Z24 bridge: (a) accuracy for pier settlement, (b) FNR for pier settlement, (c) accuracy for rupture of tendons, (d) FNR for rupture of tendons. 
Finally, the optimal parameters are used to evaluate the performance of proposed method on full-series versus voted-windowed samples. It is observed that voting on windowed dataset increases accuracy considerably and it is evident in ROC-AUC and precisionrecall (PR)-AUC curves, as presented in Fig. 6.12, and 6.13, respectively. It can be observed that voting on windows from non-localized signal increases the probability considerably by allocating the majority class and ignoring the non-prominent class along with increasing the data samples per class. As shown in Fig. 6.12, voting on individual windows has improved both ROC-AUC and PR-AUC. However, due to the localized measurement acquisition, and severity of damage in pier settlement, the difference in AUCs of various cases was comparatively similar to the QUGS damage scenario. Moreover, as observed in Fig. 6.13, where the damage was considerably distributed in case of rupture of tendons, voting on windows increased the ROC-AUC by $5 \%$ and PR-AUC by $13 \%$. 
(a)

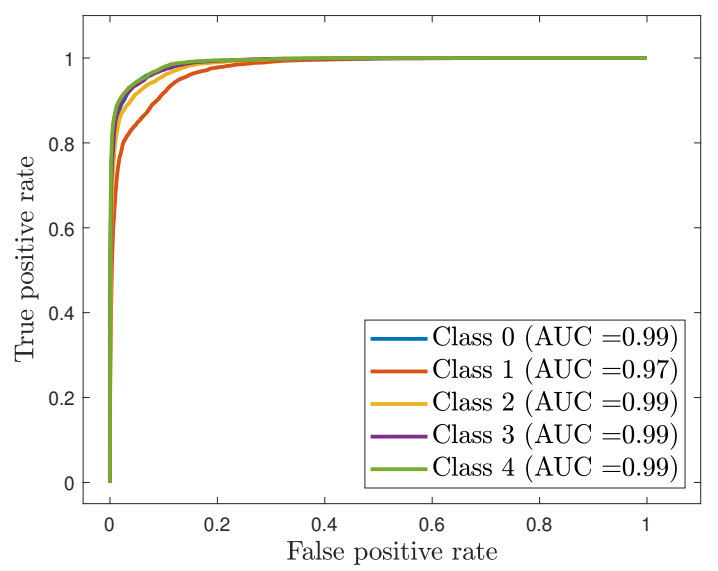

(c)

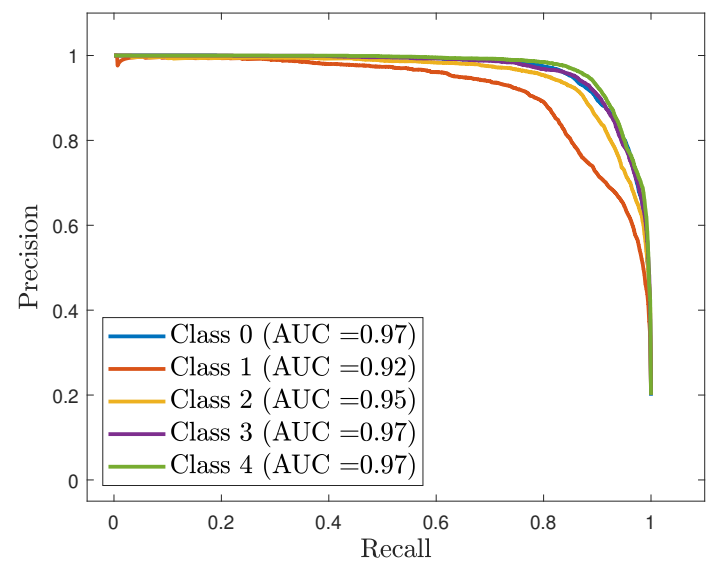

(b)

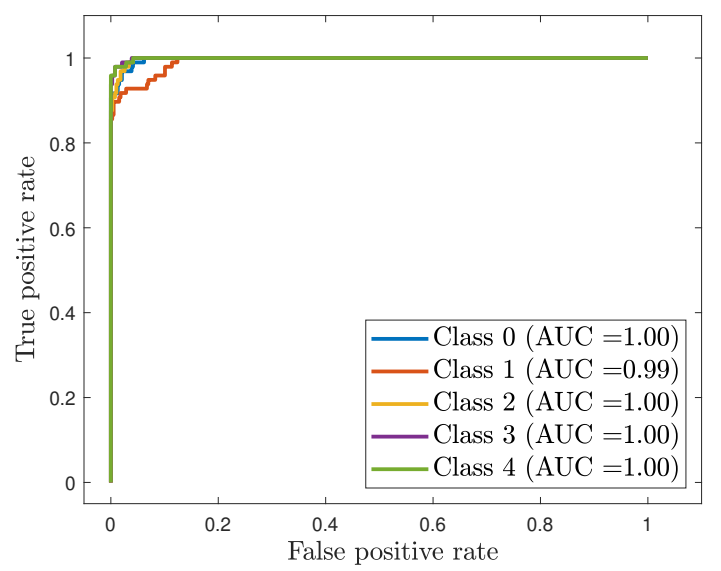

(d)

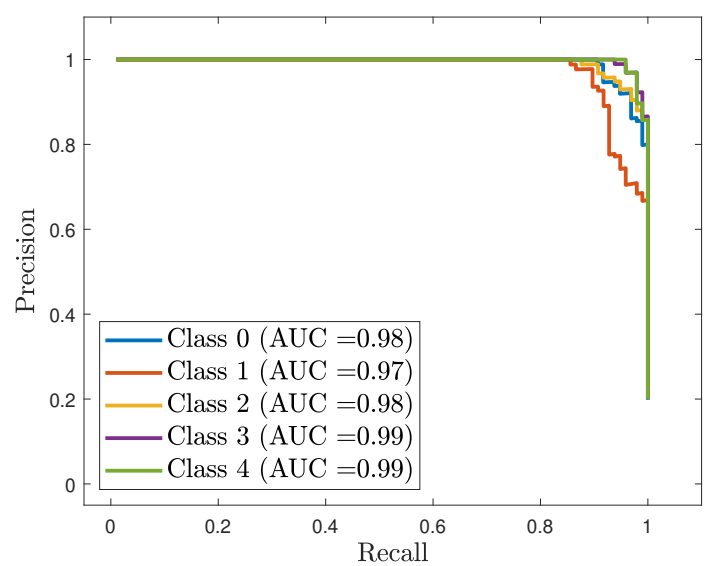

Figure 6.12: Performance of the proposed LSTM model in the Z24 bridge for pier settlement: (a) ROC in individual windows, (b) ROC in voted series, (c) PR in individual windows, (d) PR in voted series. 
(a)

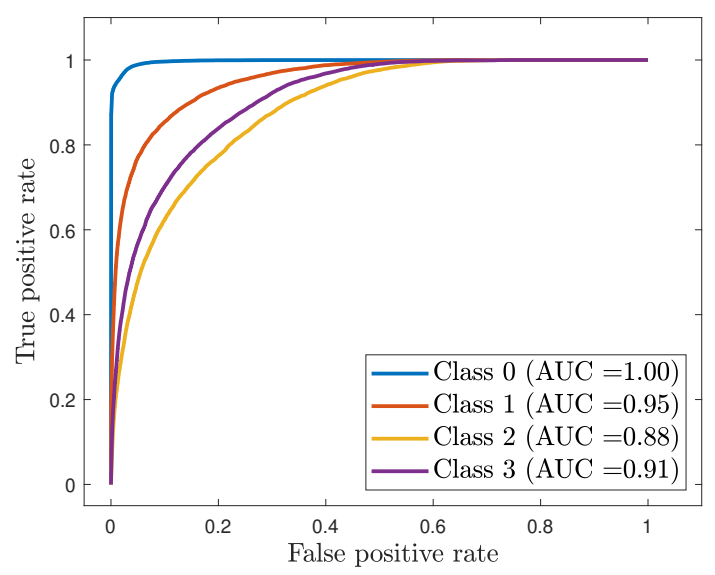

(c)

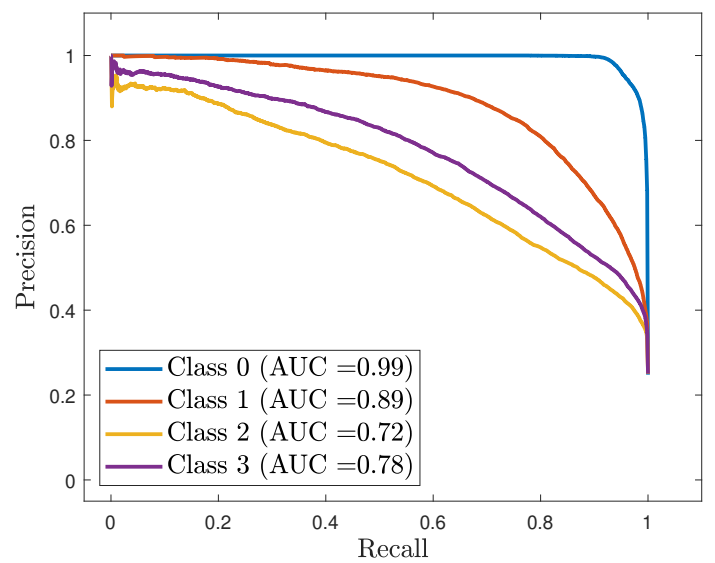

(b)

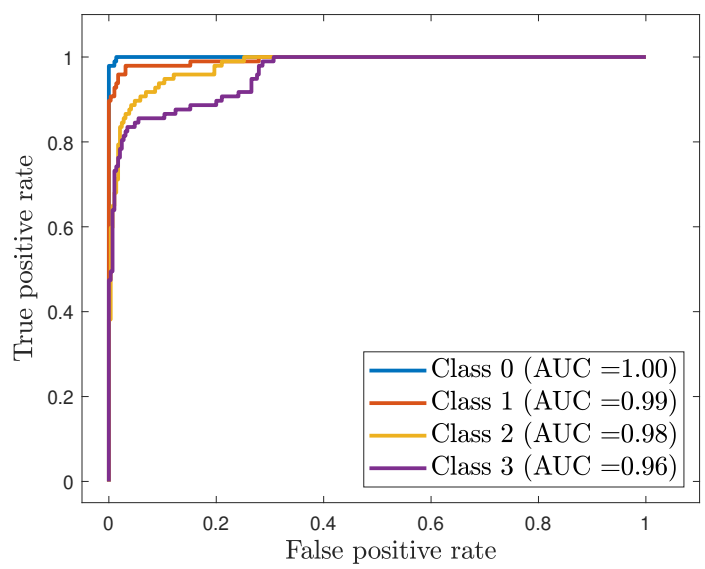

(d)

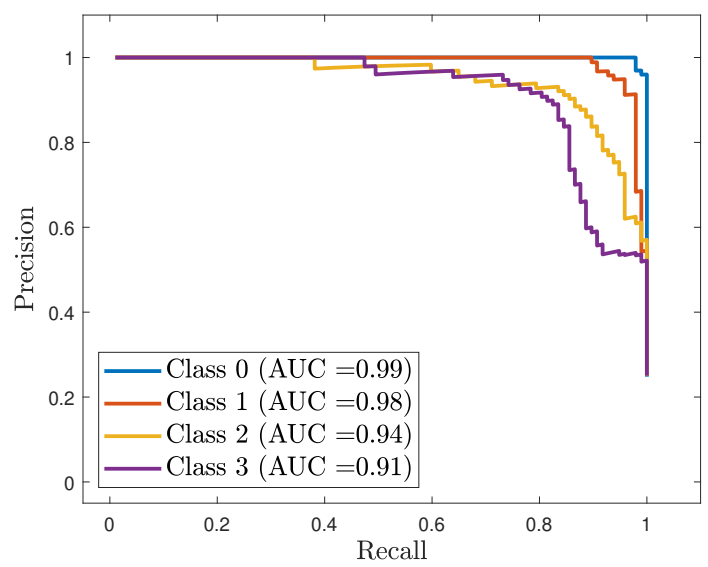

Figure 6.13: Performance of the proposed LSTM model by windowing the data of the Z24 bridge in case of rupture of tendons: (a) ROC in individual windows, (b) ROC in voted series, (c) PR in individual windows, (d) PR in voted series.

Damage localization is performed using Algorithm 5 for multilevel and multiclass damage scenarios of lowering of pier and rupture of tendons. In these damage scenarios, the damage is not highly localized as in the experimental study of the previous section. Three different structural components of the bridge are used to localize damage and understand the effect of pier settlement. An undamaged pier in Utzenstorf, bridge deck, and damaged pier in Koppigen are used to represent the predicted probability $\left(P_{p}\right)$ and infer damages in three components. The Koppigen pier is used for inducing the damage by lowering it 
in several increments starting with $20 \mathrm{~mm}, 40 \mathrm{~mm}, 80 \mathrm{~mm}$, and moving to $95 \mathrm{~mm}$ at the last stage. The $P_{p}$ is plotted against the sensor number and a dash-dotted average of $P_{p}$ of structural component is shown as a representation of combined $P_{p}$ for corresponding structural component, as shown in Fig. 6.14. For example, Fig. $6.14(a, b, c)$ represents $P_{p}$ for undamaged pier (UDP), bridge deck (BD), and damaged pier (DP) for $20 \mathrm{~mm}$ lowering of piers, respectively. Similarly, Figs. $6.14(d, e, f)$ and $(g, h, i)$ show $P_{p}$ for $40 \mathrm{~mm}$ and 85 mm lowering of piers, respectively.

Due to non-localization of measurement acquisition, it is difficult to infer damage location while considering each sensor separately. However, it is possible to compare average $P_{p}$ of each structural component for various damage cases. The results considering average $P_{p}$ for each structural component and various damage levels are shown in Fig. 6.15. Although there is no correlation between $P_{p}$ and damage severity, however, as the severity increases, the signals become more distinguishable and the proposed LSTM model performs the classification more effectively. It can be observed from Fig. 6.15 that UDP shows lowest predicted probability due to its similarity to undamaged baseline signal, however, both BD and DP show higher prediction accuracy. The reason for bridge deck's highest probability of damage is attributed to the surface area and larger affect of differential pier-settlement in entire bridge system. The bridge suffers higher changes in structural responses (i.e., deflection, bending moment and shear) than at damaged pier itself, as it acted as a support. 
(a)

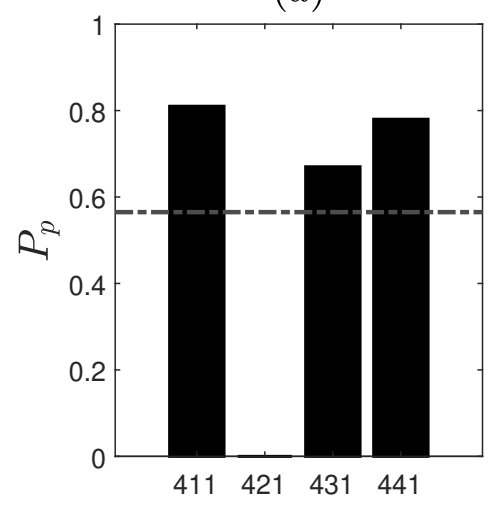

(d)

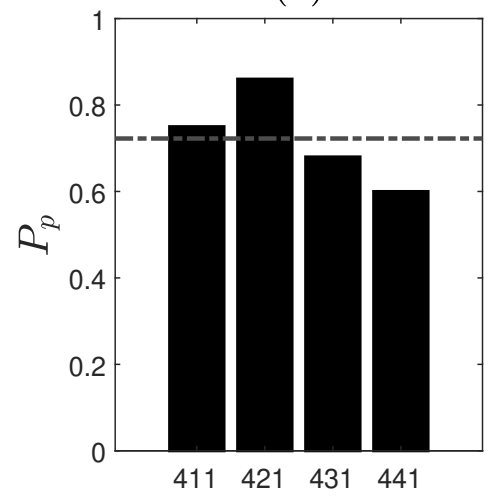

(g)

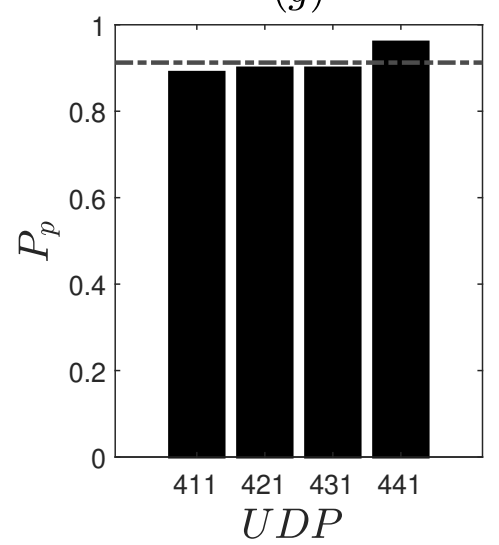

(b)

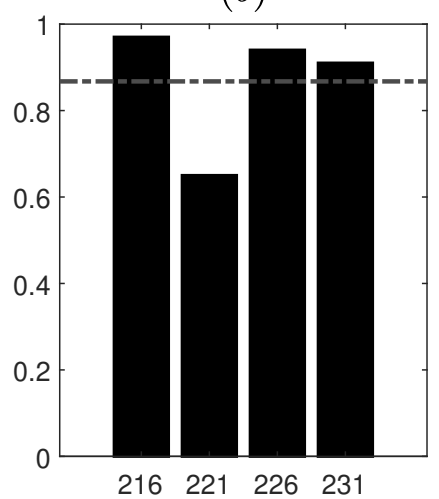

(e)

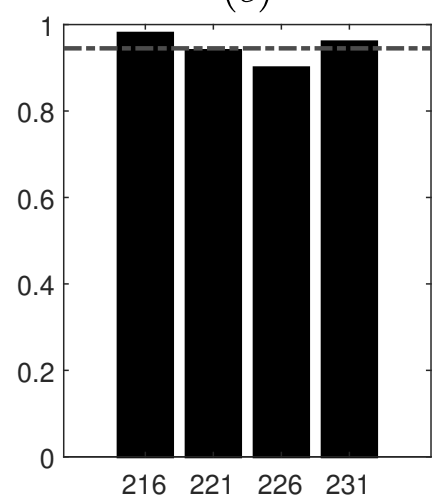

$(h)$

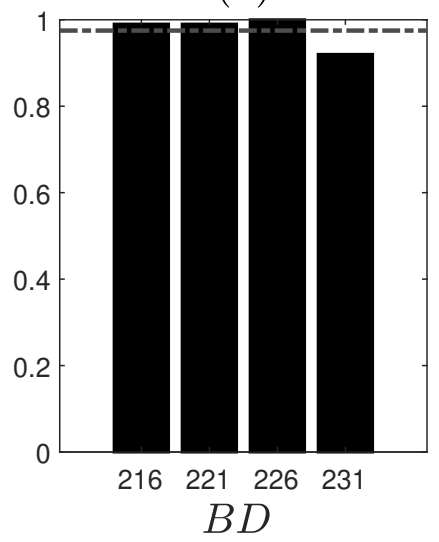

(c)

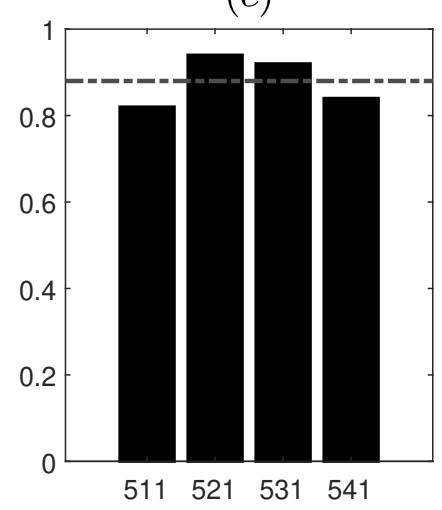

(f)

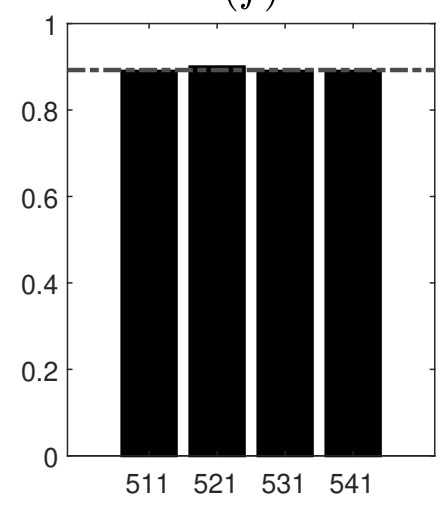

(i)

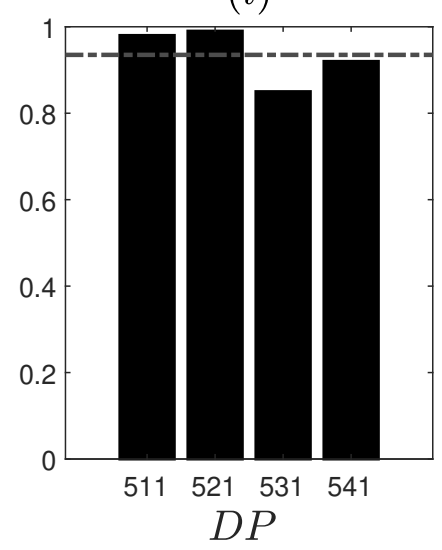

Figure 6.14: Damage localization for lowering of pier for three damage levels, where, $(a, b, c)$ are for $20 \mathrm{~mm}$ lowering of piers, $(d, e, f)$ are for $40 \mathrm{~mm}$ lowering of piers, $(g, h, i)$ are for $95 \mathrm{~mm}$ lowering of piers. 


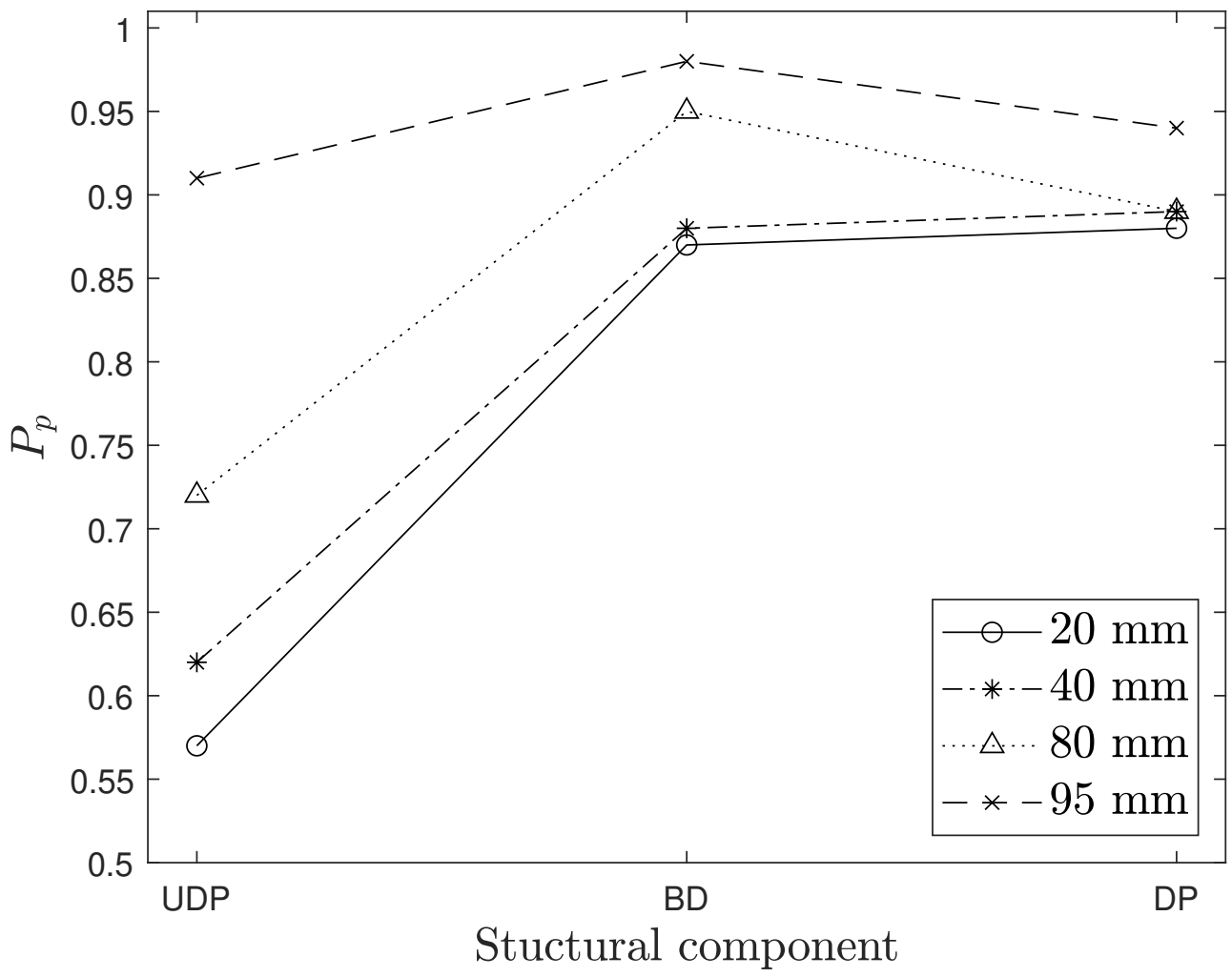

Figure 6.15: Damage localization for lowering of pier, where legend shows the amount of pier-settlement.

Similarly, for rupture of tendons, the most affected area would be the bridge deck and the damage induced due to rupture of tendons will create a non-localized and distributed damage throughout the bridge deck in comparison to bridge piers. The damage localization per sensors is avoided due to non-conclusive inference and a comparison between structural components of the bridge is provided directly in Fig. 6.16. It can be observed that rupture of 6 tendons prove to be worse damage level scenario in comparison to the ruptures with 2 and 4 tendons. 


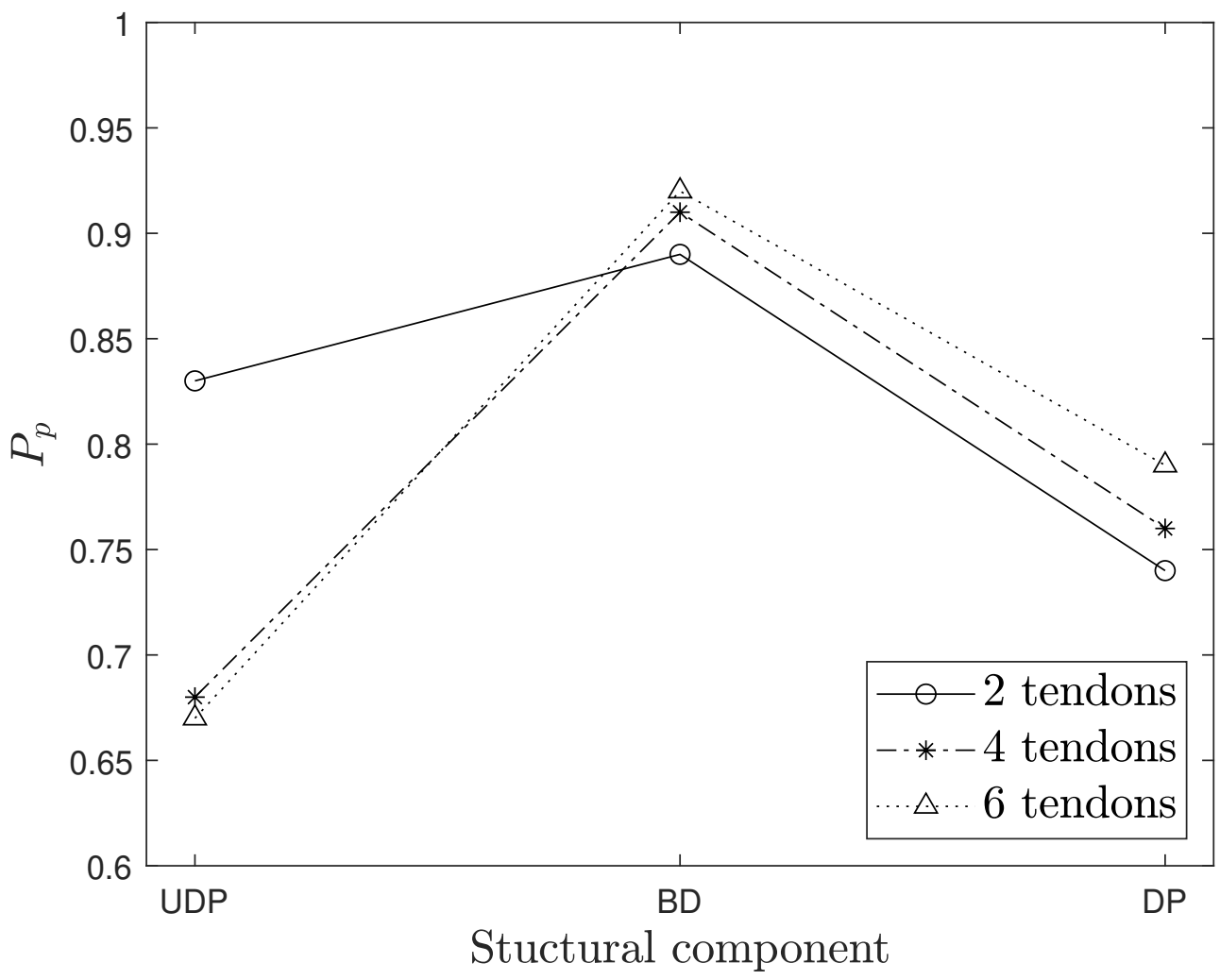

Figure 6.16: Damage localization for rupture of tendons, where the legend shows the number of tendon ruptures.

\subsection{Summary}

In this chapter, damage localization using a windowed-LSTM based deep learning algorithm is employed for multiclass and multilevel damage detection. Various types of damages starting from binary to a maximum of five classes were classified into multiclass damage level. Limited dataset is augmented using windowing of the time-series measurements and the prediction accuracy is improved by novel voting approach on windowed data. It is observed that the proposed algorithm performs well with nonlocalized and irregular sample sizes, and learns the long-term dependencies. The proposed algorithm is analyzed with 
sensitivity analysis on window-size as the external parameter to the model. A parametric study is also presented for random initialization of weights. The accuracy improvement of the proposed algorithm is illustrated through a comparison between a single series dataset and windowed-voted for ROC and precision-recall AUC. In this chapter, it is demonstrated that a simple LSTM architecture is capable of classifying the time-series signals into multiclass and multidamage levels with high accuracy.

Traditional unsupervised methods rely on the appropriate selection of model orders and extraction of useful features that involved significant user discretion for a large-scale civil structure. On the other hand, the proposed deep learning model based on sequential data is independent of any feature selection process and offers robust, accurate and autonomous approaches to complex damage localization. Like any other supervised techniques, the LSTM method also requires a significant amount of training data to classify and predict the damage. With the recent advancement of remote and autonomous sensors and internetof-things, long-term SHM technologies have shown significant promise to monitor critical infrastructure in smart cities (Talari et al. 2017). Such long-term and real-time monitoring allow the SHM researchers and practitioners to provide low-cost periodical SHM data with multiclass health conditions of the structures (Mishra et al. 2020), serving as the potential training data for the deep learning techniques, such as, CNN and LSTM. 


\section{Chapter 7}

\section{Key Conclusions and Discussions}

In this chapter, the key conclusions, thesis contributions and the potential future work are summarized.

\subsection{Key Conclusions}

- Damage detection based on frequency change is explored using a hybrid TF method by integrating MEMD with SST. The proposed method is shown to have the capability of separating modal responses and, subsequently, identifies the time-varying behavior of the structure with both discrete and progressive damages. The proposed method's efficacy is validated under noise and several damage cases using numerical and full-scale studies. While integrating SST with MEMD, it shows possibilities of MEMD to perform damage detection using multichannel measurements.

- A basis-free MEMD-based damage localization method is introduced using limited 
sensor measurements. The decomposed mono-component modal responses are further used to evaluate the modal energy to derive the damage localization features from the limited sensors. The proposed method works well on various damage scenarios and is effective in localizing the damage. The proposed method is capable of identifying as minimal as $2 \%$ change in global modal parameters of structures, outperforming the existing TF methods to delineate the minor global damage. The limited measurement aspect of damage localization is explored by selecting fewer sensors, and it is shown that with limited measurements, the proposed method is as effective as the total number of measurements equals the degree of freedom of the model.

- A variant of popular deep learning algorithm CNN, 1D CNN, is employed to cater one-dimensional time-series data for the classification task. A windowed-1D CNN is employed using limited datasets for multiclass damage identification and localization. The limited dataset is augmented using the vibration data windowing, and a novel voting approach improves the prediction accuracy on windowed classes. The robustness of the proposed algorithm is shown using various parametric and sensitivity analysis. The improvement in the accuracy is illustrated through a comparison between a single series dataset and windowed-voted time-series for ROC and precision-recall AUC. It is demonstrated that a simple 1D CNN architecture with only one hidden layer is capable of classifying the time-series of vibration data into multiclass with high accuracy.

- The performance of 1D CNN is further improved by introducing a windowed-LSTM based deep learning algorithm for multiclass and multilevel damage. It is observed 
that the proposed algorithm performs well with non-localized and irregular sample sizes and learns the long-term dependencies. The accuracy of the proposed algorithmis illustrated through a comparison between a single time-series dataset and windowed-voted for ROC and precision-recall AUC. The proposed LSTM architecture can classify the time-series signals into multiclass and multi damage levels with high accuracy.

\subsection{Thesis Contributions}

The research conducted through this thesis is directed towards creating an autonomous VDD with minimal to no-user intervention. Advanced basis-free TF methods, MEMD, and deep learning-based 1D CNN, and LSTM networks are explored to provide a framework capable of detecting and localizing damages in structures. The broad contributions of this thesis are mentioned below:

- A basis-free TF method capable of localizing various damage cases is introduced to evaluate its performance under the various severity and duration of the damage. A critical issue of limited sensors is attempted to solve by using a fewer number of sensors to identify and localize the damage.

- Deep learning algorithms are popular for learning from the data and provide a framework for identifying the new data. For the first time, 1D CNN is introduced through this thesis for multiclass damage identification and localization in civil structures.

- An improvement to 1D CNN, LSTM based VDD framework is introduced for mul- 
ticlass damage classification and localization. The proposed method can provide an autonomous SHM framework for large-scale engineering structures.

\subsection{Future Work}

The proposed research can further be extended to assess the performance of degrading structures.

- In the current research, TF modal decomposition method is explored to localize damage using the non-parametric feature. It is worth to introduce new non-parametric features that can collaboratively be used to push the envelope of structural damage localization in SHM. Feature Engineering is at the forefront of developing handcrafted features to understand the dynamics of data and can be implemented in the context of SHM.

- SHM instrumentations are often conducted for a short duration; however, while using deep neural networks, data availability is crucial to effectively assess the health of a structure. It is crucial to acquire as much as possible data from various structures to efficiently use a broad range of deep learning algorithms for precise damage diagnosis frameworks. There is a need to acquire data for multiclass data from large scale structures. The data can further be acquired using modern sensors such as cameras, mobile devices, and drones. These datasets in the form of TF images of videos (a stream of images) can be used with any deep learning algorithm with minimal changes. To facilitate the application of SHM and make the algorithms suitable 
for smart infrastructure and cities, it is crucial to introduce cyber-physical SHM systems with handheld devices capable of conducting continuous monitoring of the civil infrastructure.

- There is a continuous debate on the use of unsupervised versus supervised learning approach for damage diagnosis. Both methods have their own merits and demerits. It would be highly beneficial for the SHM community to combine both and explore self-supervised algorithms, that can work with considerably less labeled data, leading to a significant faster SHM framework.

- Damage prognosis in civil structures is often considered a difficult task due to the non-availability of the remaining useful life data. However, Reinforcement Learningbased AI methods can solve and create an autonomous framework for remaining useful life evaluation that can be explored in the future. 


\section{References}

[1] Abazarsa, F., Ghahari, S., Nateghi, F., and Taciroglu, E. (2013). Response-only modal identification of structures using limited sensors. Structural Control and Health Monitoring, 20:987-1006.

[2] Abdeljaber, O. and Avci, O. (2016). Non-parametric structural damage detection algorithm for ambient vibration response: utilizing artificial neural networks and selforganizing maps. Journal of Architectural Engineering, ASCE, 22(2):04016004.

[3] Abdeljaber, O., Avci, O., Kiranyaz, M., Boashash, B., Sodano, H., and Inman, D. (2018). 1-d cnns for structural damage detection: Verification on a structural health monitoring benchmark data. Neurocomputing, 275:1308-1317.

[4] Abdeljaber, O., Avci, O., Kiranyaz, S., Gabbouj, M., and Inman, D. (2017). Real-time vibration-based structural damage detection using one-dimensional convolutional neural networks. Journal of Sound and Vibration, 388:154-170.

[5] Abdulkarem, M., Samsudin, K., Rokhani, Z., and Rasid, A. (2019). Wireless sensors network for structural health monitoring: A contemporary review of technologies, challenges, and future direction. Structural health monitoring, 19(3):693-735. 
[6] Alamdari, M., Rakotoarivelo, T., and Khoa, N. (2017). A spectral based clustering for structural health monitoring of the sydney harbour bridge. Mechanical Systems and Signal Processing, 87:384-400.

[7] Almasri, N., Sadhu, A., and Ray Chaudhuri, S. (2020). Toward compressed sensing of structural monitoring data using discrete cosine transform. Journal of Computing in Civil EngineeringASCE, 34(1).

[8] Amezquita-Sanchez, J. and Adeli, H. (2016). Signal processing techniques for vibrationbased health monitoring of smart structures. Archives of Computational Methods in Engineering, 23(1):1-15.

[9] Amini, F. and Hedayati, Y. (2016). Underdetermined blind modal identification of structures by the earthquake and ambient vibration measurements via sparse component analysis. Journal of Sound and Vibration, 366:117-132.

[10] Amini, F. and Karami, K. (2012). Damage detection algorithm based on identified system markov parameters (dda/ismp) in building structures with limited sensors. Smart Materials and Structures, 21(5):055010.

[11] An, Y., Chatzi, E., Sim, S.-H., Laflamme, S., Blachowski, B., and Ou, J. (2019). Recent progress and future trends on damage identification methods for bridge structures. Structural Control and Health Monitoring, 26(10):e2416.

[12] Andria, G., Savino, M., and Trotta, A. (1994). Application of wigner-ville distribution to measurements on transient signals. IEEE transactions of instrumentation and measurement, 43(2):187-193. 
[13] ASCE (2017). American Infrastructure Report Card, GPA: D+, https://www.infrastructurereportcard.org.

[14] Auger, F., Flandrin, P., Lin, Y.-T., McLaughlin, S., Oberlin, T., Wu, and H.-T. (2013). Recent advances in time-frequency reassignment and synchrosqueezing. IEEE Signal Processing Magazine, 30:32-41.

[15] Avci, O., Abdeljaber, O., Kiranyaz, S., Hussein, M., Gabbouj, M., and Inman, D. (2021). A review of vibration-based damage detection in civil engineering structures: from traditional methods to machine learning and deep learning based applications. Mechanical Systems and Signal Processing, 147:107077.

[16] Azimi, M. and Pekcan, G. (2020). Structural health monitoring using extremely compressed data through deep learning. Computer-Aided Civil and Infrastructure Engineering, 35(6):597-614.

[17] Bagheri, A., Hosseinzadeh, A., Z., R., P., and Amiri, G. (2016). Time domain damage localization and quantification in seismically excited structures using a limited number of sensors. Journal of Vibration and Control, 23(8):2942-2961.

[18] Bakhary, N., Hao., H., and Deeks, A. (2010). Structural damage detection using neural network with multi-stage sub-structuring. Advances in Structural Engineering, 13(1):95-110.

[19] Bao, X., Fan, T., Shi, C., and Yang, G. (2020). One-dimentional convolutional neural network for damage detection of jacket-type offshore platforms. Ocean Engineering, 108293. 
[20] Bao, Y., Tang, Z., Li, H., and Zhang, Y. (2019). Computer vision and deep learningbased data anomaly detection method for structural health monitoring. Structural Health Monitoring, 18(2):401-421.

[21] Barbosh, M., Singh, P., and Sadhu, A. (2020). Empirical mode decomposition and its variants: A review with applications in structural health monitoring. Smart Materials and Structures," accepted.

[22] Bergstra, J. and Bengio, Y. (2012). Random search for hyper-parameter optimization. Journal of Machine Learning, 13:281-305.

[23] Bernal, D. (2010). Load vectors for damage location in systems identified from operational loads. Journal of Engineering Mechanics, 136(1):31-39.

[24] Brockwell, P. and Davis, R. (1991). Time series theory and methods. Springer, New York.

[25] Cao, S. and Ouyang, H. (2017). Robust structural damage detection and localization based on joint approximate diagonalization technique in frequency domain. Smart Materials and Structures, 26(1):015005.

[26] Carden, E. and Brownjohn, J. (2008). Arma modelled time-series classification for structural health monitoring of civil infrastructure. Mechanical Systems and Signal Processing, 22(2):295-314.

[27] Casciati, F., Faravelli, L., and Borghetti, F. (2003). Wireless links between sensordevice control stations in long-span bridges. In Smart Structures and Materials: Smart Systems and Nondestructive Evaluation, volume 5057, page 1-7, San Diego, CA. 
[28] Castiglione, R., Antoni, J., and Garibaldi, L. (2018). Separation and identification of structural modes in largely underdetermined scenarios using frequency banding. Journal of Sound and Vibration, 414:192-217.

[29] Cawley, P. (2018). Structural health monitoring: Closing the gap between research and industrial development. Structural health monitoring, 17(5):1225-1244.

[30] Cha, Y.-J., Choi, W., and Buyukozturk, O. (2017). Deep learning-based crack damage detection using convolutional neural networks. Computer-Aided Civil and Infrastructure Engineering, 32(5):361-378.

[31] Cha, Y.-J., Trocha, P., and Büyüköztürk, O. (2016). Field Measurement-Based System Identification and Dynamic Response Prediction of a Unique MIT Building. Sensors, 16(7):1016.

[32] Chang, K. and Chi, S. (2019). Bridge clustering for systematic recognition of damage patterns on bridge elements. Journal of Computing in Civil EngineeringASCE, 33(5):04019028.

[33] Chen, G. (2016). A gentle tutorial of recurrent neural network with error backpropagation. preprint arXiv:1610.02583.

[34] Chen, T., He, H., He, C., and Chen, G. (2019). New parameter-identification method based on qr decomposition for nonlinear time-varying systems. 145:04018118, ASCE.

[35] Cohen, L. (1995). Time frequency analysis. Prentice Hall, Englewood Cliffs, NJ. 
[36] Cortes, C. and Vapnik, V. (1995). Support-vector networks. Machine Learning, $20: 273-297$.

[37] CSCE (2019). The Canadian Infrastructure Report Card, 2019, http://canadianinfrastructure.ca/.

[38] Cury, A., Crémona, C., and Diday, E. (2010). Application of symbolic data analysis for structural modification assessment. Engineering Structures, 32(3):762-775.

[39] Daubechies, I. (1990). The wavelet transform. TF localization and signal analysis, 36(5):961-1005.

[40] Daubechies, I., Lu, J., and Wu, H. (2010). Synchrosqueezed wavelet transforms: An empirical mode decomposition-like tool. Applied and Computational Harmonic Analysis, $30(2): 243-261$.

[41] Deckermann, U., Li, J., and Samali, B. (2013). identification of member connectivity and mass changes on a two-storey framed structure using frequency response functions and artificial neural networks. Journal of Sound and Vibration, 332(16):3636-3653.

[42] Dziedziech, K., Staszewski, W., and Uhl, T. (2015). Wavelet-based modal analysis for time-variant systems. Mechanical Systems and Signal Processing, 50:323-337.

[43] Erazo, K., Sen, D., Nagarajaiah, S., and Sun, L. (2019). Vibration-based structural health monitoring under changing environmental conditions using kalman filtering. Mechanical systems and signal processing, 117:1-15.

[44] Fallahian, M., Khoshnoudian, F., and Meruane, V. (2018). Ensemble classification 
method for structural damage assessment under varying temperature. Structural Health Monitoring, 17(4):747-762.

[45] Feng, D. and Feng, M. (2018). Computer vision for shm of civil infrastructure: From dynamic response measurement to damage detection - a review. Engineering Structures, 156:105-117.

[46] Gardner, P., Fuentes, R., Dervilis, N., Mineo, C., Pierce, S., Cross, E., and Worden, K. (2020). Machine learning at the interface of structural health monitoring and nondestructive evaluation. Philosophical Transactions of Royal Society, A378:20190581.

[47] Gatti, M. (2019). Structural health monitoring of an operational bridge: A case study. Engineering Strutcures, 195:200-209.

[48] Ghiasi, R., Torkzadeh, P., and Noori, R. (2016). A machine learning approach for structural damage detection using least square support vector machine based on a new combinational kernel function. Structural Health Monitoring, 15(3):302-316.

[49] Gomes, G., Mendéz, Y., Silva Lopes Alexandrino, P., Cunha, S., and Ancelotti, A. (2018). The use of intelligent computational tools for damage detection and identification with an emphasis on composites $-A$ review. Composite Structures.

[50] Goodfellow, I., Bengio, Y., and Courville, A. (2017). Deep learning. MIT Press, Cambridge, MA.

[51] Gui, G., Pan, H., Lin, Z., Li, Y., and Yuan, Z. (2017). Data-driven support vector machine with optimization techniques for structural health monitoring and damage detection. KSCE Journal of Civil Engineering, 21(2):523-534. 
[52] Gul, M. and Catbas, F. (2010). Statistical pattern recognition for structural health monitoring using time series modeling: Theory and experimental verifications. Mechanical Systems and Signal Processing, 23(7):2192-2204.

[53] Gul, M. and Catbas, F. (2011). Structural health monitoring and damage assessment using a novel time series analysis methodology with sensor clustering. Journal of Sound and Vibration, 330(6):1196-1210.

[54] Gulgec, N., Takáč, M., and Pakzad, S. (2019). Convolutional neural network approach for robust structural damage detection and localization. Journal of Computing in Civil EngineeringASCE, 33(3):04019005.

[55] Gulgec, N., Takac, M., and Pakzad, S. (2017). Structural damage detection using convolutional neural networks". In Conference Proceedings of the Society for Experimental Mechanics Series, page 331 - 337.

[56] Guo, J., Xie, X., Bie, R., and Sun, L. (2014). Structural health monitoring by using a sparse coding-based deep learning algorithm with wireless sensor networks". Pers Ubiquit Comput, 18:-.

[57] Guo, Y. and Kareem, A. (2016). System identification through nonstationary data using time-frequency blind source separation. Journal of Sound and Vibration, 371:110-131.

[58] Hakim, S. and Razak, H. (2013a). Adaptive neuro fuzzy inference systems (anfis) and artificial neural networks (anns) for structural damage identification. Structural Engineering and Mechanics, 46(6):779-802. 
[59] Hakim, S. and Razak, H. (2013b). Structural damage detection of steel bridge using artificial neural networks and finite element models. Steel and Composite Structures, 14(4):367-377.

[60] Hazra, B., Sadhu, A., Roffel, A., Paquet, P., and Narasimhan, S. (2011). Underdetermined blind identification of structures by using the modified cross-correlation method. Journal of Engineering Mechanics, 138(4):327-337.

[61] Herrera, R., Han, J., and Baan, M. (2014). Applications of the synchrosqueezing transform in seismic time-frequency analysis. Geophysics, 79(3):55- 64 .

[62] Hochreiter, S. and Schmidhuber, J. (1997). Long short-term memory. Neural computation, 9(8):1735-1780.

[63] Hou, R. and Xia, Y. (2020). Review on the new development of vibration-based damage identification for civil engineering structures: 2010-2019. Journal of Sound and Vibration, 115741.

[64] Hou, R. and Xia, Y. (2021). Review on the new development of vibration-based damage identification for civil engineering structures: 2010-2019. Journal of Sound and Vibration, 491:115741.

[65] Hou, Z., Noori, M., and St. Amand, R. (2000). Wavelet-based approach for structural damage detection. Journal of Engineering Mechanics, 126(7):677-683.

[66] Huang, N., Shen, Z., and Long, S. (1998). The empirical mode decomposition and the hilbert spectrum for nonlinear and non-stationary time series analysis. Proceedings 
of the Royal Society of London A: Mathematical, Physical and Engineering Sciences, 454(1971):903-995.

[67] Ioffe, S. and Szegedy, C. (2015). Batch normalization: accelerating deep network training by reducing internal covariate shift. arXiv:1502.03167.

[68] Joyce, B., Dodson, J., Laflamme, S., and Hong, J. (2018). An experimental test bed for developing high-rate structural health monitoring methods. Shock and Vibration, 3827463.

[69] Kang, M.-S., Kim, N., Lee, J., and An, Y.-K. (2020). Deep learning-based automated underground cavity detection using three-dimensional ground penetration radar. Structural Health Monitoring, 19(1):173-185.

[70] Kankanamge, Y., Hu, Y., and Shao, X. (2020). Application of wavelet transform in structural health monitoring. Earthquake Engineering and Engineering Vibrations, 19:515-532.

[71] Karami-Mohammadi, R., Mirtaheri, M., Salkhordeh, M., and Hariri-Ardebili, M. A. (2020). Vibration Anatomy and Damage Detection in Power Transmission Towers with Limited Sensors. Sensors, 20(6):1731.

[72] Kim, H. and Melhem, H. (2004). Damage detection of structures by wavelet analysis". Engineering Structures, 26(3):347-362.

[73] Kingma, D. and Ba, J. (2014). Adam: a method for stochastic optimization. In 3rd International Conference for Learning Representations. Diego, arXiv:1412.6980v9. 
[74] Kiranyaz, S., Avci, O., Abdeljaber, O., Ince, T., Gabbouj, M., and Inman, D. (2019). 1d convolutional neural networks and applications - a survey".

[75] Krizhevsky, A., Sutskever, I., and Hinton, G. (2012). Imagenet classification with deep convolutional neural networks. Communications of the ACM, 60(6):84-90.

[76] Kumar, R., Sumathi, P., Member, S., and Kumar, A. (2017). Synchrosqueezing transform-based frequency shifting detection for earthquake-damaged structures. IEEE Geoscience and Remote Sensing Letters, 14(8):1393-1397.

[77] Kumar, S., Wang, M., Abraham, D., Jahanshahi, M., Iseley, T., and Cheng, J. (2019). Deep learning-based automated detection of sewer defects in cctv videos. Journal of Computing in Civil EngineeringASCE, 34(1):04019047.

[78] Lecun, Y., Bottou, L., Bengio, Y., and Haffner, P. (1998). Gradient-based learning applied to document recognition. Proceedings of the IEEE, 86(11):2278-2324.

[79] Li, H., Yi, T., Ren, L., Li, D., and Huo, L. (2014). Reviews on innovations and applications in structural health monitoring for infrastructures. Structural Monitoring and Maintenance, 1(1):001-045.

[80] Li, S., Laima, S., and Li, H. (2017). Cluster analysis of winds and wind induced vibrations on a long-span bridge based on long-term field monitoring data. Engineering Structures, 138:245-259.

[81] Liang, X. (2019). Image-based post-disaster inspection of reinforced concrete bridge systems using deep learning with bayesian optimization. Computer-Aided Civil and Infrastructure Engineering, 34(5):415-430. 
[82] Liu, H. and Jiao, Y. (2011). Application of genetic algorithm-support vector machine for damage identification of bridge. International Journal of Computational Intelligence and Applications, 10(4):383-397.

[83] Liu, J., Zheng, J., Wei, X., Ren, W., and Laory, I. (2019). A combined method for instantaneous frequency identification in low-frequency structures. Engineering Structures, 194(05):370-383.

[84] Liu, T., Xu, H., Ragulskis, M., Cao, M., and Ostachowicz, W. (2020). A datadriven damage identification framework based on transmissibility function datasets and one-dimensional convolutional neural networks: Verification on a structural health monitoring benchmark structure. Sensors, 20(4):1-25.

[85] Liu, W., Chen, B., and Swartz, R. (2013). Investigation of time series representations and similarity measures for structural damage pattern recognition. The Scientific World Journal, 248349.

[86] Ljung, L. (1998). System identification. In A., P., J., U., P.W.J., R., and N.G, K., editors, Signal Analysis and Prediction. Applied and Numerical Harmonic Analysis. Birkhäuser, Boston, MA.

[87] Lo, C., Lynch, J., and Liu, M. (2013). Distributed reference-free fault detection method for autonomous wireless sensor networks. IEEE Sensor Journal, 13(5):2009-2019.

[88] Lu, J., Cheng, W., He, D., and Zi, Y. (2019). A novel underdetermined blind sources 
separation method with noise and unknown source number. Journal of Sound and Vibration, 457:67-91.

[89] Lynch, J. (2007). An overview of wireless structural health monitoring of civil structures. Philosophical Transactions of the Royal Society of London A: Mathematical, Physical and Engineering Sciences, 365(1851):345-372.

[90] Lynch, P. and Loh, J. (2006). A summary review of wireless sensors and sensor networks for structural health monitoring. The Shock and Vibration Digest, 38(2):91-128.

[91] Maeck, J. and G, R. D. (2003). Description of z24 benchmark. Mechanical Systems and Signal Processing, 17(1):127-131.

[92] Mahato, S. and Chakraborty, A. (2019). Sequential clustering of synchrosqueezed wavelet transform coefficients for efficient modal identification. Journal of Civil Structural Health Monitoring, 9:271-291.

[93] Mihalec, M., Slavi, J., and Bolte, M. (2016). Synchrosqueezed wavelet transform for damping identification. Mechanical Systems and Signal Processing, 80:324-334.

[94] Nakamura, M., F., M. S., Chassiakos, A., and K, C. T. (1998). Amethod for nonparametric damage detection through the use of neural networks. Earthquake Engineering and Structural Dynamics, 27:997-1010.

[95] Neild, S., McFadden, P., and Williams, M. (2003). A review of tf methods for structural vibration analysis. Engineering Structures, 25:713-728. 
[96] Newland, D. (1994). Wavelet analysis of vibration part i: Theory. Journal of Vibrations and Acoustics, 116:409-416.

[97] Nguyen, T., Chan, T., and Thambiratnam, D. (2014). Controlled monte carlo data generation for statistical damage identification employing mahalanobis squared distance. Structural Health Monitoring, 13(4):461-472.

[98] Ni, F., Zhang, J., and Noori, M. (2019). Deep learning for data anomaly detection and data compression of a long-span suspension bridge". Computer-Aided Civil and Infrastructure Engineering.

[99] Ni, F., Zhang, J., and Noori, M. (2020). Deep learning for data anomaly detection and data compression of a long-span suspension bridge. Computer-Aided Civil and Infrastructure Engineering, 35(7):685-700.

[100] Okayasu, M. and Yamasaki, T. (2019). Structural health monitoring system for remote inspection of material failure. Journal of Nondestructive Evaluation, 38(51).

[101] Park, J., Kim, J., Hong, D., Ho, D., and Yi, J. (2009). Sequential damage detection approaches for beams using time-modal features and artificial neural networks. Journal of Sound and Vibration, 323(1-2):451-474.

[102] Perez-Ramirez, C., Amezquita-Sanchez, J., Adeli, H., Valtierra-Rodriguez, M., Camarena-Martinez, D., and Romero-Troncoso, R. (2016). New methodology for modal parameters identification of smart, civil structures using ambient vibrations and synchrosqueezed wavelet transform. Engineering Applications of Artificial Intelligence, $48: 1-12$. 
[103] Piryonesi, S. and El-Diraby, T. (2019). Data analytics in asset management: costeffective prediction of the pavement condition index. Journal of Infrastructure Systems, $A S C E, 26(1)$.

[104] Pouyanfar, S., Sadiq, S., Yan, Y., Tian, H., Tao, Y., Reyes, M., and Iyengar, S. (2019). A survey on deep learning. ACM Computing Surveys, 51(5):1-36.

[105] Rehman, N. and Mandic, D. (2010). Multivariate empirical mode decomposition". Proceedings of The Royal Society A, 466:1291-1302.

[106] Roeck, D. G. and Teughels, A. (2004). Structural damage identification of the highway bridge z24 by fe model updating. Journal of Sound and Vibration, 278:589-610.

[107] Sadhu, A. (2015). An integrated multivariate empirical mode decomposition method towards modal identification of structures. Journal of Vibration and Control, 23(17):2727-2741.

[108] Sadhu, A., Narasimhan, S., and Antoni., J. (2017). A review of output- only structural mode identification literature employing blind source separation methods. $\mathrm{Me}$ chanical Systems and Signal Processing, 94:415-431.

[109] Sadhu, A., Sony, S., and Friesen, P. (2019). Evaluation of progressive damage in structures using tensor decomposition-based wavelet analysis.

[110] Salawu, O. (1997). Detection of structural damage through changes in frequency: a review. Engineering Structures, 19(9):718-723. 
[111] Salehi, H. and Burgueno, R. (2018). Emerging artificial intelligence methods in structural methods in structural engineering. Engineering Structures, 171:170-189.

[112] Sanchez-A, J., P., and Adeli, H. (2015). Synchrosqueezed wavelet transform- fractality model for locating, detecting and quantifying damage in smart highrise building structures. Smart Material and Structures, 24(6):065034.

[113] Santos, J., Cremona, C., Calado, L., Silveira, P., and Orcesi, A. (2016). On-line unsupervised detection of early damage. Structural Control and Health Monitoring, 23:1047-1069.

[114] Shang, Z., Sun, L., Xia, Y., and Zhang, W. (2020). Vibration-based damage detection for bridges by deep convolutional denoising autoencoder.

[115] Sharma, S. and Sen, S. (2020). One-dimensional convolutional neural network-based damage detection in structural joints. Journal of Civil Structural Health Monitoring, page 0123456789 .

[116] Simonyan, K. and Zisserman, A. (2014). Very Deep Convolutional Networks for Large-Scale Image Recognition. CoRR.

[117] Sohn, H. and Farrar, C. (2001). Damage diagnosis using time series analysis of vibration signals. Smart Materials and Structures, 10(3):446-451.

[118] Sony, S., Dunphy, K., Sadhu, A., and Capretz, M. (2020). A systematic review of convolutional neural network-based structural condition assessment techniques. Engineering Structures, 226:111347. 
[119] Sony, S., Dunphy, K., Sadhu, S., and Capretz, M. (2021). A systematic review of convolutional neural network-based structural condition assessment techniques. Engineering Structures, 226:111347.

[120] Sony, S., Laventure, S., and Sadhu, A. (2019). A literature review of next-generation smart sensing technology in structural health monitoring. Structural Control and Health Monitoring, 26(3).

[121] Sony, S. and Sadhu, A. (2020). Synchrosqueezing transform-based identification of time-varying structural systems using multi-sensor data. Journal of Sound and Vibration, 486:115576.

[122] Spencer, B., Ruiz-Sandoval, M., and Kurata, N. (2004). Smart sensing technology: opportunities and challenges. Structural Control and Health Monitoring, 11(4):349-368.

[123] Srinivasan, A. (1999). Note on the location of optimal classifiers in n-dimentional roc space. Technical Report PRG-TR-2-99,, Oxford University Computing Laboratory, England.

[124] Staszewski., W. and Robertson, A. (2006). Time-frequency and time-scale analyses for structural health monitoring. Philosophical Transactions of Royal Society, A365.

[125] Su, J., Xia, Y., and Weng, S. (2020). Review on field monitoring of high-rise structures. Structural Control and Health Monitoring, e2629.

[126] Su, W., Le, T., Huang, C., and Lin, P. (2017). Locating damaged storeys in a structure based on its identified modal parameters in cauchy wavelet domain. Applied Mathematical Modelling, 53:674-692. 
[127] Sun, H. and Büyüköztürk, O. (2018). The MIT Green Building benchmark problem for structural health monitoring of tall buildings. Structural Control and Health Monitoring, 25(3):e2115.

[128] Sun, H., Mordret, A., Prieto, G. A., Toksöz, M. N., and Büyüköztürk, O. (2017). Bayesian characterization of buildings using seismic interferometry on ambient vibrations. Mechanical Systems and Signal Processing, 85:468-486.

[129] Sun, L., Shang, Z., Xia, Y., Bhowmick, S., and Nagarajaiah, S. (2020). Review of bridge structural health monitoring aided by big data and artificial intelligence: From condition assessment to damage detection. Journal of Structural Engineering, 146(5).

[130] Szegedy, C., Liu, W., Jia, Y., Sermanet, P., Reed, S., Anguelov, D., Erhan, D., Vanhoucke, V., and Rabinovich, A. (2014). Going Deeper with Convolutions, arxiv:1409.4842.

[131] Tary, J., Herrera, R., and Van Der Baan, M. (2018). Analysis of time-varying signals using continuous wavelet and synchrosqueezed transforms. Philosophical Transactions of the Royal Society A: Mathematical, Physical and Engineering Sciences, 376(2126).

[132] Thakur, G. (2015). The synchrosqueezing transform for instantaneous spectral analysis. Excursions in Harmonic Analysis, Applied and Numerical Harmonic Analysis, 4:397-406.

[133] Ulriksen, M. and Damkilde, L. (2016). Structural damage localization by outlier analysis of signal-processed mode shapes-analytical and experimental validation. Mechanical Systems and Signal Processing, 68:1-14. 
[134] Wang, V. and Ong, K. (2015). Nonparamteric statistical formulations for structural health monitoring. Computers and Structures, 148:63-74.

[135] Wang, Z., Ren, W., and Liu, J. (2013). A synchrosqueezed wavelet transform enhanced by extended analytical mode decomposition method for dynamic signal reconstruction. Journal of Sound and Vibration, 332(22):6016-6028.

[136] Wang, Z.-C., Ren, W.-X., and Chen, G. (2018). Time-frequency analysis and applications in time-varying/nonlinear structural systems: A state-of-the-art review. Advances in Structural Engineering, 21(10):1562-1584.

[137] Wigner, E. (1932). On the quantum correction for thermodynamic equilibrium. Physical Review, 40:749.

[138] Wu, H.-T., Flandrin, P., and Daubechies, I. (2011). One or two frequencies? the synchrosqueezing answers". Advances in Adaptive Data Analysis, 03(01n02):29-39.

[139] Yang, J., Lei, P., Lin, S., and Huang, N. (2004). Hilbert-huang based approach for structural damage detection. Journal of Engineering Mechanics, 130:85-95.

[140] Yang, Y. and Nagarajaiah, S. (2013). Output-only modal identification with limited sensors using sparse component analysis. Journal of Sound and Vibration, $332(19): 4741-4765$.

[141] Yao, G., Tao, L., and Zhong, J. (2019). A review of convolutional-neural-networkbased action recognition". Pattern Recognition Letters, 118:14-22. 
[142] Yao, X., Yi, T., Qu, C., and Li, H. (2018). Blind modal identification using limited sensors through modified sparse component analysis by time-frequency method. Computer-Aided Civil and Infrastructure Engineering, 33(9):769-782.

[143] Ye, X., Jin, T., and Yun, C. (2019). A review on deep learning-based structural health monitoring of civil infrastructures. Smart Structures and Systems, 24(5):567-586.

[144] Yi, T.-H., Yao, X.-J., Qu, C.-X., and Li, H.-N. (2018). Clustering number determination for sparse component analysis during output-only modal identification. Journal of Engineering Mechanics, 145(1):04018122.

[145] Ying, Y., Garrett, J. H., Oppenheim, I. J., Soibelman, L., Harley, J. B., Shi, J., and Jin, Y. (2013). Toward Data-Driven Structural Health Monitoring: Application of Machine Learning and Signal Processing to Damage Detection. J. Comput. Civ. Eng., $27(6): 667-680$.

[146] Yu, K., Yang, K., and Bai, Y. (2014). Estimation of modal parameters using the sparse component analysis based underdetermined blind source separation. Mechanical Systems and Signal Processing, 45(2):302-316.

[147] Zhang, A., Wang, K., Li, B., Yang, E., Dai, X., Peng, Y., and Chen, C. (2017). Automated pixel-level pavement crack detection on 3d asphalt surfaces using a deeplearning network. Computer-Aided Civil and Infrastructure Engineering, 32(10):805-819.

[148] Zhang, L., Sun, L., and Shang, Z. (2016). Real-time reliability assessment based on acceleration monitoring for bridge. Science China Technology Sciences, 59(8):1294-1304. 
[149] Zhang, Y., Miyamori, Y., Mikami, S., and Saito, T. (2019a). Vibration-based structural state identification by a 1-dimensional convolutional neural network". ComputerAided Civil and Infrastructure Engineering, 34(9):822-839.

[150] Zhang, Y., Miyamori, Y., Mikami, S., and Saito, T. (2019b). Vibration-based structural state identification by a 1-dimensional convolutional neural network. ComputerAided Civil and Infrastructure Engineering, 34(9):822-839.

[151] Zhao, X., Li, S., Su, H., Zhou, L., and Loh, K. (2018). Image-based comprehensive maintenance and inspection method for bridges using deep learning. In ASME 2018 Conference on Smart Materials, Adaptive Structures and Intelligent Systems, SMASIS 2018, volume 2, page 1-7.

[152] Zhou, Q., Ning, Y., Zhou, Q., Luo, L., and Lei, J. (2012). Structural damage detection method based on random forests and data fusion. Structural Health Monitoring, 12(1):48-58.

[153] Zhou, S., Ma, Y., Liu, L., Kang, J., Ma, Z., and L., Y. (2018). Output-only modal parameter estimator of linear time-varying structural systems based on vector tar model and least squares support vector machine. Mechanical Systems and Signal Processing, $98: 722-755$.

[154] Zhou, Y., Maia, N., Sampaio, R., and Wahab, M. (2017). Structural damage detection using transmissibility together with hierarchical clustering analysis and similarity measure. Structural Health Monitoring, 16(6):711-731.

[155] Zhou, Y. and Sun, L. (2018). Effects of high winds on a long-span sea-crossing bridge 
based on structural health monitoring. Journal of Wind Engineering and Industrial Aerodynamics, 174:260-268. 